\title{
From discovery to precision measurements: electron identification, electron-like backgrounds, and measurement of the differential fiducial cross-sections of the Higgs boson in the four-lepton decay channel with the ATLAS detector
}

\author{
by \\ Kathleen Whalen \\ A thesis submitted to the \\ Faculty of Graduate Studies and Research \\ in partial fulfilment of the requirements \\ for the degree of \\ Doctor of Philosophy \\ Department of Physics \\ Carleton University \\ Ottawa-Carleton Institute for Physics \\ Ottawa, Ontario \\ January 2015
}

(C)Kathleen Whalen, 2015 


\section{Abstract}

The Higgs boson was proposed in 1964 as part of a solution to the problem of how certain subatomic particles acquire mass. Until its discovery at CERN's Large Hadron Collider in 2012, the Higgs boson was the last missing piece of the Standard Model of particle physics. This thesis studies the Higgs boson in the four-lepton decay channel: $H \rightarrow Z Z^{(*)} \rightarrow 4 \ell$, where $\ell=e, \mu$, using $\sim 25 \mathrm{fb}^{-1}$ of data recorded by the ATLAS detector at $\sqrt{s}=7 \mathrm{TeV}$ and $8 \mathrm{TeV}$ in 2011 and 2012, respectively.

Particular attention will be given to the role of electrons in the search for and study of the Higgs boson. An offline electron identification algorithm, the MultiLepton menu, was developed in order to efficiently distinguish true reconstructed electron objects from the hadronic background. It achieved an efficiency of $\sim 95 \%$ and excellent background rejection, and contributed to the discovery of the Higgs boson.

The reducible electron-like background in the $H \rightarrow Z Z^{(*)} \rightarrow 4 \ell$ channel has been studied, and a data-driven method for estimating the background contributions of the $Z+$ jets, $Z+b \bar{b}$, and $t \bar{t}$ processes was developed and implemented as a cross-check for the final analysis, published in 2014 .

Several precision measurements of the Higgs boson's properties have been performed in the $H \rightarrow Z Z^{(*)} \rightarrow 4 \ell$ channel, including the first measurements of the inclusive and differential fiducial cross-sections, using the method of binby-bin correction factors. The inclusive cross-section has been measured to be $\sigma_{\text {fid }}=2.11_{-0.47}^{+0.53}$ (stat.) \pm 0.8 (syst.) fb. Differential cross-sections have been measured for six observables; no significant deviations from theoretical Standard Model 
predictions have been observed. An alternative method for extracting the differential cross-sections, the Bayesian iterative unfolding method, has been studied and has been found to yield results consistent with the nominal results. This method shows particular promise for future precision measurements in the high-statistics regime, where resolution effects may degrade the performance of the method of correction factors. 


\section{Acknowledgements}

I'm still not entirely sure how I ended up here, and there were times when I was sure that I wouldn't. What I do know is that I couldn't have come this far without some very important people, to whom I owe my sincere gratitude and appreciation.

To my advisor, Prof. Manuella Vincter, for your patience and enthusiasm, and for your constant support throughout my studies.

To my many helpful colleagues in HSG2: Fabien Tarrade for putting up with my questions, Christos Anastopoulos for sharing your electron expertise, and Giacomo Artoni for your collaboration on the MultiLepton menu. To the "background girls," Eleonora Benhar Noccioli, Lydia Fayard, and Elodie Tiouchichine, for the endless comparisons and cross-checks. Special thanks to Ludovica Aperio Bella and Eleni Mountricha for all your advice and encouragement... and for the chocolate! To Sarah Heim, Gabi Pásztor, and Jon Stahlman for all your help with the cross-section studies.

To the Carletoneers: Graham Cree, David Di Valentino, Dag Gillberg, Louise Heelan, Jim Lacey, Tom McCarthy, and Rick Ueno for many years of helpful discussions and riddle-filled dinners, and for your willingness to take the results of my procrasti-baking off my hands.

To the ladies of the Casa de las Kimbambas, and everyone who made our house such a fun, welcoming place with good food, good music, and good friendship. To all my CERN friends all over the world.

To my family, for always supporting me, no matter what the endeavour, and for always believing in me, especially in times when I didn't believe in myself. 


\section{Contents}

Abstract

Acknowledgements

List of Tables $\quad$ ix

List of Figures $\quad$ xii

List of Abbreviations and Acronyms $\quad$ XV

1 Introduction 1

2 The Standard Model and the Higgs boson 5

2.1 The building blocks: quarks and leptons . . . . . . . . . . . . 6

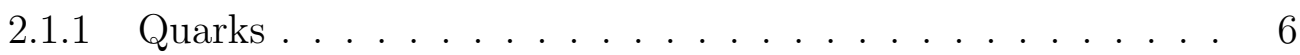

2.1 .2 Leptons . . . . . . . . . . . . . . . . . . . . . . . . . . . . . .

2.2 The fundamental interactions . . . . . . . . . . . . . . 8

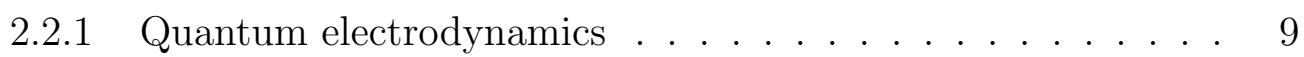

2.2.2 Quantum chromodynamics . . . . . . . . . . . . 10

2.2.3 The electroweak interaction and the Standard Model . . . . 12

2.3 Spontaneous symmetry breaking: the Higgs mechanism . . . . . . . 15

2.4 Previous experimental searches for the Higgs boson . . . . . . . . . 19

2.5 Higgs boson searches at the LHC . . . . . . . . . . . . . . . 20

2.5.1 Hadron collider physics . . . . . . . . . . . . . . . 20

2.5.2 Production modes . . . . . . . . . . . . . . . 21

2.5.3 Decay modes . . . . . . . . . . . . . . . 22

2.6 Conclusion . . . . . . . . . . . . . . 26

3 The LHC and the ATLAS Experiment $\quad 27$

3.1 The Large Hadron Collider . . . . . . . . . . . . . . . . . . . . . 27

3.2 The ATLAS Experiment . . . . . . . . . . . . . . . . . . . 32

3.2.1 Motivation and physics program . . . . . . . . . . 32

3.2.2 Overview of the ATLAS Experiment . . . . . . . . . . 33 
3.2.3 The ATLAS coordinate system . . . . . . . . . . . . . 35

3.2 .4 Inner detector . . . . . . . . . . . . . . . . . 36

3.2.4.1 Pixel detector . . . . . . . . . . . . . . . . . . . . . . . . . . . . 37

3.2.4.2 Semiconductor tracker . . . . . . . . . . . 38

3.2.4.3 Transition radiation tracker . . . . . . . . . . 38

3.2.5 Calorimeters . . . . . . . . . . . . . . . . 39

3.2.5.1 Electromagnetic calorimeter . . . . . . . . 40

3.2.5.2 Hadronic calorimeters . . . . . . . . . . . . . . 42

3.2 .6 Muon Spectrometer . . . . . . . . . . . . . . . . . . . . . . . . . . . . . . . . 43

3.2 .7 Trigger . . . . . . . . . . . . . . . . . . . 44

3.3 Physics object reconstruction _. . . . . . . . . . . . . . . . . . . . . . . . . . 46

3.3.1 Electron reconstruction . . . . . . . . . . . . . . . 46

3.3.2 Photon reconstruction . . . . . . . . . . . . . . . . . 49

3.3.3 Muon reconstruction ................. . . . 50

3.3 .4 Jet reconstruction . . . . . . . . . . . . . . . . . . . . . . 52

3.3.5 Missing transverse energy reconstruction . . . . . . . . . . 52

3.4 ATLAS Monte Carlo simulation . . . . . . . . . . . . . . . 53

3.5 Conclusion . . . . . . . . . . . . . . . . . 54

4 Offline electron identification in ATLAS $\mathbf{5 5}$

4.1 Discriminating variables used in offline electron identification . . . . 57

4.1 .1 Shower shape variables . . . . . . . . . . . . . . 58 58

4.1 .2 Track quality variables . . . . . . . . . . . . . . 66

4.1 .3 Track-cluster matching variables . . . . . . . . . . . . 70

4.2 The IsEM++ menu . . . . . . . . . . . . . . . . . . . 74

4.3 Offline electron identification in the $H \rightarrow Z Z^{(*)} \rightarrow 4 \ell$ search: the

MultiLepton menu . . . . . . . . . . . . . . 75

4.3.1 Motivation for the MultiLepton menu . . . . . . . . . 75

4.3.2 Implementation of the MultiLepton menu . . . . . . . . 76

4.3.2.1 Optimization of $\Delta \eta_{1} \ldots \ldots \ldots \ldots$. . . . . . 77

4.3.2.2 The use of $\Delta p / p$ for electron categorization and the optimization of $\Delta \phi_{\mathrm{R}} \ldots \ldots \ldots \ldots$

4.3.3 Performance of the MultiLepton menu . . . . . . . . . . . 80

4.3.4 Summary of the MultiLepton menu . . . . . . . . . . 87

4.4 The likelihood-based method for electron identification . . . . . . . 89

4.5 Conclusion . . . . . . . . . . . . . . . . . . . . . . 90

5 Event selection and background estimation 93

5.1 Signal samples . . . . . . . . . . . . . . . . . . . . . . . . . . . . . . . . . 94

5.2 Background samples . . . . . . . . . . . . . . . . . 96

5.3 Event selection . . . . . . . . . . . . . . . . . 96

5.3 .1 Data quality .................... 96 
5.3 .2 Trigger . . . . . . . . . . . . . . . . . 97

5.3 .3 Object selection . . . . . . . . . . . . . . . . . . . . . . 98

5.3.3.1 Electron selection . . . . . . . . . . . . . . . 98

5.3.3.2 Muon selection ............... . . 99

5.3 .3 .3 Jet selection . . . . . . . . . . . . . . . . 100

5.3.3.4 Removal of overlapping objects . . . . . . . . . . 101

5.3.4 Quadruplet formation and Higgs boson candidate selection . 101

5.3.5 Candidate event corrections . . . . . . . . . . . . . . . . 104

5.4 Background estimation . . . . . . . . . . . . . . . 105

5.4.1 Irreducible $Z Z^{(*)}$ background . . . . . . . . . . . . . . . . 105

5.4 .2 Reducible background . . . . . . . . . . . . . 107

5.4.2.1 Reducible $Z+\mu \mu$ background estimation . . . . . 109

5.4.2.2 Reducible $Z+$ ee background estimation . . . . . . 112

5.5 Conclusion . . . . . . . . . . . . . . . . . 115

6 A data-driven estimation of the reducible electron-like background in the $H \rightarrow Z Z^{(*)} \rightarrow 4 \ell$ channel: the Transfer Factor method 116

6.1 Categorization of the electron-like background . . . . . . . . . . . 118

6.1.1 Truth categories . . . . . . . . . . . . . . . 118

6.1.2 Reconstruction categories . . . . . . . . . . . . . . 119

6.2 The $Z+X X$ control region . . . . . . . . . . . . . . . . 120

6.3 Selection efficiency of electron-like background objects . . . . . . . . 122

6.3.1 The $Z+X$ control sample . . . . . . . . . . . . . . . . . . 122

6.3.2 Efficiencies for the reconstruction categories . . . . . . . . . 124

6.3.3 Efficiencies for the truth categories . . . . . . . . . . 126

6.3.4 Comparison of the $Z+X$ and $Z+X X$ samples and correction of the efficiencies . . . . . . . . . . . . . . . . 128

6.3.4.1 Selection of enriched control regions and derivation of efficiency scale factors . . . . . . . . . . . . . 131

6.4 Systematic uncertainties . . . . . . . . . . . . . . . . . . 134

6.5 Results of the Transfer Factor method using the $8 \mathrm{TeV}$ data . . . . 135

6.6 Modification of the Transfer Factor method for the $7 \mathrm{TeV}$ analysis .137

6.6.1 $Z+X X$ control regions in the $7 \mathrm{TeV}$ data . . . . . . . 137

$6.6 .2 Z+X$ efficiencies in the $7 \mathrm{TeV}$ data . . . . . . . . . . 140

6.6.3 Systematic uncertainties . . . . . . . . . . . . . . 146

6.6.4 Reducible background yield in the $7 \mathrm{TeV}$ data . . . . . . . . 146

6.7 Conclusion . . . . . . . . . . . . . . . . . . . . . . . 148

7 Results of the search for the Higgs boson in the $H \rightarrow Z Z^{(*)} \rightarrow 4 \ell$ $\begin{array}{lr}\text { channel } & 149\end{array}$

7.1 Results of the event selection . . . . . . . . . . . . . . . . . 150

7.2 Distributions of key observables . . . . . . . . . . . . . 151 
7.3 Measurement of the mass and signal strength . . . . . . . . . . 156

7.3.1 Systematic uncertainties . . . . . . . . . . . . 156

7.3.2 Results of the mass and signal strength measurements . . . . 158

7.4 Event categorization . . . . . . . . . . . . . . . 160

7.4.1 Systematic uncertainties . . . . . . . . . . . . . . . . . . . . . . . . . . . . . . . .

7.4.2 Results of the categorized analysis . . . . . . . . . . . . 162

7.5 Spin-parity measurement . . . . . . . . . . . . . . . 163

7.6 Conclusion . . . . . . . . . . . . . . . . . 166

8 Measurement of the inclusive and differential fiducial cross-sections in the $H \rightarrow Z Z^{(*)} \rightarrow 4 \ell$ channel $\quad 167$

8.1 Definition of the cross-section and observables of interest . . . . . 168

8.2 Definition of the fiducial region . . . . . . . . . . . . . . 171

8.3 Bin-by-bin unfolding . . . . . . . . . . . . . . . . 172

8.3.1 Correction factors . . . . . . . . . . . . . . . . 173

8.3.2 Study of the model-dependence of the correction factors . . 175

8.3 .3 Systematic uncertainties . . . . . . . . . . . . . . . 178

8.4 Results . . . . . . . . . . . . . . . . . . . 181

8.4.1 Inclusive fiducial cross-section . . . . . . . . . . . . . . 181

8.4.2 Differential fiducial cross-sections . . . . . . . . . . . . . 181

8.5 The Bayesian iterative unfolding method . . . . . . . . . . . . . . 185

8.5.1 Application of Bayes' theorem to the unfolding problem . . . 186

8.5.2 Bayesian iterative unfolding in the RooUnFolD framework 187

8.5.3 Systematic uncertainties . . . . . . . . . . . . . . 191

8.5.3.1 Effect of the reducible background shapes . . . . . 191

8.5.3.2 Effect of the number of iterations . . . . . . . . . . 192

8.6 Conclusion . . . . . . . . . . . . . . . . . . . . . . . 194

9 Summary and outlook $\quad 196$

$\begin{array}{ll}\text { A Personal contributions to ATLAS } & 201\end{array}$

$\begin{array}{ll}\text { B Selected presentations } & 203\end{array}$

C Monte Carlo samples 204

C.1 Signal samples. . . . . . . . . . . . . . . . . . . . . . . . . . . . . . . . . . . . . . . . .

C.1.1 $7 \mathrm{TeV}$ samples. . . . . . . . . . . . . . . . . . 204

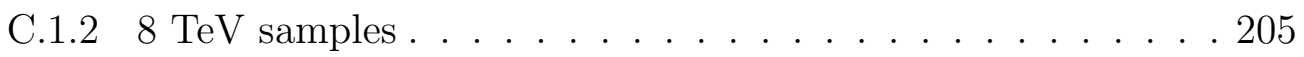

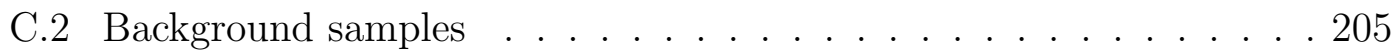

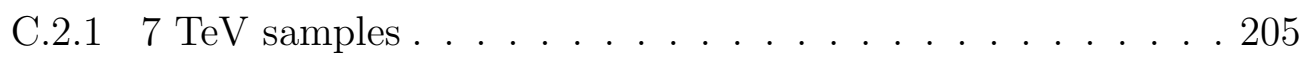

C.2.2 $8 \mathrm{TeV}$ samples................... . . 206

$\begin{array}{lr}\text { References } & 208\end{array}$ 


\section{List of Tables}

2.1 The three generations of quarks . . . . . . . . . . . . . 7

2.2 The three generations of leptons . . . . . . . . . . . . . 7

2.3 The four fundamental interactions of nature . . . . . . . . 8

4.1 Comparison of the Loose ++ , reoptimized Multilepton, and LIKELIHOOD menus . . . . . . . . . . . . . . . . 91

5.1 Higgs boson production cross-sections and decay branching ratios . 95

5.2 Trigger $p_{\mathrm{T}}$ thresholds used in the $7 \mathrm{TeV}$ and $8 \mathrm{TeV}$ analyses . . . . 97

5.3 Selection criteria for physics objects . . . . . . . . . . . . . 98

5.4 Muon track quality requirements for the $7 \mathrm{TeV}$ and $8 \mathrm{TeV}$ analyses 100

5.5 Mass points used to interpolate the minimum threshold for the $m_{34}$ cut . . . . . . . . . . . . . . . . . . . 102

5.6 Estimated reducible $Z+\mu \mu$ background yield in the $7 \mathrm{TeV}$ and $8 \mathrm{TeV}$ data . . . . . . . . . . . . . . . . . . . . . 112

5.7 Reducible $Z+e$ background yield in the $7 \mathrm{TeV}$ and $8 \mathrm{TeV}$ data determined using the $3 \ell+X$ method . . . . . . . . . . . 115

6.1 Electron reconstruction category selection criteria . . . . . . . . . 120

6.2 Observed and expected yields in the $Z+X X$ control region in the $8 \mathrm{TeV}$ data and Monte Carlo . . . . . . . . . . . . . . . 122

6.3 Composition of the $Z+X X$ control region in terms of reconstruction categories, as observed in the $8 \mathrm{TeV}$ data . . . . . . . . . . . . 122

$6.4 Z+X$ event yields in the $8 \mathrm{TeV}$ data . . . . . . . . . . . . 123

6.5 Reconstruction-categorized efficiencies for background objects in the $8 \mathrm{TeV}$ data and Monte Carlo . . . . . . . . . . . . . 126

6.6 Truth efficiencies for background objects in the $8 \mathrm{TeV}$ data and Monte Carlo . . . . . . . . . . . . . . . . . . . 126

6.7 Composition of the $Z+X$ sample and $Z+X X$ control region using the $8 \mathrm{TeV}$ Monte Carlo . . . . . . . . . . . . . . . . . . . . . . . . 129

6.8 Scale factors used to correct Monte Carlo truth efficiencies for the $\gamma$ and $f$ components in the $8 \mathrm{TeV}$ reducible background analysis. . 134

6.9 The reducible background estimate for the $8 \mathrm{TeV}$ data, determined using the Transfer Factor method . . . . . . . . . . . . . . . 136 
6.10 Observed and expected yields in the $Z+X X$ control region CR5 in the $7 \mathrm{TeV}$ data and Monte Carlo . . . . . . . . . . . . . . . . 138

6.11 Observed and expected yields in the $Z+X X$ control region CR5a in the $7 \mathrm{TeV}$ data and Monte Carlo . . . . . . . . . . . . . 139

6.12 Composition of the $Z+X X$ control region CR5 in terms of reconstruction categories, as observed in the $7 \mathrm{TeV}$ data . . . . . . . 139

6.13 Composition of the $Z+X X$ control region CR5a in terms of reconstruction categories, as observed in the $7 \mathrm{TeV}$ data . . . . . . . 139

6.14 Reconstruction-categorized efficiencies for background objects in the $7 \mathrm{TeV}$ data and Monte Carlo . . . . . . . . . . . . . 140

6.15 Truth efficiencies for background objects in the $7 \mathrm{TeV}$ data and Monte Carlo . . . . . . . . . . . . . . . . . . . . . . . . . . 140

6.16 Composition of the $Z+X$ sample and $Z+X X$ control region using the $7 \mathrm{TeV}$ Monte Carlo . . . . . . . . . . . . . . . . . . . . . . . . . 144

6.17 The reducible background estimate for the $7 \mathrm{TeV}$ data, determined using the Transfer Factor method with CR5 . . . . . . . . . . . 147

6.18 The reducible background estimate for the $7 \mathrm{TeV}$ data, determined using the Transfer Factor method with CR5a . . . . . . . . . . . 147

6.19 Summary of the estimated reducible background yields in the $7 \mathrm{TeV}$ and $8 \mathrm{TeV}$ data determined using the Transfer Factor method . . . 148

7.1 Total observed events in data for the $7 \mathrm{TeV}$ and $8 \mathrm{TeV}$ analyses . . 150

7.2 Expected and observed signal and background yield in the mass window $110-140 \mathrm{GeV}$. . . . . . . . . . . . . . . 150

7.3 Sources of systematic uncertainty on the signal strength measurement 158

7.4 Expected and observed signal and background yields for the categorized analysis . . . . . . . . . . . . . . . . 162

8.1 Binning for the six observables used for the differential cross-section measurement . . . . . . . . . . . . . . . 170

8.2 Definition of the fiducial region . . . . . . . . . . . . . 172

8.3 Inclusive correction factors for each Higgs boson production mode . 175

8.4 Correction factors for the inclusive and differential cross-section measurements . . . . . . . . . . . . . 176

8.5 Summary of the systematic uncertainties on the differential crosssection measurement . . . . . . . . . . . . . . . . . 180

8.6 Comparison of measured differential cross-sections with theoretical calculations . . . . . . . . . . . . . . . . 185

C.1 List of $7 \mathrm{TeV}$ signal Monte Carlo samples . . . . . . . . . . . . . . . 204

C.2 List of $8 \mathrm{TeV}$ signal Monte Carlo samples . . . . . . . . . . . . . . . 205

C.3 List of $7 \mathrm{TeV}$ reducible background Monte Carlo samples . . . . . . 206

C.4 List of $7 \mathrm{TeV}$ irreducible background Monte Carlo samples . . . . . 206 
C.5 List of $8 \mathrm{TeV}$ reducible background Monte Carlo samples . . . . . . 206

C.6 List of $8 \mathrm{TeV}$ irreducible background Monte Carlo samples . . . . . 207 


\section{List of Figures}

2.1 Schematic of a proton-proton collision at the LHC . . . . . . . . . 21

2.2 Higgs boson production mechanisms at the LHC . . . . . . . . . . . 23

2.3 Higgs boson production cross-sections as a function of the Higgs boson mass . . . . . . . . . . . . . . . . . . . . . 24

2.4 Higgs boson cross-sections, multiplied by the branching ratio for its decay modes, as a function of the Higgs boson mass . . . . . . . . 25

3.1 The CERN accelerator complex . . . . . . . . . . . . . . 28

3.2 Peak instantaneous luminosity delivered to ATLAS by the LHC . . 31

3.3 Mean number of interactions per bunch crossing . . . . . . . . . . 31

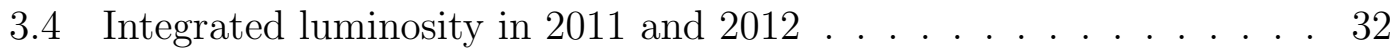

3.5 The ATLAS detector . . . . . . . . . . . . . . . . . 34

3.6 An example of a $Z \rightarrow \mu \mu$ event featuring high pileup in ATLAS . . 35

3.7 Schematic of the ATLAS coordinate system . . . . . . . . . . . 36

3.8 The ATLAS inner detector . . . . . . . . . . . . . . . . . . . . . . . . . . 37

3.9 The ATLAS pixel detector, SCT, and TRT . . . . . . . . . . . 39

3.10 Amount of material in front of the cryostat . . . . . . . . . . . . . 40

3.11 The ATLAS calorimeter system . . . . . . . . . . . . . . . . . . . . 41

3.12 An electromagnetic calorimeter module . . . . . . . . . . . . . . 42

3.13 The ATLAS muon spectrometer . . . . . . . . . . . . . . . . 44

3.14 Physics object reconstruction in ATLAS . . . . . . . . . . . . 47

4.1 Composition of reconstructed electron objects . . . . . . . . . . 56

$4.2 E_{\text {ratio }}$ distributions of reconstructed electron candidates . . . . . . . 60

$4.3 w_{\text {s, tot }}$ distributions of reconstructed electron candidates . . . . . . . 61

$4.4 R_{\eta}$ distributions of reconstructed electron candidates . . . . . . . 63

$4.5 w_{\eta 2}$ distributions of reconstructed electron candidates . . . . . . . . 64

$4.6 f_{3}$ distributions of reconstructed electron candidates . . . . . . . . . 65

$4.7 R_{\text {had }}$ distributions of reconstructed electron candidates . . . . . . . 67

$4.8 N_{\text {pix }}$ and $N_{\mathrm{Si}}$ distributions of reconstructed electron candidates . . . 68

$4.9 R_{\mathrm{TRT}}$ distributions of reconstructed electron candidates . . . . . . . 69

$4.10 \Delta p / p$ distributions of reconstructed electron candidates . . . . . . . 71

$4.11 \Delta \eta_{1}$ distributions of reconstructed electron candidates . . . . . . . . 72 
$4.12 \Delta \phi_{\mathrm{R}}$ distributions of reconstructed electron candidates . . . . . . . 73

4.13 Reoptimized cuts on $\Delta \eta_{1}$. . . . . . . . . . . . . . . . . . 78

4.14 The use of $\Delta p / p$ to determine thresholds for bremsstrahlung emission 80

4.15 Reoptimized cuts on $\Delta \phi_{\mathrm{R}}$, using bremsstrahlung categories. . . . . . 81

4.16 Electron efficiency of the MultiLepton menu, compared to the Looset+ menu . . . . . . . . . . . . . . . 82

4.17 Hadron rejection of the MultiLepton menu, compared to the Looset+ menu . . . . . . . . . . . . . . . 83

4.18 Conversion rejection of the MultiLepton menu, compared to the Looset+ menu . . . . . . . . . . . . . . . . 84

$4.19 N_{\mathrm{BL}}$ distributions of reconstructed electron candidates . . . . . . 85

4.20 Electron efficiency of the reoptimized MultiLepton menu, compared to the original MultiLepton menu . . . . . . . . . 86

4.21 Hadron rejection of the reoptimized MultiLepton menu, compared to the original MultiLepton menu . . . . . . . . . . . 87

4.22 Conversion rejection of the reoptimized MultiLePton menu, compared to the original MultiLePton menu . . . . . . . . . . 88

5.1 Effect of FSR recovery on the $Z$ boson invariant mass distribution .104

5.2 Comparison of the $H \rightarrow Z Z^{(*)} \rightarrow 4 \ell$ signal and irreducible $Z Z^{(*)} \rightarrow$ $4 \ell$ background final states . . . . . . . . . . . . . 106

5.3 Example BDT output scores for simulated signal and $Z Z^{(*)}$ events. 106

5.4 Example Feynman diagrams for the main sources of reducible background to the $H \rightarrow Z Z^{(*)} \rightarrow 4 \ell$ signal . . . . . . . . . . . . . 108

5.5 Invariant mass distributions in the $Z+\mu \mu$ background control region 109

5.6 Observed leading dimuon invariant mass distributions and fit results in the $Z+\mu \mu$ control regions . . . . . . . . . . . . . . 111

5.7 Invariant mass distributions in the $Z+e e$ background control region 113

5.8 Observed distributions and fit results in the $Z+e$ control regions . 114

6.1 Reconstruction-categorized efficiencies for background objects in the $8 \mathrm{TeV}$ data and Monte Carlo . . . . . . . . . . . . . . 125

6.2 Truth efficiencies for true isolated electrons $(e)$ in the $8 \mathrm{TeV}$ Monte Carlo . . . . . . . . . . . . . . . . . . 127

6.3 Truth efficiencies for electrons from heavy quark decays $(q)$ in the $8 \mathrm{TeV}$ Monte Carlo . . . . . . . . . . . . . . . 127

6.4 Truth efficiencies for photon conversions and FSR $(\gamma)$ in the $8 \mathrm{TeV}$ Monte Carlo . . . . . . . . . . . . . . . . . . . . . . . . . . 128

6.5 Truth efficiencies for fakes $(f)$ in the $8 \mathrm{TeV}$ Monte Carlo . . . . . 128

6.6 Corrected $Z+X$ efficiencies . . . . . . . . . . . . . . . . . . . 133

6.7 Reconstruction-categorized efficiencies for background objects in the $7 \mathrm{TeV}$ data and Monte Carlo . . . . . . . . . . . . . . . . 141 
6.8 Truth efficiencies for true isolated electrons $(e)$ in the $7 \mathrm{TeV}$ Monte Carlo . . . . . . . . . . . . . . . . . . . . . . 142

6.9 Truth efficiencies for electrons from heavy quark decays $(q)$ in the $7 \mathrm{TeV}$ Monte Carlo . . . . . . . . . . . . . . . . . . . . . 142

6.10 Truth efficiencies for photon conversions and FSR $(\gamma)$ in the $7 \mathrm{TeV}$ Monte Carlo . . . . . . . . . . . . . . . . . . . . . . . . 143

6.11 Truth efficiencies for fakes $(f)$ in the $7 \mathrm{TeV}$ Monte Carlo . . . . . 143

6.12 Corrected $Z+X$ efficiencies for the $7 \mathrm{TeV}$ analysis . . . . . . . . 145

$7.1 \quad H \rightarrow Z Z^{*} \rightarrow 2 e 2 \mu$ candidate event display . . . . . . . . . . 151

7.2 Four-lepton invariant mass $\left(m_{4 \ell}\right)$ distributions . . . . . . . . . . . 152

7.3 Four-lepton invariant mass $\left(m_{4 \ell}\right)$ distributions for the low mass range153

7.4 Leading dilepton invariant mass $\left(m_{12}\right)$ distributions . . . . . . . . 153

7.5 Subleading dilepton invariant mass $\left(m_{34}\right)$ distributions . . . . . . 154

7.6 Four-lepton transverse momentum $\left(p_{\mathrm{T}, 4 \ell}\right)$ distributions . . . . . . . 154

7.7 Four-lepton pseudorapidity $\left(\eta_{4 \ell}\right)$ distributions . . . . . . . . . . 155

7.8 BDT discriminant distributions . . . . . . . . . . . . . . . . 155

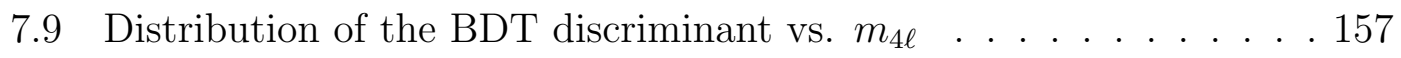

7.10 The observed local $p_{0}$ and signal strength as a function of $m_{H}$. . 159

7.11 Schematic of the event categorization procedure . . . . . . . . . 160

7.12 Production and decay angles in the $H \rightarrow Z Z^{(*)} \rightarrow 4 \ell$ decay . . . . . 164

7.13 BDT discriminant for the $J^{P}=0^{+}$and $0^{-}$hypotheses . . . . . . . 165

7.14 MELA discriminant for the $J^{P}=0^{+}$and $1^{+}$hypotheses . . . . . . 166

8.1 Schematic of truth-level lepton kinematics and photon emission . . 175

8.2 Comparison of correction factors for various spin/parity hypotheses 177

8.3 Reducible background shape variations used to evaluate the systematic uncertainty . . . . . . . . . . . . . . . . . 179

8.4 Observed yields for the six observables used for the differential crosssection measurements . . . . . . . . . . . . . . 182

8.5 Differential cross sections for the Higgs boson in the $H \rightarrow Z Z^{(*)} \rightarrow$ $4 \ell$ channel. . . . . . . . . . . . . . . . . . . . . . . . . 183

8.6 Response matrices for the six observables of interest. . . . . . . . 189

8.7 Comparison of unfolded differential cross-sections determined using the Bayesian unfolding method. . . . . . . . . . . . . . . . 190

8.8 Deviation from the nominal result due to varying the reducible background shapes . . . . . . . . . . . . . . . . 193 


\title{
List of Abbreviations and Acronyms
}

\author{
ALICE A Large Ion Collider Experiment \\ ATLAS A Toroidal LHC Apparatus \\ BDT Boosted decision tree \\ BR Branching ratio \\ CaloTag Calorimeter-tagged muon \\ CB Combined muon \\ CERN European Organization for Nuclear Research \\ CMS Compact Muon Solenoid \\ CP Charge conjugation parity symmetry \\ CR Control region \\ CR5 Control region with at least one subleading lepton failing a cut \\ CR5a Control region with both subleading leptons failing cuts \\ CSC Cathode strip chambers \\ $\boldsymbol{d}_{\mathbf{0}} \quad$ Transverse impact parameter \\ $\boldsymbol{E} \quad$ Electron-like background object \\ $\boldsymbol{E}_{\mathrm{T}} \quad$ Transverse energy \\ $\boldsymbol{E}_{\mathrm{T}}^{\mathrm{miss}} \quad$ Missing transverse energy \\ EF Event filter \\ EM Electromagnetic
}


EW Electroweak

$\boldsymbol{F} \quad$ Fake-like background object

fb Femtobarn

FCal Forward calorimeter

FSR Final state radiation

$\mathrm{GeV} \quad$ Gigaelectronvolt

ggF Gluon-gluon fusion

GSF Gaussian sum filter

ID Inner detector

LEP Large Electron-Positron Collider

LHC Large Hadron Collider

Linac2 Linear accelerator

MC Monte Carlo simulation

MDT Monitored drift tubes

MELA Matrix element-based likelihood analysis

MS Muon spectrometer

LO Leading order

NLO Next-to-leading order

NNLO Next-to-next-to leading order

PDF Parton distribution function

pdf Probability density function

PS Proton Synchrotron

PSB Proton Synchrotron Booster

$\boldsymbol{p}_{\mathrm{T}} \quad$ Transverse momentum

QCD Quantum chromodynamics

QED Quantum electrodynamics 
RPC Resistive plate chambers

SA Stand-alone muon

SCT Semiconductor tracker

SF Scale factor

SM Standard Model

SPS Super Proton Synchrotron

SR Signal region

ST Segment-tagged muon

TDAQ Trigger and data acquisition

TeV Teraelectronvolt

TF Transfer factor

TGC Thin gap chambers

TRT Transition radiation tracker

$\boldsymbol{t} \overline{\boldsymbol{t}} \mathbf{H} \quad$ Higgs production in association with a top-antitop quark pair

VBF Vector boson fusion

VH Higgs production in association with a vector boson

vev Vacuum expectation value

$\boldsymbol{X} \quad$ Reconstructed electron object satisfying relaxed cuts 
MY TEACHER ALWAYS TOLD ME THAT IF I APPLIED MYSELF, I COUD BECOME THE NEXT MARIE CORIE.
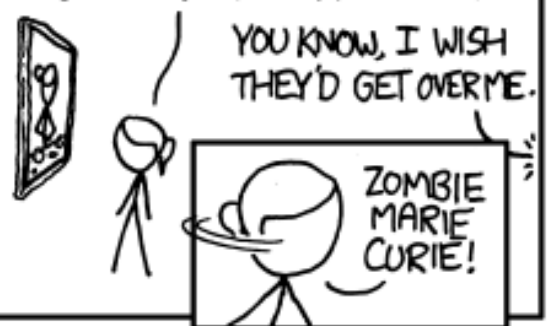

NOT THAT I DON'T DESERVE $\Pi$. THESE TWO NOBESS AINT DECORATIVE. BUT I MAKE A SORRY ROLE MOCEI IF GIRS JUST SEE ME OVER AND OVER AS THE ONE TOKEN LADY SCLENTIST.
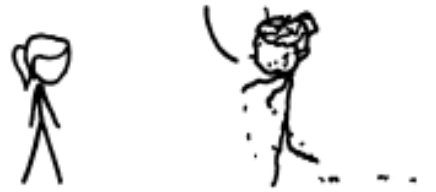

LISE MEITNER FIGURED OUT THAT NUCLEAR FISSION WAS HAPPENING, WHILE HER COLLEAQUE OTTO WAS STARING BLANKLY AT THEIR DATA IN CONFUSION, AND PROVED ENRICO FERMI WRONG IN THE PROCESS. ENRICO AND OTTO BOTH GOT NOBEL PRIZES. LSE GOT A NATIONAL WOMEN'S PRESS CLUB AWARD.

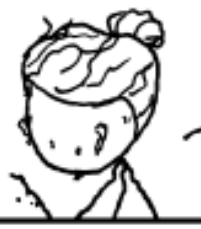
) THEY FINALLY NAMED AN ELEMENT AFTER HER, BUT NOTUNTIL 60 YEARS LATER.

EMMY NOETHER FOUGHT PAST HERVICTORIAN-ERA FINISHING-SCHOOL UPBRINGING PURSUED MATHEMATICS BYAUDTING CLASSES, AND, AFTER FINAULY GETING A PH.D, WAS PERMITTED TO TEACH ONLY AS AN UNPAID LECTURER (OFTEN UNDER MALE COLLEAQUES' NAMES). WAS SHE AS GOOD AS THEM?

$\mathrm{OH}$.

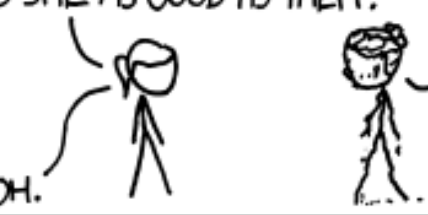
$\int_{1}$ SHE REVOLUTIONIZED ABSTRACT ALCEBRA, FILLD GAPS IN RELATIVTTY, AND FOUND WHAT SOME CALL THE MOST BEAUTIFUL, DEEPEST RESUIT IN THEORETCALPHYSKS.

BUT YOU DON'T BECOME GREAT BY TRYNG TO BE GREAT. YOU BECOME GREAT BY WANTNG TO DO SOMETHING, AND THEN DOING IT SO HARD THAT YOU BECOME GREAT IN THE PROCESS.

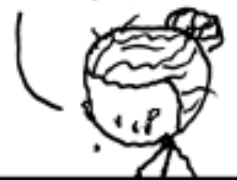

SO DONT TRY TO BE THE NEXT ME, NOETHER, OR MEITNER. JUST REMEMBER THAT IF YOU WANT TO DO THIS STUFF, YOU'RE NOT ALONE.

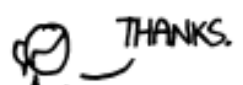
ALSO, AVOID RADIUM' TURNS OUTITKIUSYOU. I'L TRY. 


\section{Introduction}

The best that most of us can hope to achieve in physics is simply to misunderstand at a deeper level.

— Wolfgang Pauli

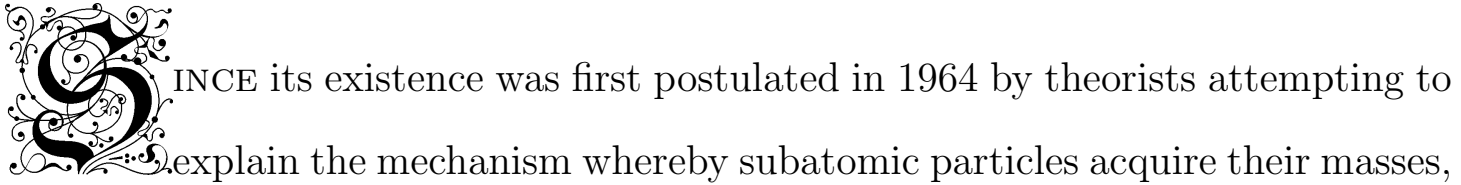
the search for the Higgs boson has proven to be a formidable challenge for physicists seeking to understand the fundamental interactions of the matter that makes up our Universe. The discovery of the Higgs boson at CERN's Large Hadron Collider in 2012 [2] was the culmination of a half-century-long international effort by thousands of people working to develop new detector technologies and understand precisely the signatures of Higgs boson decays in the detector, in order to accurately measure a clean signal and perform precision measurements of its properties.

This thesis attempts to provide some insight into the evolution of the analysis - from the theoretical justification for the existence of the Higgs boson, to the experimental search, to the discovery, to the first precision measurements - 
of one of the possible Higgs boson signatures, the decay to two $Z$ bosons, which subsequently decay to the four-lepton final state: $H \rightarrow Z Z^{(*)} \rightarrow 4 \ell$.

Chapter 2 presents a brief theoretical outline of the Standard Model, which encompasses the subatomic particles that make up the visible matter of our Universe, as well as three of the four fundamental forces governing their interactions. Particular attention is given to the theory of the electroweak interaction and the role of the Higgs mechanism, which gives mass to the particles mediating the interaction and results in the existence of a massive scalar particle: the Higgs boson.

After the theoretical foundations have been laid, the focus of this thesis will shift to the experimental search for the Higgs boson. Chapter 3 describes the Large Hadron Collider and one of its two general-purpose experiments: ATLAS, which was designed with the discovery of the Higgs boson at the top of its list of scientific objectives. The technology used to detect, reconstruct, and measure the properties of a broad range of subatomic particles will be summarized.

Chapter 4 concentrates on the identification of a particular particle: the electron, which is one of the possible final-state particles in the $H \rightarrow Z Z^{(*)} \rightarrow 4 \ell$ decay channel. Since Higgs boson production is a rare process, efficient identification of electrons and rejection of background is crucial in order to achieve the best possible acceptance of the cleanest possible signal. In order to maximize the experiment's discovery potential, the author of this thesis has developed an algorithm [3] to distinguish true electrons from the many imposters lurking in the background with high degrees of signal efficiency and background rejection. The development of this algorithm, which was implemented for the discovery of the Higgs boson, will be discussed in detail. 
Chapter 5 presents the analysis requirements of the search for the Higgs boson in the four-lepton decay channel. The criteria for selecting the leptons making up the final state, and for ensuring that those leptons were most likely the end products of a decaying Higgs boson, will be outlined.

With the discovery of what appeared to be a Higgs-like boson in 2012, the goal of the experiment immediately became the collection of even larger amounts of even cleaner data in order to be able to determine whether this new particle was, in fact, the Standard Model Higgs boson. One cannot collect a clean signal without first understanding the background; i.e. other Standard Model physics processes which produce similar (or even identical) signatures in the detector as the desired signal process. These processes may sometimes be difficult to accurately reproduce using simulations, thus necessitating the use of data-driven methods. Chapter 6 illustrates the development of one such method by the author of this thesis for understanding the composition of the electron-like background in the $H \rightarrow Z Z^{(*)} \rightarrow 4 \ell$ channel and estimating its contribution to the measured signal.

Chapter 7 presents the results of the event selection and background estimation discussed in the preceding chapters. A broad survey of the most up-to-date analysis of the dataset spanning the years 2011-2012 will be given, featuring measurements of the Higgs boson's mass [4], as well as several other properties which provide insight into whether it conforms to the predictions of the Standard Model, including its spin, parity [5], and production modes [6]. This chapter is intended to provide a survey for general interest; the author's most significant contribution to these results is the reducible background estimation described in the preceding chapter.

Some final precision measurements will be featured in more detail in Chapter 8: the first measurements of the Higgs boson's inclusive and differential fiducial 
production cross-sections in the four-lepton decay channel [7]. After presenting the official analysis procedure and results, an alternative method, which relies on Bayes' theorem of probability to extrapolate the true cross-section from the measured signal, will be discussed in detail. The author has found that this method, which accurately reproduces the results of the baseline analysis, offers additional advantages and appears to provide a promising outlook for future measurements when the LHC resumes operations in 2015.

Finally, Chapter 9 will summarize the work presented in the preceding chapters, spanning the initial search for the Higgs boson at the LHC, to its discovery and the study of its properties, and attempt to provide an outlook for future measurements during Run II at the LHC.

A summary of the author's personal contributions to ATLAS, in terms of physics analysis and detector-oriented service work, is given in Appendix A; Appendix B lists the major workshops and conferences at which aspects of this work have been presented. A list of the Monte Carlo samples used for this analysis is given in Appendix C. 


\title{
2. The Standard Model and the Higgs bo- SOn
}

If you wish to make an apple pie from scratch, you must first invent the universe.

- Carl Sagan, Cosmos

\begin{abstract}
(5.2. Sour Universe, as well as its interactions via the fundamental forces of nature. Many decades of experimental and theoretical research have resulted in the development of the Standard Model: a mathematical framework that describes the elementary particles and three of the forces that govern their interactions in terms of their underlying symmetries.
\end{abstract}

The groundwork for the Standard Model was laid out in the 1960s in order to explain the vast array of new subatomic particles being discovered due to the technological breakthroughs of that era. Since then, the Standard Model has proved to be one of the most successful theories to date; it successfully explained existing phenomena with high degrees of precision, as well as providing constraints on the properties of new particles, such as the top quark [8,9], which would not be discovered until decades later. Its most significant - and most difficult to 
confirm - prediction was the existence of the Higgs boson, which is a consequence of the Higgs mechanism: the mechanism whereby subatomic particles acquire their masses. The Higgs boson was finally discovered in 2012 [2]; this triumph of the Standard Model resulted in a Nobel Prize in 2013 for two of the theorists who predicted the existence of the boson and the associated mechanism.

This chapter will briefly summarize the Standard Model's constituent particles and interactions, including the Higgs boson, concluding with a discussion of the Higgs boson's decay via the four-lepton channel, which is the subject of this thesis.

\subsection{The building blocks: quarks and leptons}

Matter is made up of fermions: subatomic particles with half-integer spins. The fermions can be further subdivided into two categories: the leptons, which have integer electric charges (in units of the electron charge: $e=1.602 \times 10^{-19} \mathrm{C}$ ), and the quarks, which have fractional electric charges. Each fermion $f$ also has a corresponding antifermion, denoted by $\bar{f}$, which has opposite charge and other quantum numbers.

\subsubsection{Quarks}

The quarks are organized in three generations, each having one quark with charge $+2 / 3$ and one with charge $-1 / 3$ : up $(u)$ and down $(d)$, charm $(c)$ and strange $(s)$, and top $(t)$ and bottom $(b)$. Quarks also carry another form of charge, known as "colour" charge, which may be whimsically represented as red, blue, or green. Due to a particular property known as quark confinement, free quarks are not observed 
directly; rather, they exist in bound states of two or three quarks, known as hadrons, which are colourless and have integer charge. The most familiar hadrons are the proton $(u u d)$ and the neutron $(u d d)$, which are found in atomic nuclei. The quarks' properties are summarized in Table 2.1.

\begin{tabular}{lccc}
\hline \hline Generation & Quark & Charge & Mass $(\mathrm{MeV})$ \\
\hline First & $u$ & $+2 / 3$ & $2.3_{-0.5}^{+0.7}$ \\
Second & $d$ & $-1 / 3$ & $4.8_{-0.3}^{+0.5}$ \\
& $c$ & $+2 / 3$ & $(1.275 \pm 0.025) \times 10^{3}$ \\
\multirow{2}{*}{ Third } & $s$ & $-1 / 3$ & $(95 \pm 5)$ \\
& $t$ & $+2 / 3$ & $(173.21 \pm 0.51 \pm 0.71) \times 10^{3}$ \\
& $b$ & $-1 / 3$ & $(4.18 \pm 0.03) \times 10^{3}$ \\
\hline \hline
\end{tabular}

TABLE 2.1: The three generations of quarks, and their properties [10].

\subsubsection{Leptons}

Leptons are also organized in three generations, each consisting of a massive particle with charge -1 and an almost massless neutrino having 0 charge. The three generations are as follows: the electron $(e)$ and the electron neutrino $\left(\nu_{e}\right)$, the muon $(\mu)$ and the muon neutrino $\left(\nu_{\mu}\right)$, and the tau $(\tau)$ and the tau neutrino $\left(\nu_{\tau}\right)$. The properties of the leptons are summarized in Table 2.2.

\begin{tabular}{lccc}
\hline \hline Generation & Lepton & Charge & Mass $(\mathrm{MeV})$ \\
\hline First & $e$ & -1 & $0.510990928 \pm 0.000000011$ \\
& $\nu_{e}$ & 0 & $<2 \times 10^{-6}$ \\
Second & $\mu$ & -1 & $105.6583715 \pm 0.0000035$ \\
& $\nu_{\mu}$ & 0 & $<2 \times 10^{-6}$ \\
Third & $\tau$ & -1 & $1776.82 \pm 0.16$ \\
& $\nu_{\tau}$ & 0 & $<2 \times 10^{-6}$ \\
\hline \hline
\end{tabular}

TABLE 2.2: The three generations of leptons, and their properties [10]. 


\subsection{The fundamental interactions}

The interactions of elementary particles can be described in terms of four fundamental forces: the strong force, which binds quarks together to form hadrons; electromagnetism, which unifies electricity and magnetism; the weak force, which is responsible for weak nuclear decays; and gravity. The Standard Model encompasses the first three interactions; the effects of gravity are negligible at the subatomic level, and a viable quantum theory of gravity does not yet exist. Each fundamental interaction is mediated by particles known as gauge bosons, which have integer spin. The fundamental interactions, their mediators, and their relative strengths are listed in Table 2.3.

\begin{tabular}{lcc}
\hline \hline Interaction & Mediator & Coupling constant \\
\hline Strong & gluon & 1 \\
Electromagnetic & photon & $1 / 137$ \\
Weak & $W \& Z$ bosons & $10^{-6}$ \\
Gravitational & graviton (hypothetical) & $10^{-39}$ \\
\hline \hline
\end{tabular}

TABLE 2.3: The four fundamental interactions of nature, with their mediators and relative strengths in terms of their coupling constants.

The Standard Model is a gauge theory, in which particles are treated as excitations of fields. The Lagrangian $(\mathcal{L})$, which describes the dynamics of the system, is required to be invariant under a group of local transformations; i.e. it must be gauge invariant. This idea stems from Noether's theorem [11], which states that invariance of a system under a transformation, corresponding to a particular symmetry of nature, implies the existence of a conservation law for a physical property of that system. This section briefly introduces the field theories that describe the fundamental interactions, as well as the importance of gauge invariance as it relates to the Higgs boson. 


\subsubsection{Quantum electrodynamics}

The interactions of spin- $1 / 2$ particles (denoted by the spinor $\psi$ ) of mass $m$ and charge $Q$ are described by the Dirac Lagrangian $[12,13]$ :

$$
\mathcal{L}_{\text {Dirac }}=\bar{\psi}\left(i \gamma^{\mu} \partial_{\mu}-m\right) \psi
$$

where $\gamma_{\mu}$ represents the Dirac matrices. Performing a local gauge transformation, i.e. one that depends on the position in space-time, $x$, of the form:

$$
\psi(x) \rightarrow e^{-i \theta(x)} \psi(x)
$$

where $\theta(x)$ is a real-valued function, yields the following transformation for the Lagrangian:

$$
\mathcal{L} \rightarrow \mathcal{L}-\left(\partial_{\mu} \theta\right) \bar{\psi} \gamma^{\mu} \psi
$$

In other words, it is not gauge invariant in this form. In order to achieve gauge invariance, it is necessary to introduce a massless vector field $A_{\mu}$ (the electromagnetic field), which transforms as:

$$
A_{\mu}(x) \rightarrow A_{\mu}(x)+\partial_{\mu} \theta(x)
$$

With the addition of this new field and defining the covariant derivative, which represents the minimal coupling of the photon to the spin- $1 / 2$ particles:

$$
D_{\mu}=\partial_{\mu}+i q A_{\mu}
$$

the Lagrangian takes its gauge-invariant form, known as the QED Lagrangian, where QED stands for quantum electrodynamics - the study of the interactions 
of massive, charged, spin-1/2 particles and the electromagnetic field. The QED Lagrangian is then:

$$
\mathcal{L}_{\mathrm{QED}}=\bar{\psi}\left(i \gamma^{\mu} D_{\mu}-m\right) \psi-\frac{1}{4} F_{\mu \nu} F^{\mu \nu}
$$

where $F_{\mu \nu}$ is the electromagnetic field tensor:

$$
F_{\mu \nu}=\partial_{\mu} A_{\nu}-\partial_{\nu} A_{\mu}
$$

In summary, in order to achieve the desired goal of gauge invariance, it was necessary to add a massless field; if the Lagrangian had included a mass term for $A_{\mu}$, it would not have been invariant under the transformation. The significance of this will become clearer later on, when the unification of the electromagnetic and weak interactions is discussed in Section 2.2.3. The transformation of Equation 2.2 can be thought of as a multiplication by a unitary $1 \times 1$ matrix of the $U(1)$ group. Hence, QED is symmetric under $U(1)$.

\subsubsection{Quantum chromodynamics}

Quantum chromodynamics (QCD) $[12,14]$ describes the interactions of quarks and gluons; i.e. the strong interaction. Since quarks are also massive, spin-1/2 particles, the starting point for the QCD Lagrangian is once again the Dirac Lagrangian given by Equation 2.1, but this time $\psi$ represents the quarks, which come in three 
colours (red, blue, and green), in terms of a column vector of Dirac spinors:

$$
\psi=\left(\begin{array}{l}
\psi_{r} \\
\psi_{b} \\
\psi_{g}
\end{array}\right) .
$$

In this case, the transformation takes the form:

$$
\psi \rightarrow e^{i \theta} e^{-i g \lambda \cdot \phi} \psi
$$

where the $\boldsymbol{\lambda}$ represent the eight $3 \times 3$ Gell-Mann matrices (since three quark colours are involved); this matrix group is known as the $S U(3)_{C}$ group, where the $C$ represents colour. The vector $\phi=-\boldsymbol{a} / g$ where $\boldsymbol{a}$ represents a vector of eight real numbers and $g$ represents the strong coupling constant. To achieve invariance under $S U(3)_{C}$ transformations, the derivative in the Dirac equation is replaced by the covariant derivative, which takes the form:

$$
D_{\mu}=\partial_{\mu}+i g \boldsymbol{\lambda} \cdot \boldsymbol{G}_{\boldsymbol{\mu}}
$$

where $\boldsymbol{G}_{\boldsymbol{\mu}}$ represents the introduction of eight massless fields corresponding to the eight gluons, which carry the various combinations of colour and anticolour in the strong interaction.

In its final, gauge-invariant form, the QCD Lagrangian is given by:

$$
\mathcal{L}_{\mathrm{QCD}}=\bar{\psi}^{j}\left(i \gamma^{\mu} D_{\mu}^{j k}-m^{j k}\right) \psi^{k}-\frac{1}{4} F_{\mu \nu}^{a} F_{a}^{\mu \nu}
$$


where $m$ represents the mass of a given quark flavour and the indices $j, k$ run from 1 to 3 (for the three quarks). The gluon field tensor $F_{\mu \nu}$ is defined as:

$$
F_{\mu \nu}^{a}=\partial_{\mu} G_{\nu}^{a}-\partial_{\nu} G_{\mu}^{a}-g f^{a b c} \lambda^{a} G_{\mu}^{b} G_{\nu}^{c}
$$

where the indices $a, b, c$ run from 1 to 8 (for the eight gluons); the $f_{a b c}$ represent the $S U(3)$ structure constants. The last term in Equation 2.12 permits triplet and quartic gluon self-couplings; this is possible since the gluons carry colour charge, unlike in QED, where the photon is electrically neutral.

\subsubsection{The electroweak interaction and the Standard Model}

Shortly following the Big Bang, in the time interval from about $10^{-36} \mathrm{~s}$ to about $10^{-12} \mathrm{~s}$, it is theorized that the electromagnetic and weak interactions were unified. This unified interaction, which occurs at energies on the order of $100 \mathrm{GeV}$, is known as the electroweak interaction [15-17].

In the electroweak theory, fermions are grouped according to their chirality and weak isospin $I_{3}$; the latter quantity is a conserved quantum number of the weak interaction, defined in terms of the electric charge $Q$ and the weak hypercharge $Y[13,14]:$

$$
I_{3}=Q-\frac{1}{2} Y
$$

There are three generations of both left- and right-handed quarks and leptons. The left-handed particles form weak isodoublets:

$$
L_{1}=\left(\begin{array}{c}
\nu_{e} \\
e
\end{array}\right)_{L}, L_{2}=\left(\begin{array}{c}
\nu_{\mu} \\
\mu
\end{array}\right)_{L}, L_{3}=\left(\begin{array}{c}
\nu_{\tau} \\
\tau
\end{array}\right)_{L}
$$




$$
Q_{1}=\left(\begin{array}{c}
u \\
d
\end{array}\right)_{L}, Q_{2}=\left(\begin{array}{l}
c \\
s
\end{array}\right)_{L}, Q_{3}=\left(\begin{array}{l}
t \\
b
\end{array}\right)_{L} .
$$

The right-handed particles form weak isosinglets:

$$
\begin{aligned}
& e_{R 1}=e_{R}, \quad e_{R 2}=\mu_{R}, e_{R 3}=\tau_{R}, \\
& u_{R 1}=u_{R}, u_{R 2}=c_{R}, u_{R 3}=t_{R}, \\
& d_{R 1}=d_{R}, d_{R 2}=s_{R}, d_{R 3}=b_{R} .
\end{aligned}
$$

For the gauge bosons, there are three fields $W_{\mu}^{1,2,3}$ corresponding to the $S U(2)_{L}$ group and one field $B_{\mu}$ corresponding to the $U(1)_{Y}$ group. The covariant derivative is defined as:

$$
D_{\mu}=\partial_{\mu}-i g_{s} \frac{\lambda_{a}}{2} G_{\mu}^{a}-i g_{2} \frac{\tau_{a}}{2} W_{\mu}^{a}-i g_{1} \frac{Y}{2} B_{\mu}
$$

where the $\tau_{a}$ are the three Pauli matrices (the $2 \times 2$ matrix generators of the $S U(2)_{L}$ group), $Y$ is the generator of the $U(1)_{Y}$ group, and $g_{s}, g_{2}$, and $g_{1}$ are the $S U(3)_{C}, S U(2)_{L}$, and $U(1)_{Y}$ coupling constants, respectively.

The field strengths are given by:

$$
\begin{gathered}
G_{\mu \nu}^{a}=\partial_{\mu} G_{\nu}^{a}-\partial_{\nu} G_{\mu}^{a}-g_{s} f^{a b c} G_{\mu}^{b} G_{\nu}^{c} \\
W_{\mu \nu}^{a}=\partial_{\mu} W_{\nu}^{a}-\partial_{\nu} W_{\mu}^{a}-g \epsilon^{a b c} W_{\mu}^{b} W_{\nu}^{c} \\
B_{\mu \nu}=\partial_{\mu} B_{\nu}-\partial_{\nu} B_{\mu},
\end{gathered}
$$

where $\epsilon^{a b c}$ represents the antisymmetric tensor. 
Combining the various pieces from the electroweak and QCD sectors gives the Standard Model Lagrangian, which is invariant under local $S U(3)_{C} \times S U(2)_{L} \times$ $U(1)_{Y}$ gauge transformations:

$$
\begin{aligned}
\mathcal{L}_{\mathrm{SM}} & =-\frac{1}{4} G_{\mu \nu}^{a} G_{a}^{\mu \nu}-\frac{1}{4} W_{\mu \nu}^{a} W_{a}^{\mu \nu}-\frac{1}{4} B_{\mu \nu} B^{\mu \nu} \\
& +\bar{L}_{i} i D_{\mu} \gamma^{\mu} L_{i}+\bar{e}_{R_{i}} i D_{\mu} \gamma^{\mu} e_{R_{i}}+\bar{Q}_{i} i D_{\mu} \gamma^{\mu} Q_{i}+\bar{u}_{R_{i}} i D_{\mu} \gamma^{\mu} u_{R_{i}}+\bar{d}_{R_{i}} i D_{\mu} \gamma^{\mu} d_{R_{i}} .
\end{aligned}
$$

It is important to note that the Lagrangian given in Equation 2.21 does not contain mass terms. In the examples of QED (Section 2.2.1) and QCD (Section 2.2.2), it was shown that in order for the Lagrangian to be gauge invariant, it was necessary to include massless fields corresponding to the gauge bosons. However, the gauge bosons of the weak interaction, the $W^{ \pm}$and the $Z$ bosons, are known to be massive $\left(m_{W^{ \pm}}=80.4 \mathrm{GeV}\right.$ and $\left.m_{Z}=91.2 \mathrm{GeV}[10]\right)$, as are the fermions. It is possible to add mass terms for the fermions in an $S U(3)_{C}$ invariant manner, but simply adding mass terms for the electroweak gauge bosons would violate the $S U(2)_{L} \times U(1)_{Y}$ symmetry. Hence, the problem which troubled theorists during the development of the electroweak theory was: how could this theory be modified to take into account the masses of the gauge bosons and fermions while still maintaining $S U(2)_{L} \times U(1)_{Y}$ invariance? The solution to this problem lies in the mechanism of spontaneous electroweak symmetry breaking. 


\subsection{Spontaneous symmetry breaking: the Higgs mechanism}

The concept of electroweak symmetry breaking was developed in the 1960s by Peter Higgs [18, 19]; François Englert and Robert Brout [20]; and Gerald Guralnik, Carl Hagen, and Tom Kibble [21].

The electroweak part of the Standard Model Lagrangian, given by Equation 2.21, is [14]:

$$
\mathcal{L}_{E W}=-\frac{1}{4} W_{\mu \nu}^{a} W_{a}^{\mu \nu}-\frac{1}{4} B_{\mu \nu} B^{\mu \nu}+\bar{L}_{i} i D_{\mu} \gamma^{\mu} L_{i}+\bar{e}_{R_{i}} i D_{\mu} \gamma^{\mu} e_{R_{i}} .
$$

In order to generate the masses of the electroweak gauge bosons, it is necessary to introduce an $S U(2)_{L}$ doublet of scalar fields:

$$
\Phi=\left(\begin{array}{c}
\phi^{+} \\
\phi^{0}
\end{array}\right) .
$$

The invariant Lagrangian for the scalar fields is given by:

$$
\begin{aligned}
\mathcal{L}_{S} & =\left(D^{\mu} \Phi\right)^{\dagger}\left(D_{\mu} \Phi\right)-V(\Phi) \\
& =\left(D^{\mu} \Phi\right)^{\dagger}\left(D_{\mu} \Phi\right)-\mu^{2} \Phi^{\dagger} \Phi-\lambda\left(\Phi^{\dagger} \Phi\right)^{2},
\end{aligned}
$$

where $\lambda>0$ so that the potential $V$ is bounded from below. If the parameter $\mu^{2}<0$, the ground state of $\Phi$ has a non-zero value:

$$
\langle\Phi\rangle_{0}=\left(\begin{array}{c}
0 \\
\frac{v}{\sqrt{2}}
\end{array}\right),
$$


where $v$ denotes the vacuum expectation value ("vev"):

$$
v=\sqrt{-\frac{\mu^{2}}{\lambda}}
$$

Since the vev is non-zero, the $S U(2)_{L} \times U(1)_{Y}$ symmetry is spontaneously broken.

The physical implications of this spontaneous symmetry breaking can be seen if the field $\Phi$ is expressed in terms of four fields $\theta_{a=1,2,3}(x)$ and $H(x)$ :

$$
\Phi(x)=e^{i \theta_{a}(x) \tau^{a} / v}\left(\begin{array}{c}
0 \\
\frac{1}{\sqrt{2}}(v+H(x))
\end{array}\right),
$$

where the $\tau_{a}$ are the three non-commuting $2 \times 2$ Pauli matrices. In order to use the more convenient unitary gauge a transformation is applied:

$$
\Phi(x) \rightarrow e^{-i \theta_{a}(x) \tau^{a} / v} \Phi(x)=\frac{1}{\sqrt{2}}\left(\begin{array}{c}
0 \\
v+H(x)
\end{array}\right)
$$

Then, expanding the $\left|D_{\mu} \Phi\right|^{2}$ term of Equation 2.24 about the ground state of $\Phi$ defined in Equation 2.28:

$$
\begin{aligned}
\left|D_{\mu} \Phi\right|^{2} & =\left|\left(\partial_{\mu}-i g_{2} \frac{\tau_{a}}{2} W_{\mu}^{a}-i g_{1} \frac{1}{2} B_{\mu}\right) \Phi\right|^{2} \\
& =\frac{1}{2}\left|\left(\begin{array}{cc}
\partial_{\mu}-\frac{i}{2}\left(g_{2} W_{\mu}^{3}+g_{1} B_{\mu}\right) & -\frac{i g_{2}}{2}\left(W_{\mu}^{1}-i W_{\mu}^{2}\right) \\
-\frac{i g_{2}}{2}\left(W_{\mu}^{1}+i W_{\mu}^{2}\right) & \partial_{\mu}+\frac{i}{2}\left(g_{2} W_{\mu}^{3}-g_{1} B_{\mu}\right)
\end{array}\right)\left(\begin{array}{c}
0 \\
v+H
\end{array}\right)\right|^{2} \\
& =\frac{1}{2}\left(\partial_{\mu} H\right)^{2}+\frac{1}{8} g_{2}^{2}(v+H)^{2}\left|W_{\mu}^{1}+i W_{\mu}^{2}\right|^{2}+\frac{1}{8}(v+H)^{2}\left|g_{2} W_{\mu}^{3}-g_{1} B_{\mu}\right|^{2} .
\end{aligned}
$$


If Equation 2.29 is expanded completely and the fields corresponding $W^{ \pm}$ and $\mathrm{Z}$ bosons and the photon are defined according to:

$$
W_{\mu}^{ \pm}=\frac{1}{\sqrt{2}}\left(W_{\mu}^{1} \mp i W_{\mu}^{2}\right), Z_{\mu}=\frac{g_{2} W_{\mu}^{3}-g_{1} B_{\mu}}{\sqrt{g_{2}^{2}+g_{1}^{2}}}, A_{\mu}=\frac{g_{2} W_{\mu}^{3}+g_{1} B_{\mu}}{\sqrt{g_{2}^{2}+g_{1}^{2}}},
$$

it is then possible to collect mass terms of the form $M_{W^{ \pm}}^{2} W_{\mu}^{+} W^{-\mu}, \frac{1}{2} M_{Z}^{2} Z_{\mu} Z^{\mu}$, and $\frac{1}{2} M_{A}^{2} A_{\mu} A^{\mu}$. These terms yield the following relations:

$$
M_{W^{ \pm}}=\frac{1}{2} g_{2} v, M_{Z}=\frac{1}{2} v \sqrt{g_{2}^{2}+g_{1}^{2}}, M_{A}=0 .
$$

Thus, the non-zero vev of the scalar field spontaneously breaks the symmetry: the three degrees of freedom corresponding to $\theta_{1,2,3}(x)$ are absorbed by the $W^{ \pm}$and $Z$ bosons, allowing them to acquire masses, while the photon remains massless. This is, of course, the Higgs mechanism, with the remaining degree of freedom, the scalar $H$, corresponding to the Higgs field.

The Higgs mechanism can also be used to give mass to the fermions. In the following example of the electron, the $S U(2)_{L} \times U(1)_{Y}$ invariant Yukawa Lagrangian is:

$$
\mathcal{L}_{F}=-\lambda_{e} \bar{L} \Phi e_{R}+h . c .
$$

where $h$. c. represents the Hermitian conjugate. Expanding about the vev as before:

$$
\begin{aligned}
\mathcal{L}_{F} & =-\frac{1}{\sqrt{2}} \lambda_{e}\left(\bar{\nu}_{e}, \bar{e}_{L}\right)\left(\begin{array}{c}
0 \\
v+H
\end{array}\right) e_{R}+\ldots \\
& =-\frac{1}{\sqrt{2}} \lambda_{e}(v+H) \bar{e}_{L} e_{R}+\ldots
\end{aligned}
$$

The procedure is identical for the other massive fermions (neutrinos are treated as 
massless particles in the Standard Model). Then, the $\bar{f}_{L} f_{R}$ terms give the fermion masses:

$$
m_{e}=\frac{\lambda_{e} v}{\sqrt{2}}, m_{u}=\frac{\lambda_{u} v}{\sqrt{2}}, m_{d}=\frac{\lambda_{d} v}{\sqrt{2}}
$$

Note that this does not allow for precise predictions of the masses, since the values of the $\lambda$ coupling constants are not explicitly defined in the Standard Model.

The couplings of the Higgs boson to gauge bosons $(V)$ and fermions $(f)$ can be obtained from Equation 2.29 and Equation 2.33 by identifying the terms of order $H$ and $H^{2}$ :

$$
g_{H V V}=-2 i \frac{M_{V}^{2}}{v}, g_{H H V V}=-2 i \frac{M_{V}^{2}}{v^{2}}, g_{H f f}=i \frac{m_{f}}{v} .
$$

If the same procedure as that used for the gauge boson and fermion masses is attempted in order to determine the mass of the Higgs boson, it is necessary to expand the scalar potential about the vev as follows:

$$
\begin{aligned}
V(\Phi) & =\mu^{2} \Phi^{\dagger} \Phi+\lambda\left(\Phi^{\dagger} \Phi\right)^{2} \\
& =\frac{\mu^{2}}{2}(0, v+H)\left(\begin{array}{c}
0 \\
v+H
\end{array}\right)+\frac{\lambda}{4}\left|(0, v+H)\left(\begin{array}{c}
0 \\
v+H
\end{array}\right)\right|^{2} \\
& =-\frac{1}{2} \lambda v^{2}(v+H)^{2}+\frac{1}{4} \lambda(v+H)^{4} .
\end{aligned}
$$

The Lagrangian for the Higgs field $H$ is then:

$$
\begin{aligned}
\mathcal{L}_{H} & =\frac{1}{2}\left(\partial_{\mu} H\right)\left(\partial^{\mu} H\right)-V \\
& =\frac{1}{2}\left(\partial^{\mu} H\right)^{2}-\lambda v^{2} H^{2}-\lambda v H^{3}-\frac{\lambda}{4} H^{4} .
\end{aligned}
$$


Attempting to pick out a mass term of the form $\frac{1}{2} M_{H}^{2}$ yields the relation:

$$
M_{H}^{2}=2 \lambda v^{2}=-2 \mu^{2} .
$$

The value of the vev can be obtained from the known Fermi coupling constant, $G_{F}:$

$$
v=\left(\sqrt{2} G_{F}\right)^{-1 / 2} \approx 246 \mathrm{GeV} .
$$

However, the parameter $\lambda$ is not explicitly defined in the Standard Model. Hence, the mass of the Higgs boson cannot be predicted theoretically and must be determined experimentally.

\subsection{Previous experimental searches for the Higgs boson}

Prior to the inauguration of the LHC, other experimental searches set limits on the Higgs boson's mass.

CERN's Large Electron-Positron Collider (LEP), which ran from 1989 - 2000, searched for the Higgs boson produced in association with a $Z$ boson $\left(e^{+} e^{-} \rightarrow Z H\right)$. Its final run, at centre-of-mass energies ranging from $\sqrt{s}=$ $189 \mathrm{GeV}$ to $209 \mathrm{GeV}$, found no significant evidence for the existence of the Higgs boson, and set a lower limit of $114.4 \mathrm{GeV}$ on its mass at the $95 \%$ confidence level [22].

Additional searches took place at Fermilab's Tevatron, a proton-antiproton collider which ran from 1987 - 2011 at centre-of-mass energies of up to $\sqrt{s}=$ $1.96 \mathrm{TeV}$. The Tevatron focussed on the following production modes: $g g \rightarrow H$, 
$q \bar{q} \rightarrow W H, Z H$, and $q \bar{q} \rightarrow q \bar{q} H$. At the time of the discovery of the Higgs boson at the LHC in 2012, the Tevatron had excluded the mass range $147 \mathrm{GeV}<m_{H}<$ $180 \mathrm{GeV}[23]$.

Indirect limits on the Higgs boson's mass were also formulated using the results of electroweak precision measurements performed at LEP and the Tevatron. Since the Higgs boson is involved in loop corrections in the electroweak sector, the measured values of the masses of the electroweak gauge bosons and the fermions (in particular the $W$ boson and the top quark) can be used to constrain the mass of the Higgs boson. The result of the global fit to the most recent (as of 2011) measured electroweak parameters [24] yielded the following limit on the Higgs boson's mass: $m_{H}=96_{-23}^{+30} \mathrm{GeV}$. Taking into account the exclusion limits obtained via direct searches yielded a revised limit of $m_{H}=120_{-5}^{+12} \mathrm{GeV}$.

\subsection{Higgs boson searches at the LHC}

\subsubsection{Hadron collider physics}

At the LHC, Higgs boson production occurs as a result of interactions between colliding protons. In the parton model developed by Richard Feynman [25], protons are composed of fundamental constituents collectively denoted as "partons." In addition to three "valence" quarks (uud), protons also contain gluons and a "sea" of virtual quarks and antiquarks. The partons are described by parton distribution functions (PDFs), which give the probability density of finding a particle with a momentum fraction $x$ of the proton's momentum. At hadron colliders, such as the LHC, "hard scattering" processes involving high momentum transfer between partons result in the production of new particles. An example of Higgs boson 
production resulting from such a process is depicted in Figure 2.1. In addition to the hard scattering process, "soft" interactions between the remaining partons can also occur; these make up what is known as the underlying event.

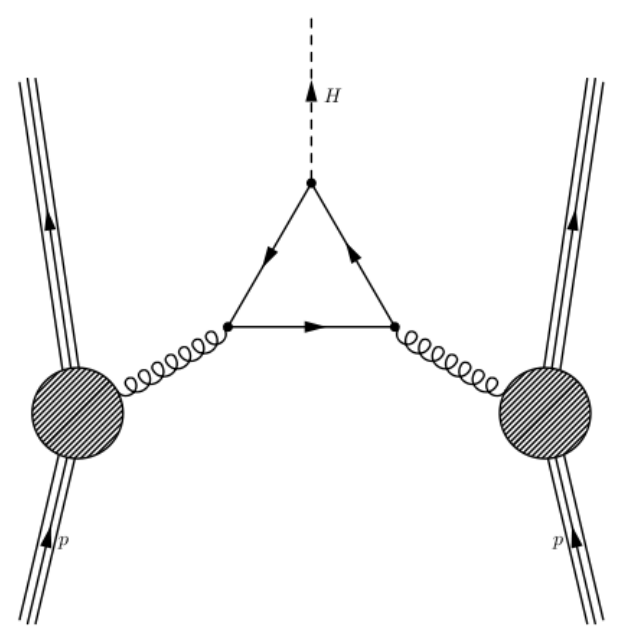

Figure 2.1: Schematic of a proton-proton collision at the LHC, depicting the three valence quarks of the protons. The interaction of the protons' constituent partons (gluons, in this case) results in the production of a Higgs boson.

\subsubsection{Production modes}

Since there are many ways in which the partons can interact, several possible production mechanisms exist; each with its own unique experimental signature. The most important production mechanisms are illustrated in Figure 2.2 and include the following, in order of prevalence:

- Gluon-gluon fusion (ggF) Two gluons "fuse" via a quark loop (preferentially a top quark loop, since the Higgs boson coupling to fermions is proportional to the fermion mass, as shown in Equation 2.35), and a Higgs boson is emitted. 
- Vector boson fusion (VBF) Two quarks emit $W$ or $Z$ bosons, which then fuse and emit a Higgs boson. In this process, the Higgs boson is accompanied by a characteristic dijet signature in the detector from the two quarks.

- Associated production with a vector boson (VH) A quark and antiquark interact to produce a $W$ or $Z$ boson, which then emits a Higgs boson. The vector boson decays either leptonically or hadronically, providing an additional characteristic signature in the detector.

- Associated production with a $t \bar{t}$ pair $(t \bar{t} \mathbf{H})$ A quark from a $t \bar{t}$ pair fuses with an antiquark from another pair, producing a Higgs boson. The remaining quark and antiquark hadronize, and the interactions of the hadrons in the detector results in jet production.

The cross-sections for Higgs boson production are calculated perturbatively. Leading order (LO) calculations take into account only the simplest tree-level diagram associated with the process. The calculation may be refined by including higher-order terms; for example, next-to-leading order (NLO) QCD calculations include corrections of order $\alpha_{S}$ (the strong coupling constant), corresponding to the radiation of a gluon. Similarly, electroweak corrections corresponding to $W$ or $Z$ boson emission may also be taken into account. Figure 2.3 shows the Higgs boson production cross-sections for the four mechanisms at the LHC, for $\sqrt{s}=7 \mathrm{TeV}$ and $\sqrt{s}=8 \mathrm{TeV}$.

\subsubsection{Decay modes}

The Higgs boson is unstable; once produced, it decays almost immediately at the interaction point and is identified experimentally by searching for the signatures of 


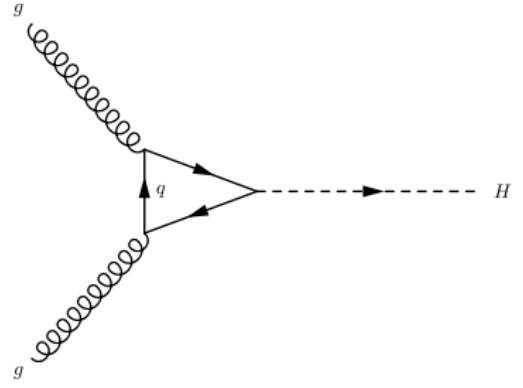

(a) $g g \rightarrow H$

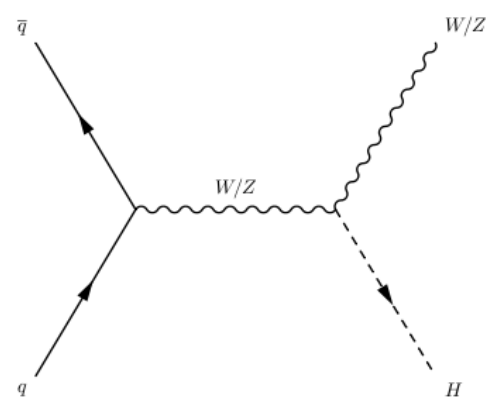

(c) $q \bar{q} \rightarrow V+H$

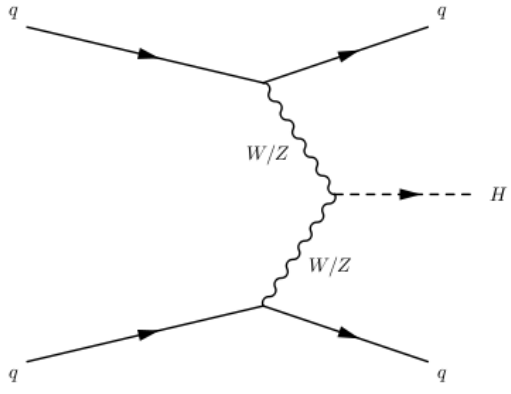

(b) $q q \rightarrow q q+H$

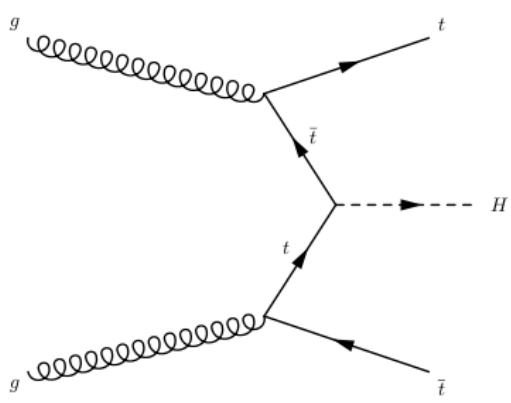

(d) $g g \rightarrow t \bar{t}+H$

FiguRE 2.2: Higgs boson production mechanisms at the LHC: (a) gluon-gluon fusion, (b) vector boson fusion, (c) associated production with a vector boson, and (d) associated production with a $t \bar{t}$ pair.

its decay products in the detector. Figure 2.4 shows the production cross-section, multiplied by the branching ratio of the decay process, for the various Higgs boson decay modes at the LHC.

One possible decay mode is the $H \rightarrow Z Z^{(*)} \rightarrow 4 \ell$ channel, in which the Higgs boson decays to a pair of $Z$ bosons (for $M_{H}<2 M_{Z}$, one of the $Z$ bosons is a virtual particle), which subsequently decay leptonically, where the leptons may be $e^{+} e^{-}$or $\mu^{+} \mu^{-}$. This channel, which features an experimental signature of four well-isolated leptons with relatively large transverse momentum, is often called the "golden channel". Although it is a relatively rare process, the $H \rightarrow Z Z^{(*)} \rightarrow 4 \ell$ decay is easier to identify than other more common decay modes which feature 


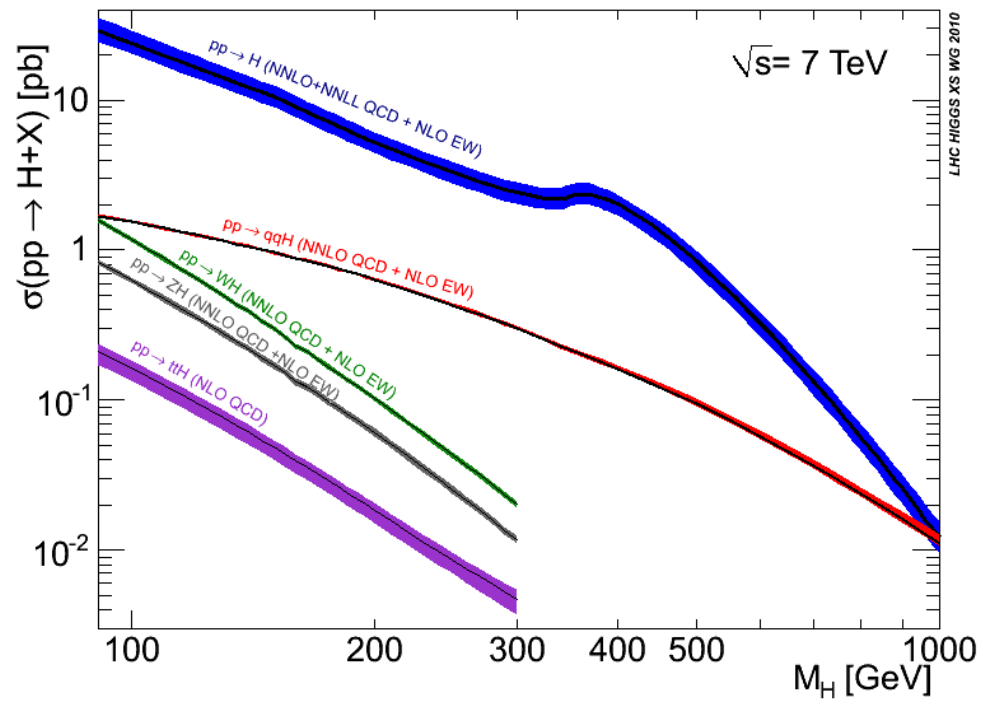

(a) $\sqrt{s}=7 \mathrm{TeV}$

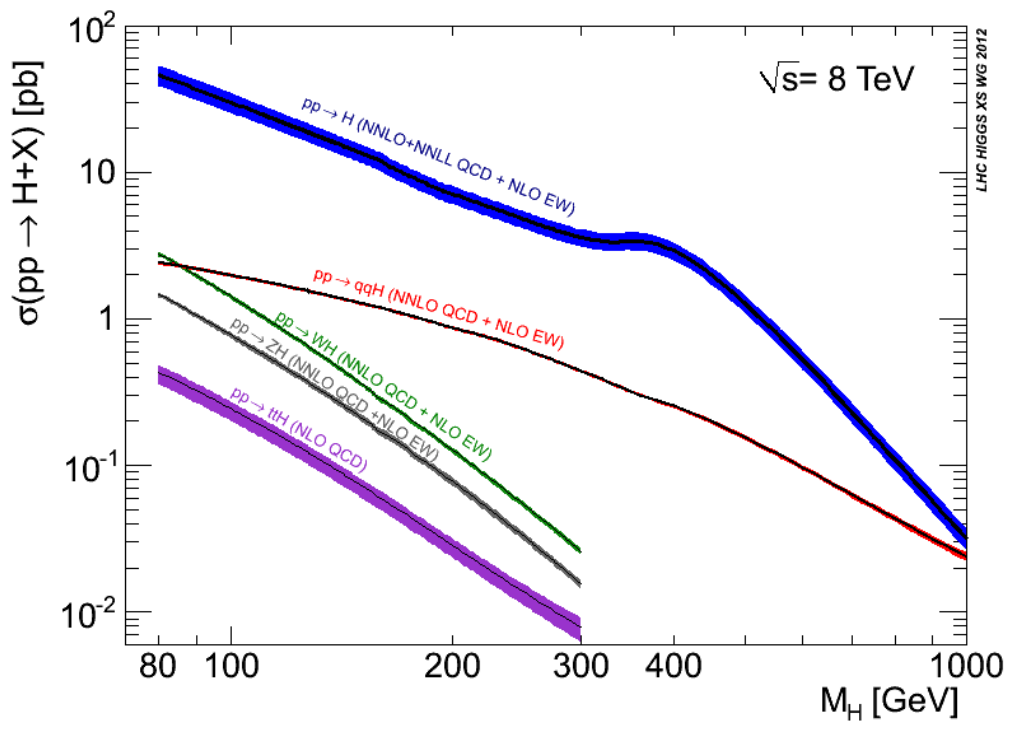

(b) $\sqrt{s}=8 \mathrm{TeV}$

Figure 2.3: Theoretical Higgs boson production cross-sections as a function of the Higgs boson mass. The cross-sections are calculated for gluon-gluon fusion ( $p p \rightarrow H$; in blue), vector boson fusion ( $p p \rightarrow q q H$; in red), and associated production with a $W$ boson, $Z$ boson, and $t \bar{t}$ pair $(p p \rightarrow W H, p p \rightarrow Z H$, and $p p \rightarrow t \bar{t} H$; in green, grey, and purple, respectively). The predictions are shown for proton-proton collisions at (a) $\sqrt{s}=7 \mathrm{TeV}$ and (b) $\sqrt{s}=8 \mathrm{TeV}$ at the LHC [26]. 


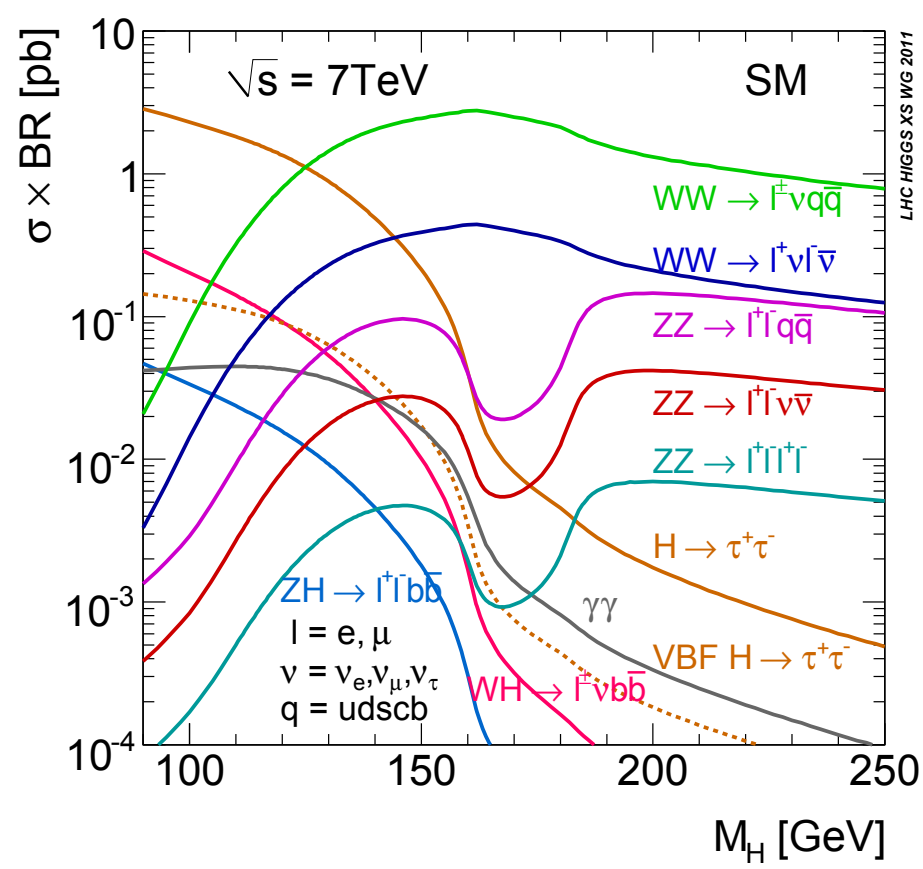

(a) $\sqrt{s}=7 \mathrm{TeV}$

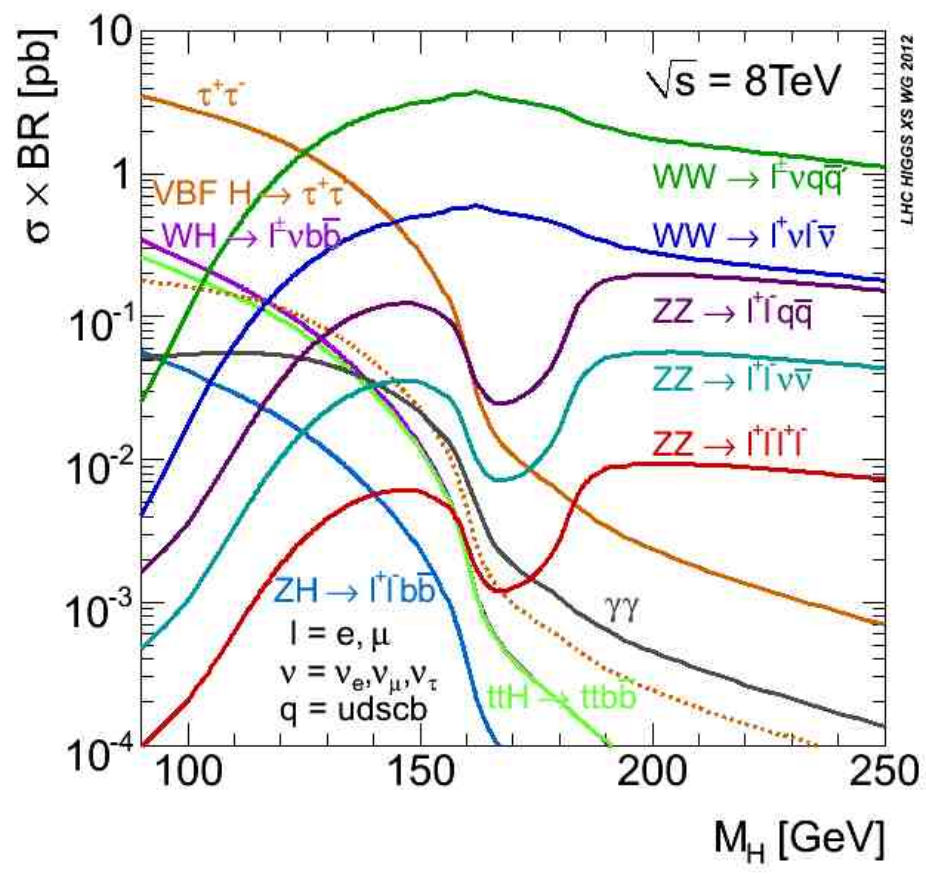

(b) $\sqrt{s}=8 \mathrm{TeV}$

FIgURE 2.4: Theoretical Higgs boson production cross-sections, multiplied by the branching ratio for its decay modes, as a function of the Higgs boson mass. The predictions are shown for proton-proton collisions at (a) $\sqrt{s}=7 \mathrm{TeV}$ and (b) $\sqrt{s}=8 \mathrm{TeV}$ at the LHC [26]. 
hadrons as the final products, since those processes tend to be obscured by the significant Standard Model jet backgrounds in the detector. The main source of background in the $H \rightarrow Z Z^{(*)} \rightarrow 4 \ell$ process is Standard Model $Z Z^{(*)}$ production, which is well-understood and can be accurately modelled. In addition, the presence of four leptons in the final state allows for precise reconstruction and measurement of the Higgs boson's mass. Note that this channel does not include $\tau$-leptons in the final state, since they subsequently decay and are difficult to distinguish from the background; in addition, since neutrinos are emitted in $\tau$ decays, the invariant mass of the Higgs boson cannot be reconstructed completely.

\subsection{Conclusion}

The Higgs boson is a critical component of the Standard Model of particle physics. The Higgs mechanism of spontaneous electroweak symmetry breaking was postulated in order to explain the process through which the gauge bosons and fermions acquire their masses. This mechanism also implies the existence of a massive scalar: the Higgs boson, whose mass is not predicted by the theory.

The search for the Higgs boson and the measurement of its properties is one of the main components of the physics programme at the Large Hadron Collider, which is capable of producing Higgs bosons via several mechanisms. The ATLAS experiment searches for the Higgs boson in a variety of decay channels; this work focuses on the study of the $H \rightarrow Z Z^{(*)} \rightarrow 4 \ell$ channel, which, although relatively rare, boasts a clean experimental signal. The theoretical aspects of the Higgs mechanism and the Higgs boson have been briefly outlined in this chapter; the remaining chapters discuss the experimental techniques used to search for search for the Higgs boson in the $H \rightarrow Z Z^{(*)} \rightarrow 4 \ell$ channel and measure its properties. 


\section{The LHC and the ATLAS Experiment}

Gigantic multiplied by colossal multiplied by staggeringly huge is the sort of concept we're trying to get across here.

- Douglas Adams, The Restaurant at the End of the Universe

\footnotetext{
(6)

$\mathrm{N}$ the context of this work, the Higgs boson is produced in proton-proton 28

e collisions. Section 3.1 introduces the machine which provides those collisions, the Large Hadron Collider, and Section 3.2 introduces ATLAS, the detector which measures the properties of the final-state particles resulting from the decays of Higgs bosons and other processes. In Section 3.3, the reconstruction of particles by combining information from the subdetectors will be discussed, and in Section 3.4, the framework used to simulate the interaction of particles with the ATLAS detector will be summarized.
}

\subsection{The Large Hadron Collider}

The Large Hadron Collider (LHC) [27, 28] is the largest and most powerful particle accelerator constructed to date. Measuring $26.7 \mathrm{~km}$ in circumference and located 
approximately $100 \mathrm{~m}$ below ground, straddling Swiss and French territory just outside the city of Geneva, the LHC is the crowning achievement of CERN, the European Organization for Nuclear Research [29]. The objective of this machine is ambitious: to produce vast amounts of data so that its associated experiments may perform precision measurements of the Standard Model, to search for and study the Higgs boson, and to explore physics beyond the Standard Model.

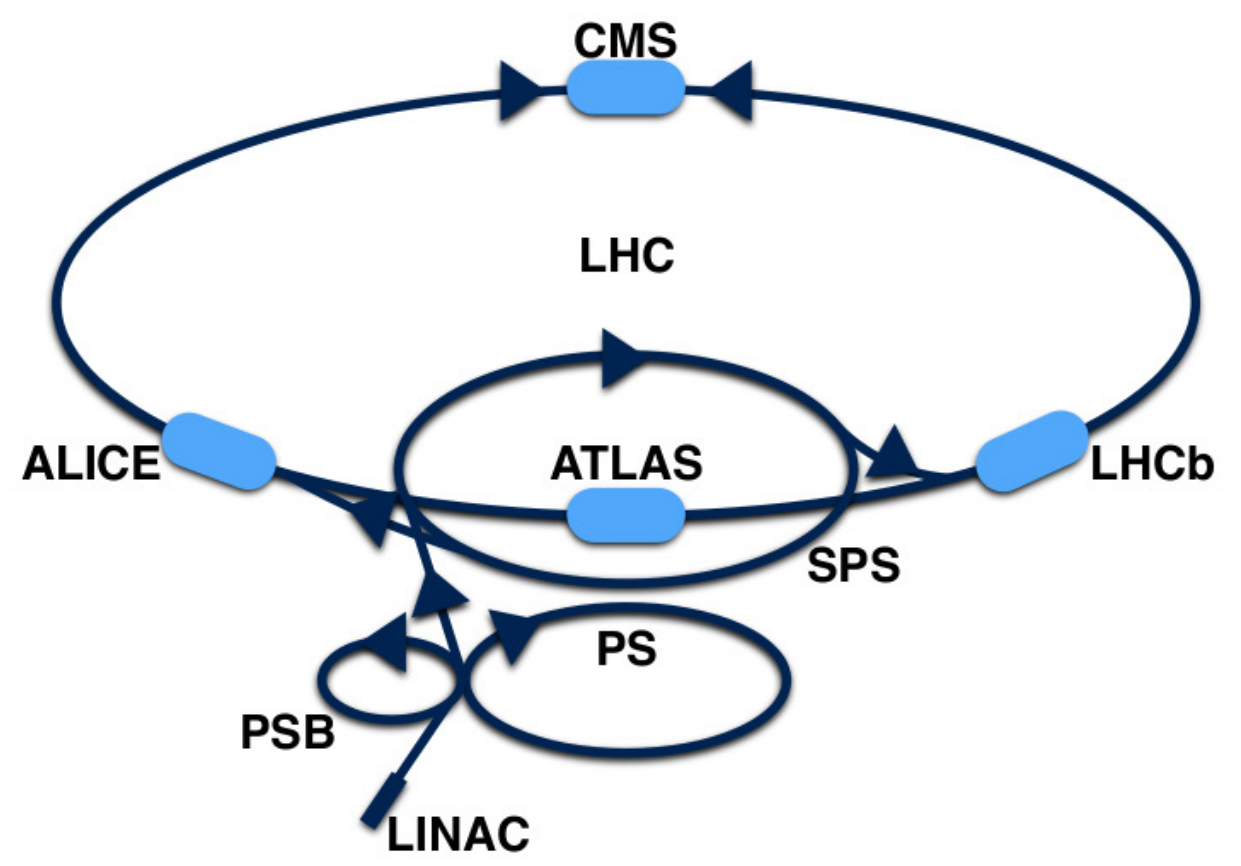

Figure 3.1: Artist's rendition of the CERN accelerator complex, inspired by [27]. The arrows indicate the directions of the proton beams.

Pictured in Figure 3.1, the LHC is the last stage of the CERN accelerator complex, consisting of a series of progressively larger and more powerful accelerators, which gradually accelerate beams of protons to the design energy of $7 \mathrm{TeV}$ per beam. The first step of this multi-stage injection chain is the linear accelerator, Linac2, which accelerates the protons to $50 \mathrm{MeV}$. From there, the beam is 
injected into the Proton Synchrotron Booster (PSB), where it attains an energy of $1.4 \mathrm{GeV}$. The beam then moves to the Proton Synchrotron (PS) until it is accelerated to $25 \mathrm{GeV}$, and then it travels to the Super Proton Synchrotron (SPS). Upon leaving the SPS and entering the LHC, the beam energy is $450 \mathrm{GeV}$.

Once in the LHC, the protons are contained in two separate beam pipes, where a system of superconducting dipole magnets steers the two beams in opposite directions, and quadrupole magnets serve to focus the beams. The beam pipes and magnets are contained in a cryostat cooled to below $2 \mathrm{~K}$ by superfluid helium. Radio-frequency cavities boost the beams' energies up to the design energy of $7 \mathrm{TeV}$ per beam over a period of approximately 20 minutes.

The beams collide at four interaction points corresponding to four major experiments. Two general-purpose experiments, ATLAS (A Toroidal LHC Apparatus) [30] and CMS (Compact Muon Solenoid) [31] have been designed to search for and study a broad range of Standard Model and new physics processes. LHCb [32] aims to study the physics processes associated with the $b$-quark. In addition to its main purpose as a proton-proton collider, the LHC is also a heavy ion collider. It is capable of colliding lead ions at energies up to $5.5 \mathrm{TeV}$ per nucleon pair; ALICE (A Large Ion Collider Experiment) [33] is dedicated to studying the results of these collisions. The work described in this thesis was performed as part of the ATLAS experiment.

Shortly after the first beams began circulating in 2008, the LHC experienced a significant setback. During the infamous "incident of September 19" [34], an electrical arc from a faulty connection between two of the superconducting magnets resulted in a sudden quench; helium was released and several magnets were seriously damaged. The LHC was immediately shut down for repairs. Operations resumed in 2009; for the initial period of high-energy collisions in 2010 
and 2011, it was decided to run at a reduced energy of $3.5 \mathrm{TeV}$ per beam until 2012, when the beam energy was increased to $4 \mathrm{TeV}$, for a total centre-of-mass energy of $\sqrt{s}=8 \mathrm{TeV}$. Early in 2013, the LHC was shut down for upgrade work, in order to bring the beam energy to the design specification of $7 \mathrm{TeV}$ per beam $(\sqrt{s}=14 \mathrm{TeV})$; it is expected that collisions will begin again early in 2015 at $\sqrt{s}=13 \mathrm{TeV}$.

The proton beams consist of 2808 bunches of $10^{11}$ protons per bunch; when the LHC operates at its full design specification, the bunches will cross every 25 ns. For the 2011 - 2012 run period, which provided the data used in this work, the minimum bunch spacing was $50 \mathrm{~ns}[35]$.

A key property of a proton-proton collider is its luminosity: the rate of inelastic collisions per unit area. For a collider with beams travelling at a revolution frequency $f_{r}, n_{b}$ bunch pairs colliding per revolution, a mean number of $\mu$ inelastic collisions per bunch crossing, and an inelastic cross-section $\sigma_{\text {inel }}$, the luminosity is given by:

$$
\mathcal{L}=\frac{\mu n_{b} f_{r}}{\sigma_{\text {inel }}}
$$

At the LHC's design luminosity of $10^{34} \mathrm{~cm}^{-2} \cdot \mathrm{s}^{-1}$, collisions will occur with a frequency of $40 \mathrm{MHz}$. Figure 3.2 shows the increasing peak instantaneous luminosity delivered by the LHC during the 2010, 2011, and 2012 run periods. The mean number of interactions per bunch crossing, $\mu$, which increased as a function of the instantaneous luminosity from year to year, is shown in Figure 3.3.

The integrated luminosity, i.e. the sum of the luminosity delivered over time, is shown for 2011 and 2012 in Figure 3.4. In 2011 (2012), the LHC delivered $5.46 \mathrm{fb}^{-1}\left(22.8 \mathrm{fb}^{-1}\right)$, of which $5.08 \mathrm{fb}^{-1}\left(21.3 \mathrm{fb}^{-1}\right)$ were recorded. The 


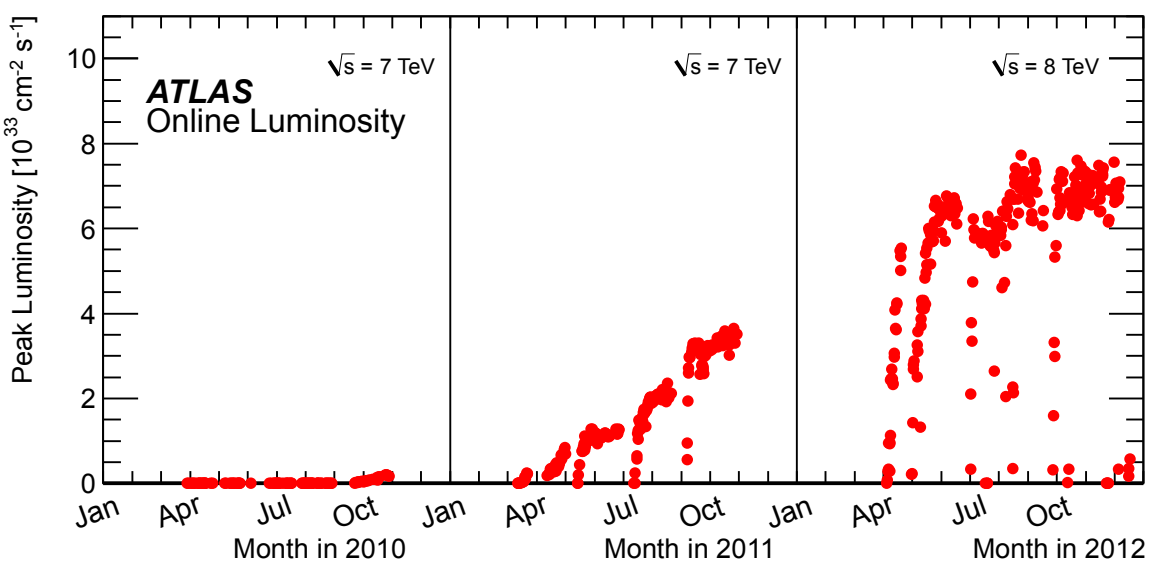

Figure 3.2: Peak instantaneous luminosity delivered to ATLAS by the LHC in 2010, 2011, and 2012 [36].

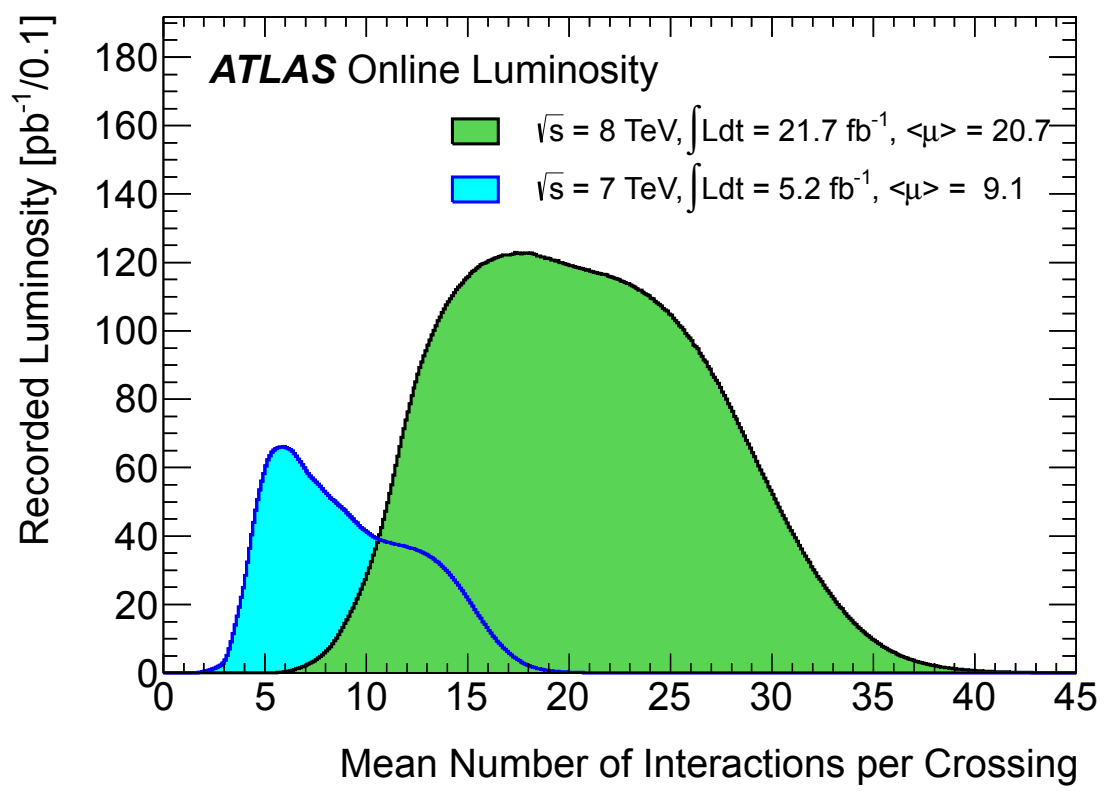

FiguRE 3.3: The mean number of interactions per bunch crossing in 2011 and 2012. In $2010,<\mu>\simeq 2[36]$. 
discrepancy between delivered and recorded luminosity is due to the data acquisition inefficiency, as well as the fact that certain subdetectors are only turned on after stable beams have been achieved. Of this recorded luminosity, $4.57 \mathrm{fb}^{-1}$ $\left(20.3 \mathrm{fb}^{-1}\right)$ were declared to be good for physics; i.e., the ATLAS detector subsystems were functioning free of any problems that could interfere with the quality of the recorded data.

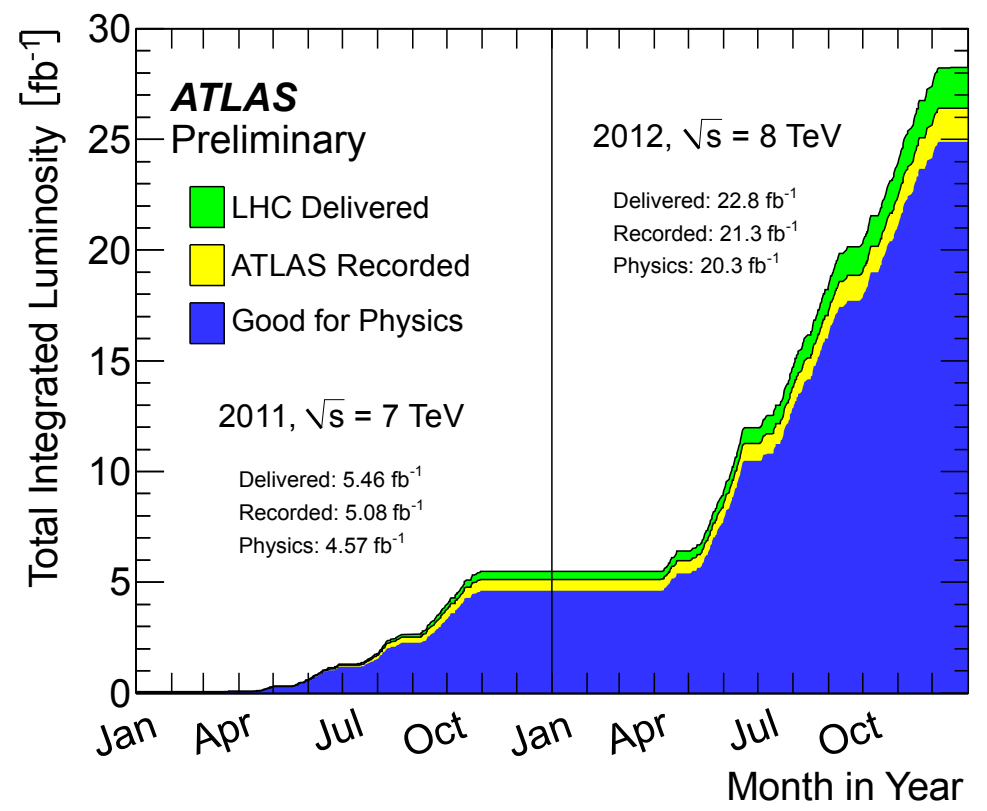

FiguRE 3.4: Integrated luminosity delivered by the LHC, recorded by ATLAS, and certified as good for physics analyses in 2011 and 2012 [36].

\subsection{The ATLAS Experiment}

\subsubsection{Motivation and physics program}

The unprecedented centre-of-mass energy and luminosity at the LHC necessitates equally unprecedented levels of technological innovation in its associated 
experiments. ATLAS has been designed to profit from the increased production cross-sections at the LHC in order to perform precise measurements and tests of the Standard Model, including QCD, electroweak interactions, and heavy flavour physics with $b-$ and $t$-quarks. Additionally, the ATLAS experiment searches for new, exotic particles and interactions beyond the scope of the Standard Model.

At the forefront of the ATLAS physics program is the search for the Higgs boson. For a low-mass Higgs boson, the $H \rightarrow Z Z^{(*)} \rightarrow 4 \ell$ and $H \rightarrow \gamma \gamma$ decay channels provide the best discovery potential and require excellent electromagnetic calorimetry and charged-particle tracking in order to identify leptons and photons produced in the final state. Jet identification and measurement is necessary to study hadronic decay modes, as well as to identify background events in the leptonic decay modes. In the wake of a discovery, precision measurements of the new particle require excellent instrumental resolution, since the width of the Higgs boson is predicted to be on the order of a few $\mathrm{MeV}$ at masses below $2 m_{Z}$, where $m_{Z}$ is the mass of the $Z$ boson $(91.2 \mathrm{GeV})$. All of the mentioned decay modes are important in Higgs boson searches and studies, in order to carefully measure its properties and discern whether it belongs to the Standard Model, or to a new regime of physics beyond the Standard Model.

\subsubsection{Overview of the ATLAS Experiment}

ATLAS, pictured in Figure 3.5, measures $44 \mathrm{~m}$ long, $25 \mathrm{~m}$ high, and weighs approximately 7,000 tonnes. It consists of multiple layers of subdetectors, each of which have been custom-designed to measure the energies and momenta of specific 
types of particles produced during proton-proton collisions at the centre of the detector. ATLAS is divided into a barrel region, consisting of concentric cylinders, and two end-caps with disk-shaped subdetectors to maximize coverage.

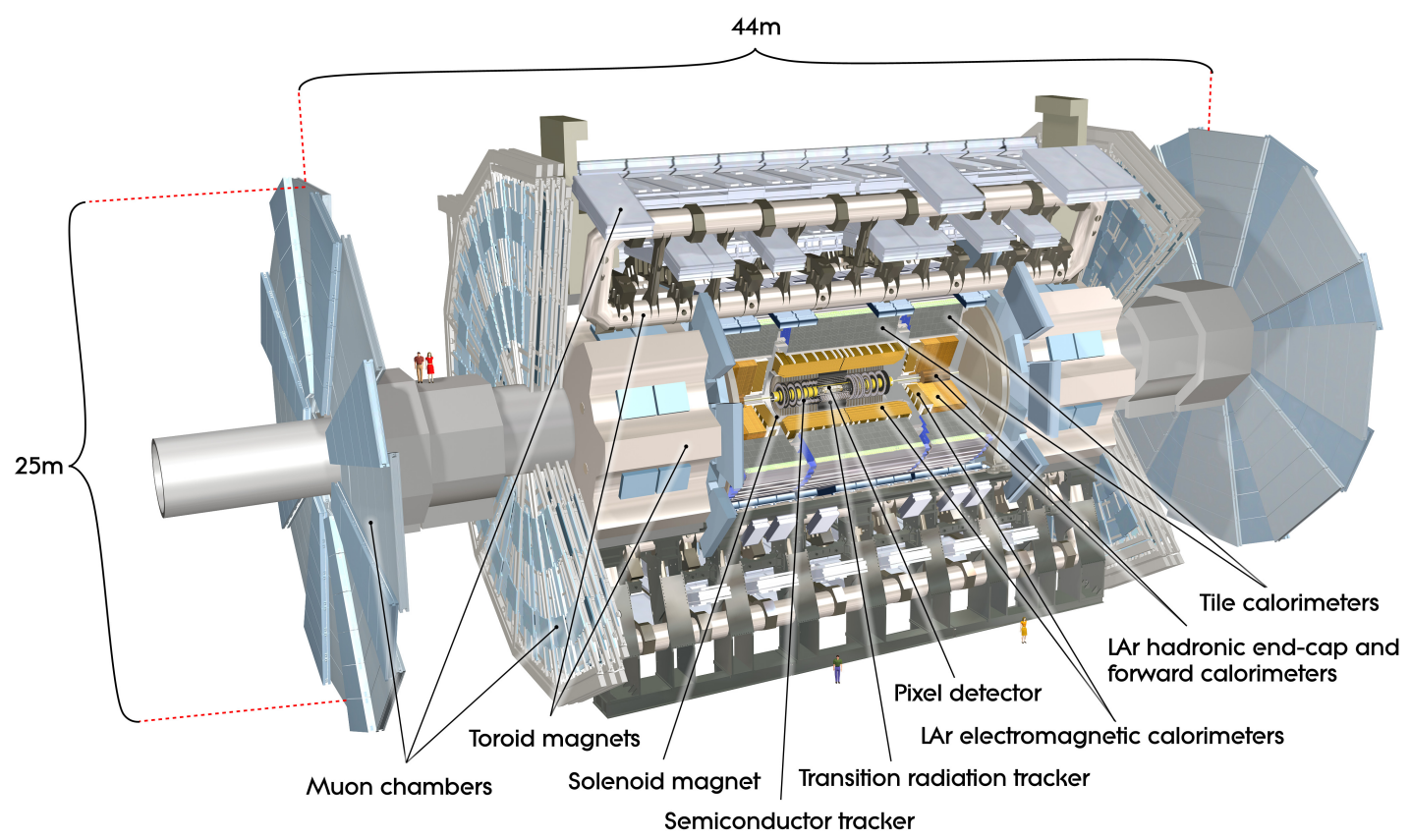

Figure 3.5: Cutaway view of the ATLAS detector, showing its concentric layers of subdetectors [30].

The high luminosity and particle production cross-sections require fast, radiation-hard electronics and highly granular subdetectors in order to withstand the high particle flux rates and to minimize the effects of pileup. Two types of pileup are possible; in-time pileup refers to multiple collisions occurring in a given bunch crossing, while out-of-time pileup occurs when signals from collisions in adjacent bunch crossings overlap in the detector. It became particularly important to minimize the effects of pileup in 2012, when $<\mu>=20.7$. An event display illustrating the challenge posed by pileup in the detector is shown in Figure 3.6. 


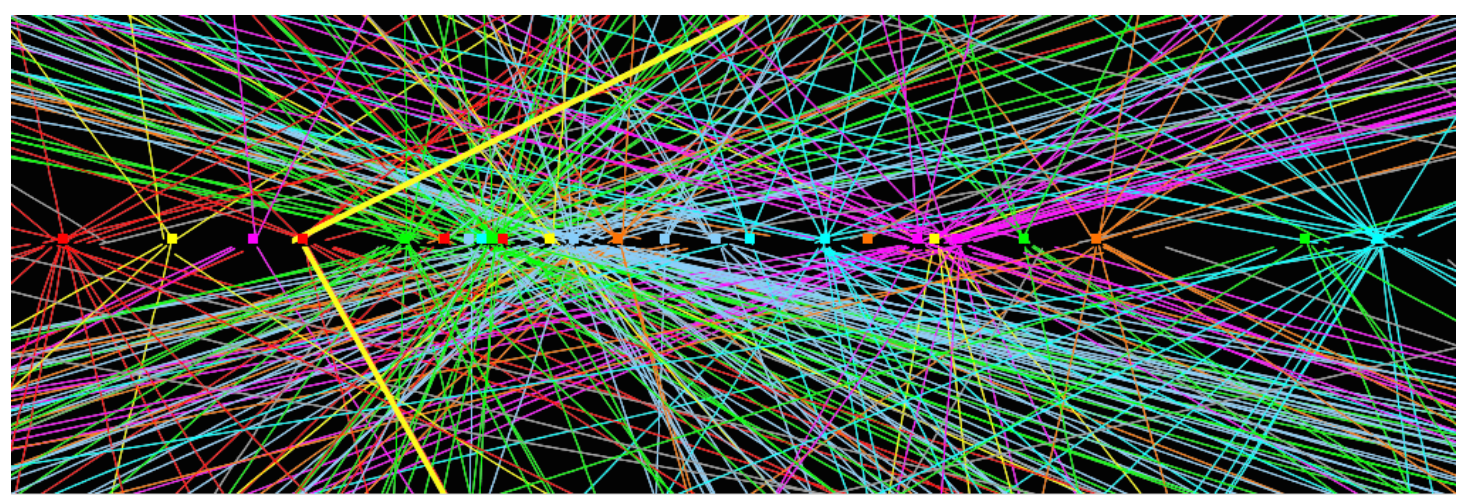

FiguRE 3.6: An example of a $Z \rightarrow \mu \mu$ event featuring high pileup in ATLAS, recorded in 2012 at $\sqrt{s}=8 \mathrm{TeV}$. The two reconstructed muon tracks are depicted by yellow lines. In total, 20 primary vertices were reconstructed [37].

\subsubsection{The ATLAS coordinate system}

The detector is nominally forward-backward symmetric about the interaction point, which is located at the centre of the detector; this point defines the origin of the coordinate system. A simplified schematic of the detector, illustrating this coordinate system, is shown in Figure 3.7. The positive $x$-axis points inwards from the interaction point to the centre of the LHC ring and the positive $y$-axis points upwards. The $z$-axis lies in the direction of the beam line; the side of the detector with $z<0$ is known as side $\mathrm{C}$ and the side having $z>0$ is known as side A. The azimuthal angle, $\phi$, is measured about the $z$-axis, while the polar angle, $\theta$, is measured from the $z$-axis. Instead of the polar angle, the pseudorapidity $\eta$, defined as

$$
\eta=-\ln \left[\tan \left(\frac{\theta}{2}\right)\right]
$$

is often used. For reference, $\eta=0$ points in the upward direction, while $\eta=\infty$ points along the direction of the beamline. The detector spans the approximate 
range $|\eta|<5$. The distance in $\eta-\phi$ space, $\Delta R$, is given by

$$
\Delta R=\sqrt{\Delta \eta^{2}+\Delta \phi^{2}}
$$

The transverse momentum $\left(p_{T}\right)$, transverse energy $\left(E_{T}\right)$, and the missing transverse momentum (more commonly known as missing transverse energy, $E_{\mathrm{T}}^{\text {miss }}$, since most of its components are measured in the calorimeter) are measured in the $x-y$ plane, which lies transverse to the direction of the beams.

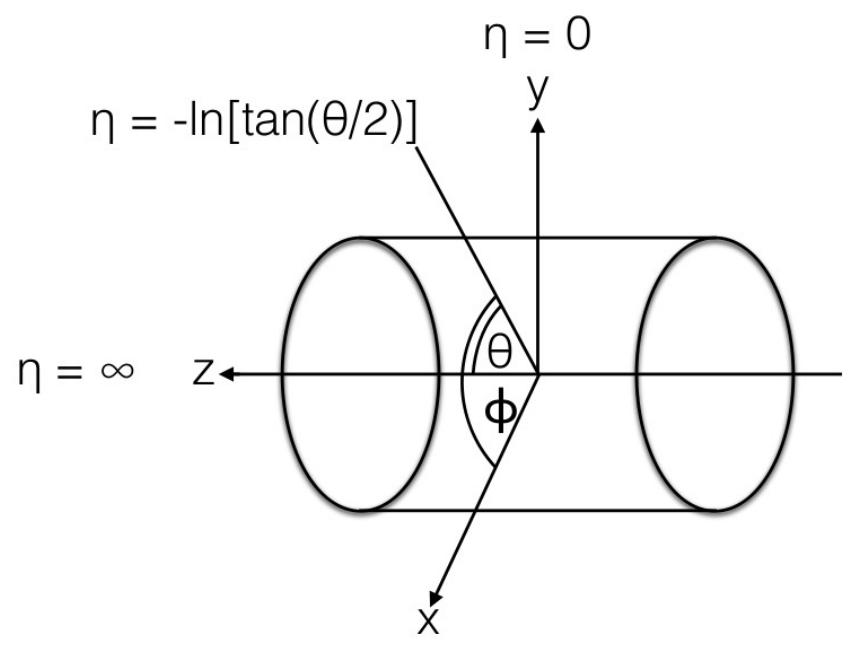

Figure 3.7: A simplified schematic of the ATLAS detector, illustrating the coordinate system used in the experiment.

\subsubsection{Inner detector}

The inner detector (ID) [30, 38, 39], pictured in Figure 3.8, encompasses three subdetectors tasked with measuring the position and momenta of charged particles in the region $|\eta|<2.5$ : the pixel detector [40, 41], the semiconducter tracker (SCT) [42-44], and the transition radiation tracker (TRT) [45, 46]. The inner 
detector is situated inside a $2 \mathrm{~T}$ solenoidal magnetic field, which bends the trajectories of charged particles, thus providing charge identification capabilities. Figure 3.9 shows the material layers traversed by a particle passing through the inner detector.

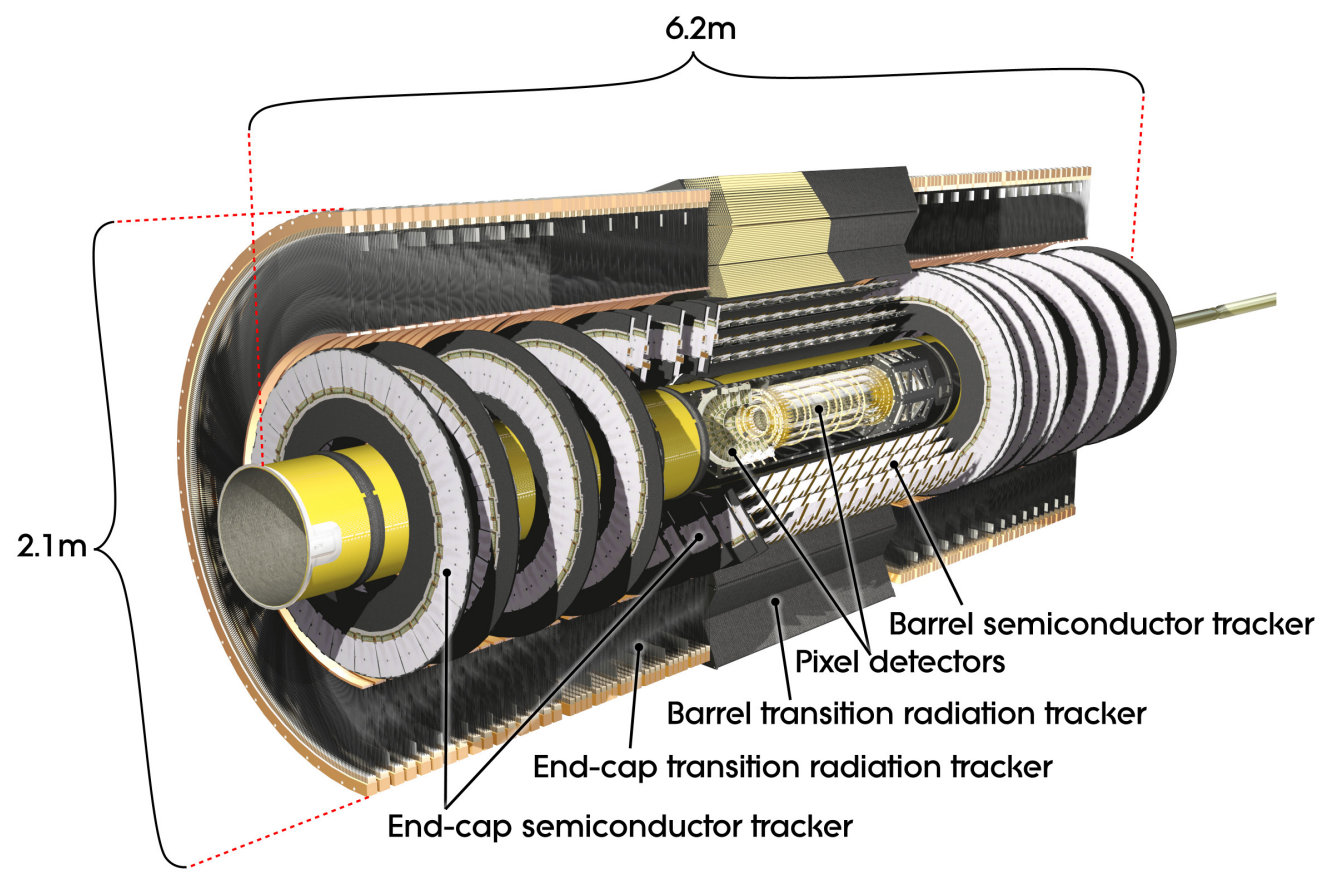

Figure 3.8: The ATLAS inner detector [30].

\subsubsection{Pixel detector}

Located closest to the beam pipe, the silicon pixel detector comprises 80 million channels distributed across three layers, and is intended to perform pattern recognition for the purpose of reconstructing charged particle tracks. It provides crucial primary vertex reconstruction capabilities necessitated by high pileup conditions at the design luminosity of the LHC, and is also critical for identifying secondary vertices for the purpose of $b$-jet tagging. The innermost pixel layer, known as the 
B-layer, is situated a mere $5 \mathrm{~cm}$ from the beam line and plays an important role in distinguishing electrons from converted photons.

\subsubsection{Semiconductor tracker}

The SCT is the second component of the inner detector; it consists of four double layers of silicon microstrips in the barrel and nine disks in each end-cap, yielding an additional eight hits per charged particle track, and includes over six million readout channels. It provides an important contribution to the high-precision vertex position, impact parameter, and momentum measurements.

\subsubsection{Transition radiation tracker}

The TRT is made up of approximately 350,000 polyimide straw drift tubes arranged parallel to the beam pipe in the barrel and radially in the end-caps. These straws contain a mixture of $\mathrm{Xe}, \mathrm{CO}_{2}$ and $\mathrm{O}_{2}$, which is ionized by charged particles as they pass through the TRT material; the ionization electrons then drift to wire anodes located within the straws, resulting in an electric current. On average, 35 hits per charged particle are measured in the range $|\eta|<2.0$. This large number of hits provides continuous tracking and serves to complement the precision measurements of the pixels and SCT, yielding robust pattern recognition and momentum measurements. The gas mixture also absorbs transition radiation emitted by charged particles, resulting in additional ionization. The amount of transition radiation emitted is specific to each type of charged particle; in particular, electrons emit significantly more transition radiation than hadrons, and so the fraction of hits passing a high electronic threshold can be used to distinguish electrons from hadrons. 


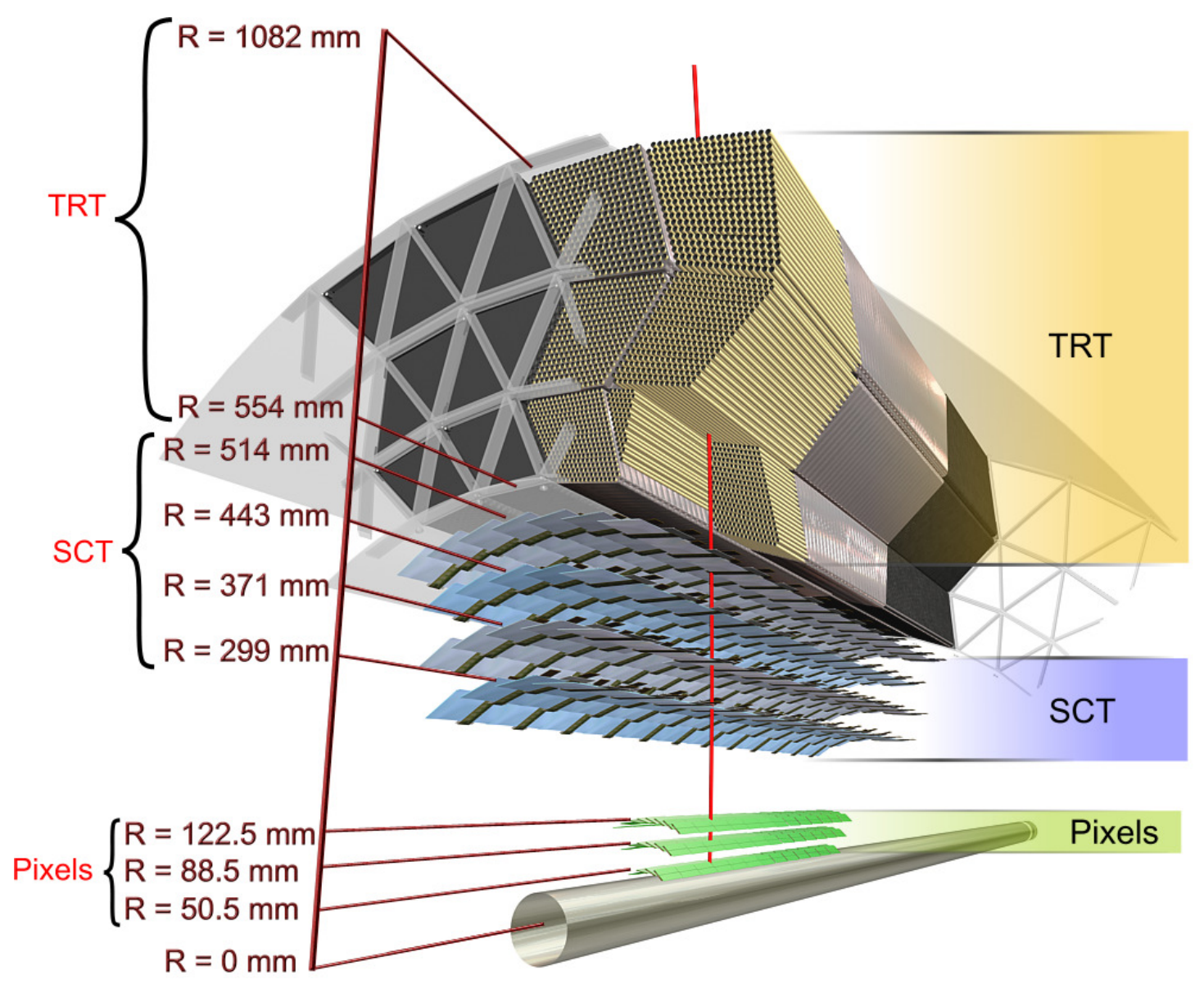

Figure 3.9: The concentric layers of the ATLAS barrel inner detector: the pixel detector, the semiconductor tracker (SCT), and the transition radiation tracker (TRT) [30].

\subsubsection{Calorimeters}

As particles pass through the detector material, they lose energy in the form of electromagnetic or hadronic showers. The amount of material, in radiation lengths, traversed as a function of $|\eta|$ is shown in Figure 3.10. (A radiation length, $X_{0}$, is the mean distance an electron travels in a material before its energy is reduced by a factor of $1 / e$ due to bremsstrahlung, or $7 / 9$ of the mean free path travelled by a photon before it converts to an $e^{+} e^{-}$pair.) ATLAS contains two different types of calorimeters, pictured in Figure 3.11, which surround the inner detector and 
measure the deposited shower energy. In the region $|\eta|<2.5$, the electromagnetic calorimeter is highly granular in order to accurately identify and measure electrons and photons in conjunction with the inner detector, which spans the same range in $|\eta|$. Beyond this limit, up to $|\eta|<4.9$, the granularity is coarser, but still sufficient for jet and missing transverse energy measurements.

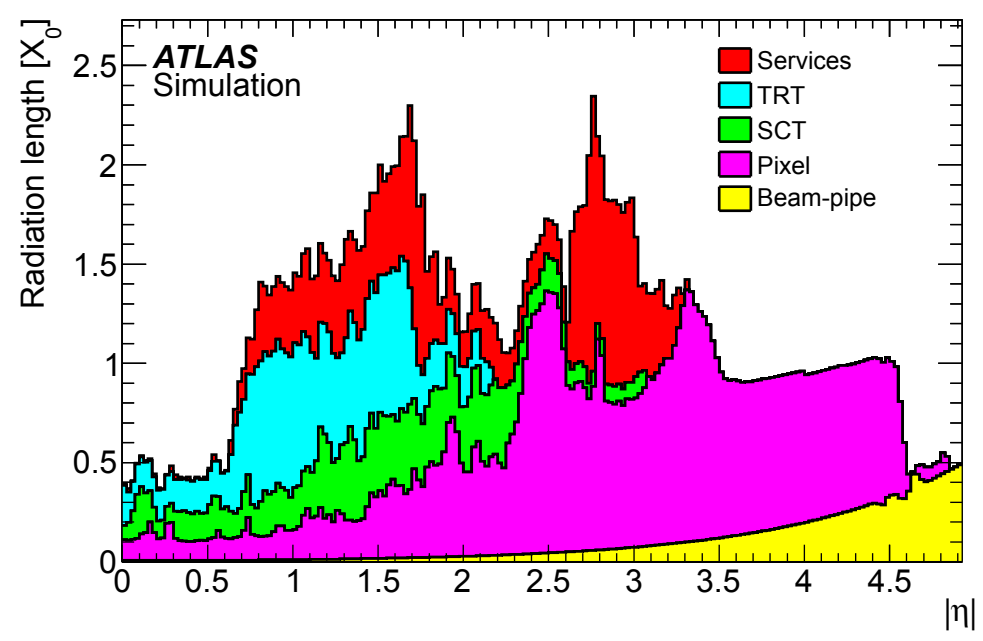

Figure 3.10: Amount of material (in radiation lengths) in front of the cryostat housing the ATLAS electromagnetic calorimeter [47].

\subsubsection{Electromagnetic calorimeter}

The electromagnetic calorimeter [48] measures the energy deposited by electrons and photons, which primarily lose energy in the form of bremsstrahlung and pair production, respectively. Each initial bremsstrahlung photon or electron-positron pair then deposits energy in a similar fashion, triggering a cascade of electromagnetically interacting particles.

The electromagnetic calorimeter is made up of alternating lead absorbers and liquid argon sampling layers arranged in a unique accordion geometry allowing for total symmetry in $\phi$, with a minimum thickness of 22 radiation lengths in order 


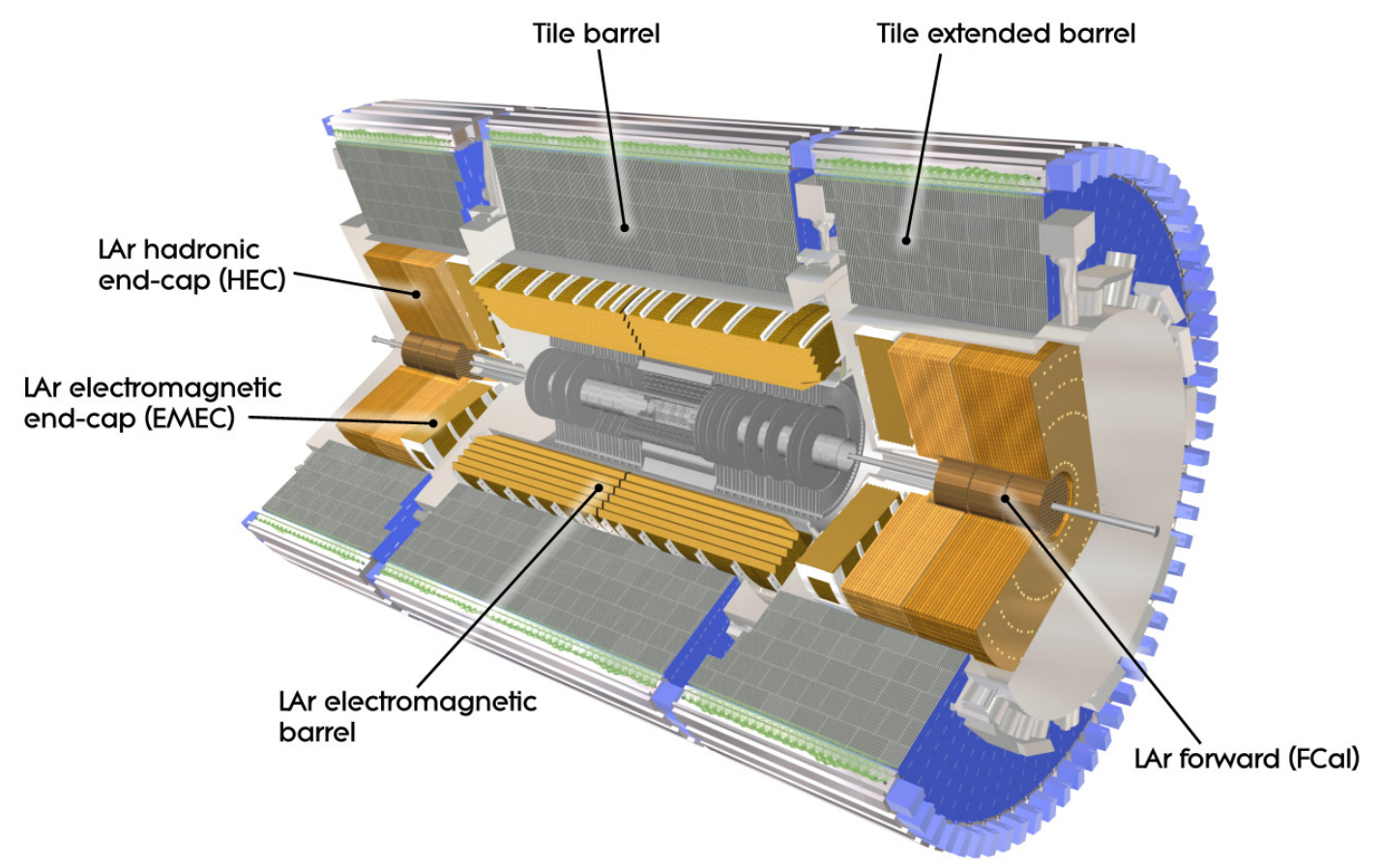

Figure 3.11: The ATLAS calorimeter system, which measures energy deposited during electromagnetic and hadronic showers [30].

to fully contain electromagnetic showers. An electromagnetic calorimeter module is illustrated in Figure 3.12. The calorimeter is divided into a barrel $(|\eta|<1.475)$ and two end-cap sections $(1.375<|\eta|<3.2)$, and is composed of three layers: the first layer consists of finely segmented strips in the $|\eta|$ direction, which provide precise position measurements and help to distinguish between prompt photons and $\pi^{0} \rightarrow \gamma \gamma$ decays, while the second and third layers are made up of progressively coarser cells. A typical electromagnetic shower deposits most of its energy in the second layer. The third layer serves to contain the tails of the electromagnetic showers and provides discriminating power against hadronic showers. In the region $|\eta|<1.8$, a liquid argon presampler measures the energy losses of electrons and photons upstream of the calorimeter. The energy resolution of the electromagnetic barrel calorimeter has been measured to be $\sigma_{E} / E=10 \% \cdot \sqrt{\mathrm{GeV}} / \sqrt{E} \oplus 0.17 \%$, 


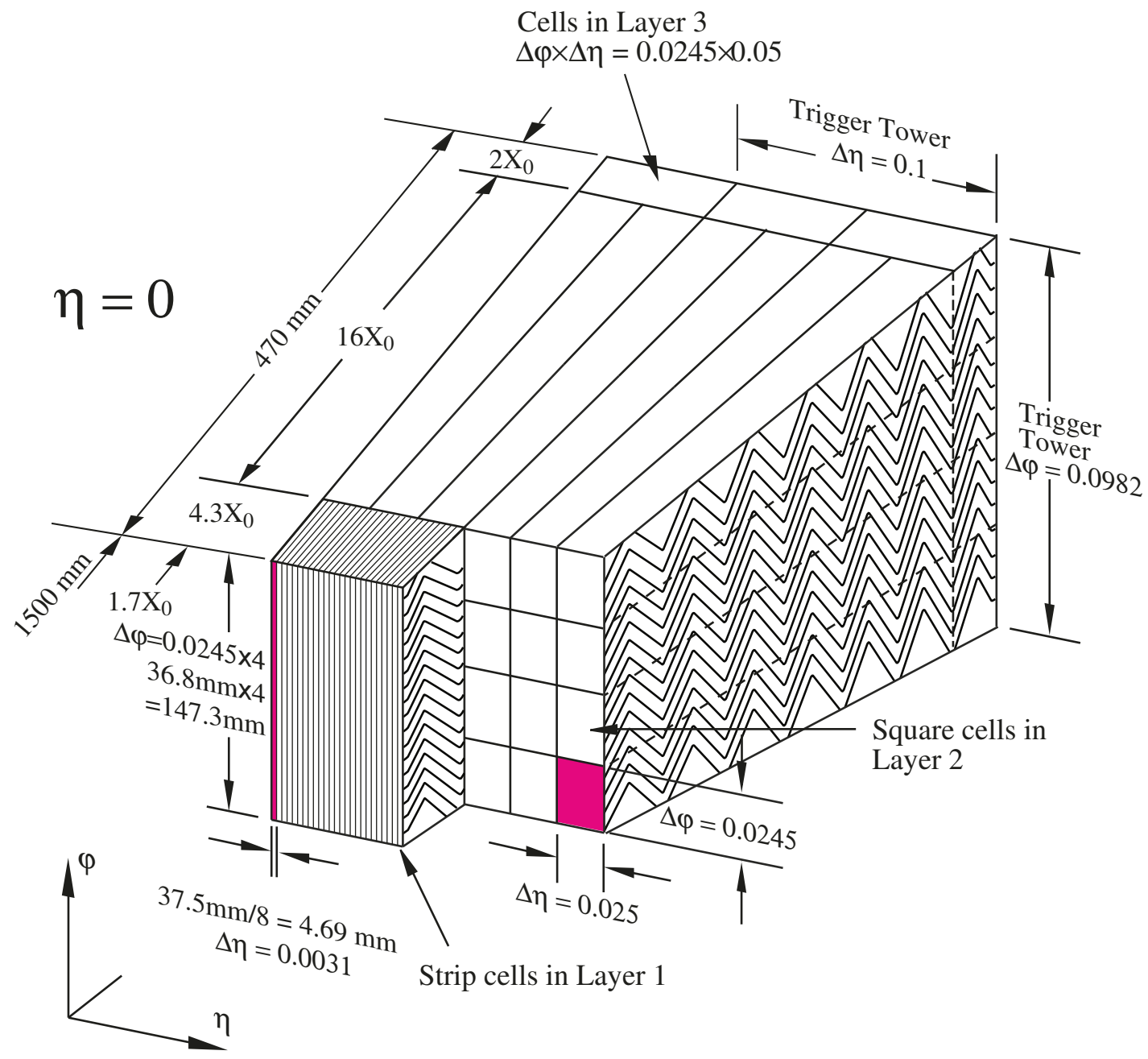

FiguRE 3.12: A barrel module of the electromagnetic calorimeter, illustrating the novel accordion geometry and the granularities of the three sampling layers in $\eta$ and $\phi[30]$.

where $\sigma_{E}$ is the spread of the energy measurements and $E$ is measured in $\mathrm{GeV}$ [30].

\subsubsection{Hadronic calorimeters}

Several calorimeters measure the energy deposited by hadronic showers; these detectors have a minimum thickness of 9.7 interaction lengths (the mean free path travelled by a hadron before undergoing a nuclear interaction) in order to contain the showers. 
In the barrel, hadronic shower energy is sampled by the tile calorimeter [49], consisting of a steel absorber medium and a scintillating tile sampling medium. It covers the region $|\eta|<1.0$; two additional extended barrels provide additional coverage in the region $0.8<|\eta|<1.7$. The measured energy resolution is on the order of $\sigma_{E} / E=56.4 \% \cdot \sqrt{\mathrm{GeV}} / \sqrt{E} \oplus 5.5 \%$, where $E$ is in $\mathrm{GeV}$ [30].

Two liquid argon calorimeter systems are present in the end-caps. The first of these is the hadronic end-cap calorimeter (HEC) [50], which uses copper as an absorber and covers the region $1.5<|\eta|<3.2$. The forward calorimeters (FCal) [51] provide coverage in the forward region, 3.1 $<|\eta|<4.9$. The FCal comprises three separate modules per end-cap; the first module is a copper - liquid argon electromagnetic calorimeter, while the second and third modules are tungsten - liquid argon hadronic calorimeters. The FCal's hadronic energy resolution has been measured to be on the order of $\sigma_{E} / E=70 \% \cdot \sqrt{\mathrm{GeV}} / \sqrt{E} \oplus 3 \%$, where $E$ is in units of $\mathrm{GeV}[30]$.

\subsubsection{Muon Spectrometer}

The muon spectrometer (MS) [52], pictured in Figure 3.13, spans the region $|\eta|<$ 2.7 and measures the momenta of muons, which pass through the inner detector and calorimeter systems. Charge identification is performed by deflecting the muon trajectories with three superconducting air-core toroid magnets; one provides a field of $0.5 \mathrm{~T}$ in the barrel, and two end-cap toroids provide fields of $1 \mathrm{~T}$. The muon chambers are arranged in three concentric cylindrical layers in the barrel and four wheels in the end-caps.

The muon spectrometer comprises four subdetector systems. Precision tracking measurements are performed by the monitored drift tubes (MDT) in the 


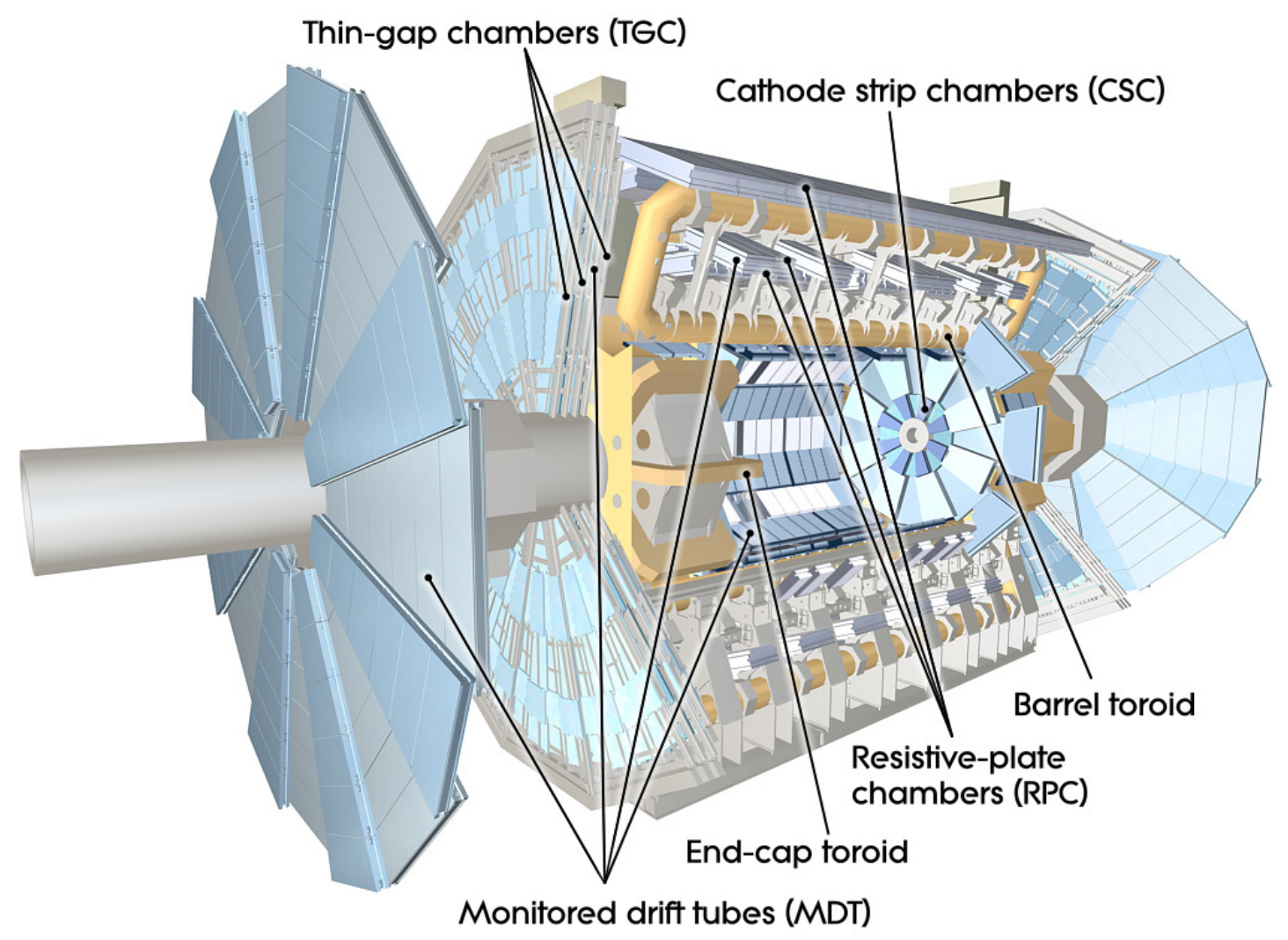

FIGURE 3.13: The ATLAS muon spectrometer [30].

region $|\eta|<2.7$. In the innermost layer, this task is instead performed by cathode strip chambers (CSC) in the region $2.0<|\eta|<2.7$. The resistive plate chambers (RPC), which cover the region $|\eta|<1.05$, and the thin gap chambers (TGC), which cover the region $1.05<|\eta|<2.4$, provide muon triggering capabilities.

\subsubsection{Trigger}

The high collision rate and large production cross-sections at the LHC necessitate the use of a trigger system in order to efficiently maximize the number of interesting physics events recorded for analysis purposes, while filtering out uninteresting events from inelastic collisions. At the LHC's design luminosity and bunch spacing, 
collisions occur at a rate of about $1 \mathrm{GHz}$, while ATLAS is only capable of recording events at a rate of $400 \mathrm{~Hz}$, due to data processing and storage constraints. The ATLAS trigger and data acquisition system (TDAQ) is a multi-step hardwareand software-based system that filters events based on progressively more detailed detector information.

Events detected by ATLAS must first pass the level-1 (L1) trigger [53]. This hardware-based trigger makes decisions using basic information from the subdetectors and quickly sends its results to the front-end electronics. At this stage, the event acceptance rate is sharply reduced to $\sim 75 \mathrm{kHz}$.

Information about regions of interest (RoIs) in which EM cluster, muon, $\tau$, jet, and $E_{\mathrm{T}}^{\mathrm{miss}}$ objects, which are associated with interesting physics processes, have been found is then sent on as input to the second step of the trigger system: the level-2 (L2) trigger [54]. In addition to using more refined information from the full granularity of the calorimeters and muon spectrometer, the L2 trigger, which is software-based, also exploits the information from the inner detector to accept events. The L2 trigger further reduces the event rate to $3.5 \mathrm{kHz}$.

The final step in the trigger system is the event filter (EF) [54]. This trigger is a processing farm, which uses offline analysis algorithms to select interesting events and separate them into physics analysis streams, depending on the physics objects found in the event. These streams include: electrons, muons, jets, photons, $E_{\mathrm{T}}^{\text {miss }}, \tau$-leptons, and $B$-physics. The event filter and the L2 trigger are collectively known as the high-level trigger (HLT). Events passing the HLT are then recorded at a rate of about $400 \mathrm{~Hz}$ to be used for physics analysis. 


\subsection{Physics object reconstruction}

The process of taking the raw data collected by ATLAS in the form of position and energy measurements and associating these measurements with particular types of particles is known as reconstruction. The ATLAS reconstruction software uses information about particle trajectories and momenta from the tracking and muon detectors and energy deposited in the calorimeters (or the absence of these quantities) in order to build a "physics object" corresponding to a particle, such as an electron or muon. A graphical explanation of the detector information used to reconstruct various physics objects is shown in Figure 3.14.

\subsubsection{Electron reconstruction}

Electrons are charged particles, and so they produce tracks in the inner detector. In addition, they deposit energy in the electromagnetic calorimeter, as shown in Figure 3.14. The ATLAS electron reconstruction algorithms [56] use the information from these subdetectors to build tracks and calorimeter clusters; when the trajectory of a track in the inner detector is found to match the position of a cluster in the calorimeter, the result is a reconstructed electron candidate.

The principal algorithm for reconstructing electrons is seeded by a cluster in the electromagnetic calorimeter. Clusters are built using a sliding window algorithm [57], which sums the contributions of cells in a rectangular window of fixed size: $N_{\eta} \times N_{\phi}=3 \times 5(5 \times 5)$ cells in the barrel (end-caps), where a cell measures $\Delta \eta \times \Delta \phi=0.025 \times 0.025$ in the second layer of the calorimeter (refer to Figure 3.14). The position of the window is chosen such that the transverse energy contained within is a local maximum and exceeds $2.5 \mathrm{GeV}$. 


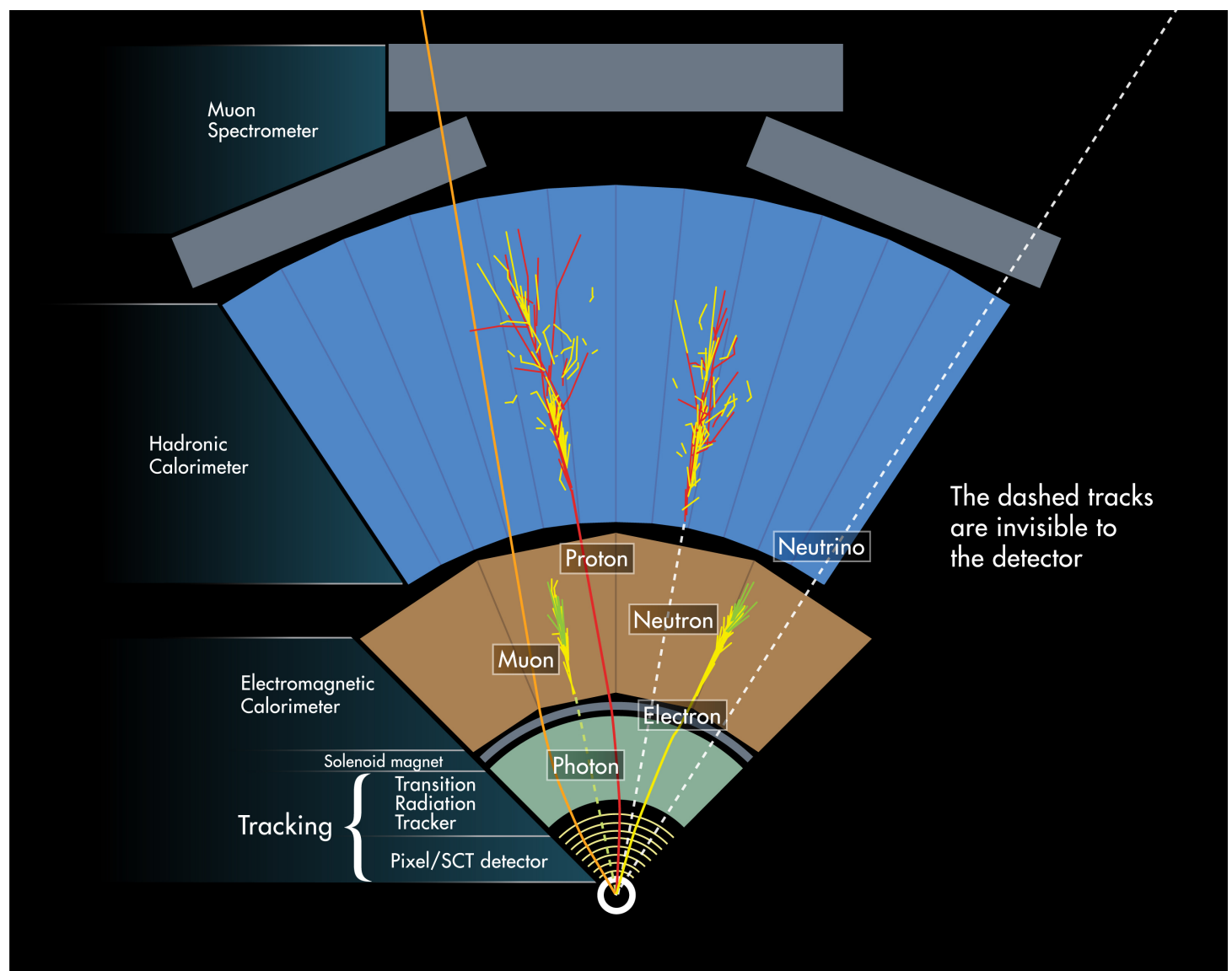

Figure 3.14: Physics object reconstruction in ATLAS [55]. Information from the various subdetector systems is used to determine the type of particle detected and derive its properties.

Once seed clusters have been found, the next step, known as track matching, is performed. For clusters in the range $|\eta|<2.5$, i.e. the range spanned by the inner detector, reconstructed tracks are extrapolated to their last measurement point in the ID to the second layer of the electromagnetic calorimeter. The track matching is fairly loose at this stage; the distance between the track impact point and the position of the cluster must agree within $\Delta \eta<0.05$. In the $\phi$ plane, the track is required to pass within $\Delta \phi<0.1$ in the track's direction of curvature and $\Delta \phi<0.05$ on the other side; this difference is to allow for radiative losses due to bremsstrahlung as the electron traverses the detector. In the event of multiple 
tracks matching a cluster, the track with the smallest value of $\Delta R=\sqrt{\Delta \eta^{2}+\Delta \phi^{2}}$ is used.

Upon matching a cluster to a track, the cluster is then rebuilt using towers of $3 \times 7(5 \times 5)$ cells in the barrel (end-caps). The choice of window size reflects the detector geometry: the cells are larger in the barrel, and the magnetic field bends electrons' tracks in the azimuthal plane, requiring additional cells in $\phi$ in order to contain the total shower energy. The cluster energy is computed by taking the sum of the energy deposited in the calorimeter itself, as well as the estimated energy deposited in front of the electromagnetic calorimeter, outside the cluster (lateral leakage), and beyond the electromagnetic calorimeter (longitudinal leakage). The four-momentum of the reconstructed electron is then computed using the cluster energy, with $\eta$ and $\phi$ measured at the vertex of the track.

For the earliest data taken in 2010 and 2011, the same track-finding algorithm, based on a pion hypothesis, was used for all charged particles. This posed problems for the electron reconstruction, since electrons lose significant fractions (on the order of 20-50\%) of their energy due to bremsstrahlung radiation as they pass through the inner detector material. The pion hypothesis did not take these losses into account, and hence could result in underestimations of the momentum at the primary vertex, as well as uncertainties in track matching and charge identification. These issues were particularly significant for low- $p_{T}$ electrons, leading to efficiency losses for analyses involving these particles, notably the $H \rightarrow Z Z^{*} \rightarrow 4 \ell$ analysis.

A solution to this problem came in the form of the Gaussian Sum Filter (GSF) algorithm [58], which is a non-linear generalization of the Kalman filter $[59,60]$. It models the non-Gaussian noise as a weighted sum of Gaussian 
components, each of which contribute to the approximation of the Bethe-Heitler model of bremsstrahlung [61] in order to take radiative losses into account.

The GSF algorithm significantly improves measurements in the bending plane and reduces their dependence on the position in $\eta$ (i.e. on the amount of material traversed). In particular, the measurement of the transverse impact parameter significance $\left(d_{0} / \sigma_{d_{0}}\right.$, where $d_{0}$ is the transverse distance of closest approach to the primary vertex and $\sigma_{d_{0}}$ is the uncertainty on the measurement) is improved. This quantity plays an important role in distinguishing between reconstructed prompt electrons coming from the primary vertex and leptonic decays of heavy quarks, where the final-state leptons originate from displaced vertices. This permits the separation of the signal from the background in analyses involving electrons in the final state. In addition, the electron four-momentum is computed using the refitted track parameters, which results in improved determination of the invariant masses of particles decaying to electrons, such as the $Z$ boson.

For physics analysis purposes, reconstructed electron candidates must also satisfy offline identification criteria, which select candidates based on track quality and EM shower characteristics. There are several operating points, geared towards the demands of particular analyses, which will be discussed in detail in Chapter 4.

\subsubsection{Photon reconstruction}

Like electrons, photons also interact electromagnetically, depositing energy in the EM calorimeter. However, unlike electrons, photons are electrically neutral and so they do not leave tracks in the inner detector, as seen in Figure 3.14 (unless they happen to convert in the detector material). The photon reconstruction 
algorithm $[62,63]$ searches for photons in both the converted (i.e. after undergoing pair production) and unconverted final states.

The clustering algorithm for photons is almost identical to the electron algorithm. It uses sliding windows of size $3 \times 7(5 \times 5)$ cells in the barrel (end-caps) for converted photons. Showers initiated by unconverted photons are narrower, due to the lack of radiation, and so they use a window of size $3 \times 5(5 \times 5)$ cells in the barrel (end-caps).

An unconverted photon is said to be reconstructed if a calorimeter cluster is found without a matching track in the inner detector. Converted photons require a cluster with at least one matched track associated to a conversion vertex. The particular case of a conversion having only one matched track can occur if the conversion occurs late in the photon's trajectory, as it traverses the TRT; the resulting electron and positron are generally still quite close together as they exit the inner detector and the TRT is unable to resolve the two tracks separately.

As for electrons, additional offline selection criteria are applied to photons; the specific requirements vary depending on the nature of the analysis.

\subsubsection{Muon reconstruction}

Muons are heavy, charged leptons which leave tracks in the inner detector and pass through the calorimeters virtually unhindered. They are the only particles to leave signatures in the muon spectrometer, since electromagnetic and hadronic showers are well-contained by the calorimeters. The muon reconstruction algorithms $[64,65]$ identify regions of activity in the muon spectrometer using information from the RPCs and TGCs. Local track segments in individual chambers are first reconstructed in the regions of interest; these segments are then combined 
to form track candidates. Individual hit information is then used to perform a global fit of the track candidates.

Muon candidates are identified as belonging to four distinct categories:

- Stand-alone (SA) These muon candidates have a track reconstructed only in the muon spectrometer, without a matching track in the inner detector. The trajectory is extrapolated back from the muon spectrometer to the point of closest approach to the beam line, while taking into account energy lost in the calorimeters.

- Combined (CB) A track is reconstructed in both the inner detector and the muon spectrometer; these tracks are then combined to form a single muon trajectory.

- Segment-tagged (ST) A track in the inner detector is associated to at least one track segment in the MDTs or CSCs.

- Calorimeter-tagged (CaloTag) In this case, a track in the inner detector is matched to a calorimeter energy deposit corresponding to a minimum ionizing particle. These muons are only identified in the region $|\eta|<0.1$, where the muon spectrometer lacks instrumentation due to the presence of inner detector and calorimeter services.

Two muon reconstruction algorithms are used in ATLAS. The first, known as "STACO," uses the covariance matrices of the measurements of SA and ID muon tracks to perform statistical combinations of those parameters. The second, "MUID," relies on the hits in the ID and MS to perform global refits of muon tracks. For this analysis, the STACO algorithm is used. 


\subsubsection{Jet reconstruction}

Jets are collections of particles produced via the hadronization of quarks or gluons produced in $p p$ collisions. In ATLAS, jets are reconstructed with the anti- $k_{\mathrm{T}}$ algorithm [66] with distance parameter $R=0.4$ or 0.6 . In the calorimeters, jets are constructed using topological clusters [57], seeded by a cell exceeding a given signal-to-noise ratio threshold. Neighbouring cells are added iteratively if they exceed a secondary signal-to-noise ratio threshold; this threshold is lower than that of the original seed cell in order to avoid rejecting the edges of the jet.

Jets, especially those originating from semi-leptonic decays of heavy quarks, are a significant source of background in analyses involving final-state electrons. Methods for distinguishing true electrons from these jets will be discussed in more detail in subsequent chapters.

\subsubsection{Missing transverse energy reconstruction}

As shown in Figure 3.14, neutrinos, which are electrically neutral and almost massless, do not interact at all with the detector; i.e. they leave no tracks and their energy cannot be measured directly. In order to infer the presence of neutrinos (or perhaps other, more exotic, weakly-interacting particles), it is necessary to reconstruct the missing transverse energy, $E_{\mathrm{T}}^{\mathrm{miss}}[67]$. Since the initial momentum in the transverse plane is zero, the $E_{\mathrm{T}}^{\text {miss }}$ of an event can be reconstructed by summing the energies of the calorimeter clusters and momenta of any muons present in the transverse plane; a vector sum not equal to zero corresponds to the $E_{\mathrm{T}}^{\mathrm{miss}}$. 


\subsection{ATLAS Monte Carlo simulation}

In order to model the detector's response to physics processes, a detailed simulation framework has been developed [68]. The ATLAS simulation framework, which relies on the GEANT4 simulation toolkit $[69,70]$, is integrated into the ATHENA computing framework [71].

The simulation chain has several steps. First, at the event generation stage, generator packages are used to simulate proton-proton collisions, and the resulting parton showers and subsequent hadronization of quarks and gluons are modelled according to theoretical calculations (the specific generators used for this analysis are described in Section 5.1 and Section 5.2). Then, the particles are propagated through the fully-simulated ATLAS detector geometry using GEANT4. The resulting output of the simulated detector interaction is a collection of "hits," or energy deposits with their corresponding position and time information. This information is then digitized; i.e. it is converted to a detector response in the form of voltages or currents in the readout channels, as would be measured experimentally. The simulation chain can be customized to model specific LHC beam parameters and detector noise, and pileup events may also be overlaid in order to accurately simulate experimental operating conditions.

As the understanding of the pileup conditions and detector geometry improves over time, the simulations are reproduced using the most up-to-date configuration. For the $7 \mathrm{TeV}$ analysis, two configurations were produced, based on two versions of the detector geometry: MC11C (old geometry) and MC11D (new geometry). For the $8 \mathrm{TeV}$ analysis, three configurations were produced, based on two versions of the pileup profile and the detector geometry: MC12A (old pileup profile, old geometry), MC12B (new pileup profile, old geometry), and MC12C 
(new pileup profile, new geometry). A list of the samples used for this thesis, including the generators and configurations, is provided in Appendix C.

\subsection{Conclusion}

This chapter has introduced the Large Hadron Collider and the ATLAS detector, where the work described in the remaining chapters of this thesis has been performed. The search for the Higgs boson in the four-lepton decay channel relies on the reconstruction of final-state electrons and muons, based on properties measured using complementary information from the inner detector, calorimeters, and muon spectrometer. Reconstructed electrons are subject to further offline identification requirements, which formed a significant component of this thesis work, and will be described in detail in the following chapter. 


\title{
4. Offline electron identification in ATLAS
}

The electron is not as simple as it looks.

— William Lawrence Bragg

\begin{abstract}
(5) (2) 3

HE majority of objects reconstructed as electrons in ATLAS are not, in sfact, prompt, isolated electrons, as shown in Figure 4.1. In addition to true electrons, such as those originating from $W$ or $Z$ boson decays, many reconstructed electrons are the products of heavy quark decays (e.g. $b \rightarrow c+$ $\left.e^{-}+\bar{\nu}_{e}\right)$ or photon conversions $\left(\gamma \rightarrow e^{+} e^{-}\right)$. In addition, hadrons, which may also leave tracks in the inner detector and deposit energy in the EM calorimeter, may be reconstructed as electron objects; these are commonly known as "fakes." For physics analyses involving electrons in the final state, especially when searching for such rare processes as the $H \rightarrow Z Z^{(*)} \rightarrow 4 \ell$ decay, it is of the utmost importance to be able to distinguish true electrons from these other background electron-like objects.
\end{abstract}

In ATLAS, this role is fulfilled by offline electron identification menus which exploit differences in the EM shower shapes and inner detector track properties particular to true and background electrons. As its name suggests, the offline menu is applied by the user at the final analysis stage. Several operating points 


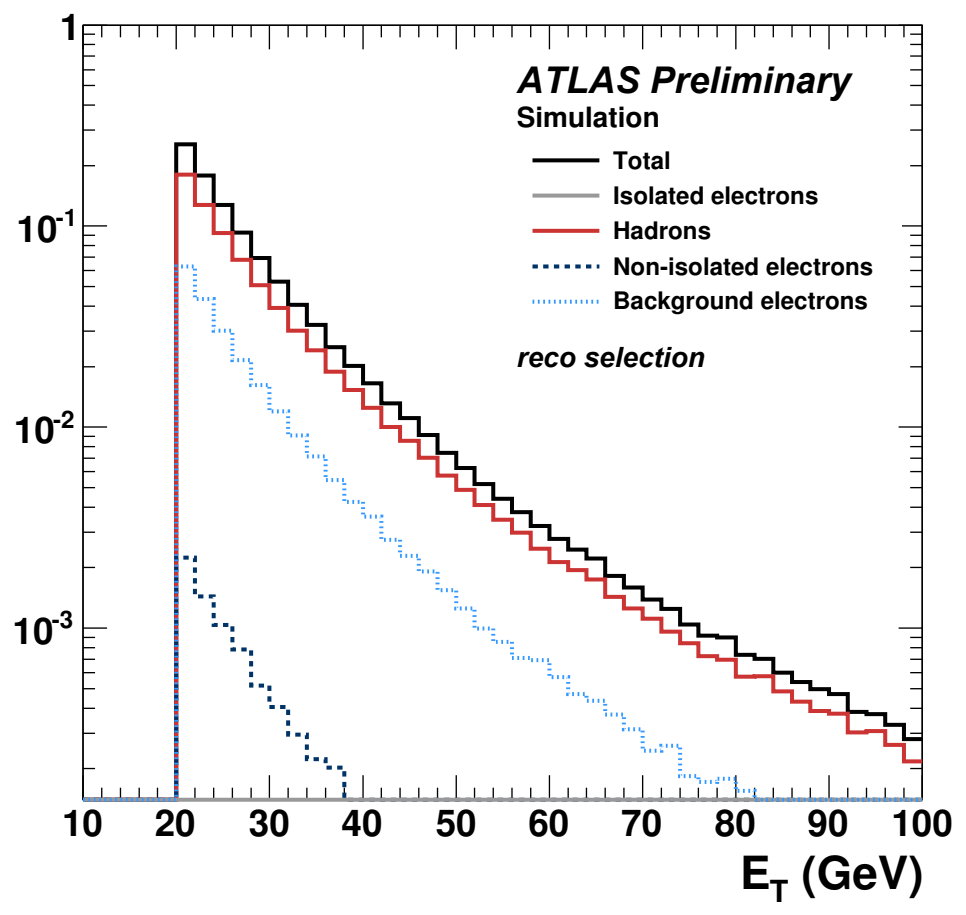

FiguRE 4.1: Distribution of all reconstructed electron objects as a function of cluster $E_{\mathrm{T}}$, separated according to their composition and normalized to the number of candidates. The isolated electron component, which is not visible at this scale, is dominated by hadrons ("fakes"), non-isolated electrons, and background electrons from conversions [72].

exist, each providing a particular level of signal efficiency and background rejection, in order to meet the requirements of the various physics analyses. For example, a search for a rare process, such as the $H \rightarrow Z Z^{(*)} \rightarrow 4 \ell$ search, would typically require an operating point with looser requirements, thus yielding a high efficiency (since four leptons must be identified in total), while an analysis involving precision measurements of a well-understood process, such as $Z \rightarrow e e$ decays, might require a tighter operating point, thus sacrificing efficiency for maximum background rejection. 
Section 4.1 will introduce the discriminating variables used for offline electron identification, and Section 4.2 will describe the standard menus used in ATLAS for the $7 \mathrm{TeV}$ analysis. A special menu, customized to meet the needs of the $H \rightarrow Z Z^{(*)} \rightarrow 4 \ell$ analysis, was designed and employed during the discovery phase of the $8 \mathrm{TeV}$ run $[2,5]$; this menu will be described in Section 4.3. Particular attention will be given in Section 4.3.2.1 and Section 4.3.2.2 to the author's studies of new variables which exploit useful features of the electron reconstruction algorithm and improved background rejection in problematic regions of the detector. As the requirements of the analysis evolved after the discovery, this menu was superceded by a new, multivariate method of electron identification, which will be introduced in Section 4.4.

\subsection{Discriminating variables used in offline elec- tron identification}

As discussed in Chapter 3, electron objects are reconstructed using information from the EM calorimeter and the inner detector. The information from these detectors can also be used to distinguish between true and background electrons. Three broad categories of discriminating variables can be defined: shower shape variables, which describe the amount and distribution of energy deposited in the EM calorimeter; track quality variables, which describe the pattern of charged particles' hits in the inner detector; and track-cluster matching variables, which quantify how well the inner detector track spatially matches the EM calorimeter cluster. 
These variables tend to be sensitive to a number of factors. One such factor is the detector geometry; the amount of material traversed by the particle and the readout granularity of the detectors both vary with $|\eta|$. As the $E_{\mathrm{T}}$ of the electron increases, the distributions of shower shape variables tend to become narrower, which can increase their discriminating power against background. Increased pileup leads to additional noise in the EM calorimeter. These factors should be taken into account when designing an electron identification menu; this is usually done by binning selection cuts in electron $E_{\mathrm{T}}$ and $|\eta|$. Pileup was not a major concern in the $7 \mathrm{TeV}$ analysis, but had a much greater effect on the $8 \mathrm{TeV}$ analysis (recall Figure 3.3). The method of optimizing the electron identification for high-pileup conditions will be discussed in Section 4.3.

In the following pages, shower shape, track quality, and track-cluster matching variables commonly used for electron identification in ATLAS will be defined, and typical distributions of these variables as a function of electron $E_{\mathrm{T}},|\eta|$, and the number of reconstructed primary vertices $\left(N_{\mathrm{vtx}}\right)$ will be presented.

\subsubsection{Shower shape variables}

- $\boldsymbol{E}_{\text {ratio }}$ This corresponds to the relative difference between the first and second energy maxima in the first layer (strips) of the EM calorimeter:

$$
E_{\text {ratio }}=\frac{E_{\max , 1}^{\mathrm{S} 1}-E_{\max , 2}^{\mathrm{S} 1}}{E_{\max , 1}^{\mathrm{S} 1}+E_{\max , 2}^{\mathrm{S} 1}} .
$$

The distribution of this quantity, shown in Figure 4.2, should have a narrow peak at $E_{\text {ratio }}=1$ for true isolated electrons, which tend to result in a single maximum in the EM calorimeter. In the case of non-isolated electrons originating from jets, multiple maxima of similar size are present in the 
calorimeter, resulting in a broader $E_{\text {ratio }}$ distribution. Since there are no strips in the crack region $(1.37<|\eta|<1.52)$ and in the region $2.37<|\eta|<$ 2.47, $E_{\text {ratio }}$ cannot be used as a discriminating variable in those areas. In addition to its sensitivity to $|\eta|, E_{\text {ratio }}$ is also strongly dependent on the cluster $E_{\mathrm{T}}$. It is not significantly affected by pileup conditions, as seen from its lack of dependence on the number of primary vertices reconstructed in the event.

- $\boldsymbol{w}_{\mathrm{s}, \text { tot }}$ The shower width in the first layer of the EM calorimeter is defined as:

$$
w_{\mathrm{s}, \text { tot }}=\frac{\sum_{i} E_{i}\left(i-i_{\max }\right)^{2}}{\sum_{i} E_{i}},
$$

where $i_{\max }$ is the number of the strip containing the largest energy deposit and the summation over $i$ is performed over $\Delta \eta \times \Delta \phi=20 \times 2$ strips. True electrons typically create narrower showers than fakes, as seen in Figure 4.3. As with $E_{\text {ratio }}, w_{\mathrm{s} \text {, tot }}$ is dependent on $E_{\mathrm{T}}$, and also cannot be used in the crack region $(1.37<|\eta|<1.52)$ and in the region $2.37<|\eta|<2.47$. $w_{\mathrm{s} \text {, tot }}$ is not particularly influenced by pileup. Figure 4.3 shows typical distributions of $w_{\mathrm{s}, \text { tot }}$.

- $\boldsymbol{R}_{\boldsymbol{\eta}}$ This variable is defined as the ratio of the shower energy deposited in the second layer of the EM calorimeter in a window of $\Delta \eta \times \Delta \phi=3 \times 7$ cells to that in a window of $\Delta \eta \times \Delta \phi=7 \times 7$ cells.

$$
R_{\eta}=\frac{E_{3 \times 7}^{\mathrm{S} 2}}{E_{7 \times 7}^{\mathrm{S} 2}} .
$$

Since electrons tend to deposit the majority of their energy in a window of $3 \times 7$ cells, this quantity should be distributed around a narrow peak at $R_{\eta}=1$ for electrons, whereas the distribution for background sources should 

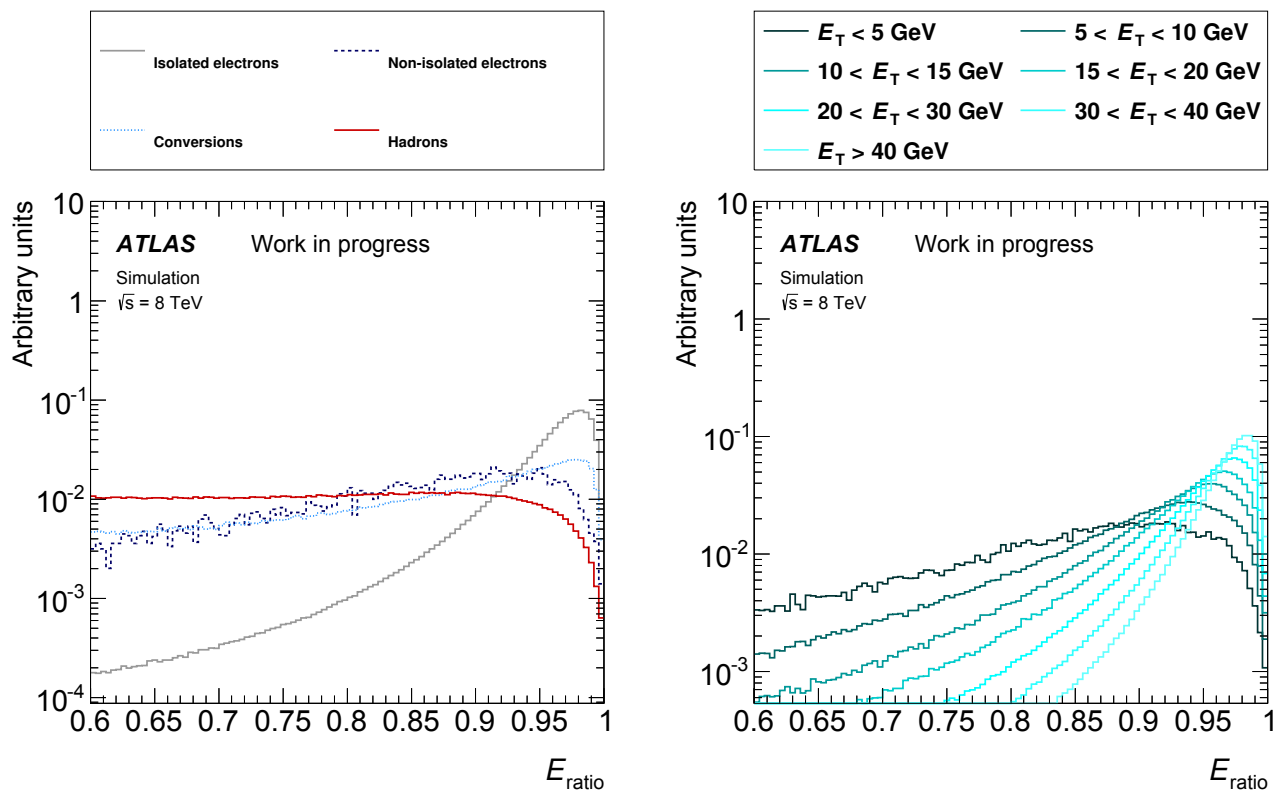

(a) Composition dependence

(b) $E_{\mathrm{T}}$ dependence
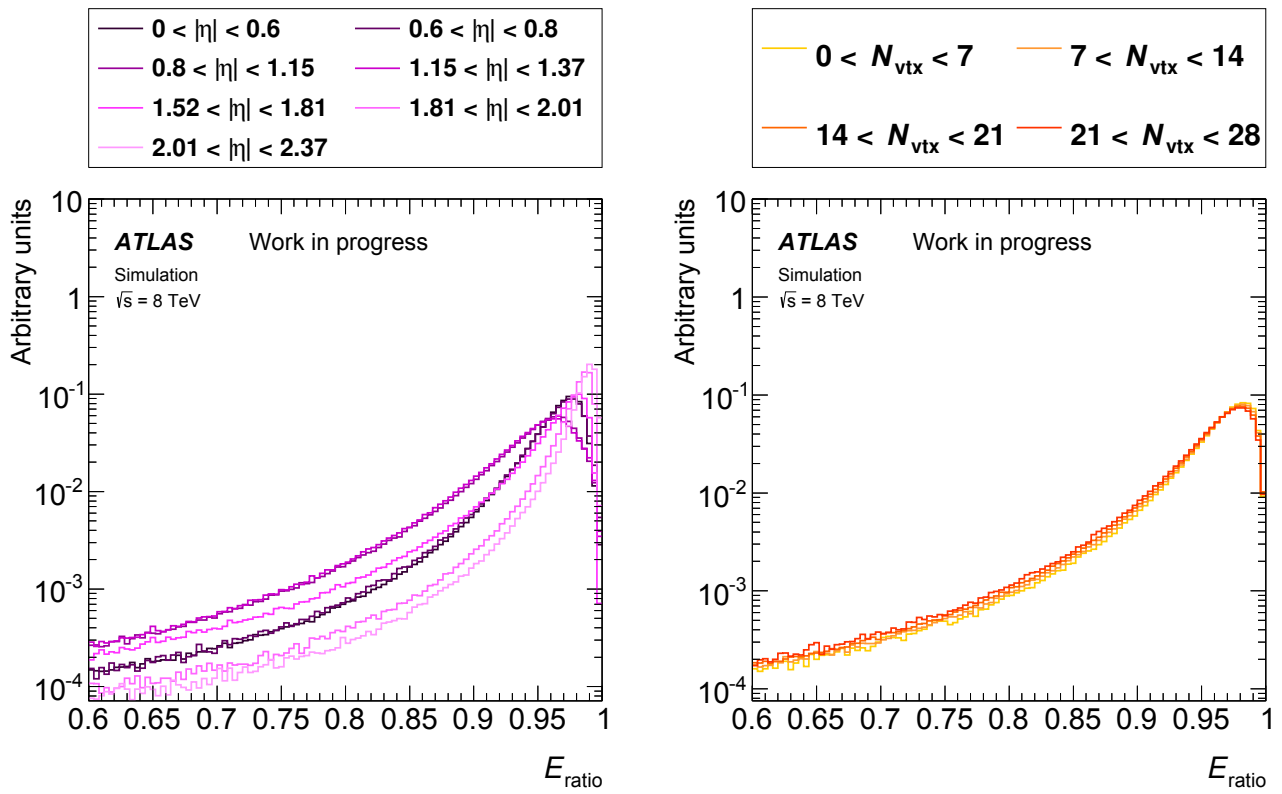

(c) $|\eta|$ dependence

(d) Pileup dependence

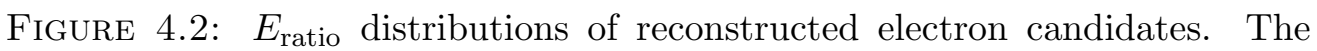
distributions are shown in (a) for isolated electrons, as well as the major background components. Figures (b) - (d) illustrate the dependence of the distributions of isolated electrons on $E_{\mathrm{T}},|\eta|$, and pileup. 

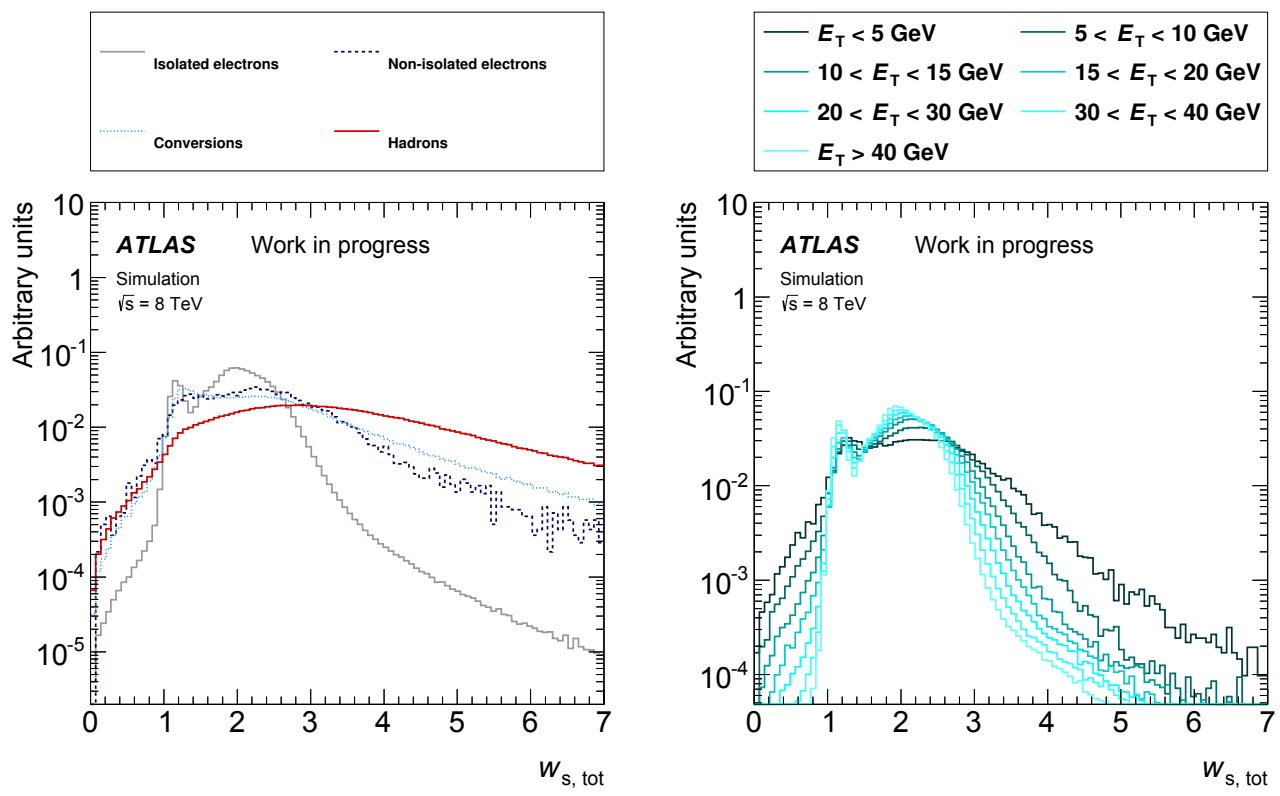

(a) Composition dependence

(b) $E_{\mathrm{T}}$ dependence

$\begin{array}{|cc|}-0<|\eta|<0.6 & 0.6<|\eta|<0.8 \\ 0.8<|\eta|<1.15 & 1.15<|\eta|<1.37 \\ 1.52<|\eta|<1.81 & 1.81<|\eta|<2.01 \\ 2.01<|\eta|<2.37 & \end{array}$

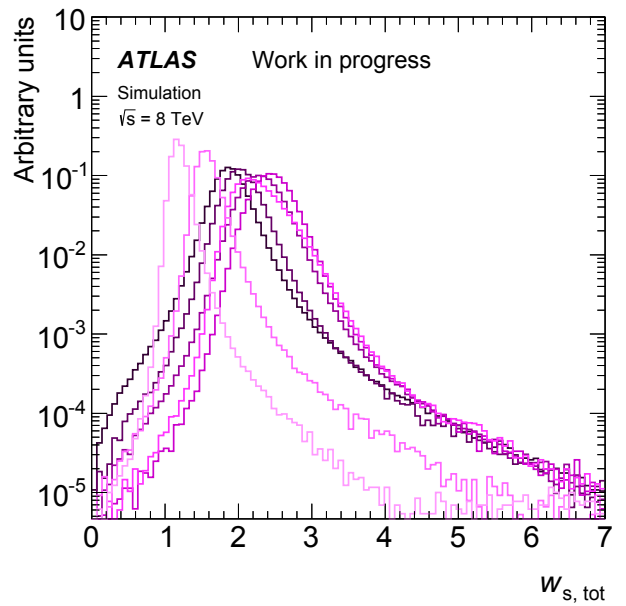

(c) $|\eta|$ dependence
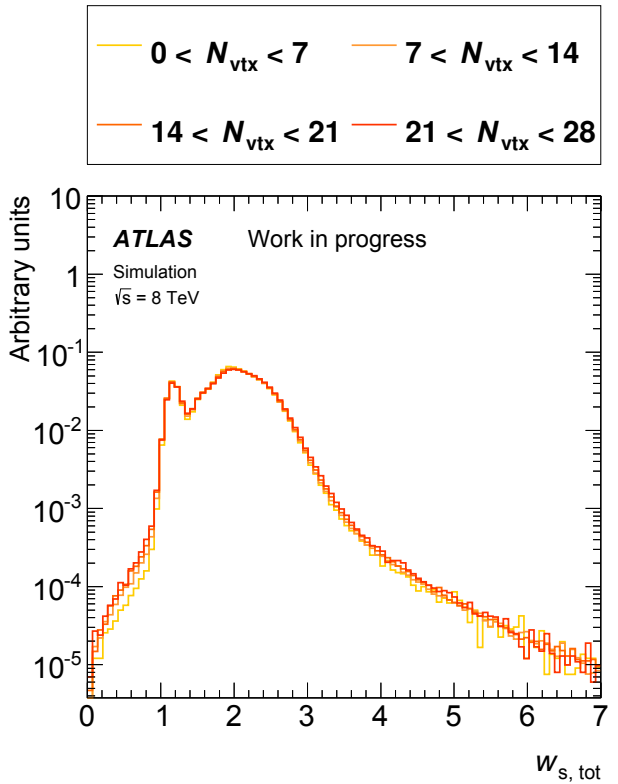

(d) Pileup dependence

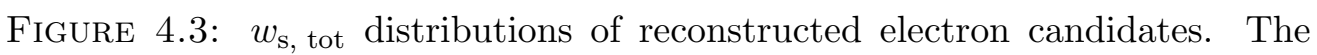
distributions are shown in (a) for isolated electrons, as well as the major background components. Figures (b) - (d) illustrate the dependence of the distributions of isolated electrons on $E_{\mathrm{T}},|\eta|$, and pileup. 
be broader and shifted to somewhat lower values, as seen in Figure 4.4. $R_{\eta}$ tends to be strongly dependent on $E_{\mathrm{T}}$ and $|\eta|$. It is somewhat dependent on pileup, due to the presense of noise in the calorimeter in high pileup conditions.

- $\boldsymbol{w}_{\eta_{2}}$ This refers to the width in the second sampling layer of the EM calorimeter, quantified by the energy-weighted RMS of the cells in that layer.

$$
w_{\eta 2}=\sqrt{\frac{\sum_{i} E_{i} \eta_{i}^{2}}{\sum_{i} E_{i}}-\left(\frac{\sum_{i} E_{i} \eta_{i}}{\sum_{i} E_{i}}\right)^{2}},
$$

where the summation over $i$ cells is performed over a window of $\Delta \eta \times \Delta \phi=$ $3 \times 5$ cells. True electrons produce narrower showers than background electrons or hadrons; this can be seen in Figure 4.5. $w_{\eta 2}$ is sensitive to the cluster $E_{\mathrm{T}}$, as well as $|\eta|$, but it is not strongly affected by pileup.

- $\boldsymbol{f}_{\mathbf{3}}$ This variable is the fraction of transverse energy deposited in the third sampling layer of the EM calorimeter, with respect to the total transverse energy.

$$
f_{3}=\frac{E_{\mathrm{T}}^{\mathrm{S} 3}}{E_{\mathrm{T}}} .
$$

Since most of the shower energy is deposited in the first two layers of the calorimeter for electrons, this quantity should be close to zero. Hadrons, which penetrate further into the calorimeter, deposit a larger fraction of their energy in the third layer, resulting in a broader distribution, as shown in Figure 4.6. Non-isolated electrons have a similarly broad distribution since their clusters are accompanied by other particles, which penetrate further into the calorimeter. $f_{3}$ exhibits a strong dependence on $E_{\mathrm{T}}$ and $|\eta|$, but is not significantly affected by pileup. 

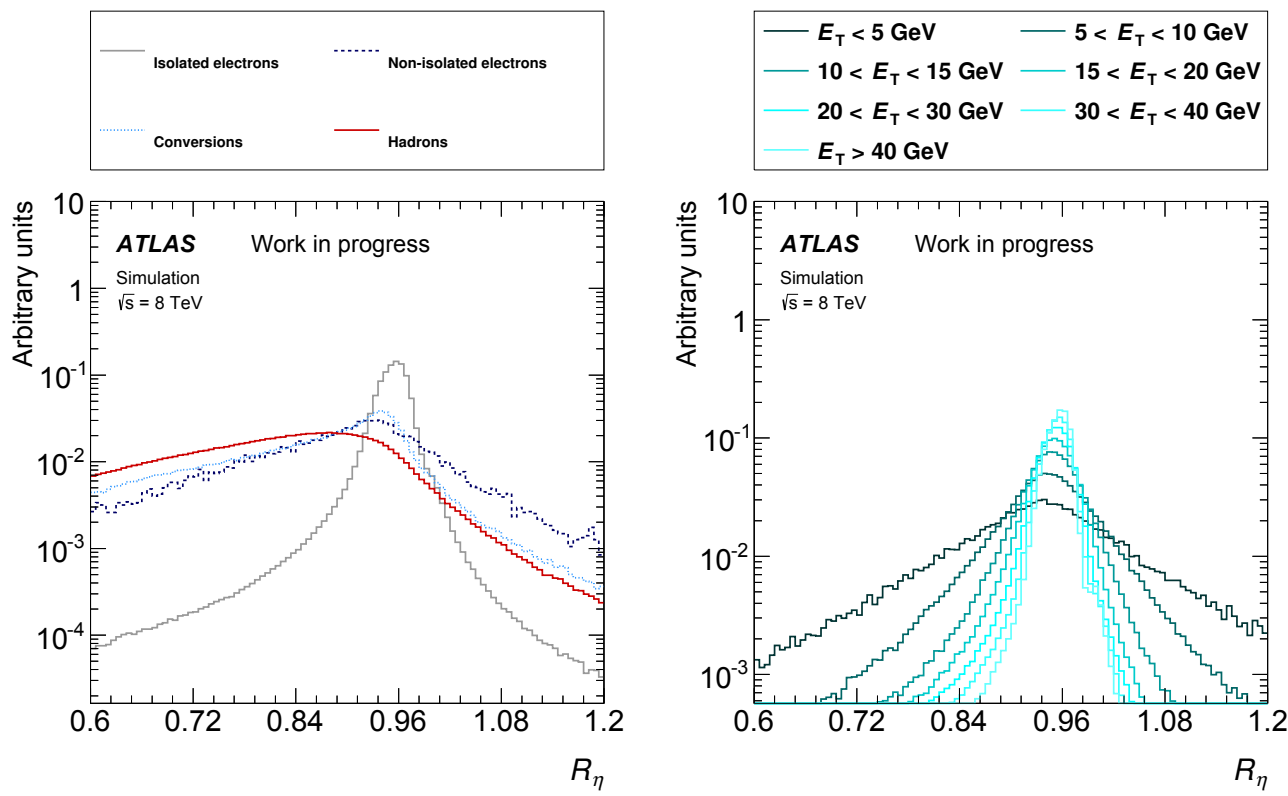

(a) Composition dependence

(b) $E_{\mathrm{T}}$ dependence
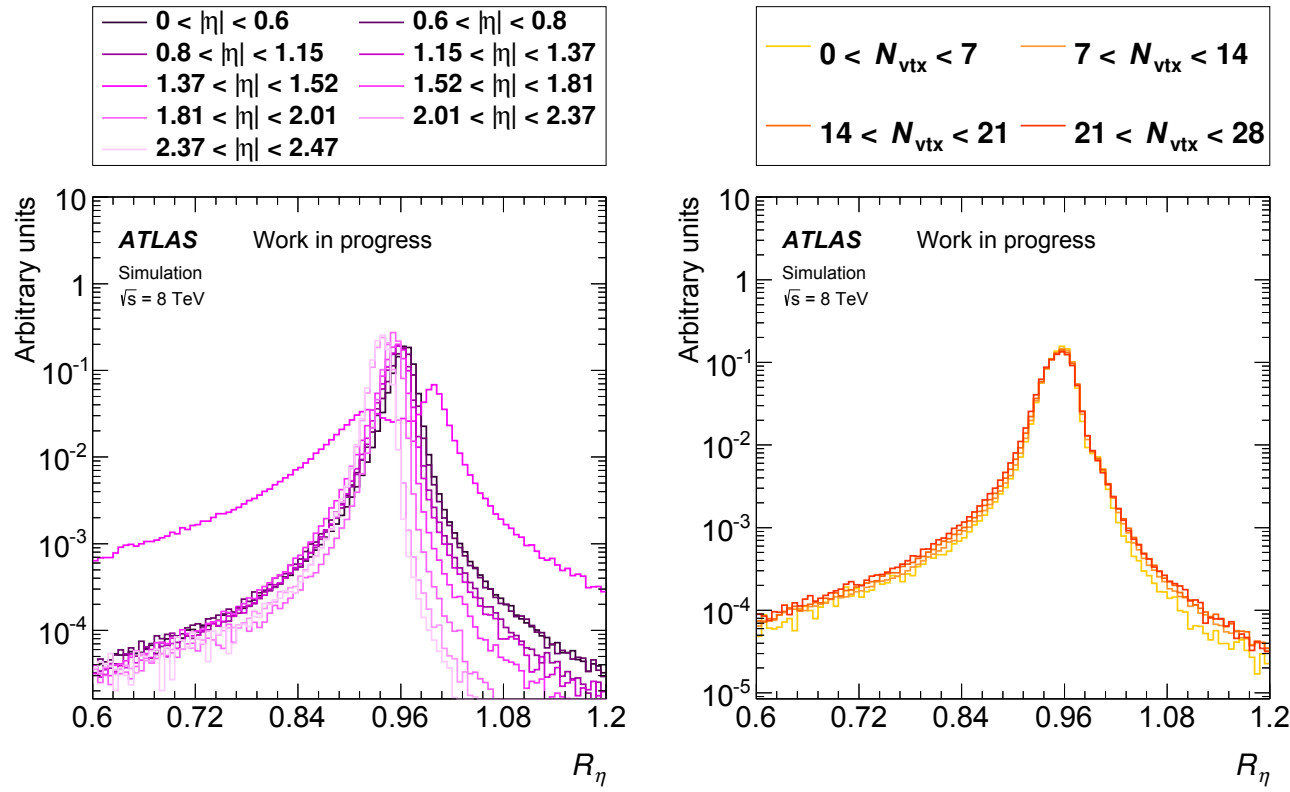

(c) $|\eta|$ dependence

(d) Pileup dependence

FIgURE 4.4: $R_{\eta}$ distributions of reconstructed electron candidates. The distributions are shown in (a) for isolated electrons, as well as the major background components. Figures (b) - (d) illustrate the dependence of the distributions of isolated electrons on $E_{\mathrm{T}},|\eta|$, and pileup. 

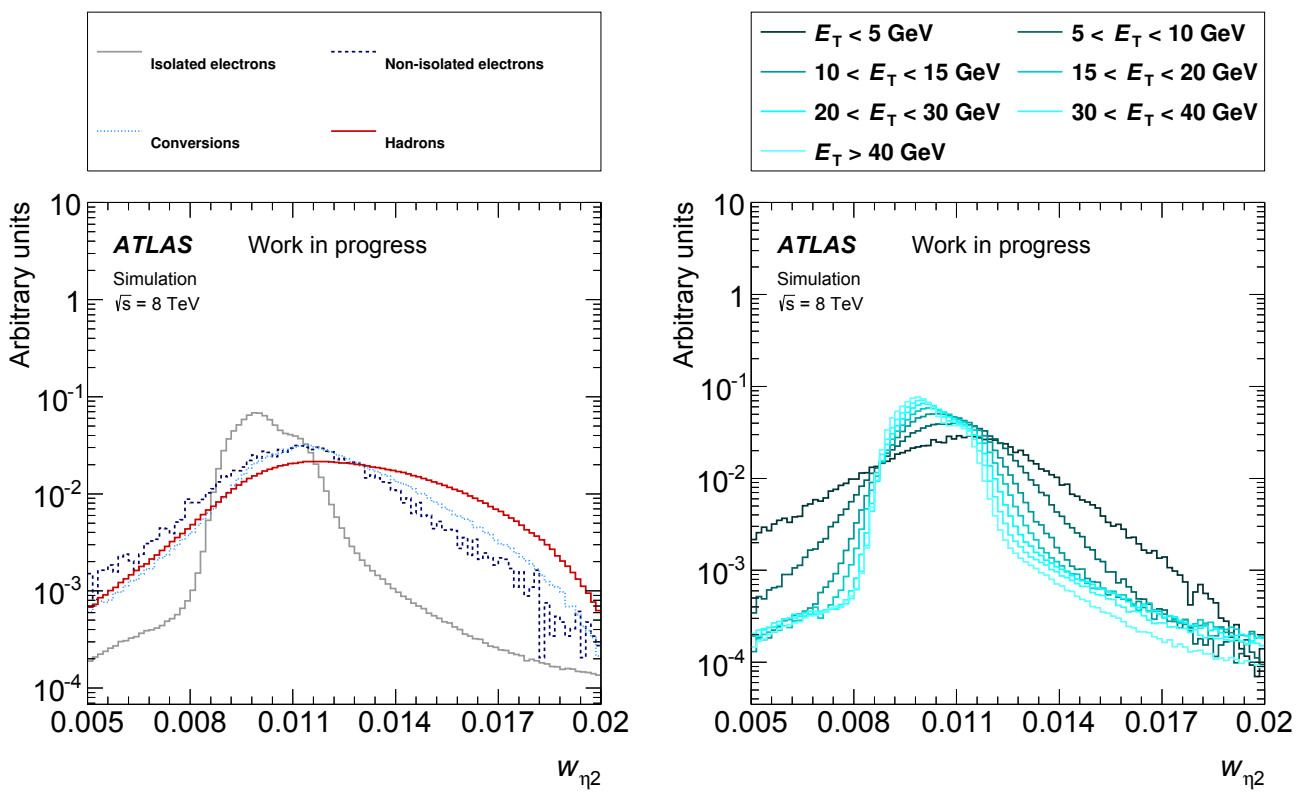

(a) Composition dependence

(b) $E_{\mathrm{T}}$ dependence

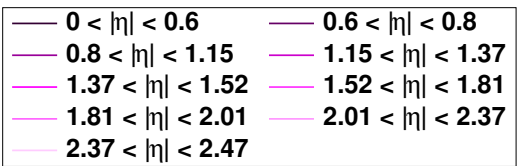

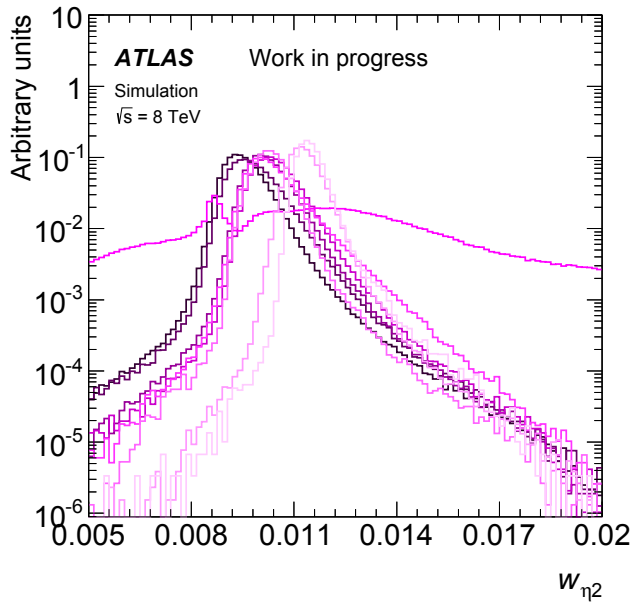

(c) $|\eta|$ dependence
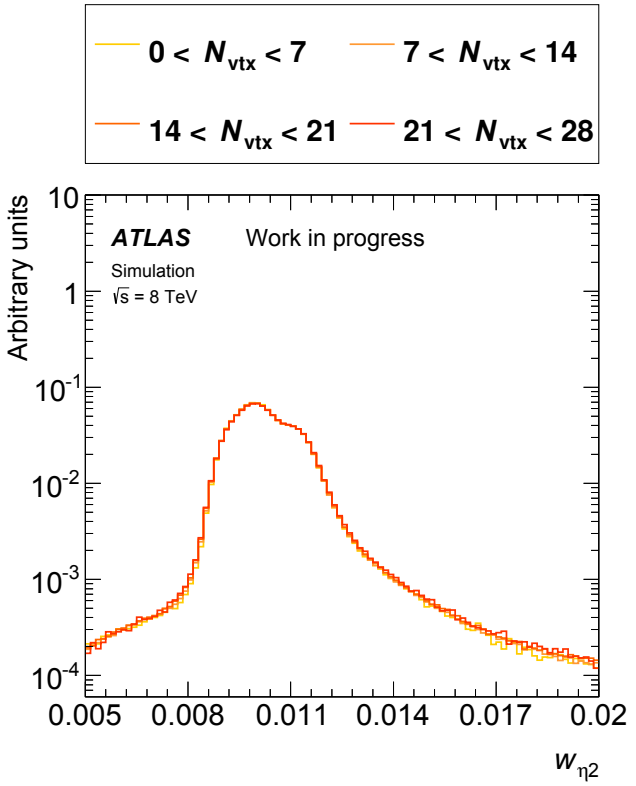

(d) Pileup dependence

FIGURE 4.5: $w_{\eta 2}$ distributions of reconstructed electron candidates. The distributions are shown in (a) for isolated electrons, as well as the major background components. Figures (b) - (d) illustrate the dependence of the distributions of isolated electrons on $E_{\mathrm{T}},|\eta|$, and pileup. 

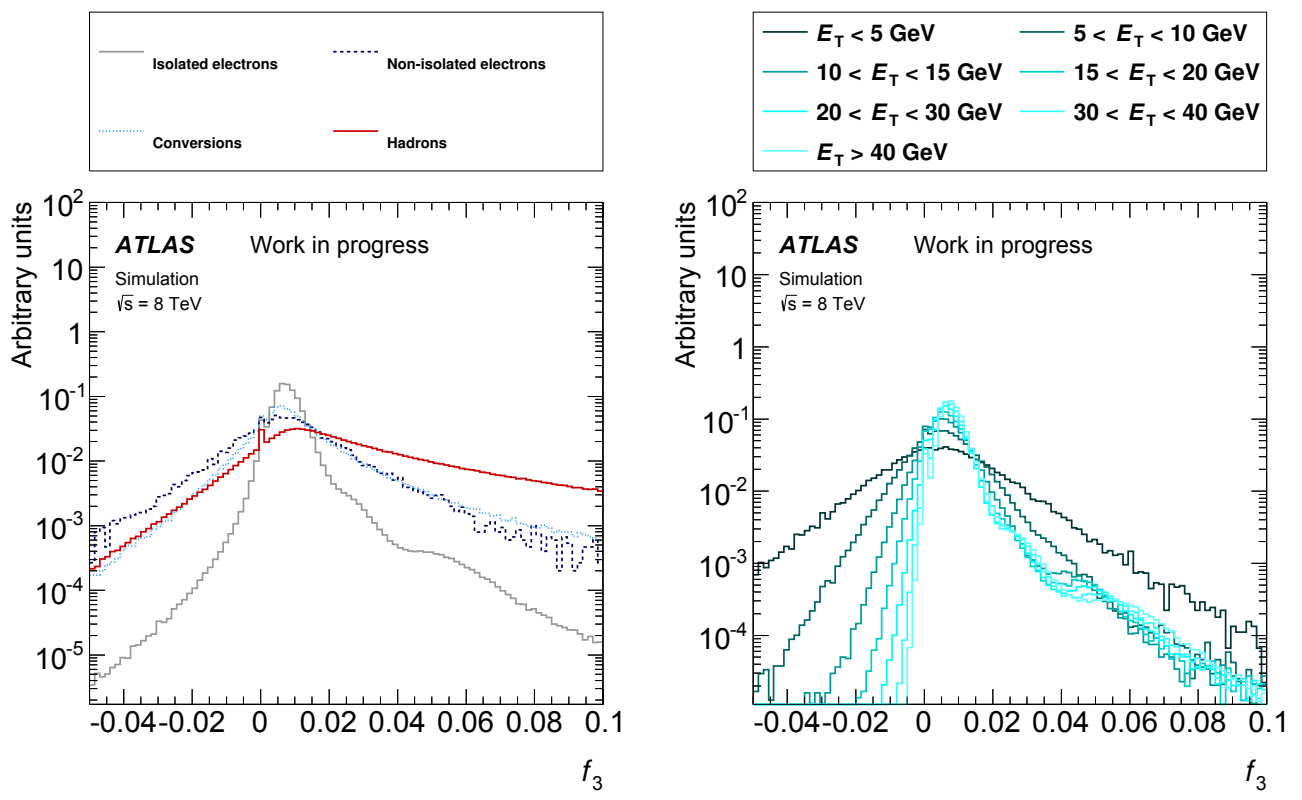

(a) Composition dependence

(b) $E_{\mathrm{T}}$ dependence
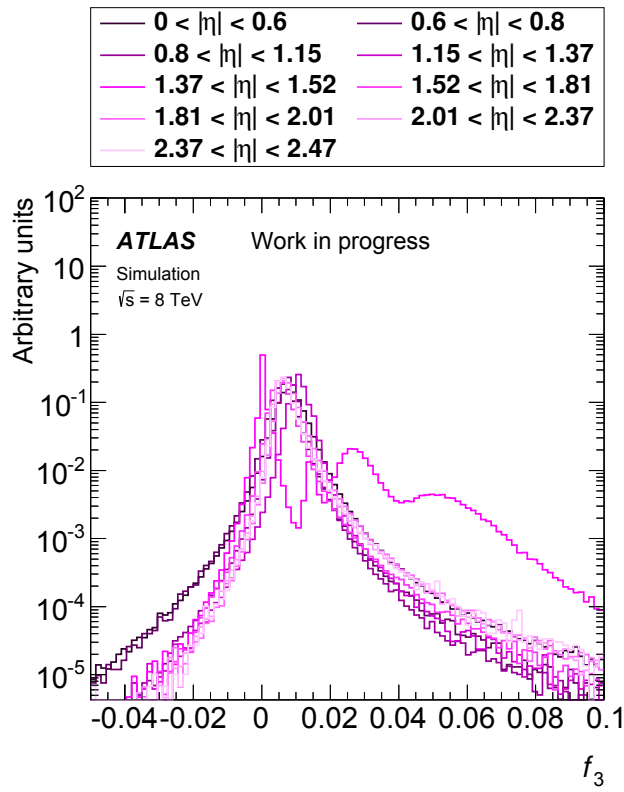

(c) $|\eta|$ dependence
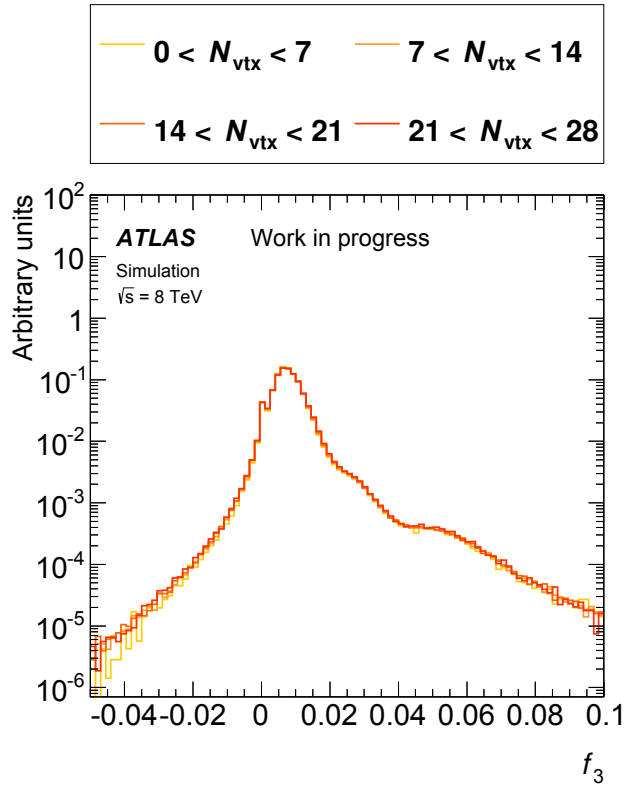

(d) Pileup dependence

FiguRE 4.6: $f_{3}$ distributions of reconstructed electron candidates. The distributions are shown in (a) for isolated electrons, as well as the major background components. Figures (b) - (d) illustrate the dependence of the distributions of isolated electrons on $E_{\mathrm{T}},|\eta|$, and pileup. 
- $\boldsymbol{R}_{\text {had }}$ Also known as the hadronic leakage, this quantity describes the ratio of the $E_{\mathrm{T}}$ deposited in the first layer of the hadronic calorimeter to the EM cluster $E_{\mathrm{T}}$ :

$$
R_{\mathrm{had}}=\frac{E_{\mathrm{T}}^{\mathrm{had}}}{E_{\mathrm{T}}} .
$$

This quantity should be small $(\sim 0)$ for true electrons, which are contained within the EM calorimeter, but hadrons misreconstructed as electrons tend to display significant energy leakage in the hadronic calorimeter. As shown in Figure $4.7, R_{\text {had }}$ depends strongly on pileup conditions, since the presence of jets due to pileup leads to additional energy being deposited in the hadronic calorimeter. $R_{\text {had }}$ is also significantly affected by the cluster $E_{\mathrm{T}}$ and $|\eta|$.

\subsubsection{Track quality variables}

- $\boldsymbol{N}_{\text {pix }}$ Photons tend to leave the pixel detector before converting, so they generally do not leave hits in the pixel detector, as seen in Figure 4.8. To distinguish true electrons from photon conversions, there should be at least one hit in the pixel detector, regardless of $E_{\mathrm{T}},|\eta|$ or pileup conditions.

- $\boldsymbol{N}_{\mathbf{S i}}$ To provide additional discriminating power against conversions, at least seven hits should occur in the silicon detectors. The distribution of $N_{\mathrm{Si}}$ is shown in Figure 4.8.

- $\boldsymbol{R}_{\text {TRT }}$ This is defined as the fraction of high-threshold hits in the TRT. Electrons typically produce more high-threshold hits due to the emission of transition radiation. Hadrons, which emit much less transition radiation, typically have a much lower value of $R_{\mathrm{TRT}}$, as shown in Figure 4.9. This 

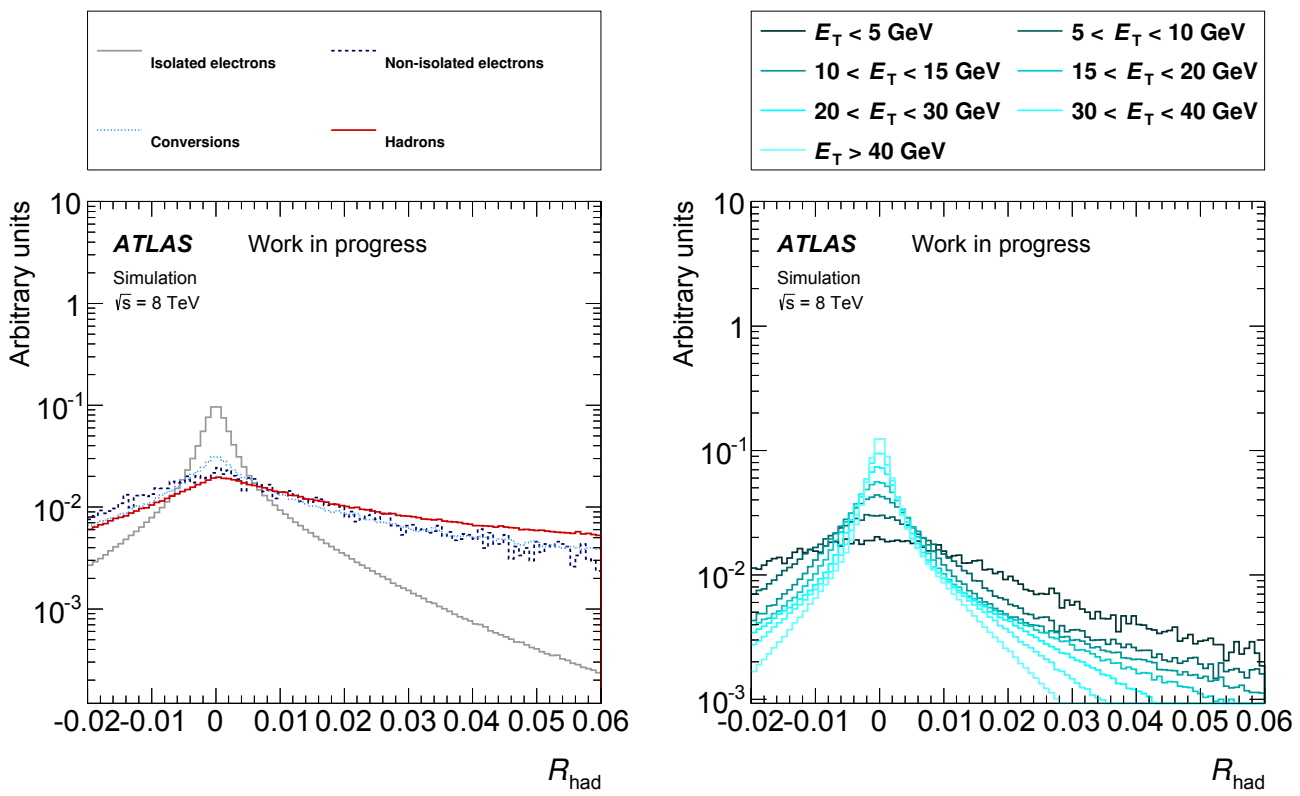

(a) Composition dependence

(b) $E_{\mathrm{T}}$ dependence
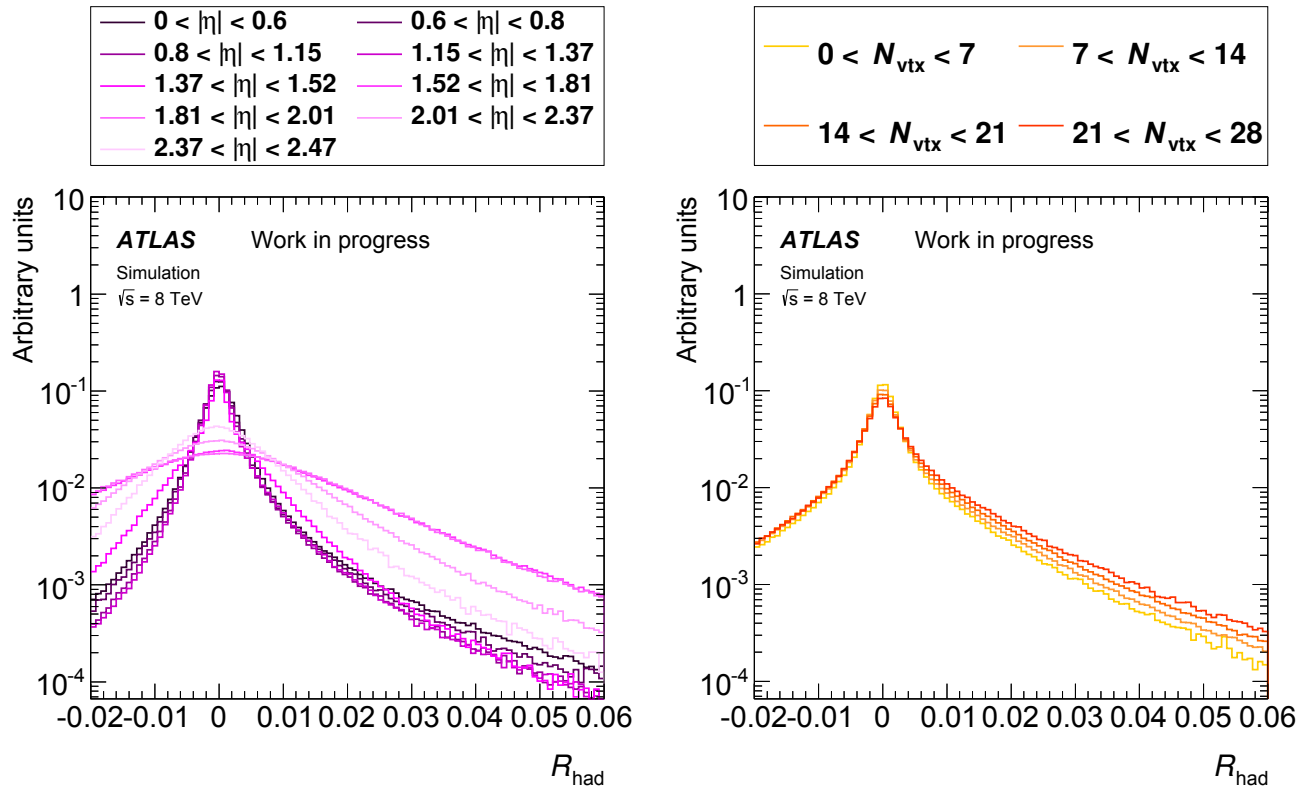

(c) $|\eta|$ dependence

(d) Pileup dependence

Figure 4.7: $R_{\text {had }}$ distributions of reconstructed electron candidates. The distributions are shown in (a) for isolated electrons, as well as the major background components. Figures (b) - (d) illustrate the dependence of the distributions of isolated electrons on $E_{\mathrm{T}},|\eta|$, and pileup. 


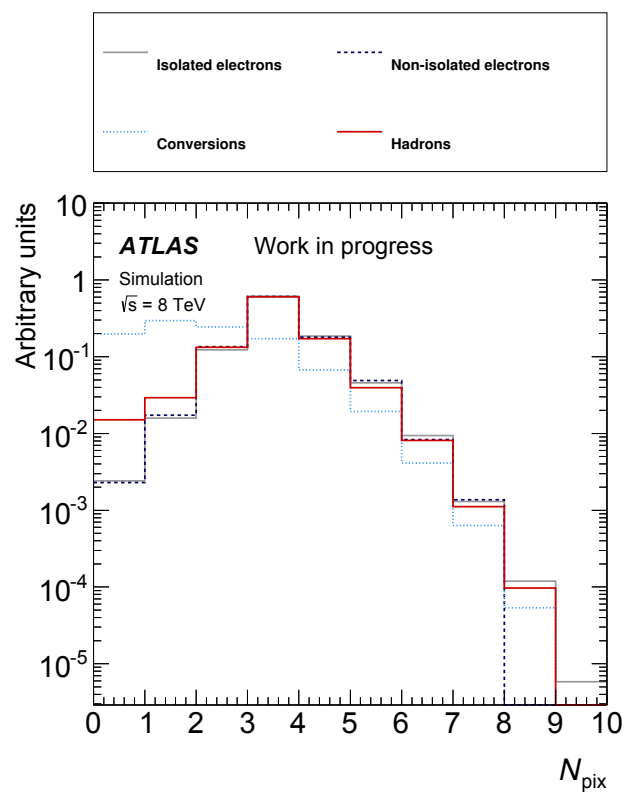

(a) $N_{\text {pix }}$

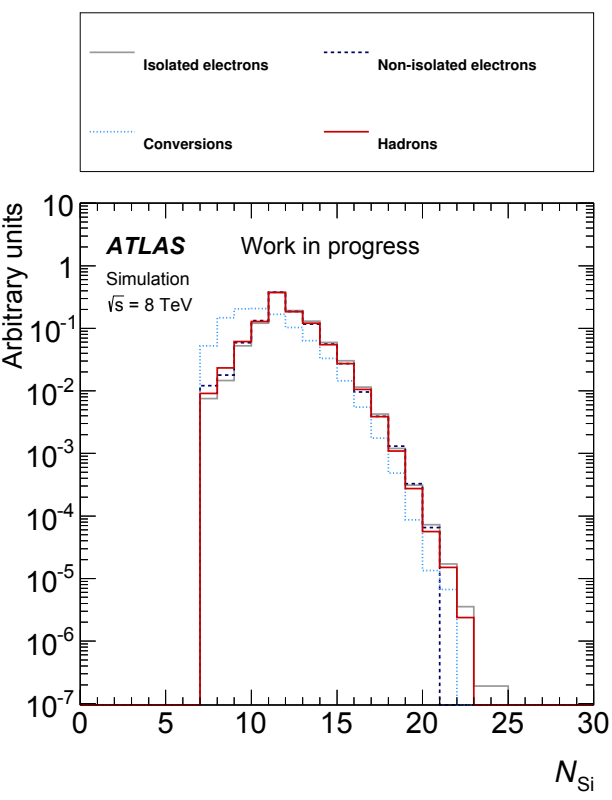

(b) $N_{\mathrm{Si}}$

Figure 4.8: $N_{\text {pix }}$ and $N_{\mathrm{Si}}$ distributions of reconstructed electron candidates. The distributions are shown for isolated electrons, as well as the major background components.

variable is not used above $|\eta|=2$ since the TRT coverage does not extend to this region.

- $\boldsymbol{\Delta} \boldsymbol{p} / \boldsymbol{p}$ This variable exploits new information made available by the GSF electron reconstruction algorithm (introduced in Section 3.3.1) and was not used prior to 2012. It is defined as the relative difference between the track's momentum at the perigee (i.e. the point of closest approach to the primary vertex) and upon exiting the inner detector:

$$
\Delta p / p=\frac{p_{\text {init }}-p_{\text {final }}}{p_{\text {final }}} .
$$

Electrons, which lose momentum due to bremsstrahlung emission as they traverse the inner detector, often have significant values of $\Delta p / p$, while hadrons, 

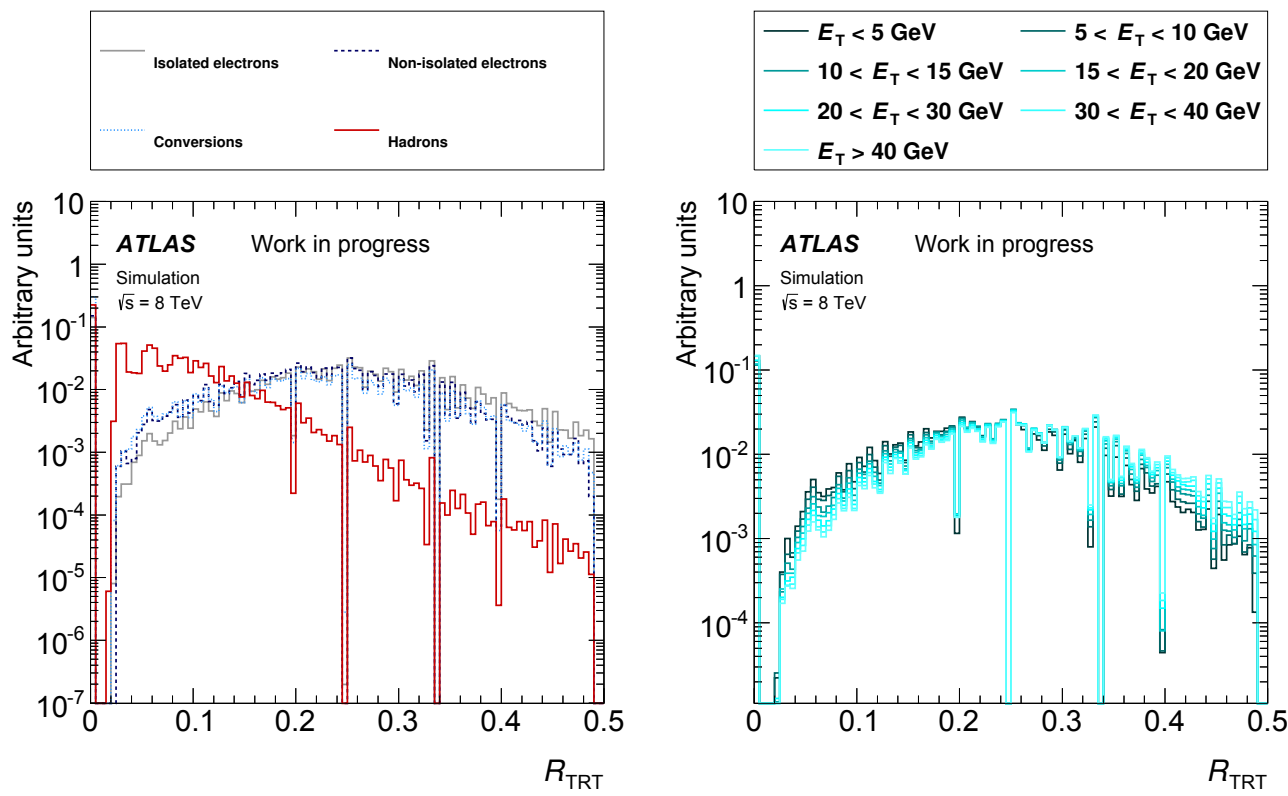

(a) Composition dependence

(b) $E_{\mathrm{T}}$ dependence
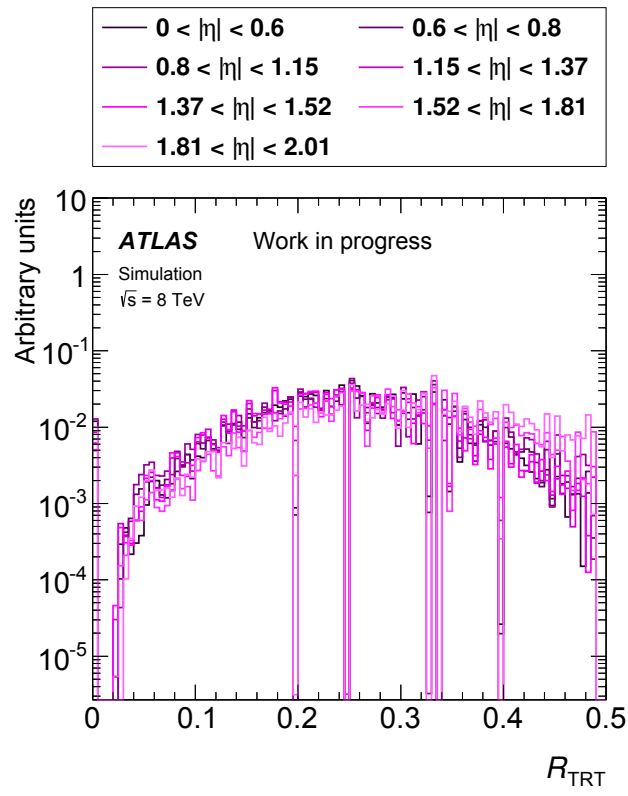

(c) $|\eta|$ dependence
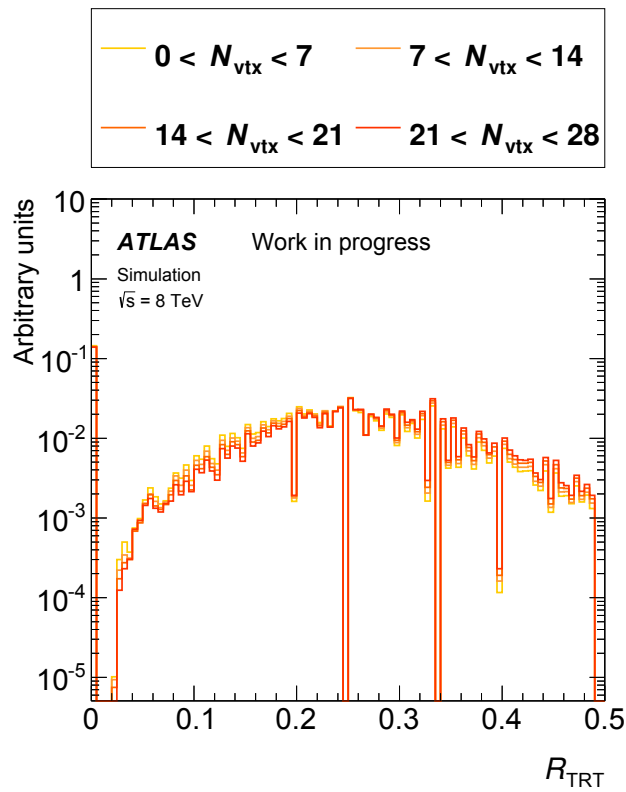

(d) Pileup dependence

FIgURE 4.9: $R_{\mathrm{TRT}}$ distributions of reconstructed electron candidates. The distributions are shown in (a) for isolated electrons, as well as the major background components. Figures (b) - (d) illustrate the dependence of the distributions of isolated electrons on $E_{\mathrm{T}},|\eta|$, and pileup. 
which emit little bremsstrahlung, tend to have $\Delta p / p \sim 0$, as seen in Figure 4.10. It is not strongly affected by $E_{\mathrm{T}}$ or by pileup conditions, but it does depend on $|\eta|$; i.e. on the amount of material traversed by the particle.

\subsubsection{Track-cluster matching variables}

- $\Delta \boldsymbol{\eta}_{\mathbf{1}}$ This quantity is defined as the difference in $\eta$ between the EM calorimeter cluster centroid and the inner detector track, extrapolated to the first layer of the calorimeter. As can be seen in Figure 4.11, true, isolated electrons have very well-matched tracks, yielding a narrow distribution peaked at zero, whereas non-isolated background objects and conversions tend to have $\Delta \eta_{1}$ distributions with much broader tails. $\Delta \eta_{1}$ is significantly affected by $E_{\mathrm{T}}$ and $|\eta|$.

- $\Delta \phi_{\mathbf{R}}$ This variable measures the quality of the track-cluster matching in $\phi$ in the second layer of the EM calorimeter. The value of the track momentum is replaced by the cluster energy when extrapolating the track to the second layer of the EM calorimeter, which results in a relatively symmetric distribution. Since this variable is measured in the bending plane of the magnetic field, it is highly sensitive to bremsstrahlung emission. Isolated electrons tend have a very narrow $\Delta \phi_{\mathrm{R}}$ distribution, while the distributions for background objects are much broader, especially for hadrons, as shown in Figure 4.12. $\Delta \phi_{\mathrm{R}}$ is not affected by pileup, but is dependent on $E_{\mathrm{T}}$ and $|\eta|$. 

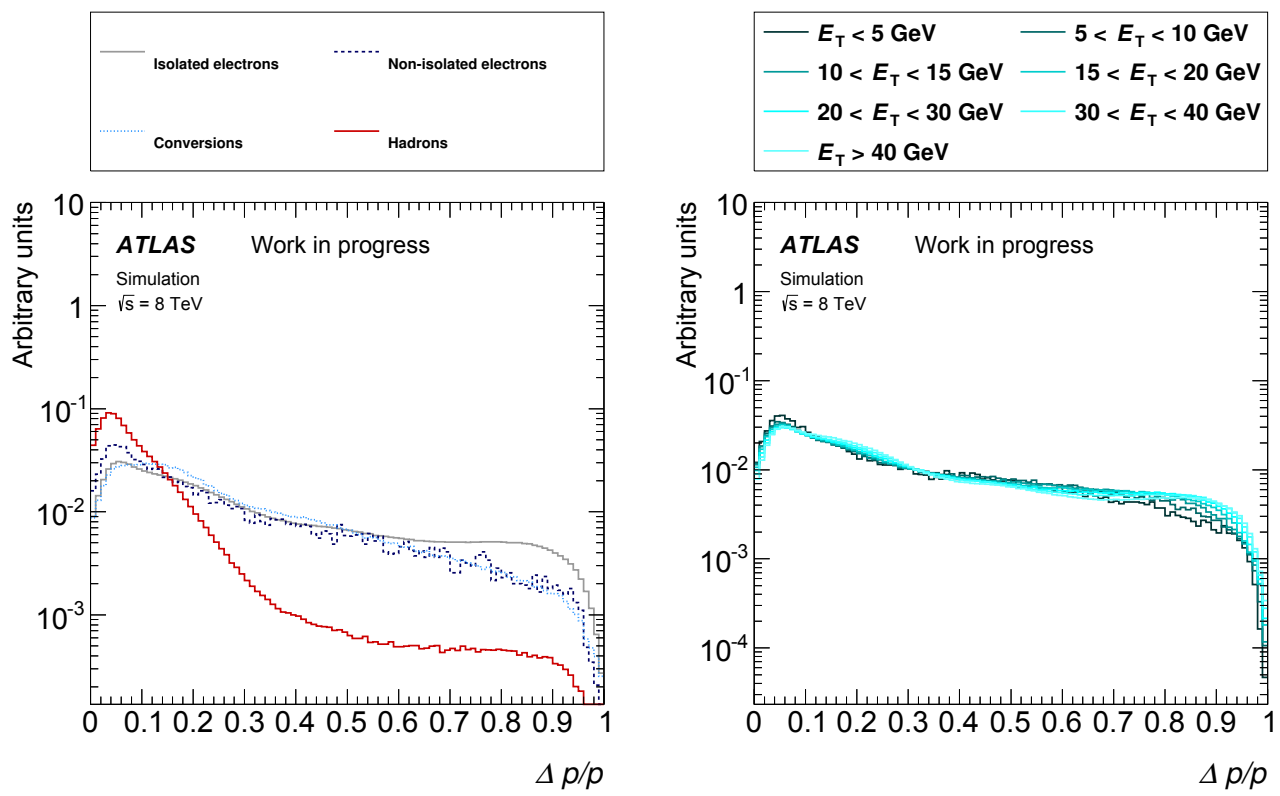

(a) Composition dependence

(b) $E_{\mathrm{T}}$ dependence
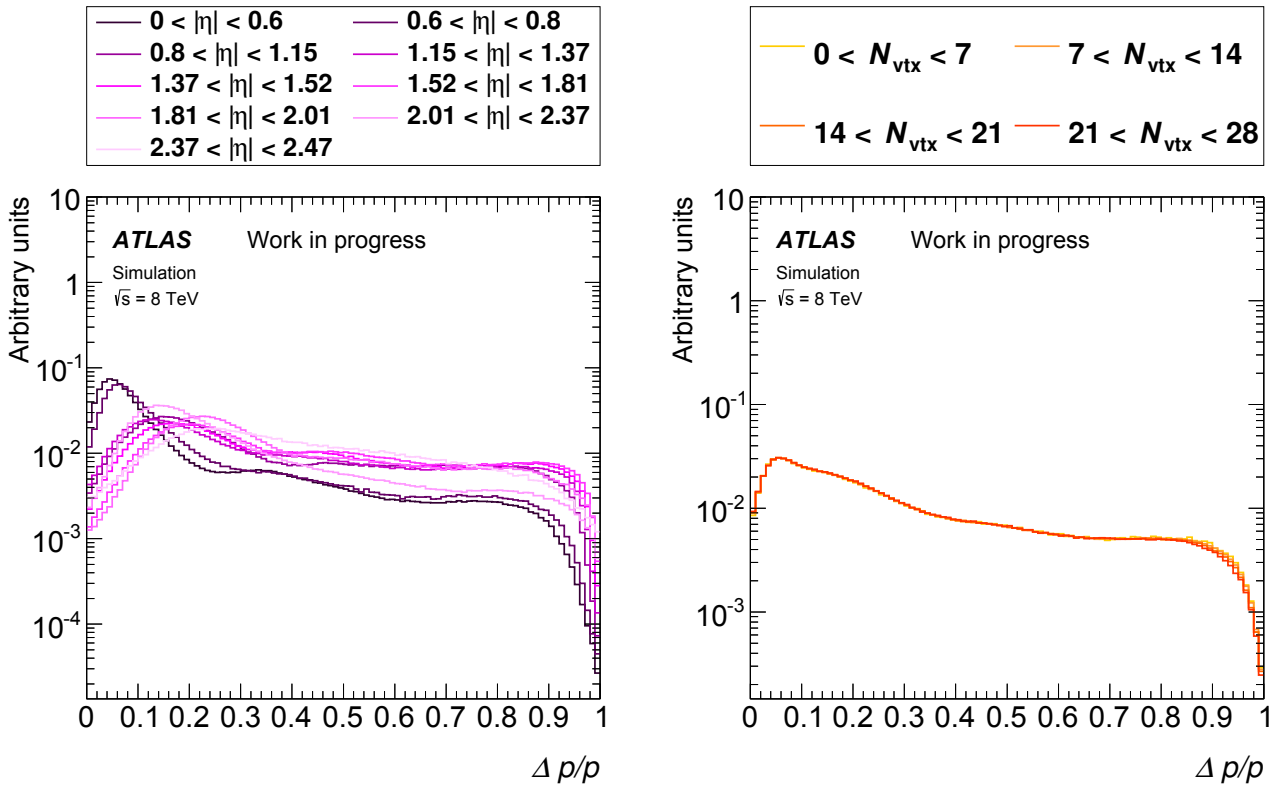

(c) $|\eta|$ dependence

(d) Pileup dependence

Figure 4.10: $\Delta p / p$ distributions of reconstructed electron candidates. The distributions are shown in (a) for isolated electrons, as well as the major background components. Figures (b) - (d) illustrate the dependence of the distributions of isolated electrons on $E_{\mathrm{T}},|\eta|$, and pileup. 

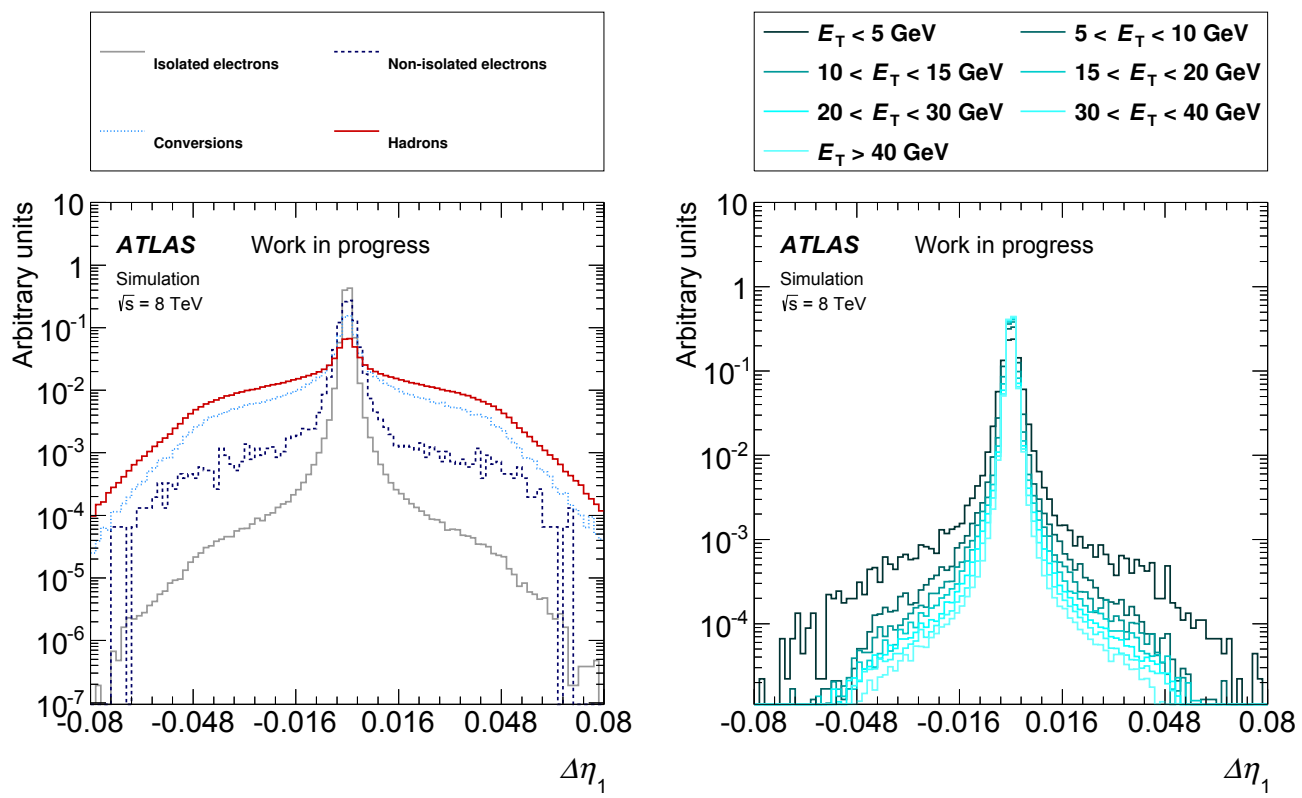

(a) Composition dependence

(b) $E_{\mathrm{T}}$ dependence
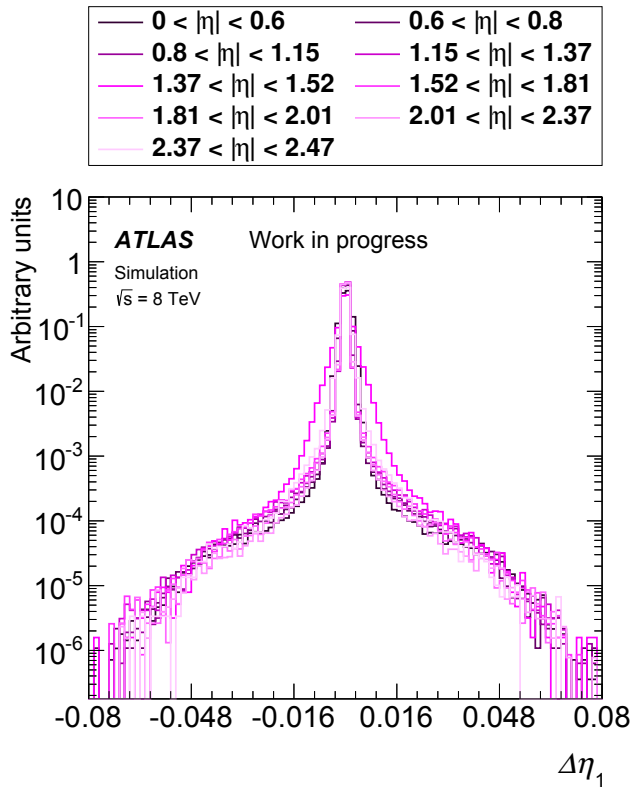

(c) $|\eta|$ dependence
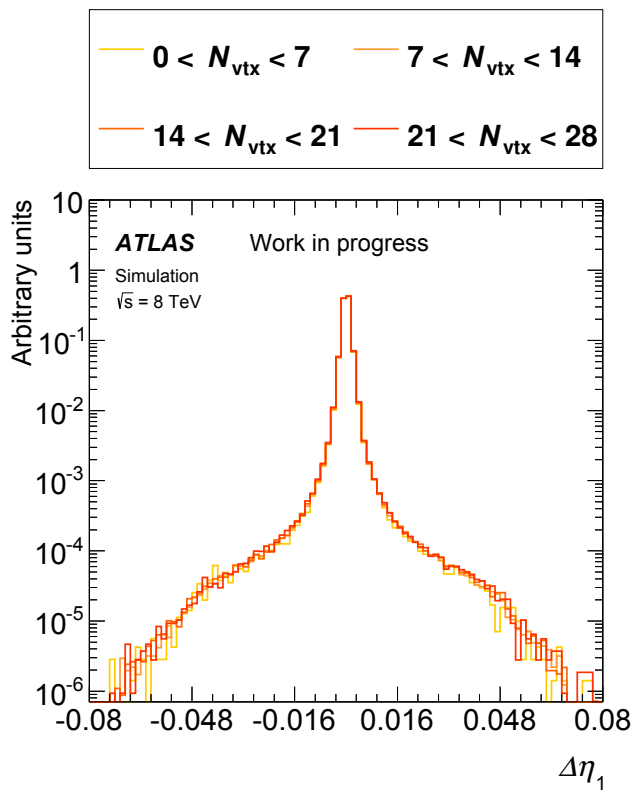

(d) Pileup dependence

Figure 4.11: $\Delta \eta_{1}$ distributions of reconstructed electron candidates. The distributions are shown in (a) for isolated electrons, as well as the major background components. Figures (b) - (d) illustrate the dependence of the distributions of isolated electrons on $E_{\mathrm{T}},|\eta|$, and pileup. 

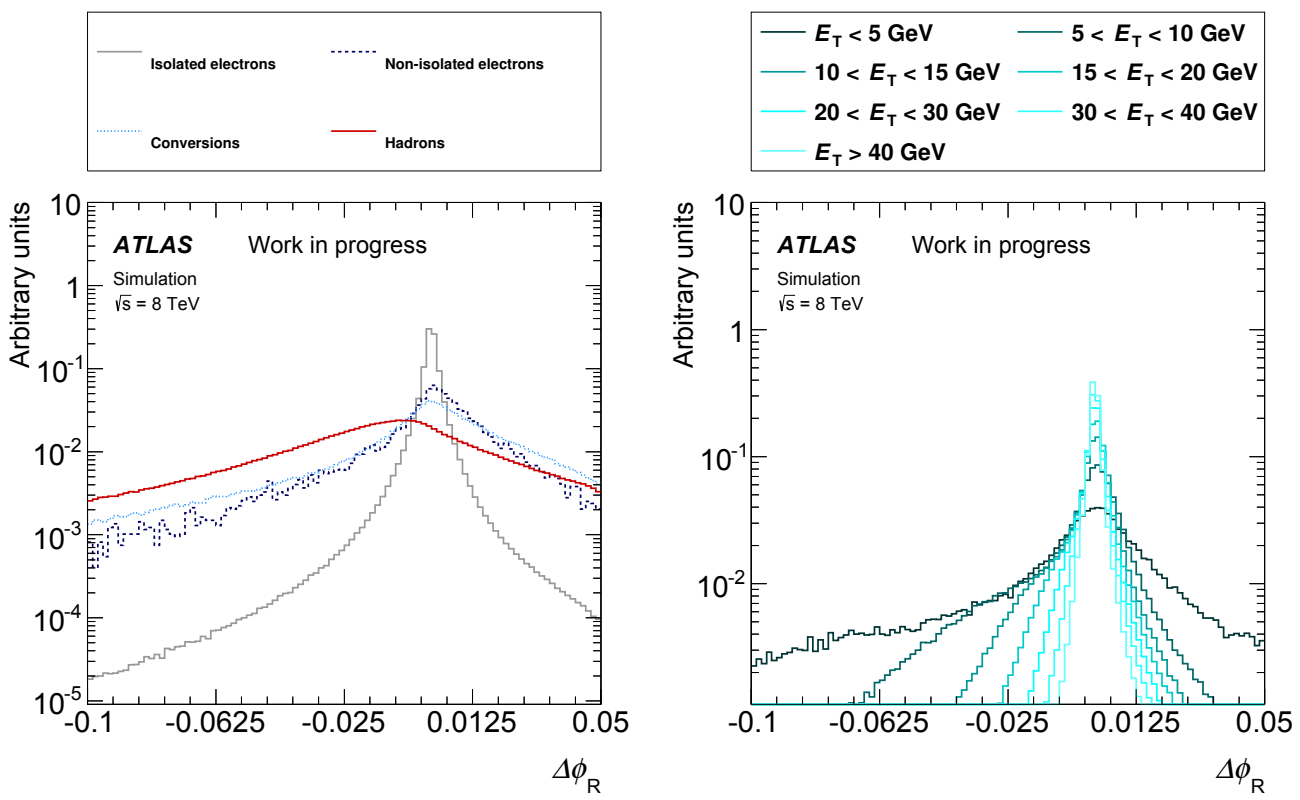

(a) Composition dependence

(b) $E_{\mathrm{T}}$ dependence
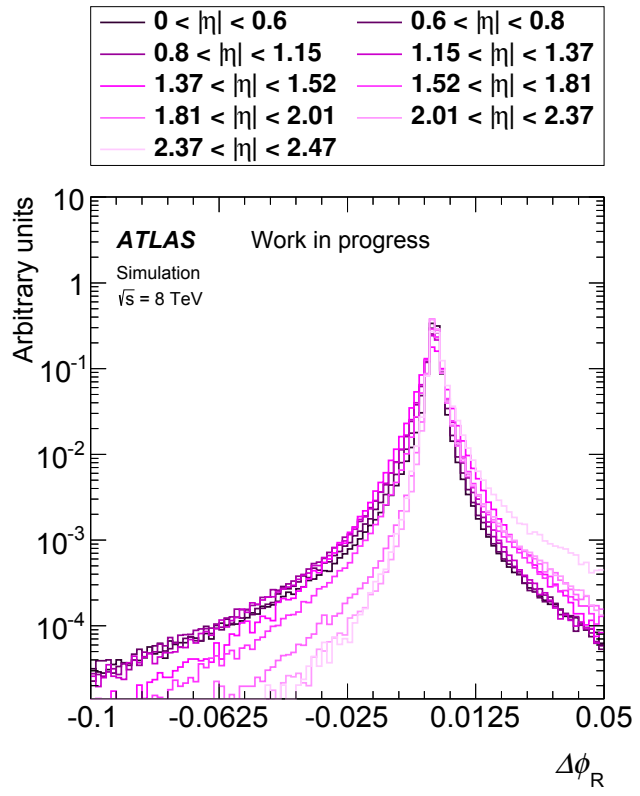

(c) $|\eta|$ dependence
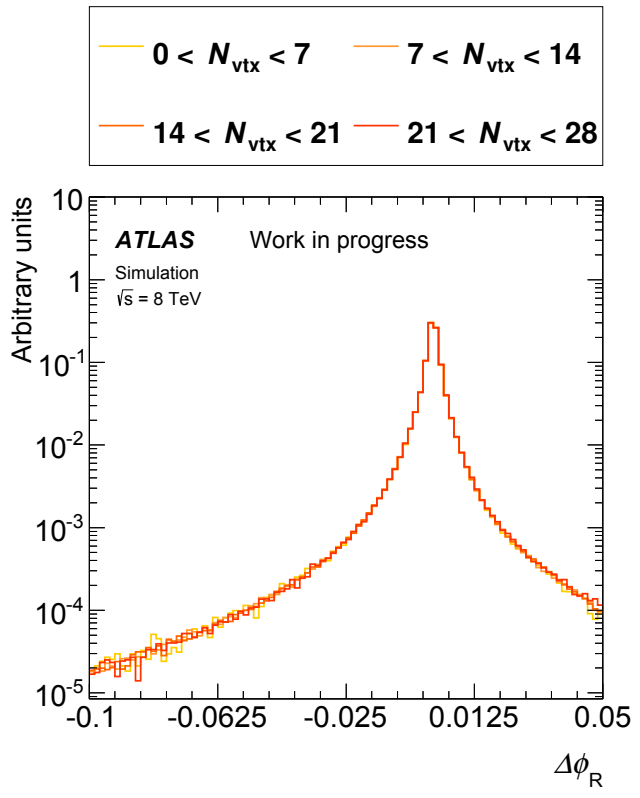

(d) Pileup dependence

FiguRE 4.12: $\Delta \phi_{\mathrm{R}}$ distributions of reconstructed electron candidates. The distributions are shown in (a) for isolated electrons, as well as the major background components. Figures (b) - (d) illustrate the dependence of the distributions of isolated electrons on $E_{\mathrm{T}},|\eta|$, and pileup. 


\subsection{The isEM ++ menu}

The standard menu used for electron identification is known as the IsEM++ menu [47]. It features multiple operating points with progressively higher degrees of background rejection and correspondingly decreasing degrees of signal efficiency: Loose,++ Medium ++ , and Tight ++ .

For the $7 \mathrm{TeV} H \rightarrow Z Z^{(*)} \rightarrow 4 \ell$ analysis, the Loose++ operating point was used. This operating point consisted of cuts, binned in $|\eta|$ and $E_{\mathrm{T}}$ on the shower shape variables: $R_{\text {had }}, R_{\eta}, w_{\eta 2}, w_{\mathrm{s} \text {, tot }}$, and $E_{\text {ratio }}$, as well as the basic track quality requirements: $N_{\text {pix }} \geq 1$ and $N_{\mathrm{Si}} \geq 7$. A flat (unbinned) cut was also applied on $\Delta \eta_{1}: \Delta \eta_{1}<0.015$. This menu provided approximately $93 \%$ identification efficiency, and a hadron rejection factor of about 25 .

However, the Loose ++ operating point was not optimized for high-pileup conditions nor for low- $E_{\mathrm{T}}$ electrons, which made it less powerful for the $8 \mathrm{TeV}$ analysis. For this round of the analysis, which featured reoptimized cuts, the efficiency was slightly higher, at about 95\%, but the hadron rejection factor was markedly lower $(\sim 14)$. The efficiency and rejection also displayed some dependence on the number of primary vertices, which was troubling given that the expected pileup conditions for the 2012 run were not known with absolute certainty at the beginning of the year.

For these reasons, it was decided to use a custom-designed electron identification menu for the $8 \mathrm{TeV} H \rightarrow Z Z^{(*)} \rightarrow 4 \ell$ analysis; this menu was the author's major contribution to the discovery of the Higgs boson, and will be discussed in detail in the next section. 


\subsection{Offline electron identification in the $H \rightarrow$ $Z Z^{(*)} \rightarrow 4 \ell$ search: the MultiLepton menu}

\subsubsection{Motivation for the MultiLepton menu}

In order to optimize the $H \rightarrow Z Z^{(*)} \rightarrow 4 \ell$ analysis in preparation for the $8 \mathrm{TeV}$ run, it was determined that it would be necessary to replace the LoOsE ++ menu with one specifically designed to maximize the discovery potential. Since this particular process is quite rare, with numerous sources of background electrons and hadrons, the analysis required both high signal efficiency and high background rejection, which no single ISEM ++ menu could provide.

With these considerations in mind, a customized menu, which became known as the MultiLepton menu [3], was designed and implemented. The strategy employed in designing this menu took the following objectives into account:

- Increased efficiency at low $\boldsymbol{E}_{\mathrm{T}}$ At low electron $E_{\mathrm{T}}$, the identification efficiency of the Loose ++ menu drops off sharply. It was necessary to reduce this loss to improve the likelihood of a discovery in the case of a low-mass Higgs boson, since the analysis accepts electrons with transverse energies as low as $7 \mathrm{GeV}$.

- Pileup robustness The pileup conditions were expected to be much more extreme in 2012, compared to 2011 (recall Figure 3.3). However, the exact pileup conditions in the detector were not known with complete certainty in 
advance of the data-taking, and so it was crucial that the electron identification efficiency and background rejection be constant as a function of the number of primary vertices (a measure of pileup) in a given event.

- Improved hadron rejection Since the background to the $H \rightarrow Z Z^{(*)} \rightarrow 4 \ell$ decay is mainly due to hadrons, it was important that the menu could be tuned to maximize hadron rejection.

- Exploit the benefits of the GSF electron reconstruction The GSF algorithm provides additional tracking information in the form of improved track-cluster matching variables, such as $\Delta \phi_{\mathrm{R}}$. In addition, the $\Delta p / p$ variable is a very powerful discriminator. It was desired to exploit these new features, which were not available in the default ISEM ++ menu, in order to improve the background rejection and electron efficiency.

\subsubsection{Implementation of the MultiLepton menu}

Starting from the Loose ++ menu, cuts were optimized individually for each variable, with the goal of achieving an overall efficiency of $95 \%$. In order to take into account the dependence of each variable on $E_{\mathrm{T}}$ and $|\eta|$, the following twodimensional binning was used:

$$
\begin{gathered}
E_{\mathrm{T}}=\{5,10,15,20,30,40,50,60,70,80+\}[\mathrm{GeV}] . \\
|\eta|=\{0,0.1,0.6,0.8,1.15,1.37,1.52,1.81,2.01,2.37,2.47\} .
\end{gathered}
$$

The $|\eta|$ bins reflect changes in the material depth in the calorimeters, transitions between regions of detector coverage, and changes in the granularity of the strips. 
In some cases, the $0<|\eta|<0.1$ and $0.1<|\eta|<0.6$ bins were merged for statistical reasons, as were the $E_{\mathrm{T}}$ bins above $30-40 \mathrm{GeV}$.

In order to improve the pileup robustness, the most pileup-dependent variables (i.e. $R_{\text {had }}$ and $R_{\eta}$ ) were identified and the cuts on those variables were relaxed, thus yielding higher efficiencies at high pileup. Background rejection was then improved by tightening the cuts on pileup-robust variables.

Cuts were retuned to improve efficiency and rejection, and the discriminating variable $f_{3}$ was added to the menu in order to maximize the rejection of the dominant source of background. In addition, to compensate for the loss of discriminating power due to the inability to use $E_{\text {ratio }}$ and $w_{\mathrm{s} \text {, tot }}$ in the crack region, a cut on $R_{\mathrm{TRT}}$ was added in this region.

The author of this thesis was responsible for implementing several key objectives of the menu, including reoptimization of the $\Delta \eta_{1}$ cut to maximize its hadron-rejecting power. In addition, the use of $\Delta p / p$ as a tool for electron categorization based on bremsstrahlung emission was investigated, resulting in the addition of the bremsstrahlung-sensitive variable $\Delta \phi_{\mathrm{R}}$ with cuts tuned separately for electrons losing significant amounts of momentum due to bremsstrahlung, and for those emitting minimal bremsstrahlung. The optimization of these variables will be discussed in Section 4.3.2.1 and Section 4.3.2.2.

\subsubsection{Optimization of $\Delta \eta_{1}$}

In the Loose ++ menu, a flat, unbinned cut is applied on the track-cluster matching in $\eta: \Delta \eta_{1}<0.015$. However, as can clearly be seen in Figure 4.11, the distribution of $\Delta \eta_{1}$ is highly symmetric, and the tails are significantly broader for the background, compared to isolated electrons. In addition, $\Delta \eta_{1}$ displays a strong 
dependence on $E_{\mathrm{T}}$ and $|\eta|$. This makes $\Delta \eta_{1}$ an ideal candidate for a symmetric, $E_{\mathrm{T}^{-}}$and $|\eta|$-binned cut to exclude the background tails, thus optimizing both its signal efficiency and its background rejection power.

To optimize $\Delta \eta_{1}$, two cuts, symmetric about $\Delta \eta_{1}=0$, were chosen such that an efficiency of $99 \%$ was achieved in each two-dimensional $E_{\mathrm{T}^{-}}|\eta|$ bin for isolated electrons. Several examples of the reoptimized $\Delta \eta_{1}$ cuts in various $E_{\mathrm{T}}$ and $|\eta|$ bins are shown in Figure 4.13, with the old, one-sided Loose ++ cut for comparison, illustrating the improved efficiency and rejection of the new cuts.

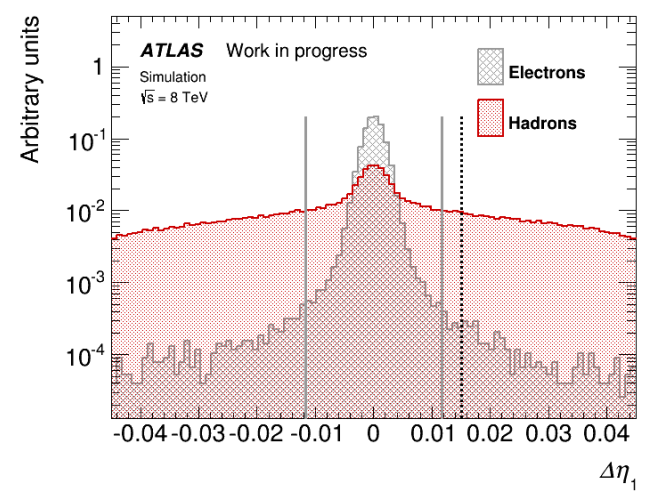

(a) $0<|\eta|<0.6,5 \mathrm{GeV}<E_{\mathrm{T}}<10 \mathrm{GeV}$

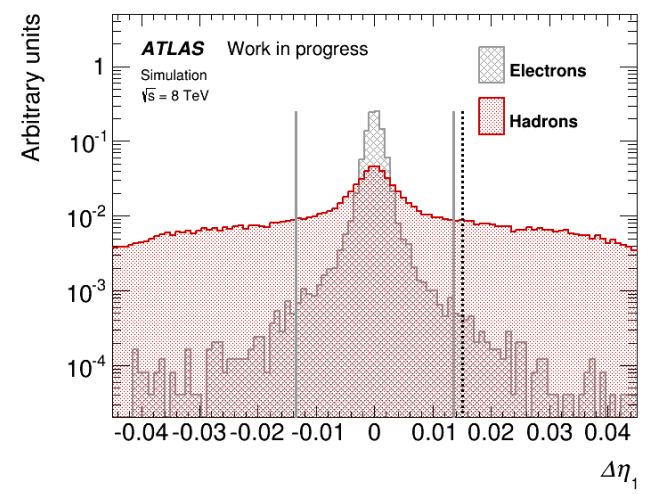

(c) $2.01<|\eta|<2.37,5 \mathrm{GeV}<E_{\mathrm{T}}<10 \mathrm{GeV}$

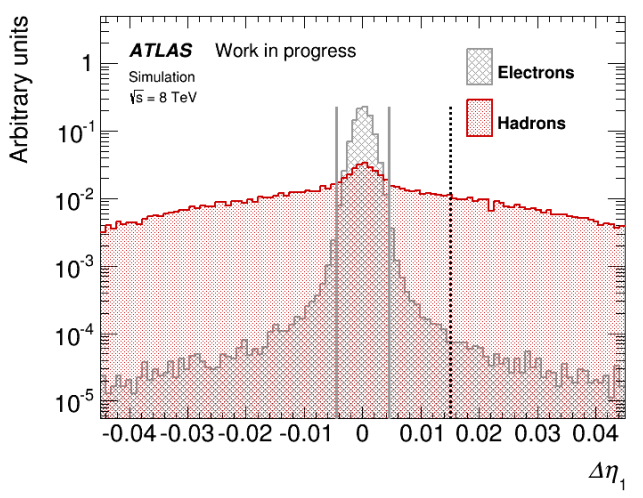

(b) $0<|\eta|<0.6,20 \mathrm{GeV}<E_{\mathrm{T}}<30 \mathrm{GeV}$

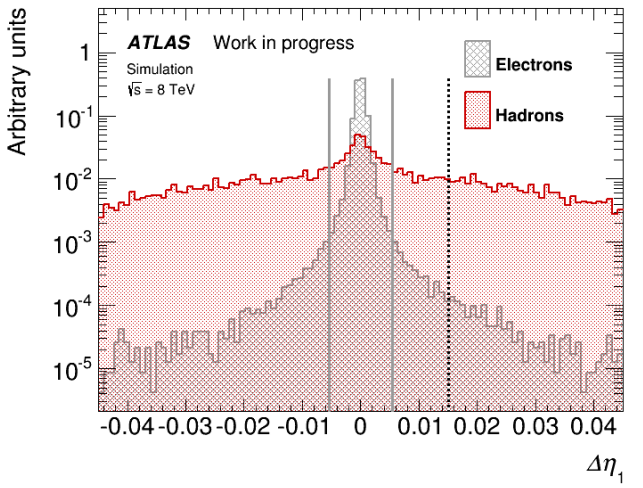

(d) $2.01<|\eta|<2.37,20 \mathrm{GeV}<E_{\mathrm{T}}<30 \mathrm{GeV}$

FiguRE 4.13: Distribution of $\Delta \eta_{1}$ for simulated electrons and hadrons for a selection of high and low values of $E_{\mathrm{T}}$ and $|\eta|$. The solid lines represent the reoptimized, symmetric cuts used in the MultiLePTON menu, while the dashed line represents the flat cut used in the Loose ++ menu. 


\subsubsection{The use of $\Delta p / p$ for electron categorization and the optimiza- tion of $\Delta \phi_{R}$}

As shown in Figure 4.10, $\Delta p / p$ is a powerful discriminating variable for separating electrons from hadrons. In addition, it may also be used to distinguish electrons which have emitted hard (high-energy) bremsstrahlung from those emitting soft (low-energy) or no bremsstrahlung. One of the objectives of the reoptimization of the electron identification menu was to use $\Delta p / p$ to categorize electrons based on their energy losses due to bremsstrahlung. Then, cuts on variables in the bending plane of the magnetic field, such as $\Delta \phi_{\mathrm{R}}$ could be tuned separately for each category, improving the overall efficiency.

The categorization was studied using a simulated sample of electrons and hadrons from $Z+$ jets decays, binned in $E_{\mathrm{T}}$ and $|\eta|$. For each bin, the $\Delta p / p$ distribution of the hadron sample was integrated from $\Delta p / p=0$ up to the value of $\Delta p / p$ at which $95 \%$ of the distribution was contained. Since hadrons emit little bremsstrahlung, the sample of electron objects with $\Delta p / p$ values below this threshold consisted of hadrons and electrons emitting soft or no bremsstrahlung, while the objects above the threshold formed a clean sample of electrons emitting hard bremsstrahlung. An example of this categorization is illustrated in Figure 4.14. $95 \%$ of the hadron $\Delta p / p$ distribution is contained by the dashed line, while the electron distribution features a broad tail above this limit, corresponding to electrons emitting hard bremsstrahlung. The threshold values range from $\Delta p / p \sim 0.1$ at low $|\eta|$ values, to as high as $\Delta p / p \sim 0.7-0.8$ in the high-material region at high values of $|\eta|$.

The $\Delta p / p$ thresholds extracted using this method were then used to separate the electron sample into high- and low-bremsstrahlung categories for the 


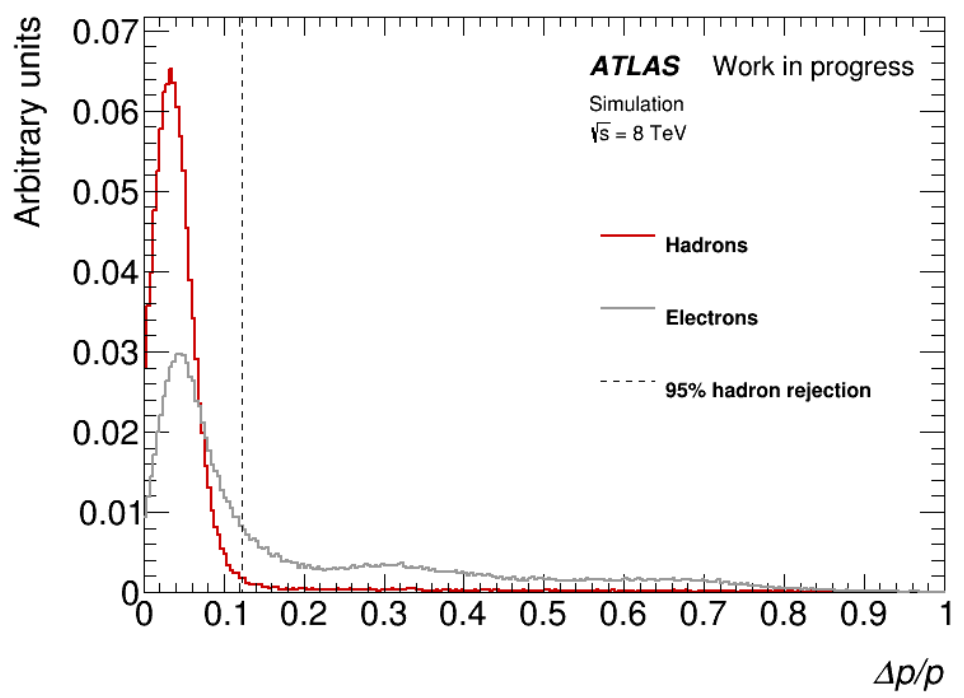

Figure 4.14: The use of $\Delta p / p$ to determine thresholds for bremsstrahlung categorization. $\Delta p / p$ distributions are shown for simulated electrons and hadrons with $10 \mathrm{GeV}<E_{\mathrm{T}}<15 \mathrm{GeV}$ and $0<|\eta|<0.6$. The dashed line represents the threshold below which $95 \%$ of hadrons are contained; this threshold can then be used to separate electrons into hard- and soft-bremsstrahlung categories.

purposes of tuning cuts on $\Delta \phi_{\mathrm{R}}$. Since $\Delta \phi_{\mathrm{R}}$ is in the bending plane, it is sensitive to bremsstrahlung emission; optimizing separate sets of cuts for the two electron categories maximized the overall efficiency and background rejection for this variable. For each $E_{\mathrm{T}^{-}}|\eta|$ bin, asymmetric upper and lower cuts were derived for each category, with the objective of achieving $98.5 \%$ efficiency and maximum background rejection for each cut. Several examples of these cuts can be seen in Figure 4.15 .

\subsubsection{Performance of the MultiLepton menu}

Figure 4.16 compares the performance of the MultiLePton menu with that of the LoOsE ++ menu in terms of electron identification efficiency (defined as the fraction of electron objects passing identification, with respect to the total number 


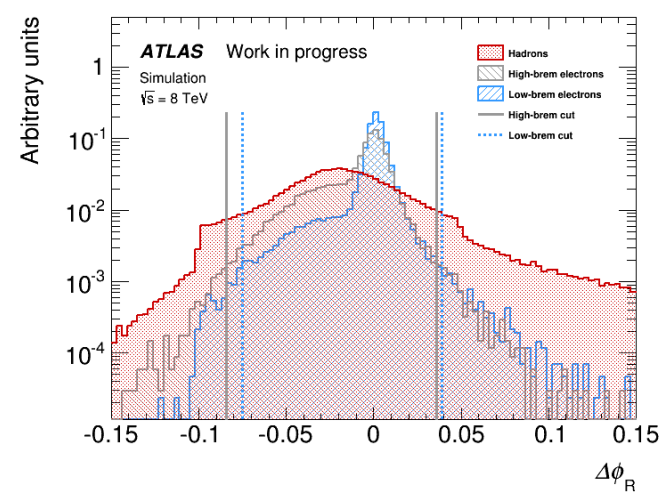

(a) $0<|\eta|<0.6,5 \mathrm{GeV}<E_{\mathrm{T}}<10 \mathrm{GeV}$

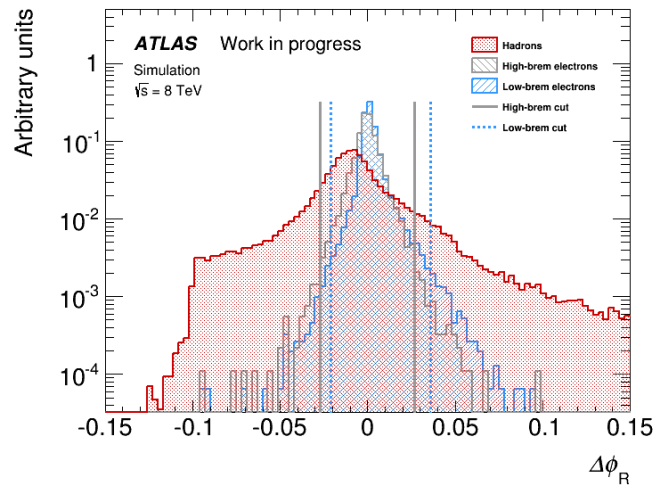

(c) $2.01<|\eta|<2.37,5 \mathrm{GeV}<E_{\mathrm{T}}<10 \mathrm{GeV}$

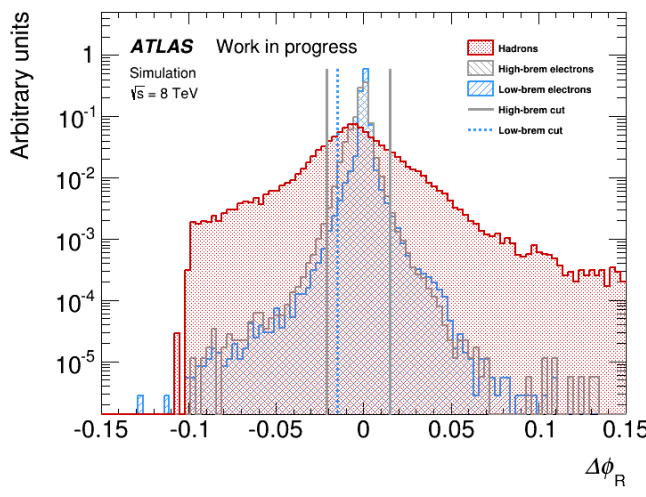

(b) $0<|\eta|<0.6,20 \mathrm{GeV}<E_{\mathrm{T}}<30 \mathrm{GeV}$

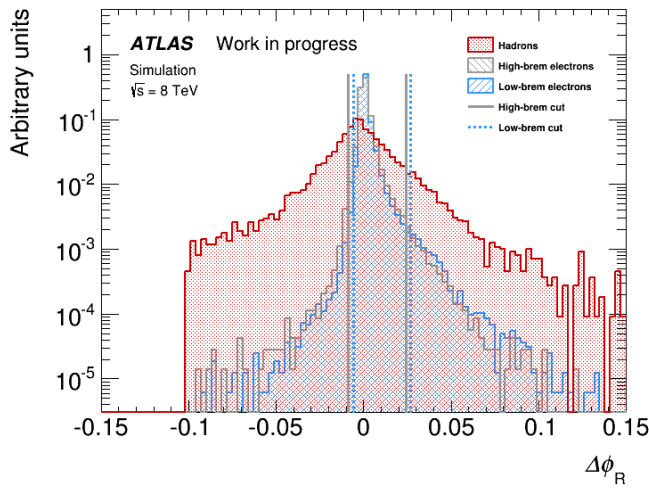

(d) $2.01<|\eta|<2.37,20 \mathrm{GeV}<E_{\mathrm{T}}<30 \mathrm{GeV}$

FiguRE 4.15: Distribution of $\Delta \phi_{\mathrm{R}}$ for electrons and hadrons for a selection of high and low values of $E_{\mathrm{T}}$ and $|\eta|$. The solid (dashed) lines represent the cuts used for electrons emitting hard (soft or no) bremsstrahlung, based on the $\Delta p / p$ threshold below which $95 \%$ of hadrons are contained.

of electron objects in the sample). Both menus have average efficiencies of $\sim 95 \%$, but the MultiLepton menu displays significantly improved efficiency in the crack region $(1.37<|\eta|<1.52)$, as well as at low $E_{\mathrm{T}}$, especially in the range $10 \mathrm{GeV}<$ $E_{\mathrm{T}}<15 \mathrm{GeV}$, thereby achieving the objective of this new menu. The dependence of the efficiency on pileup has also been reduced.

The MultiLepton menu also achieved its goal of achieving major improvements with respect to LoOSE ++ in terms of hadron rejection (defined as 

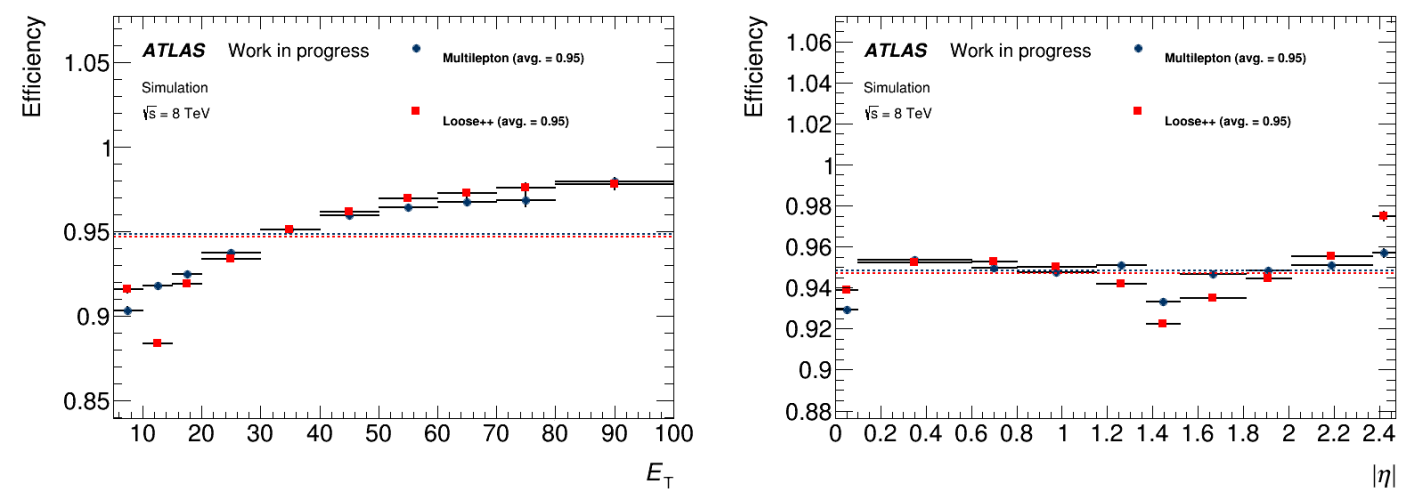

(a) Electron efficiency as a function of $E_{\mathrm{T}}$

(b) Electron efficiency as a function of $|\eta|$

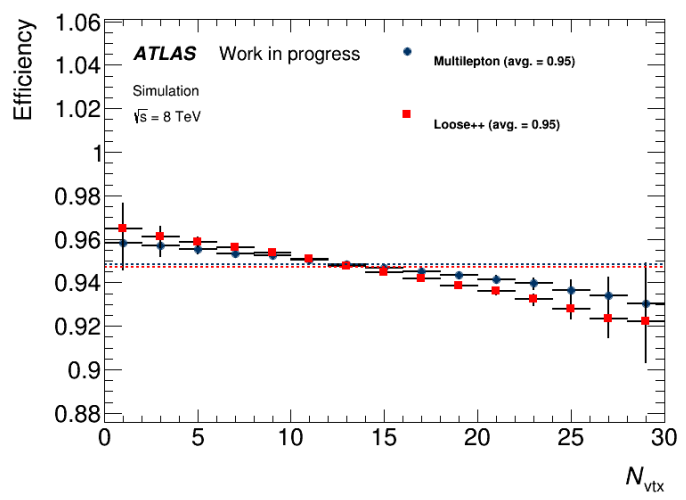

(c) Electron efficiency as a function of $N_{\mathrm{vtx}}$

Figure 4.16: Electron efficiency of the MultiLepton menu, compared to the Loose ++ menu. The efficiencies are shown as a function of $E_{\mathrm{T}},|\eta|$, and $N_{\mathrm{vtx}}$. The dashed lines represent the average efficiency, which corresponds to $95 \%$ for both menus.

the ratio of the total number of hadrons to the number of hadrons passing identification). Figure 4.17 shows the hadron rejection as a function of $E_{\mathrm{T}},|\eta|$ and $N_{\mathrm{vtx}}$ for the MultiLepton and Loose++ menus. With a rejection factor of 25.1, MultiLepton features almost twice the rejection power of LoOSE ++ , which has a rejection factor of 13.7. The rejection factor of MultiLePton is greater than that of Loose ++ in every $E_{\mathrm{T}}$ and $|\eta|$ bin. The rejection is also slightly less dependent on pileup.

Although improved conversion rejection was not one of the main objectives 


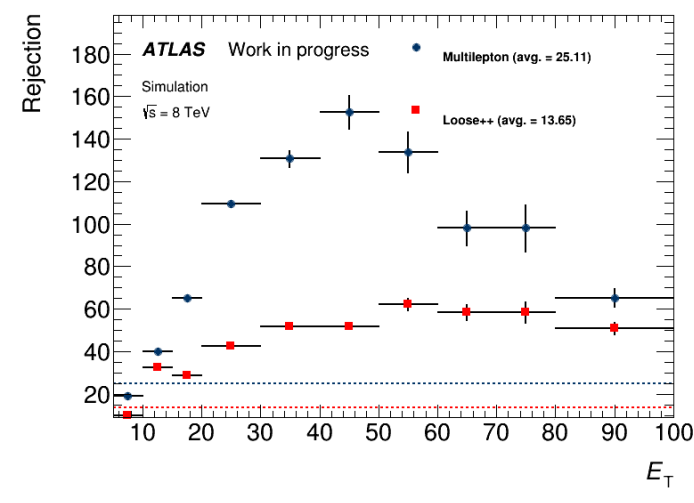

(a) Hadron rejection as a function of $E_{\mathrm{T}}$

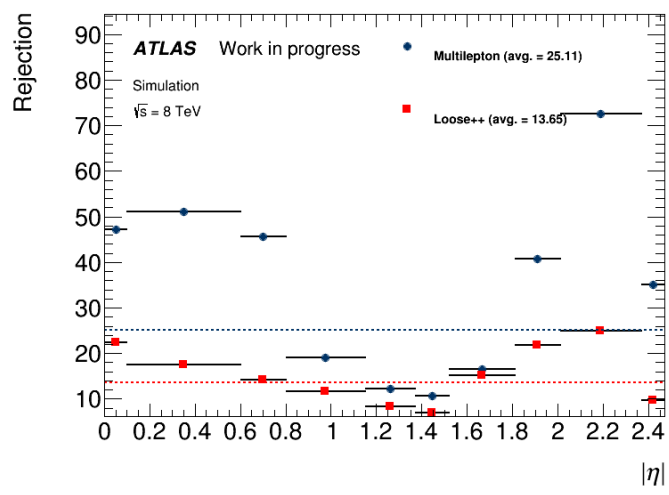

(b) Hadron rejection as a function of $|\eta|$

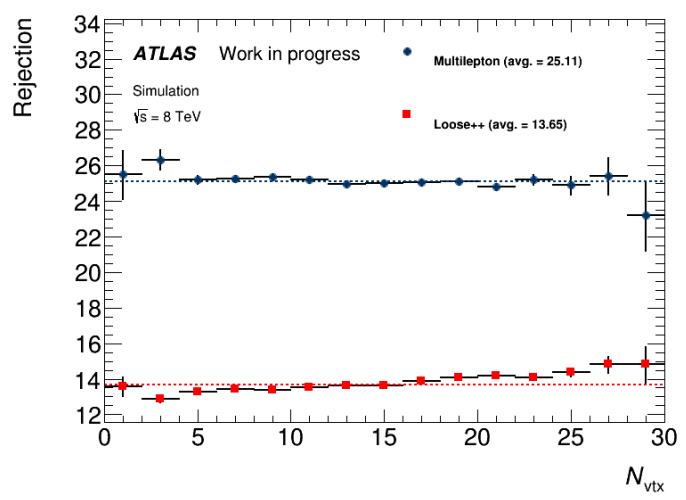

(c) Hadron rejection as a function of $N_{\mathrm{vtx}}$

Figure 4.17: Hadron rejection of the MultiLePton menu, compared to the Loose ++ menu. The dashed lines represent the average rejection, which corresponds to a factor of 25.11 for the MultiLepton menu, and 13.65 for the Loose ++ menu.

of the MultiLepton menu, it also slightly outperforms Loose ++ in this area, having an average rejection factor (defined as the ratio of the total number of conversions to the number of conversions passing identification) of 4.7, compared to 4.4 for Loose ++ . The conversion rejection factors as a function of $E_{\mathrm{T}},|\eta|$, and $N_{\mathrm{vtx}}$ are compared for the two menus in Figure 4.18.

The MultiLepton menu was implemented in 2012, and was used for the analysis of the $8 \mathrm{TeV}$ dataset leading to the discovery of the Higgs boson [2]. As data-taking continued following the discovery and the needs of the analysis evolved, 

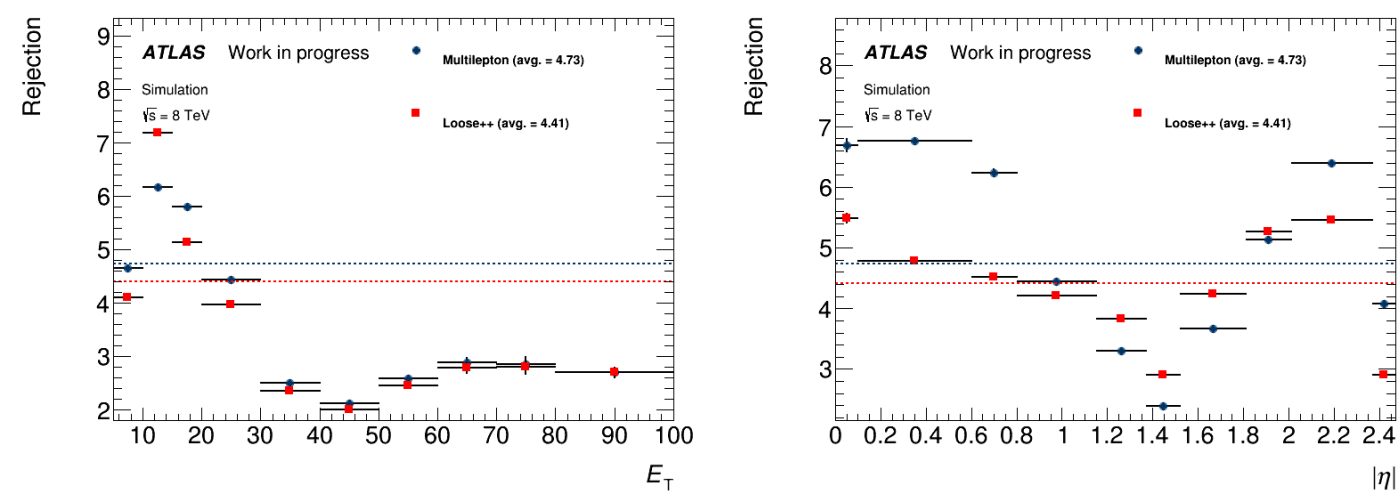

(a) Conversion rejection as a function of $E_{\mathrm{T}}$

(b) Conversion rejection as a function of $|\eta|$

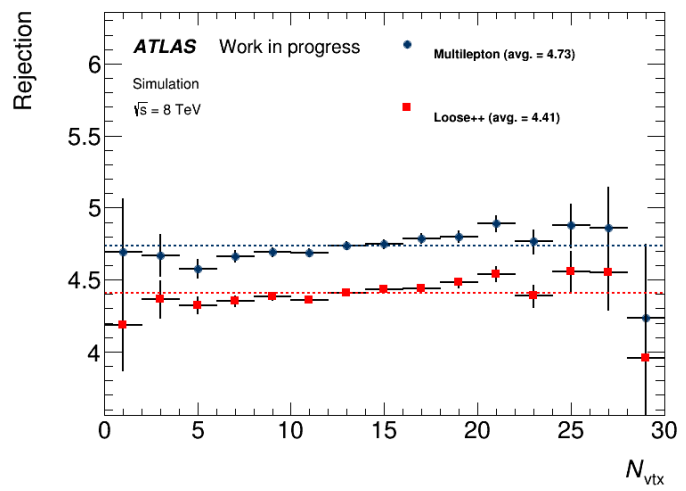

(c) Conversion rejection as a function of $N_{\mathrm{vtx}}$

Figure 4.18: Conversion rejection of the MultiLepton menu, compared to the Loose ++ menu. The dashed lines represent the average rejection, which corresponds to a factor of 4.73 for the MultiLepton menu, and 4.41 for the LoOse ++ menu.

the MultiLepton menu was modified slightly in order to improve background rejection even further, in order to achieve the cleanest possible signal. In particular, improvements were desired in the high-material region $(0.8<|\eta|<1.81)$, where the rejection factor was the lowest (which resulted in a larger degree of background contamination), as seen in Figure 4.17 and Figure 4.18.

To accomplish this, the cuts on $R_{\eta}, \Delta \eta_{1}$, and $\Delta \phi_{\mathrm{R}}$ were tightened slightly for low- $E_{\mathrm{T}}$ bins $\left(E_{\mathrm{T}}<15 \mathrm{GeV}\right)$ in the high-material region, sacrificing a small degree of efficiency for the sake of increased background rejection. In addition, to 
improve conversion rejection, the minimum number of pixel hits was increased from one to two, and a B-layer hit requirement $\left(N_{\mathrm{BL}}>0\right)$ was added to the menu. The B-layer is the inner detector layer located closest to the beamline. True electrons will leave a hit in this layer, while most photons have not yet converted at this point and so they will not have a B-layer hit; this can be seen in Figure 4.19.

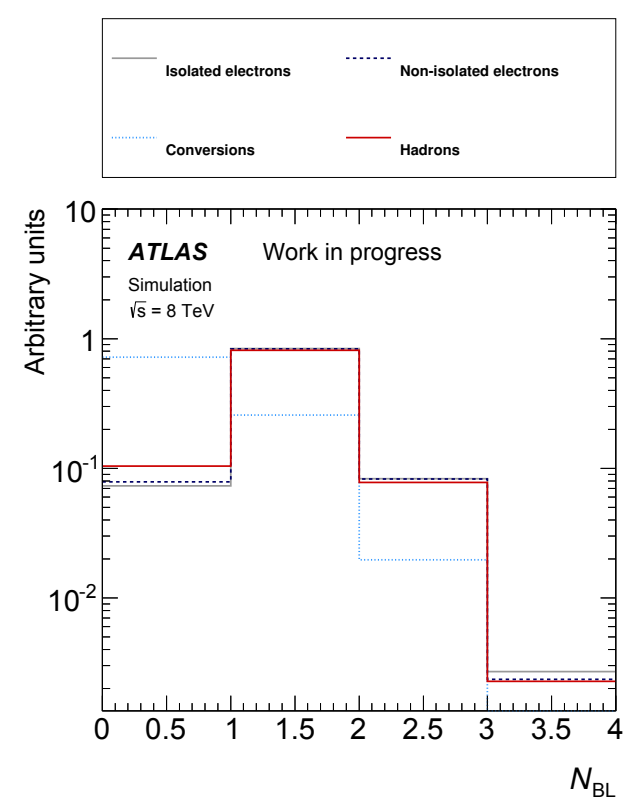

FiguRE 4.19: $N_{\mathrm{BL}}$ distributions of reconstructed electron candidates. The distributions are shown for isolated electrons, as well as the major background components.

The efficiency of the reoptimized version of the MultiLepton menu is compared to that of the original MultiLepton menu in Figure 4.20. The reoptimization resulted in a slight decrease in the average efficiency, from $95 \%$ to $93 \%$. However, it is important to note that there was no significant impact on the dependence of the efficiency on $E_{\mathrm{T}},|\eta|$, or $N_{\mathrm{vtx}}$.

Significant gains in hadron rejection below $40 \mathrm{GeV}$ and in the high material region can be seen in Figure 4.21. Overall, the hadron rejection factor was increased by about $15 \%$. 

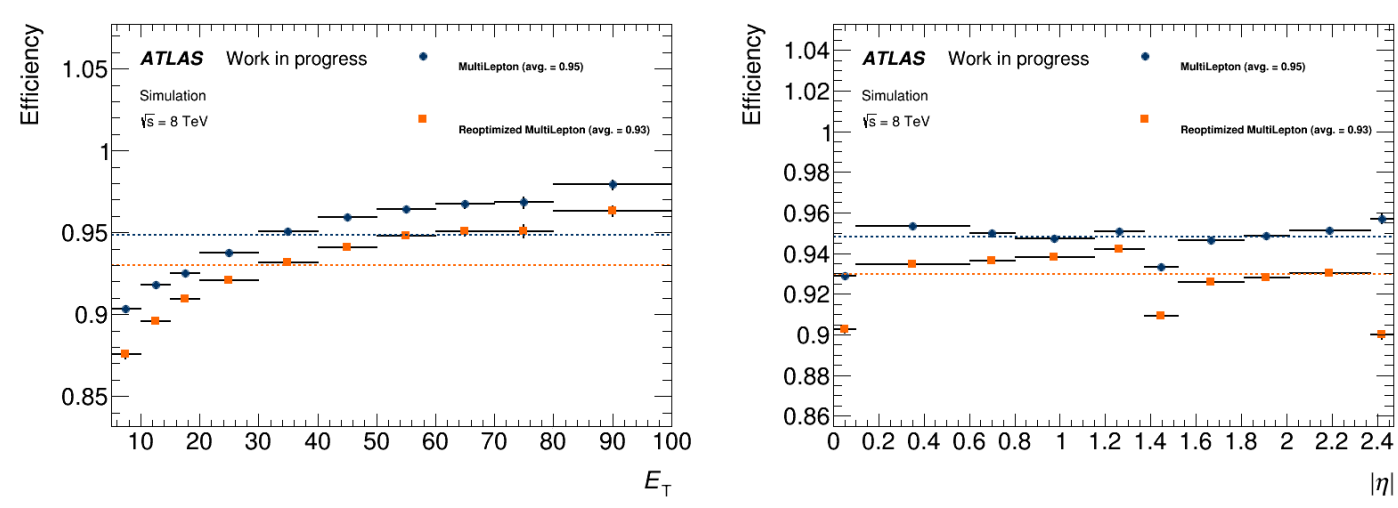

(a) Electron efficiency as a function of $E_{\mathrm{T}}$

(b) Electron efficiency as a function of $|\eta|$

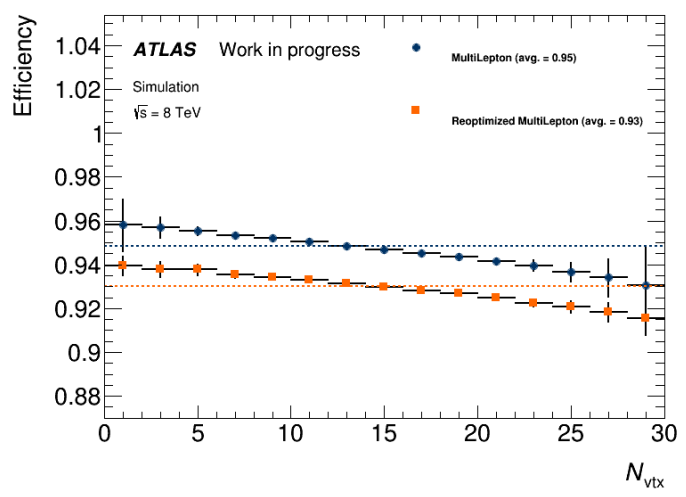

(c) Electron efficiency as a function of $N_{\mathrm{vtx}}$

Figure 4.20: Electron efficiency of the reoptimized MultiLepton menu, compared to the original MultiLepton menu. The efficiencies are shown as a function of $E_{\mathrm{T}},|\eta|$, and $N_{\mathrm{vtx}}$. The dashed lines represent the average efficiency, which corresponds to $93 \%$ for the reoptimized version, and $95 \%$ for the original version.

Finally, the conversion rejection factor was more than doubled, as shown in Figure 4.22. This improvement was largely due to the addition of the B-layer hit requirement. 

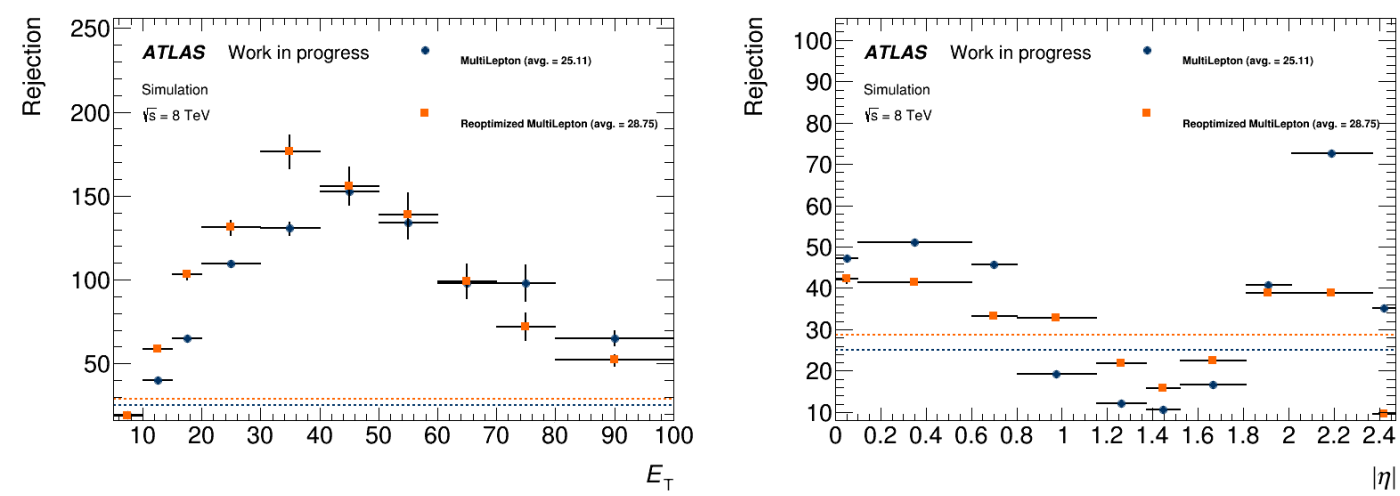

(a) Hadron rejection as a function of $E_{\mathrm{T}}$

(b) Hadron rejection as a function of $|\eta|$

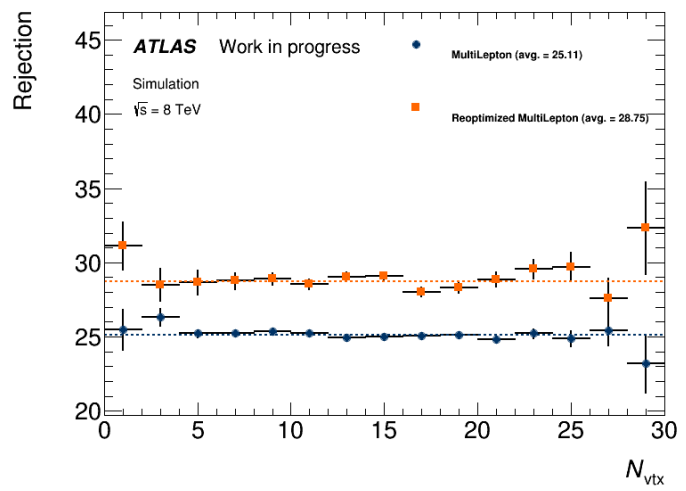

(c) Hadron rejection as a function of $N_{\mathrm{vtx}}$

Figure 4.21: Electron efficiency of the reoptimized MultiLePton menu, compared to the original MultiLepton menu. The dashed lines represent the average rejection, which corresponds to a factor of 28.75 for the reoptimized version, and 25.11 for the original version.

\subsubsection{Summary of the MultiLepton menu}

The MultiLepton offline electron identification menu was designed specifically to meet the demanding requirements of the $H \rightarrow Z Z^{(*)} \rightarrow 4 \ell$ search; it was implemented for the discovery of the Higgs boson [2] and the initial measurements of its properties [5]. The MultiLepton menu consisted of cuts on shower shape, track quality, and track-cluster matching variables, tuned for improved efficiency at low $E_{\mathrm{T}}$ and maximum background rejection, especially in problematic regions 

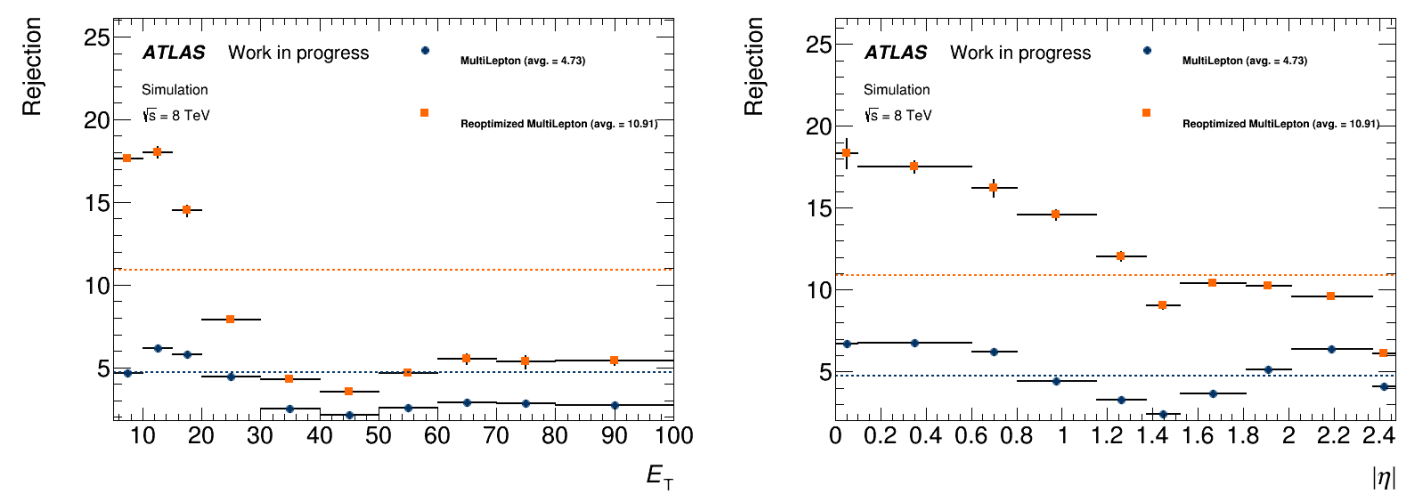

(a) Conversion rejection as a function of $E_{\mathrm{T}}$

(b) Conversion rejection as a function of $|\eta|$

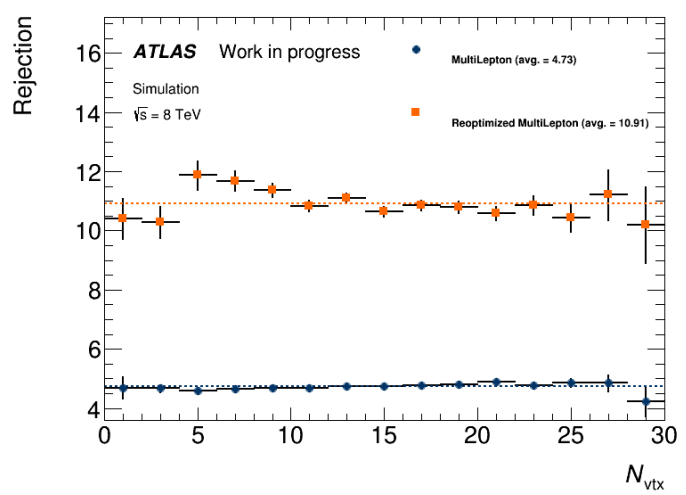

(c) Conversion rejection as a function of $N_{\mathrm{vtx}}$

Figure 4.22: Conversion rejection of the reoptimized MultiLepton menu, compared to the original MultiLepton menu. The dashed lines represent the average rejection, which corresponds to a factor of 10.91 for the reoptimized version, and 4.73 for the original version.

of the detector. The MultiLepton menu featured several variables not included in the standard menu (LoOSE++), and exploited the sensitivity of the new GSF reconstruction algorithm to bremsstrahlung emission. It featured both high signal efficiency and excellent background rejection power and was robust under high pileup conditions; in these respects, the MultiLePton menu's performance exceeded that of the Loose ++ menu. 


\subsection{The likelihood-based method for electron iden- tification}

As the reoptimization of the MultiLepton menu implies, in order to make large gains in background rejection, a certain degree of signal efficiency must be lost; this is the main drawback of cut-based electron identification, since each cut must be applied sequentially, resulting in losses at each step. For this reason, in the final analysis of the $8 \mathrm{TeV}$ Run I data, the MultiLepton menu was superceded by a new, multivariate menu known as the LIKELIHOOD menu [3].

The LiKELIHOOD menu relies on a set of signal and background pdfs (probability density functions; i.e. variable distributions) for a set of $n$ discriminating variables created from data. To make a decision about whether to accept a particular electron having discriminating variable values $x_{i}$, a discriminant $d_{\mathcal{L}}$ is calculated:

$$
d_{\mathcal{L}}=\frac{\mathcal{L}_{S}}{\mathcal{L}_{S}+\mathcal{L}_{B}}
$$

$\mathcal{L}_{S}\left(\mathcal{L}_{B}\right)$ is the signal (background) likelihood, defined as:

$$
\mathcal{L}_{S(B)}(x)=\prod_{i=1}^{n} P_{S(B), i}\left(x_{i}\right),
$$

where $P_{S(B), i}\left(x_{i}\right)$ is the value of the signal (background) PDF of the $i^{\text {th }}$ variable evaluated at $x_{i}$. For the signal, the discriminant peaks at $d_{\mathcal{L}}=1$, while for the background, the discriminant peaks at $d_{\mathcal{L}}=0$. Depending on the degree of efficiency and rejection required, various operating points corresponding to different discriminant values can be chosen. For the purposes of the final $8 \mathrm{TeV}$ 
$H \rightarrow Z Z^{(*)} \rightarrow 4 \ell$ analysis, the Loose LH operating point was chosen; this operating point provides approximately the same efficiency as the MULTiLePton menu, with a background-rejecting factor increased by about $40 \%$.

A multivariate approach to electron identification is advantageous for analyses requiring high levels of efficiency and background rejection, because it considers many variables simultaneously when making a decision, rather than sequentially considering one variable at a time and rejecting objects that fail any one cut. As well, the LIKELIHOOD menu includes several additional discriminating variables that could not be used in the cut-based identification; the signal and background distributions of these variables overlap significantly, making it difficult to extract effective cuts, whereas the LIKELIHOOD algorithm can consider the overall shape of the distributions when calculating the discriminant. Several of the innovations of the MultiLepton menu were incorporated into the Likelihood menu; namely the use of $\Delta p / p$ and $\Delta \phi_{\mathrm{R}}$ as discriminating variables.

Table 4.1 provides a summary of the evolution of offline electron identification in the $H \rightarrow Z Z^{(*)} \rightarrow 4 \ell$ analysis, from the Loose ++ menu used for the $7 \mathrm{TeV}$ data [4], to the MultiLepton menu used for the discovery of the Higgs boson [2] and first measurements of its properties in the $8 \mathrm{TeV}$ data [5], to the Loose LH operating point used for the final analysis of the entire $8 \mathrm{TeV}$ dataset [4].

\subsection{Conclusion}

Offline electron identification is a critical component of the $H \rightarrow Z Z^{(*)} \rightarrow 4 \ell$ analysis. An ideal algorithm selects true signal electrons with a high degree of efficiency, while rejecting the majority of background objects such as hadrons and 


\begin{tabular}{|c|c|c|c|c|}
\hline Variable & Definition & LOOSE ++ & MULTILEPTON & LOOSE LH \\
\hline$E_{\text {ratio }}$ & $\begin{array}{l}\text { Relative difference between the first and } \\
\text { second energy maxima in the strips }\end{array}$ & $\checkmark$ & $\checkmark$ & $\checkmark$ \\
\hline$w_{\mathrm{s}, \text { tot }}$ & Shower width in the strips & $\checkmark$ & $\checkmark$ & \\
\hline$f_{1}$ & Fraction of energy in the strips & & & $\checkmark$ \\
\hline$R_{\eta}$ & $\begin{array}{l}\text { Ratio of energy in } 3 \times 7 / 7 \times 7 \text { cells in } \\
\text { the second EM layer }\end{array}$ & $\checkmark$ & $\checkmark$ & $\checkmark$ \\
\hline$R_{\phi}$ & $\begin{array}{l}\text { Ratio of energy in } 3 \times 3 / 3 \times 7 \text { cells in } \\
\text { the second EM layer }\end{array}$ & & & $\checkmark$ \\
\hline$w_{\eta 2}$ & Shower width in the second EM layer & $\checkmark$ & $\checkmark$ & $\checkmark$ \\
\hline$f_{3}$ & Fraction of energy in the third EM layer & & $\checkmark$ & $\checkmark$ \\
\hline$R_{\mathrm{had}}$ & Hadronic leakage & $\checkmark$ & $\checkmark$ & $\checkmark$ \\
\hline$N_{\mathrm{BL}}$ & Number of B-layer hits & & $\checkmark$ & $\checkmark$ \\
\hline$N_{\text {pix }}$ & Number of pixel hits & $\checkmark$ & $\checkmark$ & $\checkmark$ \\
\hline$N_{\mathrm{Si}}$ & Number of silicon (pixel + SCT) hits & $\checkmark$ & $\checkmark$ & $\checkmark$ \\
\hline$R_{\mathrm{TRT}}$ & Fraction of high-threshold TRT hits & & $\checkmark$ & $\checkmark$ \\
\hline$\Delta p / p$ & Relative momentum loss in the ID & & $\checkmark$ & $\checkmark$ \\
\hline$\Delta \eta_{1}$ & Track-cluster matching in $\eta$ & $\begin{array}{c}\checkmark \\
\text { (flat cut) }\end{array}$ & $\begin{array}{c}\checkmark \\
\text { (binned cuts) }\end{array}$ & $\checkmark$ \\
\hline$\Delta \phi_{\mathrm{R}}$ & Track-cluster matching in $\phi$ & & $\checkmark$ & $\checkmark$ \\
\hline
\end{tabular}

TABle 4.1: Comparison of the variables used in the Loose++, reoptimized Multilepton, and Loose Likelihood menus.

electrons from conversions, and should also be robust under challenging pileup conditions.

In order to accomplish this task, a cut-based menu, known as the MULTILEPTON menu, was developed. This menu relied on a selection of discriminating variables based on shower shape, track quality, and track-cluster matching information, and exploited new features of the GSF electron reconstruction algorithm in order to maximize both the signal efficiency and background rejection. The MultiLepton menu outperformed the standard ATLAS electron identification menu in terms of efficiency at low $E_{\mathrm{T}}$, hadron and conversion rejection, and pileup robustness, and was successfully implemented for the discovery of the Higgs boson and the first measurements of its properties.

Electron identification algorithms must evolve as data-taking conditions and analysis requirements change, and the cut-based MultiLePton menu reached 
the limits of its capabilities as the precision measurements of the Higgs boson's properties required even higher background rejection power without sacrificing efficiency. The MultiLepton menu was retired in favour of a new, multivariate menu, known as the LiKELIHOOD menu, for the final analysis of the $8 \mathrm{TeV}$ data. 


\section{Event selection and background esti- mation}

There is nothing like looking, if you want to find something.

— J. R. R. Tolkien, The Hobbit

in search for the Higgs boson in the four-lepton decay channel, $H \rightarrow$ relatively little background contamination. In this decay channel, the Higgs boson decays immediately to a pair of $Z$ bosons (one of which may be off-shell, denoted by $Z^{*}$; i.e. it does not satisfy the relation $p^{2}=m^{2}$ ). The $Z$ bosons subsequently decay to two pairs of prompt, well-isolated, same-flavour leptons of opposing electric charges, with possible final states: $4 e, 4 \mu, 2 e 2 \mu$, and $2 \mu 2 e$, where in the latter two states, the pairs are ordered in descending order of transverse momentum.

Several background processes may produce similar signals in the detector. These include Standard Model $Z Z^{(*)}$ production, which leads to a four-lepton final state identical to that resulting from Higgs boson decays; this irreducible background is well-understood and is modelled using simulations as described in Section 5.4.1. Other sources of background include $Z+$ jets production, where 
light jets are misidentified as leptons; $Z+b \bar{b}$ production, where the $b$-quark decays produce non-prompt leptons in the final state; and leptonic $t \bar{t}$ decays. Collectively, these make up the reducible background. These processes are not exactly identical to the $H \rightarrow Z Z^{(*)} \rightarrow 4 \ell$ signal; i.e. there may be additional particles present in the final state in the case of jet processes, or the final state may not contain four true leptons, and so the analysis cuts can be tuned to reduce the contribution of these backgrounds as much as possible, as discussed in Section 5.3.4. Methods for estimating the reducible background contribution are discussed in Section 5.4.2 and Chapter 6 .

This chapter will present an overview of the signal and background samples used, as well as the selection used to identify the signal events and reject the backgrounds for the final analysis of the Run I data $[4,6]$.

\subsection{Signal samples}

Simulated signal samples for each of the production modes discussed in Section 2.5.2 have been generated using the POWHEG [73, 74] generator, which calculates separately the matrix elements for gluon-gluon fusion and vector boson fusion production up to next-to-leading order in perturbative QCD. POWHEG is interfaced to Pyтhia8.1 [75, 76] for showering and hadronization, and to Pнотоs [77] for QED corrections. The simulated samples have been reconstructed using the official ATLAS reconstruction software and reweighted to match the pileup profile observed in the data.

The samples are normalized to the theoretical cross-sections, which are summarized in Table 5.1. For the analysis presented in this thesis, the Higgs 


\begin{tabular}{lcc}
\hline \hline$\sigma[\mathrm{pb}]$ & $\sqrt{s}=7 \mathrm{TeV}$ & $\sqrt{s}=8 \mathrm{TeV}$ \\
\hline \hline$g g \rightarrow H$ & $15.1 \pm 1.6$ & $19.3 \pm 2.0$ \\
$q q^{\prime} \rightarrow H q q^{\prime}$ & $1.22 \pm 0.03$ & $1.58 \pm 0.04$ \\
$q \bar{q} \rightarrow W H$ & $0.58 \pm 0.02$ & $0.70 \pm 0.02$ \\
$q \bar{q} \rightarrow Z H$ & $0.34 \pm 0.01$ & $0.42 \pm 0.02$ \\
$g g \rightarrow t \bar{t} H$ & $0.09 \pm 0.01$ & $0.13_{-0.02}^{+0.01}$ \\
\hline$B R\left(H \rightarrow Z Z^{(*)} \rightarrow 4 \ell\right)\left[10^{-3}\right]$ & $0.125 \pm 0.005$ & $0.125 \pm 0.005$ \\
\hline \hline
\end{tabular}

TABLE 5.1: Cross-sections for the production of a Higgs boson of mass $m_{H}=$ $125 \mathrm{GeV}$ in $p p$ collisions at $\sqrt{s}=7 \mathrm{TeV}$ and $8 \mathrm{TeV}$. Cross-sections are given for the gluon-gluon fusion, vector boson fusion and associated production with a $W$ boson, $Z$ boson, and $t \bar{t}$ production modes. The branching ratio $(B R)$ for the $H \rightarrow Z Z^{(*)} \rightarrow 4 \ell$ decay mode is also given [78].

boson production cross-sections and decay branching ratios, and their uncertainties, are taken from $[78,79]$ and are given in Table 5.1. For the gluon-gluon fusion production mode, $g g \rightarrow H$, the cross-sections have been calculated to next-to-next-to-leading order [80-85] in QCD. As well, QCD soft-gluon resummations calculated in the next-to-next-to-leading log approximation are applied to the gluon-gluon fusion process [86]. Next-to-leading order electroweak radiative corrections are also applied [87, 88]. For the vector boson fusion production mode, $q q^{\prime} \rightarrow H q q^{\prime}$, the cross-sections are calculated with full next-to-leading order QCD and electroweak corrections [89-91]. The cross-sections for the associated $W H / Z H$ production modes are calculated at next-to-next-to-leading order [92, 93] in QCD, with next-to-leading order radiative corrections [94]. The cross-sections for the $t \bar{t} H$ mode are estimated up to next-to-leading order QCD [95-99]. The branching ratio of the Higgs boson decay [100] to the four-lepton final state is predicted by PROPHECY4F [101, 102], including next-to-leading order QCD and electroweak corrections, as well as the effects of interference between identical final-state leptons. 


\subsection{Background samples}

For the $Z Z^{(*)}$ background, Powheg [103] is used to generate events produced via quark-antiquark annihilation, while GG2ZZ [104] is used to model the gluongluon fusion production mechanism. The $Z+$ jets background is modelled using Alpgen [105] interfaced to Pythia8.1 for hadronization and showering, including $Z+c \bar{c}$ in the massless approximation and $Z+b \bar{b}$ from parton showers. The $Z+b \bar{b}$ background is also modelled using AlPGEn interfaced to PythiA8.1, but with the $b$-quark mass taken into account using matrix element calculations. The $t \bar{t}$ background is modelled using Powheg interfaced to PYTHIA8.1 for parton shower hadronization and PHOTOs for QED corrections, and the $W Z$ background is modelled using SHERPA [106].

\subsection{Event selection}

\subsubsection{Data quality}

To ensure the validity of the results, the dataset used in the analysis consists only of events recorded during periods when stable beams had been declared in the LHC and all detector subsystems were operating nominally. The dataset used for this analysis corresponds to an integrated luminosity of $4.5 \mathrm{fb}^{-1}$ recorded at centre-ofmass energies of $\sqrt{s}=7 \mathrm{TeV}$ in 2011 and $20.3 \mathrm{fb}^{-1}$ recorded at $\sqrt{s}=8 \mathrm{TeV}$ in 2012. 


\subsubsection{Trigger}

In order to pass the preselection stage of the analysis, events must pass a singlelepton or dilepton trigger: these correspond to single-electron or dielectron triggers in the case of $4 e$ events and single-muon or dimuon triggers for $4 \mu$ events, while $2 \mu 2 e$ and $2 e 2 \mu$ events must pass any of the aforementioned triggers or an electronmuon trigger. As the LHC's instantaneous luminosity and pileup increased while data-taking progressed from 2011 to 2012 , the minimum $p_{\mathrm{T}}$ thresholds were raised in order to rule out low- $p_{\mathrm{T}}$ particles originating from pileup and maintain a reasonable trigger rate. In 2012, an isolation requirement was added to the lowest- $p_{\mathrm{T}}$ single-lepton trigger: the sum of the $p_{\mathrm{T}}$ of all tracks in a cone of $\Delta R<0.2$ around the lepton, excluding the lepton itself, must not exceed $10 \%$ of the lepton's $p_{\mathrm{T}}$. Table 5.2 summarizes the trigger thresholds used for the $7 \mathrm{TeV}$ and $8 \mathrm{TeV}$ analyses. To compensate for differences in the trigger efficiencies observed in data and Monte Carlo, the simulated events are reweighted by applying a lepton trigger efficiency scale factor [107].

\begin{tabular}{ccc}
\hline \hline Trigger & \multicolumn{2}{c}{ Threshold $[\mathrm{GeV}]$} \\
& $\sqrt{s}=7 \mathrm{TeV}(2011)$ & $\sqrt{s}=8 \mathrm{TeV}(2012)$ \\
\hline Single-electron & $20-22$ & $24 i$ or 60 \\
Single-muon & 18 & $24 i$ or 36 \\
Dielectron & $12 / 12$ & $12 / 12$ \\
Dimuon & $10 / 10$ & $13 / 13$ or $18 / 8$ \\
Electron-muon & $10 / 6$ & $12 / 8$ or $24 i / 8$ \\
\hline \hline
\end{tabular}

TABLE 5.2: Trigger $p_{\mathrm{T}}$ thresholds $[\mathrm{GeV}]$ used in the $7 \mathrm{TeV}$ and $8 \mathrm{TeV}$ analyses. In the case of the electron-muon trigger, the first number refers to the electron threshold and the second refers to the muon threshold. Note that the singleelectron trigger threshold rose from $20 \mathrm{GeV}$ to $22 \mathrm{GeV}$ over the course of the 2011 data-taking period. The symbol " $i$ " denotes an isolated lepton trigger. 


\subsubsection{Object selection}

The following describes the procedures used to select leptons produced in Higgs boson decays as well as jets, which are signatures of certain Higgs boson production mechanisms. Table 5.3 summarizes the selection procedure.

\begin{tabular}{lccc}
\hline \hline Object & Algorithm / identification & $p_{\mathrm{T}}[\mathrm{GeV}]$ & $|\eta|$ \\
\hline Electron & Loose $++(7 \mathrm{TeV}) /$ LoOsE LH $(8 \mathrm{TeV})$ & $>7$ & $<2.47$ \\
Muon & STACO CB/ST & $>6$ & $<2.7$ \\
& Stand-alone & $>6$ & $2.5<|\eta|<2.7$ \\
& CaloTag & $>15$ & $<0.1$ \\
Jet & Anti- $k_{\mathrm{T}}, R=0.4$ & $>25$ & $<2.4$ \\
& & $>30$ & $2.4<|\eta|<4.5$ \\
\hline \hline
\end{tabular}

TABLE 5.3: Selection criteria for electrons, muons, and jets used in the $H \rightarrow$ $Z Z^{(*)} \rightarrow 4 \ell$ analysis.

\subsubsection{Electron selection}

Electron candidates must have a well-reconstructed track refitted with the GSF algorithm and matched with an EM calorimeter cluster. To ensure this, all candidates must pass offline identification, as described in Chapter 4. The identification requirements have changed from 2011 to 2012; this was necessitated by the increasingly challenging data-taking conditions as well as the demands of the analysis from the search for and discovery of the Higgs boson through to the first precision measurements of its properties. For the final analysis presented here, the Loose ++ menu (refer to Section 4.2) was used for the 2011 data, and the Loose LH menu (refer to Section 4.4) was used for the 2012 data.

The electron energy calibration applied to the data, as well as the simulated signal and irreducible $Z Z$ background samples, is performed using multivariate algorithms, taking into account the most up-to-date knowledge of the detector 
geometry [108]. The most up-to-date simulated reducible background samples available correspond to a previous version of the detector geometry and are calibrated accordingly. In order to improve the energy resolution of electrons having low $p_{\mathrm{T}}$ or falling within problematic regions of the detector, a so-called $E-p$ combination is applied. For electrons with $p_{\mathrm{T}}<30 \mathrm{GeV}$ and $|\eta|<1.52$, and having track $p_{\mathrm{T}}$ and cluster energy values $\left(E_{\mathrm{Cl}}\right)$ consistent with one another, a maximum likelihood fit is used to combine the cluster energy and track $p_{\mathrm{T}}$; this combined value is then used to construct the electron's four-momentum. This procedure reduces the width of the four-lepton invariant mass distribution by approximately $4 \%[6]$.

Finally, electron candidates must satisfy the following kinematic requirements: they must be identified within the cluster pseudorapidity region $|\eta|_{\mathrm{Cl}}<$ 2.47 and the transverse energy, defined as $E_{\mathrm{T}}=E_{\mathrm{Cl}} / \cosh \left(\eta_{\text {Track }}\right)$, must be at least $7 \mathrm{GeV}$.

For simulated events, reconstruction and identification efficiency scale factors are applied to each electron candidate in order to take into account observed differences in efficiencies between data and Monte Carlo [3].

\subsubsection{Muon selection}

Muon candidates are reconstructed using the STACO algorithm, as described in Chapter 3. All four reconstruction categories (combined, segment-tagged, calorimetertagged, and stand-alone) are accepted, with a maximum of one stand-alone or one calorimeter-tagged muon permitted per event. 
The track quality requirements differ for the 2011 and 2012 data; this reflects updates in the muon reconstruction software. These selection criteria are outlined in Table 5.4 .

\begin{tabular}{lcc}
\hline \hline & $\sqrt{s}=7 \mathrm{TeV}(2011)$ & \\
\hline B-layer hits & $\geq 1$, if expected \\
Pixel hits + crossed dead pixel sensors & $>1$ \\
SCT hits + crossed dead SCT sensors & $>5$ \\
Pixel holes + number of SCT holes & $<3$ \\
TRT hits $\left(N_{\text {Hits }}\right)$ and outliers $\left(N_{\text {Outliers }}\right)$ & $|\eta|<1.9: N_{\text {Hits }}+N_{\text {Outliers }}>5$ AND $\frac{N_{\text {Outliers }}}{N_{\text {Hits }}+N_{\text {Outliers }}<0.9}$ \\
& $|\eta| \geq 1.9:$ IF $N_{\text {Hits }}+N_{\text {Outliers }}>5, \frac{N_{\text {Outliers }}}{N_{\text {Hits }}+N_{\text {Outliers }}}<0.9$ \\
\hline \hline B-layer hits & $\sqrt{s}=8 \mathrm{TeV}(2012)$ & - \\
Pixel hits + crossed dead pixel sensors & $>0$ \\
SCT hits + crossed dead SCT sensors & $>4$ \\
Pixel holes + number of SCT holes & $<.1<|\eta| \leq 1.9: N_{\text {Hits }}+N_{\text {Outliers }}>5$ AND $\frac{N_{\text {Outliers }}}{N_{\text {Hits }}+N_{\text {Outliers }}}<0.9$ \\
TRT hits $\left(N_{\text {Hits }}\right)$ and outliers $\left(N_{\text {Outliers }}\right)$ & 0.1 \\
\hline \hline
\end{tabular}

TABLE 5.4: Muon track quality requirements for the $7 \mathrm{TeV}$ and $8 \mathrm{TeV}$ analyses.

In order to satisfy kinematic requirements, combined and segment-tagged muons must have $p_{\mathrm{T}}>6 \mathrm{GeV}$ and $|\eta|<2.7$, calorimeter-tagged muons must have $p_{\mathrm{T}}>15 \mathrm{GeV}$ and $|\eta|<0.1$, and stand-alone muons must have $p_{\mathrm{T}}>6 \mathrm{GeV}$ and $2.5<|\eta|<2.7$.

Simulated muons are reweighted to data using reconstruction efficiency scale factors; in addition, the transverse momentum is corrected in order to improve the muon momentum scale [109].

\subsubsection{Jet selection}

Jets are reconstructed from topological clusters using the anti- $k_{\mathrm{T}}$ algorithm with distance parameter $R=0.4$, as described in Section 3.3.4. The jet energy scale used to correct the topoclusters is determined from simulation for the $7 \mathrm{TeV}$ data $[110]$ and from data for the $8 \mathrm{TeV}$ data. Jets resulting from pileup are removed by requiring that the jet vertex fraction, defined as the fraction of tracks 
originating from the primary vertex, be greater than $75 \%$ (50\%) for jets having $p_{\mathrm{T}}<50 \mathrm{GeV}$ and $|\eta|<2.4$ in the $7 \mathrm{TeV}(8 \mathrm{TeV})$ data.

The kinematic requirements are as follows: jets in the region $|\eta|<2.4$ must have $p_{\mathrm{T}}>25 \mathrm{GeV}$, while in the region $2.4<|\eta|<4.5$, jets must have $p_{\mathrm{T}}>30 \mathrm{GeV}$.

\subsubsection{Removal of overlapping objects}

After the object selection cuts, overlap removal is performed to ensure that no duplicate objects reconstructed by multiple algorithms are kept, and to reduce ambiguity due to overlapping clusters. If two EM calorimeter clusters share the same inner detector track, the electron object having the highest $E_{\mathrm{T}}$ is kept. Electron candidates sharing the same track as a combined or segment-tagged muon are rejected. In the case of a calorimeter-tagged muon, however, the muon candidate is rejected and the electron candidate is retained. Jets overlapping with electrons within $\Delta R<0.2\left(\right.$ where $\left.\Delta R=\sqrt{\Delta \eta^{2}+\Delta \phi^{2}}\right)$ are removed.

\subsubsection{Quadruplet formation and Higgs boson candidate se- lection}

Events with at least four electrons, four muons, or two electrons and two muons passing object selection are then used to construct quadruplets, which are then subject to additional selection criteria. The leptons must form two same-flavour, opposite-sign pairs, and the three highest- $p_{\mathrm{T}}$ leptons must satisfy $p_{\mathrm{T}}>20,15$, $10 \mathrm{GeV}$. At least one (two) leptons must match the single-lepton (dilepton) trigger that fired for the event. Only one quadruplet is permitted per event; the leading 
dilepton $\left(Z_{1}\right)$ is chosen by taking the lepton pairing having an invariant mass closest to the nominal $Z$ boson mass. In the case of more than four leptons in an event, the subleading dilepton $\left(Z_{2}\right)$ is then chosen based on the pairing that gives the next closest mass to the nominal value.

Quadruplets must pass the following selection cuts, which have been chosen to optimize the signal sensitivity:

- Leading $\mathbf{Z}$ boson mass $\left(\mathbf{m}_{\mathbf{1 2}}\right)$ The leading $Z$ boson must have an invariant mass in the range $50 \mathrm{GeV}<m_{12}<106 \mathrm{GeV}$.

- Subleading $\mathbf{Z}$ boson mass $\left(\mathbf{m}_{\mathbf{3 4}}\right)$ The invariant mass of the subleading $Z$ boson mass must fall within the range $m_{\text {threshold }}<m_{34}<115 \mathrm{GeV}$, where $m_{\text {threshold }}$ is in the range $12 \mathrm{GeV}<m_{\text {threshold }}<50 \mathrm{GeV}$. The value of $m_{\text {threshold }}$ is dependent on the four-lepton invariant mass, $m_{4 \ell}$ and is determined by linear interpolation. The mass values used for the interpolation are given in Table 5.5 .

\begin{tabular}{ccccc}
\hline \hline$m_{4 \ell}[\mathrm{GeV}]$ & $<140$ & 140 & 190 & $>190$ \\
$m_{\text {threshold }}[\mathrm{GeV}]$ & 12 & 12 & 50 & 50 \\
\hline \hline
\end{tabular}

TABLE 5.5: Mass points used to interpolate the minimum threshold for the $m_{34}$ cut: $m_{\text {threshold }}<m_{34}<115 \mathrm{GeV}$.

- Separation in $\Delta \mathbf{R}$ Leptons from Higgs boson decays must be well-separated. Same-flavour (opposite-flavour) leptons must satisfy $\Delta R\left(\ell_{i}, \ell_{j}\right)>0.1(0.2)$.

- $\mathbf{J} / \psi$ veto To avoid the possibility of mispaired leptons coming from $J / \psi$ decays $\left(m_{J / \psi}=3.1 \mathrm{GeV}\right)$, the cross-paired leptons from $4 e$ and $4 \mu$ events are checked. If a dilepton with an invariant mass $m_{\ell \ell}<5 \mathrm{GeV}$ can be constructed using one lepton from each of the $Z$ bosons, the event is rejected. 
- Track isolation discriminant $\left(\Sigma \mathbf{p}_{\mathbf{T}} / \mathbf{p}_{\mathbf{T}}\right)$ Leptons produced in Higgs boson decays must be well-isolated, so isolation cuts are applied to the leptons in order to reduce the background due to jets. To quantify the isolation of the lepton track in the inner detector, the sum of the $p_{\mathrm{T}}$ of all tracks in a cone of size $\Delta R<0.2$ around the lepton, excluding the lepton itself, is required to be less than $15 \%$ of the lepton's $p_{\mathrm{T}}$. Each lepton in the quadruplet must satisfy this requirement.

- Calorimeter isolation discriminant $\left(\Sigma \mathbf{E}_{\mathbf{T}} / \mathbf{p}_{\mathbf{T}}\right)$ Similarly, the calorimeter clusters associated with the leptons must also be well-isolated. For electrons in the $7 \mathrm{TeV}(8 \mathrm{TeV})$ analysis, the sum of the $E_{\mathrm{T}}$ within a cone of size $\Delta R<0.2$ around the electron (excluding the electron itself) must be less than $30 \%(20 \%)$ of the electron's $E_{\mathrm{T}}$. The difference in the requirements for the two datasets is due to the summed $E_{\mathrm{T}}$ being calculated using individual calorimeter cells (topological clusters) in the $7 \mathrm{TeV}(8 \mathrm{TeV})$ analysis. For muons, the sum of the $E_{\mathrm{T}}$ within a cone of size $\Delta R<0.2$ around the muon, excluding the muon itself, must be less than $30 \%$ of the muon's $p_{\mathrm{T}}$. Each lepton in the quadruplet must satisfy this requirement.

- Impact parameter significance $\left(\mathbf{d}_{\mathbf{0}} / \sigma_{\mathbf{d}_{\mathbf{0}}}\right)$ To exclude leptons from heavy quark decays, which tend to be associated with vertices displaced from the primary vertex, a cut is made on the significance of the transverse impact parameter, i.e. the distance of closest approach of the track to the primary vertex, measured in the transverse plane. For all electrons (muons) in the quadruplet, it is required that the ratio of the impact parameter to its uncertainty satisfy $d_{0} / \sigma_{d_{0}}<6.5(3.5)$. Since this parameter is affected by the emission of bremsstrahlung, a higher limit is permitted for electrons. 
Events passing the full selection are considered to be candidate $H \rightarrow$ $Z Z^{(*)} \rightarrow 4 \ell$ events.

\subsubsection{Candidate event corrections}

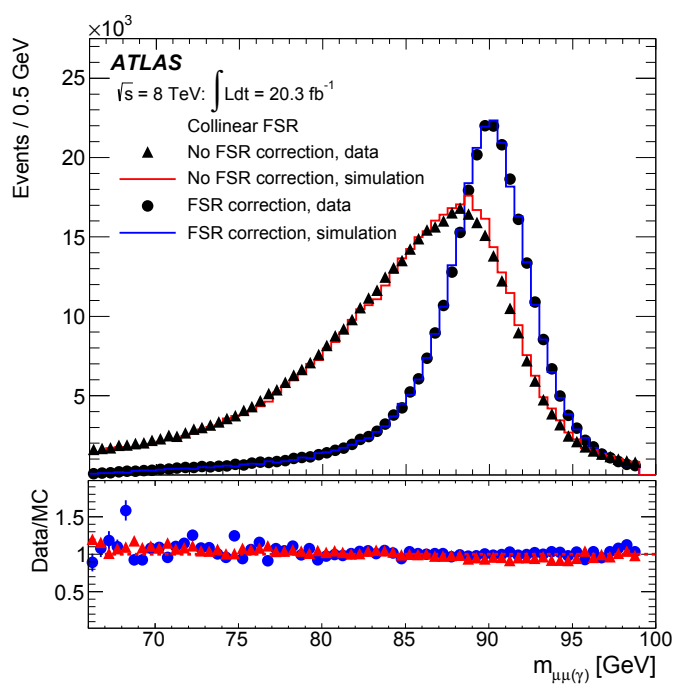

(a) Collinear FSR

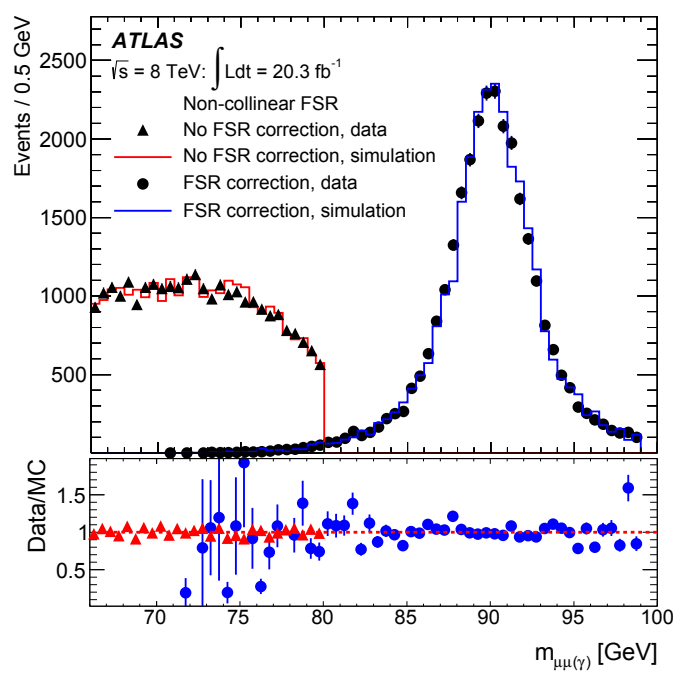

(b) Non-collinear FSR

FiguRE 5.1: The effects of collinear and non-collinear FSR recovery on the $Z$ boson invariant mass distribution, using $Z \rightarrow \mu \mu$ decays in data and simulation $[6]$.

Additional corrections are applied to candidates in order to improve the invariant mass resolution. These include:

- Final state radiation (FSR) recovery A method has been developed to recover final state radiation photons emitted by muons and electrons [6]. If the leading $Z$ boson decays to a dimuon with an invariant mass in the range $66 \mathrm{GeV}<m_{12}<89 \mathrm{GeV}$, the algorithm searches first for collinear photons emitted within a cone of $\Delta R<0.15$ around the muon. If the leading $Z$ boson decays to a dielectron, or if no collinear photons are found, noncollinear photons are then considered, provided that the subleading dilepton 
(which may be either a dimuon or a dielectron in this case) has a mass of $m_{34}<81 \mathrm{GeV}$. At most, the contribution of one FSR photon is permitted to be added to the four-momentum of the four-lepton system, as long as the invariant dilepton-photon mass $m_{\ell \ell \gamma}<100 \mathrm{GeV}$. The recovery of FSR photons has a notable effect on the $Z$ boson invariant mass resolution, as shown in Figure 5.1.

- $\mathbf{Z}$ mass constraint In order to improve the invariant mass resolution, a $Z$ mass constraint is applied [6]. This procedure consists of maximizing the likelihood defined as the product of the $Z$ line shape and the momentum response functions of the two leading leptons, in order to constrain the leading dilepton mass, $m_{12}$. In the low-mass case, where $m_{4 \ell}<190 \mathrm{GeV}$, the subleading dilepton mass is also constrained. As a result, the four-lepton invariant mass resolution is improved by approximately $15 \%$.

\subsection{Background estimation}

\subsubsection{Irreducible $Z Z^{(*)}$ background}

The dominant source of background is the irreducible $Z Z^{(*)}$ background, in which two $Z$ bosons are produced directly in proton-proton collisions and subsequently decay to four leptons. As shown in Figure 5.2, the final state of this process is identical to that of the $H \rightarrow Z Z^{(*)} \rightarrow 4 \ell$ process and so they are indistinguishable in the detector; hence, the irreducible $Z Z^{(*)}$ background's contribution to the observed events cannot be reduced by tuning the analysis cuts. Since $Z$ boson decays are well-understood, this process is modelled using simulations. 


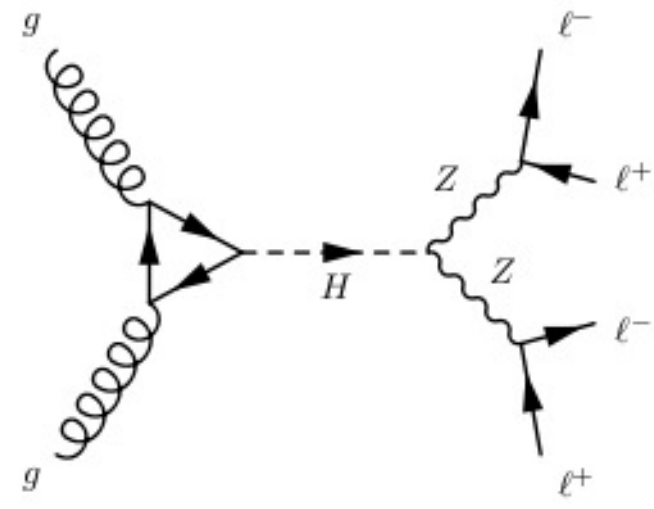

(a) $g g \rightarrow H \rightarrow Z Z^{(*)} \rightarrow 4 \ell$

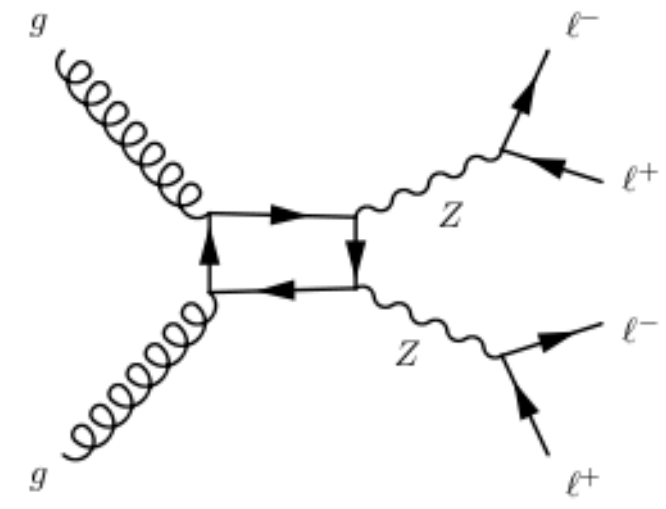

(b) $g g \rightarrow Z Z^{(*)} \rightarrow 4 \ell$

Figure 5.2: Comparison of the $H \rightarrow Z Z^{(*)} \rightarrow 4 \ell$ signal and irreducible $Z Z^{(*)} \rightarrow 4 \ell$ background final states.

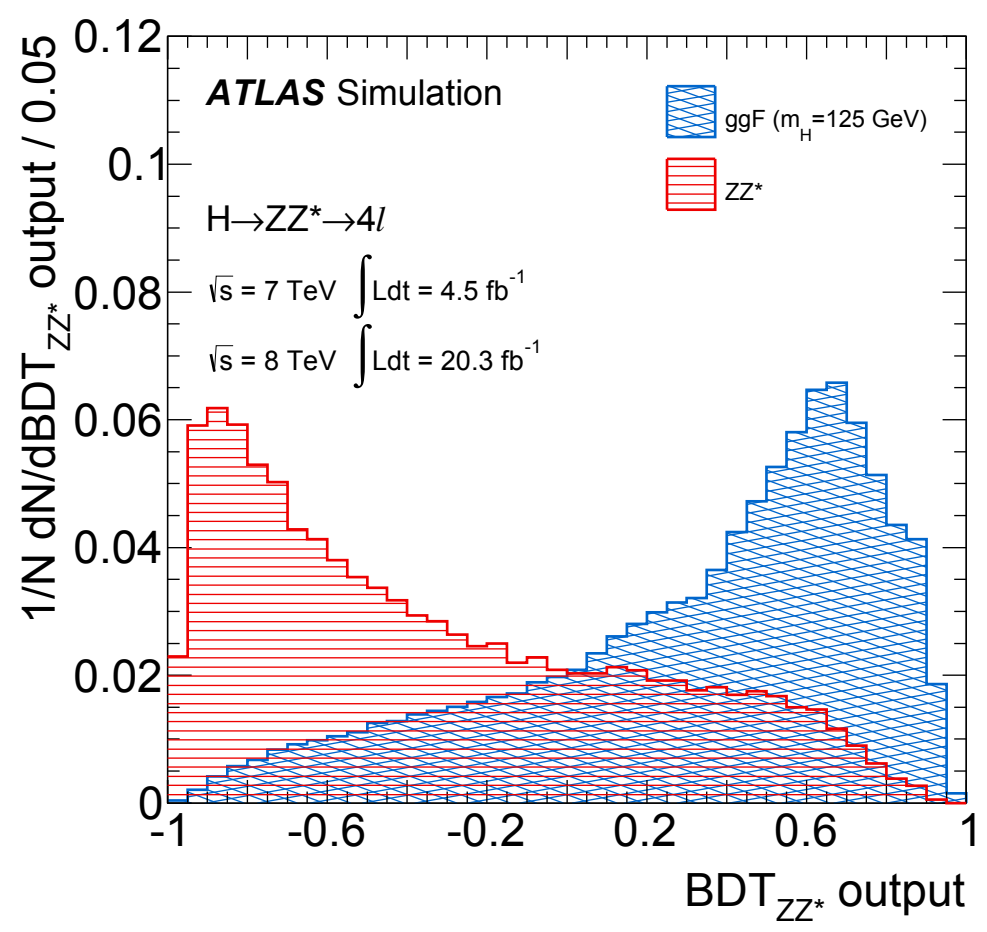

Figure 5.3: Example BDT output scores for simulated signal and $Z Z^{(*)}$ events [6]. 
A multivariate analysis tool, known as a boosted decision tree (BDT) [111], is used to discriminate between signal and $Z Z^{(*)}$ events based on their kinematic properties for the purpose of improving the Higgs boson mass measurement. Simulated signal events with $m_{H}=125 \mathrm{GeV}$ and simulated $Z Z^{(*)}$ events in the mass range $115 \mathrm{GeV}<m_{Z Z^{(*)}}<130 \mathrm{GeV}$ are used to train the BDT; the input values include the four-lepton system's $p_{\mathrm{T}}$ and $\eta$, as well as a matrix element-based kinematic discriminant. The information "learned" by the tool about each of these variables during the training is then used to decide whether a given event observed in data is more likely to belong to the signal or the background. This decision is made in the form of an output BDT score, which is designed to peak towards 1 for signal events and towards -1 for $Z Z^{(*)}$ events, as illustrated in Figure 5.3.

\subsubsection{Reducible background}

Several other processes contribute to what is known as the reducible background, which can be minimized using carefully tuned selection cuts. The yield of the reducible background, which is not as well-understood as the irreducible background and hence not as easily simulated, is estimated using data-driven methods. The reducible background components, illustrated in Figure 5.4, include:

- $\mathbf{Z}+$ jets In this case, a $Z$ boson is accompanied by light quark jets which are misidentified as leptons.

- $\mathbf{Z}+\mathbf{b} \overline{\mathbf{b}}$ Here, a $Z$ boson is produced in association with heavy (i.e. $b$ or $c$ ) quarks, which then emit leptons as they decay via the weak interaction.

- $\mathbf{t} \overline{\mathbf{t}}$ In this process, a $t \bar{t}$ pair decays via the weak interaction, eventually resulting in the emission of four leptons. 
- WZ This process involves the production of a $Z$ boson, which then decays to a lepton pair, in addition to a $W$ boson, which decays to a lepton and a neutrino. If an additional lepton is present or an accompanying jet is misidentified as a lepton, a second dilepton may be misidentified.

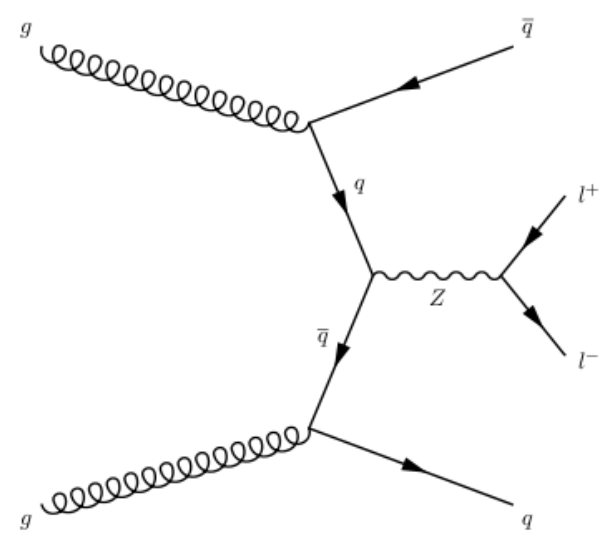

(a) $Z+$ jets

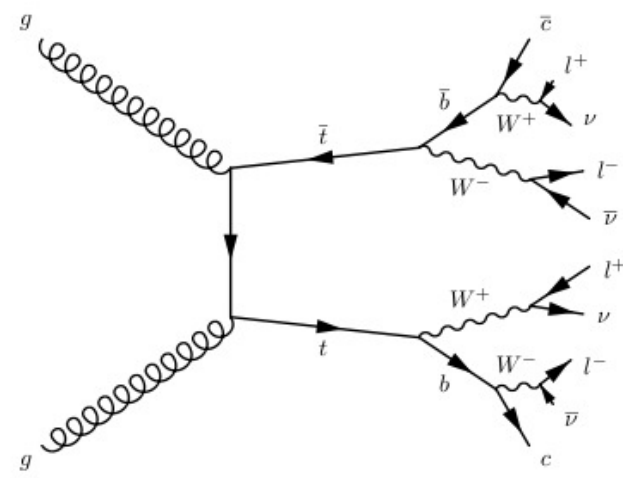

(c) $t \bar{t}$

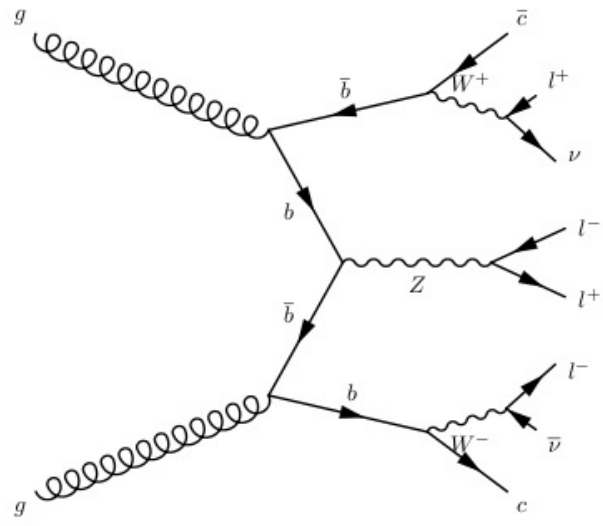

(b) $Z+b \bar{b}$

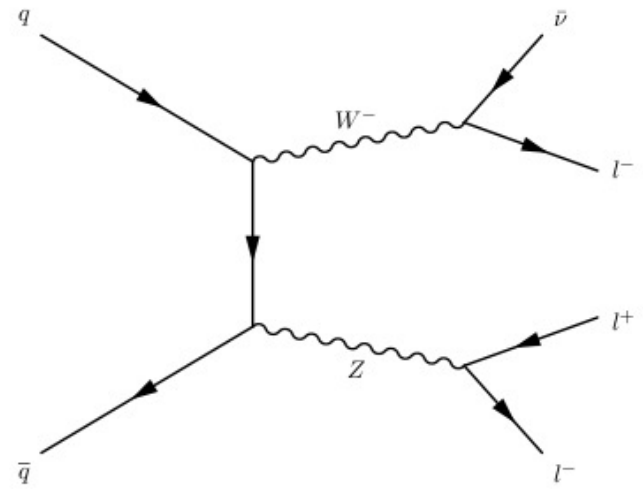

(d) $W Z$

Figure 5.4: Example Feynman diagrams for the main sources of reducible background to the $H \rightarrow Z Z^{(*)} \rightarrow 4 \ell$ signal.

The background composition varies depending on the flavour of the lower$p_{\mathrm{T}}$ subleading leptons; hence, different methods are used to estimate the reducible backgrounds for the subleading ee and $\mu \mu$ channels. Each case relies on data-driven methods following the same basic approach: a control region, orthogonal to the signal region, is constructed by inverting or relaxing background-rejecting selection 
cuts. The probabilities for the background components to pass the selection cuts are determined using a signal-free sample, and then these probabilities are used to extrapolate the background yield in the signal region from the control region. Several methods are used to provide a reliable estimate for each channel; the following provides a brief description of the baseline methods. An alternative method for the estimation of the subleading ee background, developed by the author of this thesis, will be discussed in detail in Chapter 6 .

\subsubsection{Reducible $Z+\mu \mu$ background estimation}

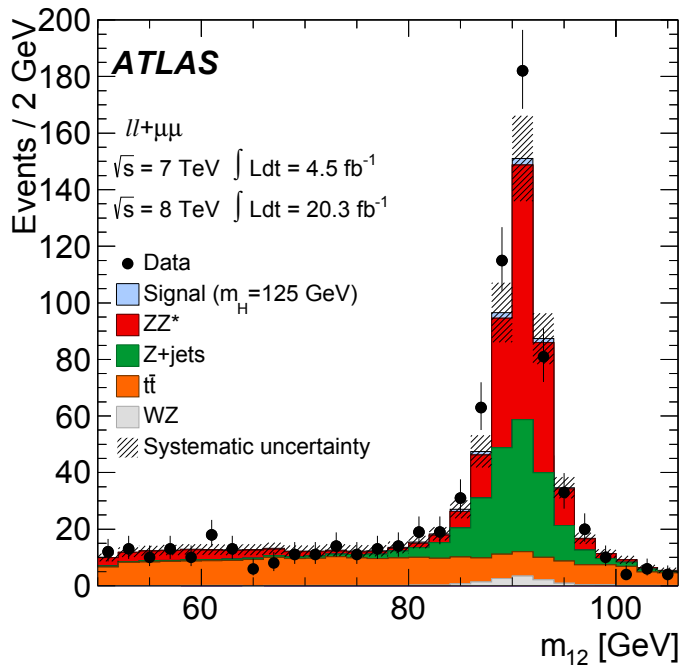

(a) $m_{12}$

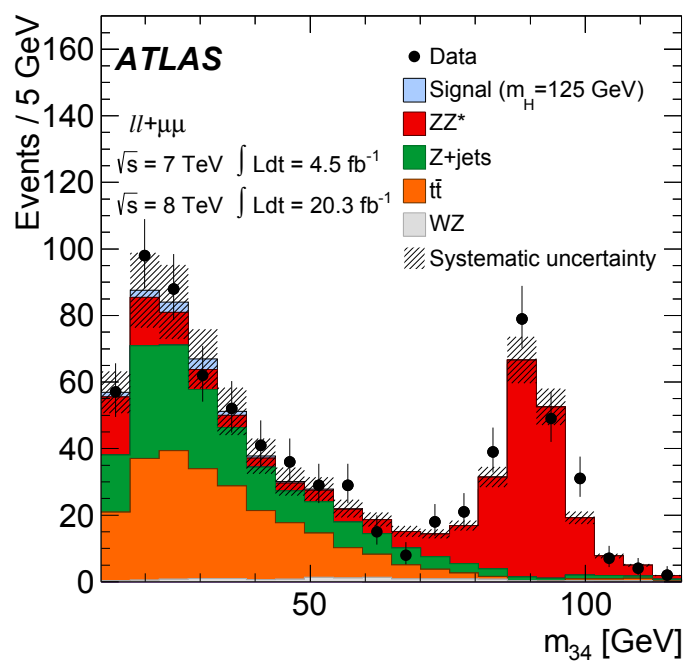

(b) $m_{34}$

FiguRE 5.5: Invariant mass distributions of the leading and subleading dileptons in the control region consisting of a leading $Z$ boson passing the full analysis requirements, plus a subleading muon pair with the isolation and impact parameter significance requirements relaxed. The signal, irreducible $Z Z^{(*)}$ background, and reducible background contributions are shown [6].

As shown in Figure 5.5, the reducible muon-like background in the $4 \mu$ and $2 e 2 \mu$ channels is dominated by the $t \bar{t}$ and $Z+b \bar{b}$ processes, with a small $Z+$ jets contribution from $\pi / K$ in-flight decays. The contributions of the background 
components are determined by exploiting differences in the shapes of the $m_{12}$ distributions of each component: the $t \bar{t}$ distribution is broad, while the distribution in channels involving $Z$ boson production peak at the $Z$ boson mass. The background contributions are determined by constructing four separate control regions enriched in the various background sources and simultaneously fitting the $m_{12}$ distributions.

The four control regions used in this method are:

- Inverted $\mathbf{d}_{0}$ significance control region Events must have a $Z_{1}$ passing the standard analysis cuts plus two subleading reconstructed muons passing all cuts with the exception of the impact parameter significance cut, which must be failed by at least one muon. This control region is enriched in heavy flavour decays; i.e. $Z+b \bar{b}$ and $t \bar{t}$.

- Inverted isolation control region Here, the $Z_{1}$ passes the standard analysis cuts, while at least one of the subleading muons must fail the isolation requirement. Requiring that the subleading muons pass the impact parameter significance cut removes the heavy flavour component, while enriching the control region in $Z+$ jets.

- Same-sign control region This control region is equally enriched in all background components. The leading $Z$ boson must pass all selection cuts, while the subleading muons are required to have the same sign and the isolation and impact parameter significance cuts are relaxed.

- $\mathbf{e} \mu+\mu \mu$ control region This control region is enriched in $t \bar{t}$ by requiring a different-flavour, opposite-sign leading dilepton passing the standard cuts and a subleading pair of reconstructed muons with no charge, isolation, or impact parameter significance requirements applied. 


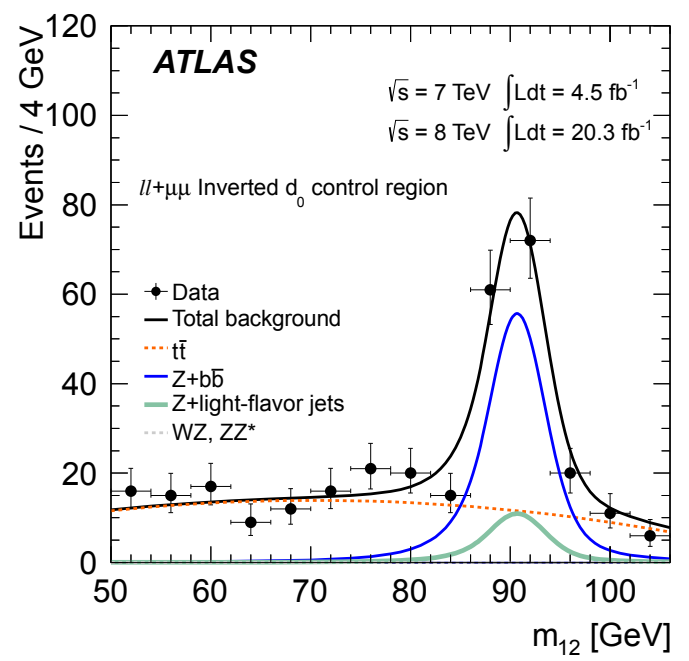

(a) Inverted $d_{0}$ significance control region

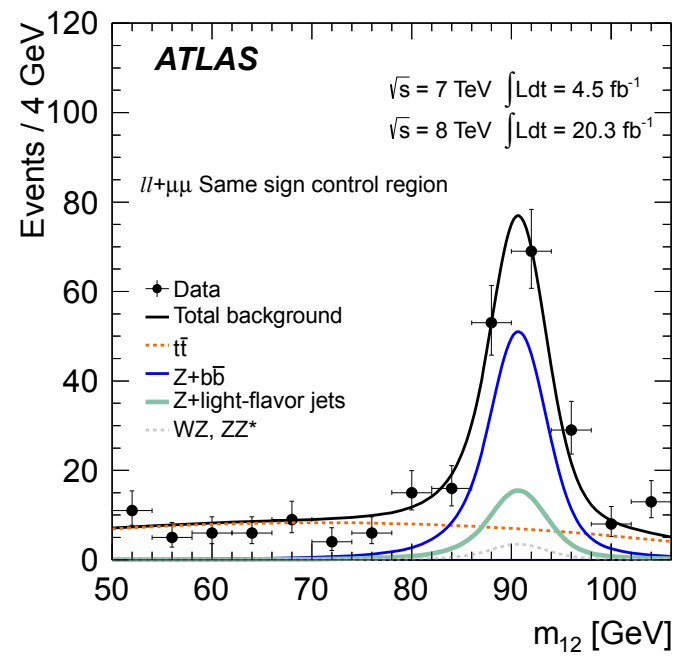

(c) Same-sign control region

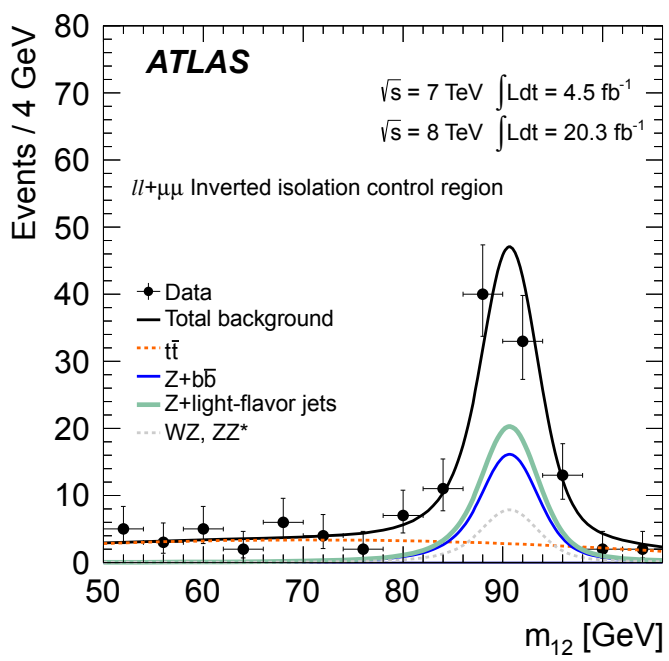

(b) Inverted isolation control region

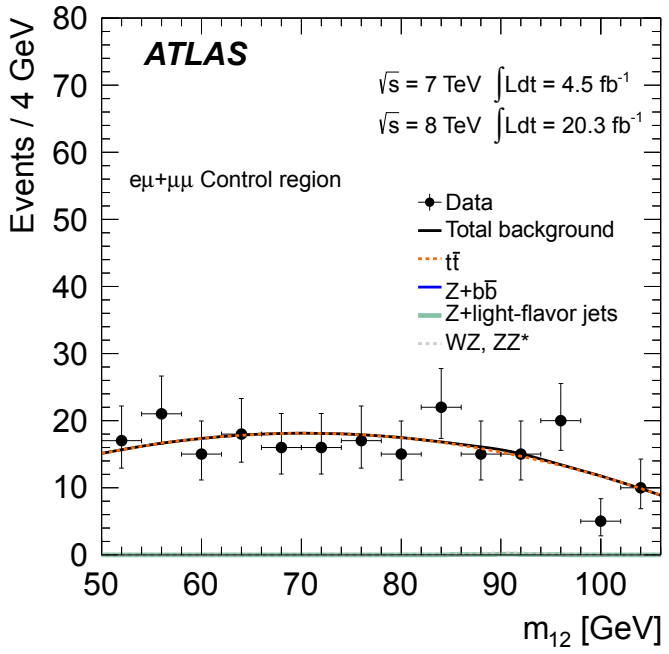

(d) $e \mu+\mu \mu$ control region

FiguRE 5.6: Observed leading dimuon invariant mass distributions and results of the maximum likelihood fits in the four control regions used to estimate the reducible muon-like background [6].

A simultaneous maximum likelihood fit to the $m_{12}$ distribution is used to extract the number of background events in the control regions as shown in Figure 5.6. This is then extrapolated to the signal region using transfer factors based on the efficiencies of the selection cuts in order to obtain the final $Z+\mu \mu$ background estimate; the resulting background yields are presented in Table 5.6 
for the $7 \mathrm{TeV}$ and $8 \mathrm{TeV}$ analyses. The statistical and systematic uncertainties correspond to the uncertainties of the fit and the transfer factors, respectively.

\begin{tabular}{|c|c|c|}
\hline Component & $4 \mu$ & $2 e 2 \mu$ \\
\hline \multicolumn{3}{|c|}{$\sqrt{s}=7 \mathrm{TeV}$} \\
\hline$Z+b \bar{b}$ & $0.36 \pm 0.19 \pm 0.07$ & $0.25 \pm 0.13 \pm 0.05$ \\
\hline$Z+$ jets & $0.06 \pm 0.08 \pm 0.04$ & $0.04 \pm 0.06 \pm 0.02$ \\
\hline$t \bar{t}$ & $0.081 \pm 0.016 \pm 0.021$ & $0.056 \pm 0.011 \pm 0.015$ \\
\hline$W Z$ & $0.08 \pm 0.05$ & $0.19 \pm 0.10$ \\
\hline \multicolumn{3}{|c|}{$\sqrt{s}=8 \mathrm{TeV}$} \\
\hline$Z+b \bar{b}$ & $2.30 \pm 0.26 \pm 0.14$ & $2.01 \pm 0.23 \pm 0.13$ \\
\hline$Z+$ jets & $0.81 \pm 0.38 \pm 0.41$ & $0.57 \pm 0.31 \pm 0.41$ \\
\hline$t \bar{t}$ & $0.51 \pm 0.03 \pm 0.09$ & $0.48 \pm 0.03 \pm 0.08$ \\
\hline$W Z$ & $0.42 \pm 0.07$ & $0.44 \pm 0.06$ \\
\hline
\end{tabular}

TABLE 5.6: Estimated reducible $Z+\mu \mu$ background yield (in number of events) in the $7 \mathrm{TeV}$ and $8 \mathrm{TeV}$ data. The errors refer to the statistical and systematic uncertainties, respectively.

\subsubsection{Reducible $Z+e e$ background estimation}

The reducible electron-like background in the $4 e$ and $2 \mu 2 e$ channels is dominated by $Z+$ jets processes in which light hadrons are misidentified as electrons ("fakes", denoted by " $f$ "), as shown in Figure 5.7. Additional contributions come from heavy quark decays (" $q$ ") and photon conversions (" $\gamma$ ").

The baseline method used to extract the $Z+e e$ background is known as the $3 \ell+X$ method. A control region containing four leptons, three of which must pass the the full selection cuts (i.e. a $Z$ boson plus an additional lepton) is defined. The fourth lepton, which has the lowest $p_{\mathrm{T}}$ of the two subleading leptons, is only required to pass basic track quality cuts: more than six silicon tracker hits and more than one hit in the pixel detector. Electron identification, isolation, and 


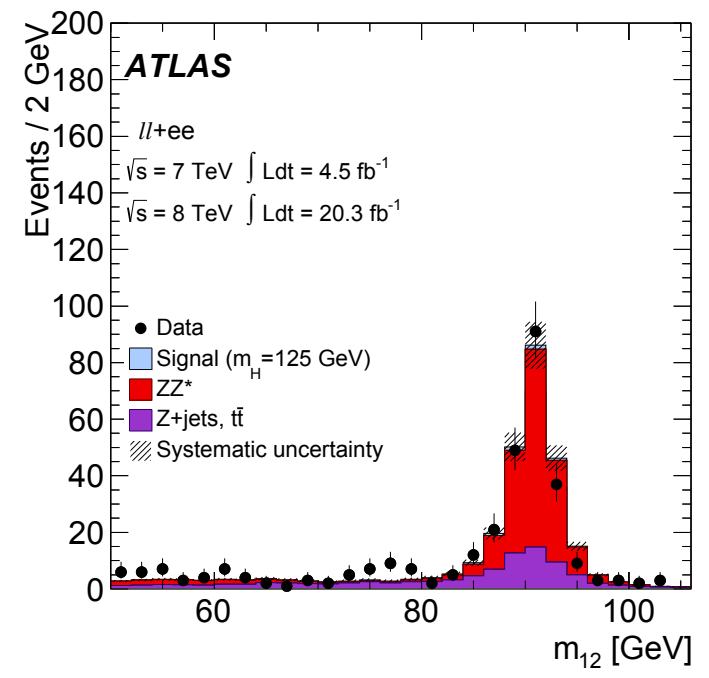

(a) $m_{12}$

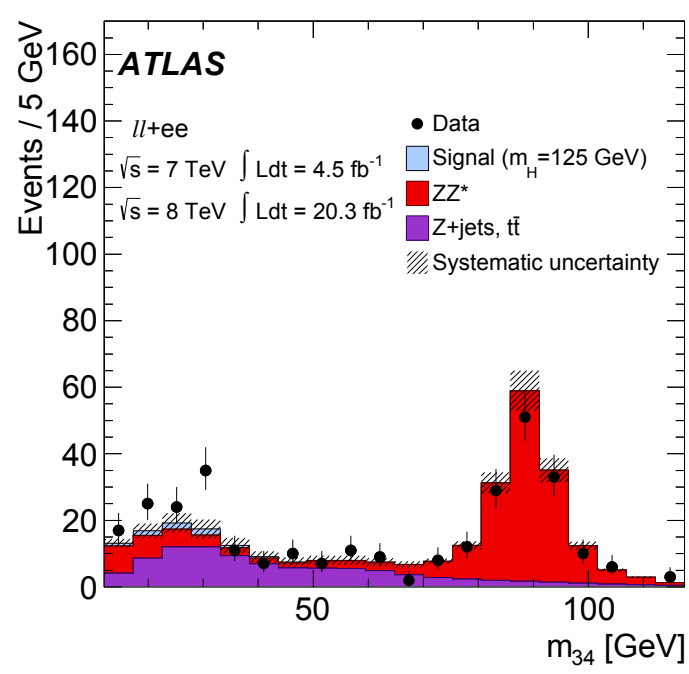

(b) $m_{34}$

FiguRE 5.7: Invariant mass distributions of the leading and subleading dileptons in the control region consisting of a leading $Z$ boson passing the full analysis requirements, plus a subleading electron pair with the isolation and impact parameter significance requirements relaxed. The signal, irreducible $Z Z^{(*)}$ background, and reducible background contributions are shown [6].

impact parameter cuts are not applied. The two subleading leptons are required to have the same sign in order to suppress the $Z Z^{(*)}$ contribution.

The contribution of each background type is estimated by simultaneously fitting the distributions of several discriminating variables: the number of B-layer hits (which is typically 0 for photons), and the ratio of the sum of high-threshold hits and outliers in the TRT to the sum of low-threshold hits and outliers, which is typically lower $\left(R_{\mathrm{TRT}}<0.1\right)$ for hadrons than for electrons. The fit results are shown in Figure 5.8.

To extrapolate the background yields to the signal region, efficiencies for each background component to pass the identification, isolation, and impact parameter significance cuts are extracted from simulations and corrected to take into account differences between data and simulation. The procedure for the efficiency 


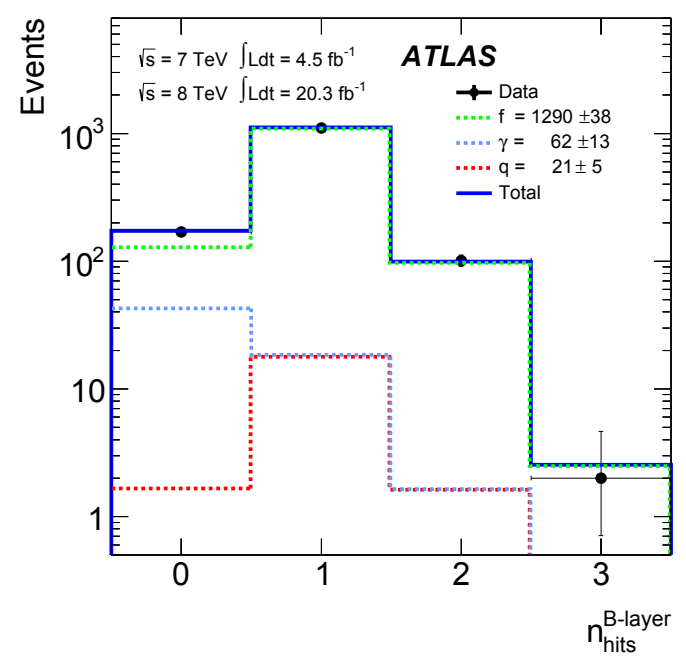

(a) Number of B-layer hits

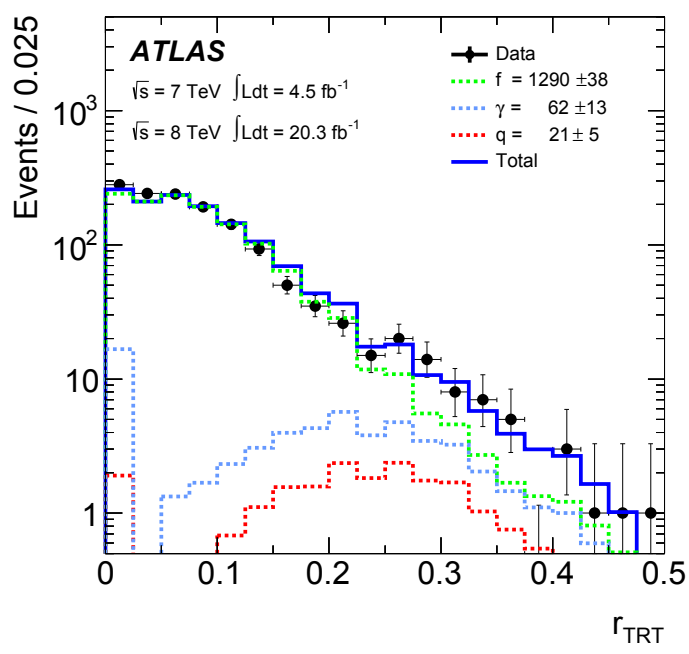

(b) TRT high-threshold hits ratio

FIgURE 5.8: Observed distributions of the number of B-layer hits and the TRT high-threshold hits ratio in the $3 \ell+X$ control region used to estimate the reducible muon-like background. The results of simultaneous fits for the fake $(f)$, heavy quark decays $(q)$, and photon conversion $(\gamma)$ background components are also shown $[6]$.

calculation and correction is similar to the one used for the method described in Chapter 6, and will be described in detail therein.

Table 5.7 gives the estimated $Z+e e$ background yields in the signal region for the $7 \mathrm{TeV}$ and $8 \mathrm{TeV}$ analyses. The effect of the LikeLiHood electron identification can be seen by comparing the background yields in the two datasets; the yields are approximately equal despite the $8 \mathrm{TeV}$ dataset being approximately four times larger than the $7 \mathrm{TeV}$ dataset. A residual contribution from $Z Z^{(*)}$ is determined from simulations and subtracted to obtain the final reducible background estimate. The systematic uncertainties include the uncertainty of the fit, and the uncertainty of the transfer factors. 


\begin{tabular}{|c|c|c|}
\hline Component & $4 e$ & $2 \mu 2 e$ \\
\hline \multicolumn{3}{|c|}{$\sqrt{s}=7 \mathrm{TeV}$} \\
\hline$f$ & $1.91 \pm 0.14 \pm 0.40$ & $2.02 \pm 0.15 \pm 0.42$ \\
\hline$q$ & $0.23 \pm 0.05 \pm 0.04$ & $0.28 \pm 0.06 \pm 0.06$ \\
\hline$\gamma$ & $1.24 \pm 0.47 \pm 0.26$ & $0.75 \pm 0.44 \pm 0.16$ \\
\hline$Z Z^{(*)}$ & $0.08 \pm 0.01$ & $0.10 \pm 0.01$ \\
\hline Final estimate & $3.3 \pm 0.5 \pm 0.5$ & $2.9 \pm 0.5 \pm 0.5$ \\
\hline \multicolumn{3}{|c|}{$\sqrt{s}=8 \mathrm{TeV}$} \\
\hline$f$ & $1.45_{-0.07}^{+0.07}{ }_{-0.47}^{+0.47}$ & $1.65_{-0.08}^{+0.08}{ }_{-0.53}^{+0.53}$ \\
\hline$q$ & $0.83_{-0.18}^{+0.18}{ }_{-0.14}^{+0.14}$ & $0.96_{-0.26}^{+0.26}{ }_{-0.16}^{+0.16}$ \\
\hline$\gamma$ & $0.68_{-0.20}^{+0.20}{ }_{-0.20}^{+0.20}$ & $0.43_{-0.19-0.21}^{+0.19+0.21}$ \\
\hline$Z Z^{(*)}$ & $0.08 \pm 0.01$ & $0.13 \pm 0.01$ \\
\hline Final estimate & $2.88 \pm 0.28 \pm 0.54$ & $2.91 \pm 0.33 \pm 0.60$ \\
\hline
\end{tabular}

TABLE 5.7: Reducible $Z+e e$ background yield (in number of events) due to hadrons faking electrons ("f"), heavy quark decays ("q"), and photon conversions ( " $\gamma$ ") in the $7 \mathrm{TeV}$ and $8 \mathrm{TeV}$ data determined using the $3 \ell+X$ method. The final estimate is obtained by summing the yields of these components and subtracting the residual $Z Z^{(*)}$ contribution determined from simulations. The errors refer to the statistical and systematic uncertainties, respectively.

\subsection{Conclusion}

The selection procedure for the $H \rightarrow Z Z^{(*)} \rightarrow 4 \ell$ analysis, using both data and simulation, as well as the baseline methods used to determine the background yields, have been outlined. Before looking at the final results of the analysis, the next chapter will explore in detail an alternative method developed by the author of this thesis for estimating the reducible electron-like background. The final results of the event selection will be presented in Chapter 7 . 


\section{A data-driven estimation of the reducible electron-like background in the $H \rightarrow$ $Z Z^{(*)} \rightarrow 4 \ell$ channel: the Transfer Fac- tor method}

And then! Oh, the noise! Oh, the Noise! Noise! Noise! Noise!

— Dr. Seuss, How the Grinch Stole Christmas

S discussed in Chapter 5 , the main source of reducible background in the by light jets originating from light quarks or gluons and misidentified as electrons $(Z+$ jets $)$. Additional background sources include the production of a $Z$ boson accompanied by electrons resulting from heavy quark decays $(Z+b \bar{b}), t \bar{t}$ decays, and $W Z$ decays.

In the case of the $Z+$ jets background, this complex process is currently not understood well enough to be able to accurately model its composition, as well as 
6. A data-driven estimation of the reducible electron-like background in the $H \rightarrow Z Z^{(*)} \rightarrow 4 \ell$ channel: the Transfer Factor method

the rate at which hadrons are misidentified in the detector. Hence, it was chosen to estimate the reducible electron-like background using data-driven methods.

One such method is the "Transfer Factor" method, which was developed by the author of this thesis in order to cross-check the baseline method described in Section 5.4.2 [4, 6]. A control region enriched in background is constructed from data by relaxing or inverting the selection cuts designed to reject background (e.g. electron identification, isolation, and impact parameter signficance, as described in Section 5.3). The probability for background objects to pass the cuts is determined using a signal-free sample of electron candidates. The transfer factor is then defined as the ratio of the probability for a given event in the control region to pass the selection (i.e. for it to be assigned to the signal region) to the probability for it to fail the selection (as is expected of the control region). The yield in the control region, $N_{\mathrm{CR}}$, is then scaled by this factor to extrapolate the background yield in the signal region. The transfer factor, $T F$, is determined for each event in the control region; summing the transfer factors then yields the estimated background yield in the signal region, $N_{\mathrm{SR}}$ :

$$
N_{\mathrm{SR}}=\sum_{i=1}^{N_{\mathrm{CR}}} T F_{i}=\sum_{i=1}^{N_{\mathrm{CR}}} \frac{P_{i}(\text { pass })}{P_{i}(\text { fail })}
$$

The categorization of the electron-like background is described in Section 6.1. Section 6.2 describes the selection of the control region, Section 6.3 describes the determination of the efficiencies for background objects, and the final results for the estimation of the background yield in the signal region are presented in Section 6.5. Following the validation of the method for the $8 \mathrm{TeV}$ analysis, it was adapted for use with the $7 \mathrm{TeV}$ dataset; the results are presented in Section 6.6. 
6. A data-driven estimation of the reducible electron-like background in the $H \rightarrow Z Z^{(*)} \rightarrow 4 \ell$ channel: the Transfer Factor method

\subsection{Categorization of the electron-like background}

The electron-like background can be separated into various categories, based on the behaviour of the particles in the detector (i.e. their track and shower shape properties), and the processes from which they originated. Since these factors influence the probability that a reconstructed electron object will pass the selection cuts, the cut efficiencies and transfer factors are calculated separately for each category. Objects may be categorized based on their properties as reconstructed in the detector; simulated objects may be additionally categorized based on Monte Carlo truth information.

\subsubsection{Truth categories}

Electron-like objects may be separated into four general categories, based on the process from which they originate. When working with simulated events, the background objects may be classified into these categories using generator-level (truth) information about a reconstructed particle's type and origin (i.e. its parent particle). These categories are defined as follows:

- True, isolated electrons (e) These are true, isolated, prompt electrons produced mainly in decays of $W$ and $Z$ bosons.

- Electrons from heavy quark decays (q) While these are also true electrons, they originate mainly from $b$-quark decays and thus have tracks originating from displaced vertices. Unlike electrons in the $e$ category, they are not isolated. 
6. A data-driven estimation of the reducible electron-like background in the $H \rightarrow Z Z^{(*)} \rightarrow 4 \ell$ channel: the Transfer Factor method

- Photons $(\gamma)$ These consist mainly of electrons originating from photon conversions; in addition, final state radiation (FSR) photons misidentified as electrons are included in this category.

- Fakes (f) Everything else not falling into one of the previous categories is considered to be a fake. In practice, these objects are predominantly hadrons misidentified as electrons.

\subsubsection{Reconstruction categories}

Reconstructed electron objects may be categorized as electron-like or fake-like, according to the following criteria. Each reconstruction category contains a mixture of the four truth categories defined in Section 6.1.1; the proportion of each truth category varies between reconstruction categories.

- Electron-like (E) Electron-like objects are categorized based on shower shape and tracking information. They must satisfy the following criteria to reduce the contributions of fakes and photons, respectively: at least $10 \%$ of the cluster energy must be deposited in the first layer of the EM calorimeter $\left(f_{1}>0.1\right)$, and a B-layer hit must occur, if expected (if a track crosses a dead region of the B-layer, a hit is assumed to occur). In the region covered by the TRT $(|\eta|<2.0)$, an $\eta$-dependent cut on the TRT ratio (defined in Section 4.1.2) is applied. Elsewhere, electron-like objects are distinguished from fakes using a cut on the ratio of energy using the ratio of energy deposits in clusters of $\Delta \eta \times \Delta \phi=3 \times 3$ and $3 \times 7$ EM calorimeter cells (where a cell is defined in terms of the second layer of the EM calorimeter and measures $\Delta \eta \times \Delta \phi=0.025 \times 0.025): R_{\phi}>0.9$. 
6. A data-driven estimation of the reducible electron-like background in the $H \rightarrow Z Z^{(*)} \rightarrow 4 \ell$ channel: the Transfer Factor method

- Fake-like (F) All objects not falling into the electron-like category are classified as fake-like.

Table 6.1 summarizes the prescription for separating electron objects into reconstruction categories.

\begin{tabular}{cc}
\hline \hline Electron-like & Fake-like \\
$f_{1}>0.1$ & Everything else \\
$N_{\mathrm{BL}}>0$ (if expected) & \\
$|\eta|<2.0:$ & $\eta$-dependent $R_{\mathrm{TRT}}$ cut \\
$|\eta| \geq 2.0: R_{\phi}>0.9$ & \\
\hline \hline
\end{tabular}

TABLE 6.1: Electron reconstruction category selection criteria.

\subsection{The $Z+X X$ control region}

The control region for the background estimation is chosen such that contributions from the $H \rightarrow Z Z^{(*)} \rightarrow 4 \ell$ signal and irreducible $Z Z^{(*)}$ background are suppressed. It is made up of quadruplets of reconstructed leptons, with the leading pair being the decay products of a $Z$ boson as selected in the main analysis, and the subleading pair consisting of two reconstructed electron objects, denoted by $X_{3}$ and $X_{4}$. This control region is hence known as the $Z+X X$ control region.

For the subleading $X$ objects, the basic electron selection requirements described in Section 5.3.3.1 are applied, with the exception of electron identification, which is designed to reject the background objects that are desired in this case. Instead, a relaxed version of the identification menu designed specifically for background studies is used. Known as the LOOSERELAXED LH operating point, this menu is designed to accept a factor of roughly 7.5 times more background objects than the standard Loose LH operating point described in Section 4.4. 
6. A data-driven estimation of the reducible electron-like background in the $H \rightarrow Z Z^{(*)} \rightarrow 4 \ell$ channel: the Transfer Factor method

The control region is designed to be signal-free by inverting at least one of the electron identification, track isolation, calorimeter isolation, or impact parameter significance cuts as defined in Section 5.3.3.1 for both of the $X$; i.e. both must fail at least one of these cuts (note that both $X$ need not fail the same cut). These requirements also effectively eliminate the contribution from $Z Z^{(*)}$ events.

Table 6.2 gives the yield of this selection when applied to the $8 \mathrm{TeV}$ data, as well as a detailed breakdown of the expected yields from each of the irreducible background processes obtained by performing the same selection on the background Monte Carlo samples described in Section 5.2. The $X$ are assigned to reconstruction categories as described in Section 6.1.2, and the events are classified as $E E, E F, F E$, or $F F$, where the higher- $p_{\mathrm{T}} X$ (i.e. $X_{3}$ ) is categorized first. The yields for each category are given in Table 6.3. The excess of events in data with respect to the Monte Carlo samples is due to the different electron energy calibrations applied to the data and the reducible background simulations, as described in Section 5.3.3.1. These events form the control region from which the estimated number of background events in the signal region is extrapolated.

Using the logic of Equation 6.1, the transfer factor used to extrapolate from this control region to the signal region is defined as:

$$
T F=\frac{\epsilon_{3} \cdot \epsilon_{4}}{\left(1-\epsilon_{3}\right) \cdot\left(1-\epsilon_{4}\right)}
$$

where $\epsilon_{3}$ and $\epsilon_{4}$ are the probabilities for each of the $X$ to pass the selection cuts; i.e. the efficiencies of those cuts when applied on background objects. The numerator of the transfer factor represents the probability for both $X$ in the event to pass the selection cuts and be assigned to the signal region, while the denominator 
6. A data-driven estimation of the reducible electron-like background in the $H \rightarrow Z Z^{(*)} \rightarrow 4 \ell$ channel: the Transfer Factor method

represents the probability for both $X$ in the event to fail (which defines the control region).

\begin{tabular}{ccc}
\hline \hline & $4 e$ & $2 \mu 2 e$ \\
\hline Data & $653.0 \pm 25.6$ & $706.0 \pm 26.6$ \\
\hline$Z+$ jets & $448.1 \pm 15.9$ & $552.0 \pm 19.8$ \\
$Z+b \bar{b}$ & $49.7 \pm 1.3$ & $56.9 \pm 1.4$ \\
$t \bar{t}$ & $52.8 \pm 0.9$ & $65.0 \pm 1.0$ \\
$W Z$ & $2.3 \pm 0.2$ & $2.3 \pm 0.2$ \\
Total & $552.9 \pm 19.0$ & $676.2 \pm 22.4$ \\
\hline \hline
\end{tabular}

TABLE 6.2: Observed and expected yields in the $Z+X X$ control region in the $8 \mathrm{TeV}$ data and Monte Carlo, with $\mathcal{L}=20.3 \mathrm{fb}^{-1}$. The errors given correspond to the statistical uncertainty.

\begin{tabular}{ccc}
\hline \hline Category & $4 e$ & \multicolumn{1}{c}{$2 \mu 2 e$} \\
\hline$E E$ & $100 \pm 10$ & $99 \pm 10$ \\
$E F$ & $191 \pm 14$ & $208 \pm 14$ \\
$F E$ & $117 \pm 11$ & $131 \pm 12$ \\
$F F$ & $245 \pm 16$ & $268 \pm 16$ \\
\hline \hline
\end{tabular}

TABle 6.3: Composition of the $Z+X X$ control region in terms of the number of events observed in each of the electron-like and fake-like reconstruction categories, as observed in the $8 \mathrm{TeV}$ data, with $\mathcal{L}=20.3 \mathrm{fb}^{-1}$. The errors correspond to the statistical uncertainty.

\subsection{Selection efficiency of electron-like background objects}

\subsubsection{The $Z+X$ control sample}

In order to determine the efficiencies for background objects to pass the selection cuts, a signal-free control sample enriched in electron-like background objects is 
6. A data-driven estimation of the reducible electron-like background in the $H \rightarrow Z Z^{(*)} \rightarrow 4 \ell$ channel: the Transfer Factor method

selected, consisting of on-shell $Z$ boson decays accompanied by a single reconstructed electron object, denoted by " $X$." The choice of $Z+X$ events, comprising exactly three reconstructed leptons (three electrons or two muons and one electron) passing the lepton selection cuts outlined in Section 5.3.3.1 and Section 5.3.3.2, ensures the absence of contamination in the form of true, isolated electrons from both the $H \rightarrow Z Z^{(*)} \rightarrow 4 \ell$ signal and the $Z Z^{(*)}$ background. To reduce the presence of true, isolated electrons from $W Z$ decays, which are characterized by large amounts of missing transverse energy, the event must satisfy the requirement $E_{\mathrm{T}}^{\text {miss }}<50 \mathrm{GeV}$.

The $Z$ boson is permitted to decay to either two muons or two electrons, and must follow the same selection cuts as the $Z_{1}$ in the main analysis, as described in Section 5.3, with the following exceptions: both leptons must have $p_{\mathrm{T}}>20 \mathrm{GeV}$, and the invariant mass must lie within $15 \mathrm{GeV}$ of the nominal $Z$ boson mass, in order to ensure the cleanest possible sample of $Z$ boson decays.

\begin{tabular}{ccc}
\hline \hline Events & Data & MC \\
\hline$Z+X$ & $254811 \pm 504$ & $264440 \pm 514$ \\
$Z+E$ & $80192 \pm 283$ & $83705 \pm 289$ \\
$Z+F$ & $174619 \pm 418$ & $180735 \pm 425$ \\
\hline \hline
\end{tabular}

TABLE 6.4: $Z+X$ event yields in the $8 \mathrm{TeV}$ data and from simulation, with $\mathcal{L}=$ $20.3 \mathrm{fb}^{-1}$. The global event yield is subdivided into reconstruction categories, in which the $X$ may be either electron-like $(E)$ or fake-like $(F)$. The errors correspond to the statistical uncertainty.

The electron identification requirement is relaxed for the $X$, which must pass the LOOSERELAXED LH selection, while the isolation and impact parameter significance cuts are not applied. 
6. A data-driven estimation of the reducible electron-like background in the $H \rightarrow Z Z^{(*)} \rightarrow 4 \ell$ channel: the Transfer Factor method

Table 6.4 gives the number of $Z+X$ events obtained from the $8 \mathrm{TeV}$ dataset, as well as the corresponding yield from simulations. The $Z+X$ yields are assigned to reconstruction categories as defined in Section 6.1.2.

The $Z+X$ sample is then used to determine the efficiencies, i.e. the probabilities for an electron-like background object $X$ to pass the electron identification, track isolation, calorimeter isolation, and impact parameter selection cuts as defined in Section 5.3. The efficiency is defined simply as the fraction of events in the sample passing the cuts:

$$
\epsilon=\frac{N_{X}^{\text {pass }}}{N_{X}^{\text {total }}}
$$

Since the probability for a given particle to pass the cuts is affected by the process from which the particle originates, and since different background processes have different kinematic distributions, the efficiencies are calculated separately for the various truth and reconstruction categories and binned in $p_{\mathrm{T}}$ and $|\eta|$. In $|\eta|$, the efficiencies are separated into two bins corresponding to the barrel $(|\eta|<1.37)$ and the end-cap $(1.37<|\eta|<2.47)$ since the efficiency distributions are approximately flat in each of these regions. Three $p_{\mathrm{T}}$ bins spanning the ranges $[7,10),[10,15)$, and $[15+] \mathrm{GeV}$ are chosen in order to have roughly equal statistical significance in each bin.

\subsubsection{Efficiencies for the reconstruction categories}

The average, unbinned efficiencies are listed in Table 6.5, while the binned efficiencies are plotted in Figure 6.1. As expected, the efficiencies are lower for fake-like objects, since the selection cuts are tuned to reject these objects. In general, the efficiencies are also roughly $15-20 \%$ lower for the Monte Carlo, due to mismodelling of the background processes. 
6. A data-driven estimation of the reducible electron-like background in the $H \rightarrow Z Z^{(*)} \rightarrow 4 \ell$ channel: the Transfer Factor method

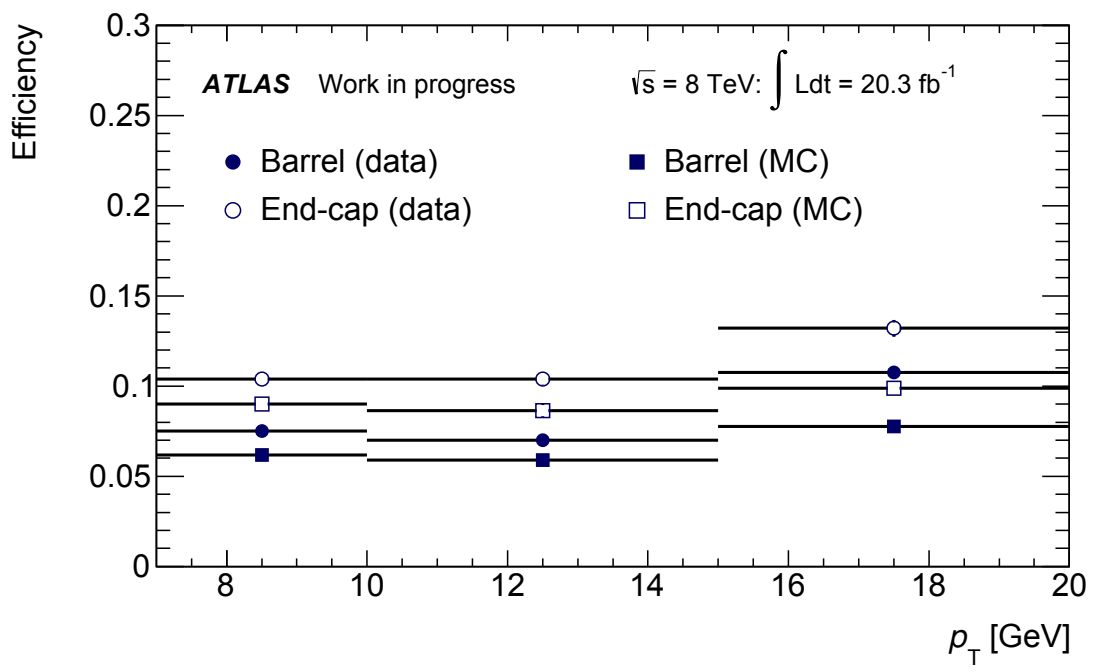

(a) Electron-like $(E)$

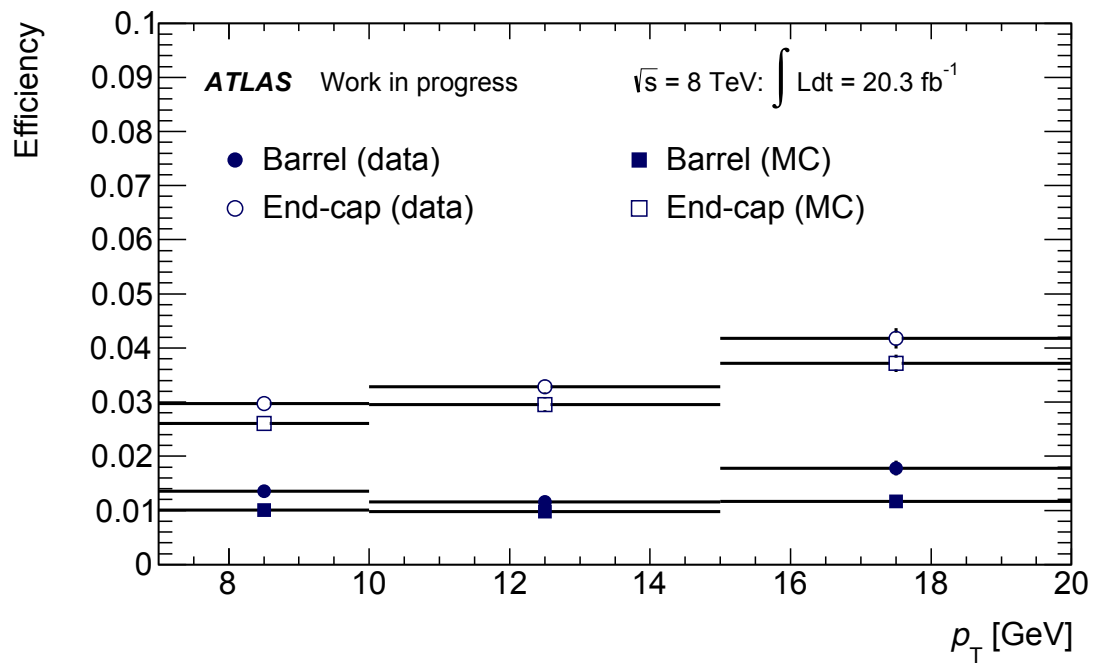

(b) Fake-like $(F)$

FIgURE 6.1: Reconstruction-categorized efficiencies for background objects passing the electron identification, isolation, and impact parameter significance cuts in the $8 \mathrm{TeV}$ data and Monte Carlo. Efficiencies are derived using the $Z+X$ control sample and calculated separately for electron-like and fake-like objects. (The statistical uncertainties are too small to be visible for most points.) 
6. A data-driven estimation of the reducible electron-like background in the $H \rightarrow Z Z^{(*)} \rightarrow 4 \ell$ channel: the Transfer Factor method

\begin{tabular}{ccc}
\hline \hline & \multicolumn{2}{c}{ Efficiency (\%) } \\
Category & Data & MC \\
\hline Global & $4.4 \pm 0.0$ & $3.6 \pm 0.0$ \\
$E$ & $9.0 \pm 0.1$ & $7.2 \pm 0.1$ \\
$F$ & $2.2 \pm 0.0$ & $1.9 \pm 0.0$ \\
\hline \hline
\end{tabular}

TABLE 6.5: Reconstruction-categorized efficiencies for uncategorized, electronlike $(E)$ and fake-like $(F)$ background objects passing the electron identification, isolation, and impact parameter significance cuts in the $8 \mathrm{TeV}$ data and Monte Carlo.

\begin{tabular}{ccc}
\hline \hline & \multicolumn{2}{c}{ Efficiency (\%) } \\
Category & $E$ & $F$ \\
\hline$e$ & $96.1 \pm 0.8$ & $85.9 \pm 3.4$ \\
$q$ & $17.3 \pm 0.4$ & $18.0 \pm 0.8$ \\
$\gamma$ & $8.9 \pm 0.2$ & $7.7 \pm 0.3$ \\
$f$ & $2.8 \pm 0.1$ & $1.2 \pm 0.0$ \\
\hline \hline
\end{tabular}

TABLE 6.6: Truth efficiencies for background objects passing the electron identification, isolation, and impact parameter significance cuts in the $8 \mathrm{TeV}$ Monte Carlo. Monte Carlo truth information is used to categorize objects as true isolated electrons $(e)$, electrons from heavy quark decays $(q)$, photon conversions and FSR $(\gamma)$, and fakes $(f)$. The efficiencies are calculated separately for the electron-like $(E)$ and fake-like $(F)$ reconstruction categories.

\subsubsection{Efficiencies for the truth categories}

The efficiencies for each of the four truth categories are also calculated using Monte Carlo. These are also separated into reconstruction categories; since some of the shower shape variables used in the electron identification menu (e.g. $f_{1}, R_{\phi}$ ) are also used to discriminate between reconstruction categories, it is expected that the truth efficiencies may vary depending on the reconstruction category of the truth object. The average efficiency values for each category are given in Table 6.6, while the binned values are plotted in Figure 6.2 - Figure 6.5. As one might expect, the 
6. A data-driven estimation of the reducible electron-like background in the $H \rightarrow Z Z^{(*)} \rightarrow 4 \ell$ channel: the Transfer Factor method

efficiencies for true isolated electrons in the electron-like reconstruction category are the highest at close to $100 \%$, and the lowest efficiencies are found for true fakes classified as being fake-like, while the efficiencies for non-prompt, non-isolated electrons fall in between the two extremes.

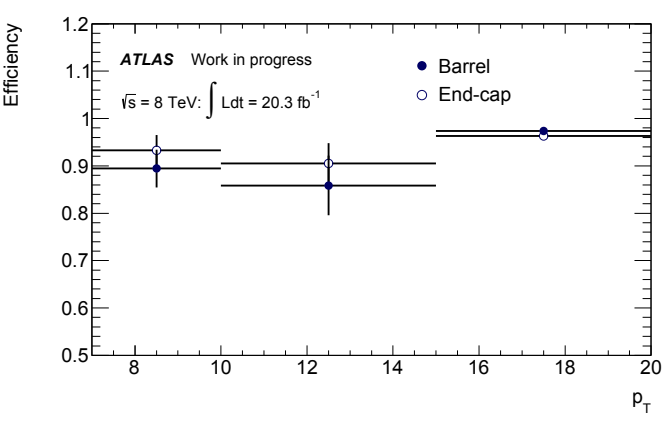

(a) Electron-like $(E)$

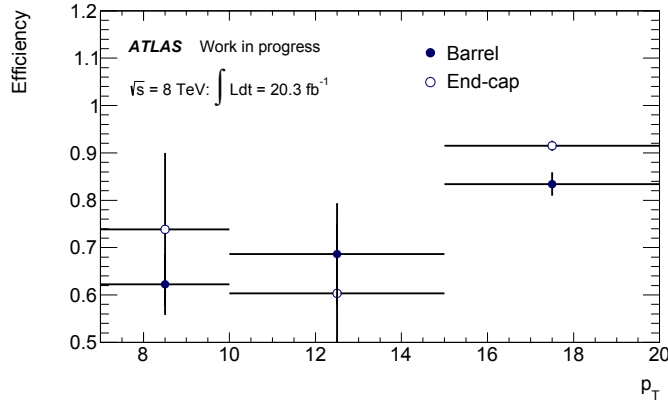

(b) Fake-like $(F)$

Figure 6.2: Truth efficiencies as a function of $p_{\mathrm{T}}$ for true isolated electrons $(e)$ passing the electron identification, isolation, and impact parameter significance cuts in the $8 \mathrm{TeV}$ Monte Carlo. The efficiencies are subdivided into electron-like and fake-like reconstruction categories and are shown separately for the barrel and end-cap regions.

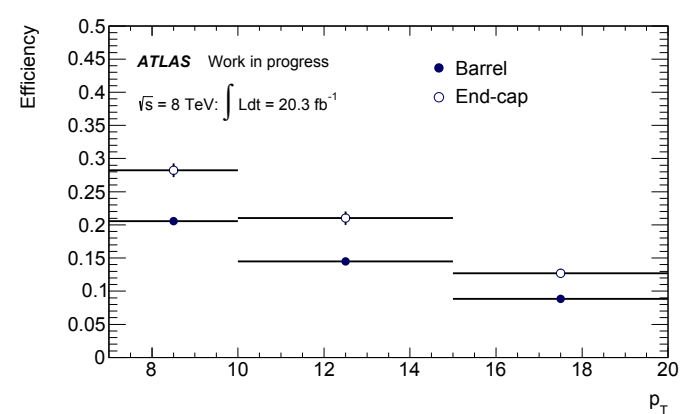

(a) Electron-like $(E)$

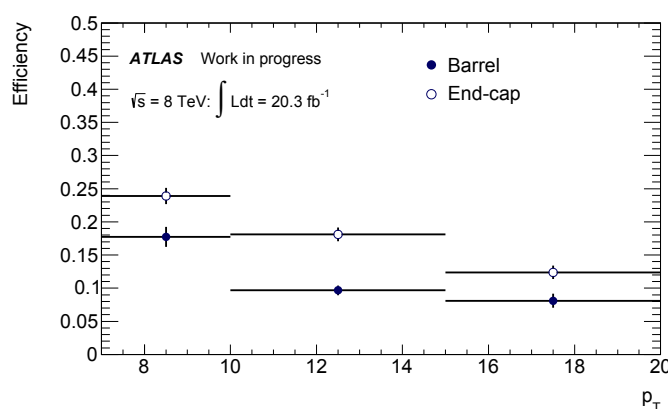

(b) Fake-like $(F)$

FiguRE 6.3: Truth efficiencies as a function of $p_{\mathrm{T}}$ for electrons from heavy quark decays $(q)$ passing the electron identification, isolation, and impact parameter significance cuts in the $8 \mathrm{TeV}$ Monte Carlo. The efficiencies are subdivided into electron-like and fake-like reconstruction categories and are shown separately for the barrel and end-cap regions. 


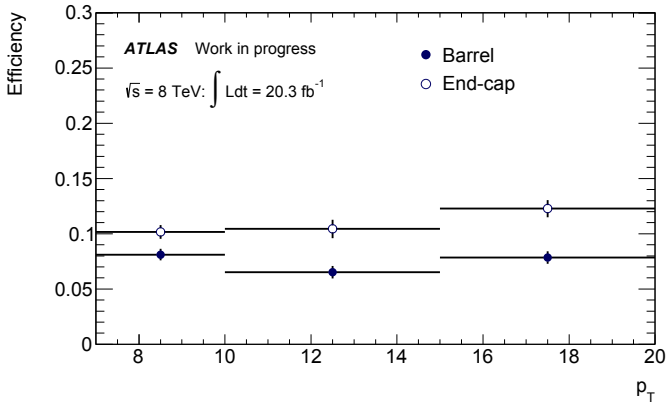

(a) Electron-like $(E)$

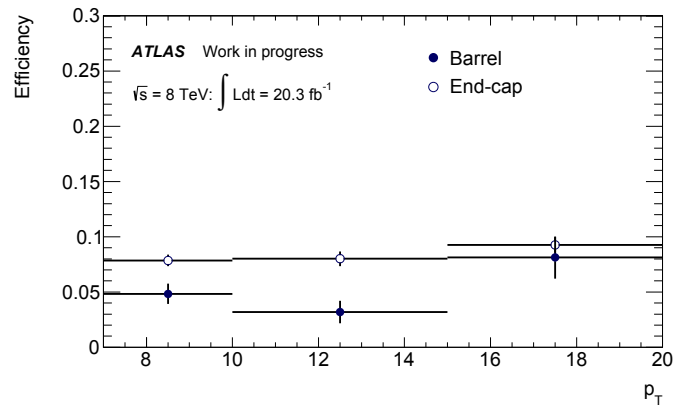

(b) Fake-like $(F)$

FiguRE 6.4: Truth efficiencies as a function of $p_{\mathrm{T}}$ for photon conversions and FSR $(\gamma)$ passing the electron identification, isolation, and impact parameter significance cuts in the $8 \mathrm{TeV}$ Monte Carlo. The efficiencies are subdivided into electron-like and fake-like reconstruction categories and are shown separately for the barrel and end-cap regions.

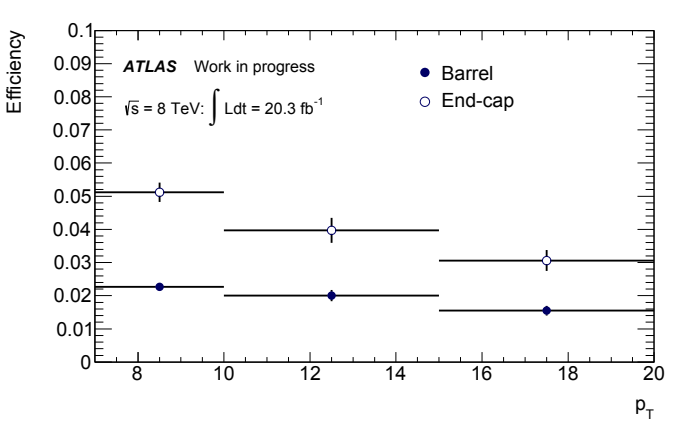

(a) Electron-like $(E)$

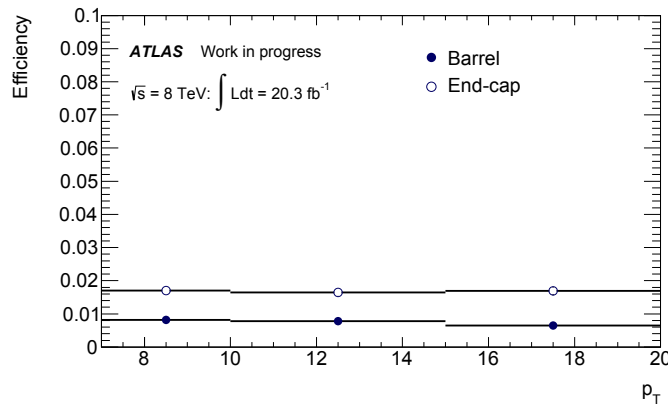

(b) Fake-like $(F)$

Figure 6.5: Truth efficiencies as a function of $p_{\mathrm{T}}$ for fakes $(f)$ passing the electron identification, isolation, and impact parameter significance cuts in the $8 \mathrm{TeV}$ Monte Carlo. The efficiencies are subdivided into electron-like and fakelike reconstruction categories and are shown separately for the barrel and endcap regions.

\subsubsection{Comparison of the $Z+X$ and $Z+X X$ samples and correction of the efficiencies}

It was shown in Section 6.3.2 and Section 6.3.3 that the efficiencies of the selection cuts vary depending on the true and reconstructed composition of the sample 
6. A data-driven estimation of the reducible electron-like background in the $H \rightarrow Z Z^{(*)} \rightarrow 4 \ell$ channel: the Transfer Factor method

from which they are derived. Hence, when using efficiencies to extrapolate the background yield from a control region, the composition of the control region and the sample used to calculate the efficiencies should match as closely as possible in order to ensure that the various background components are not over- or underrepresented in the final estimate. Since the $Z+X$ and $Z+X X$ samples correspond to different regions of phase space (events containing exactly three good reconstructed leptons versus events containing at least four good reconstructed leptons), there is no reason, a priori, why the two should have the same composition, so this must be checked and any discrepancies corrected for if necessary.

Table 6.7 shows the compositions of the $Z+X$ and $Z+X X$ samples in terms of the reconstruction and truth categories, determined using Monte Carlo. Each row corresponds to a particular reconstruction category and gives the percentage of $X$ in that category belonging to each of the four truth categories. In both samples, the proportion of true isolated electrons is less than $1 \%$, which indicates that the background control selection performs well in terms of rejecting signal and irreducible background events. In addition, over $90 \%$ of objects assigned to the fake-like reconstruction category are, in fact, true fakes in both cases.

\begin{tabular}{ccccc}
\hline \hline \multicolumn{5}{c}{$Z+X$ control sample } \\
Category & $e^{5}$ & $q$ & $\gamma$ & $f$ \\
\hline$E$ & $0.7 \pm 0.0$ & $17.6 \pm 0.1$ & $19.9 \pm 0.2$ & $61.8 \pm 0.3$ \\
$F$ & $0.1 \pm 0.0$ & $1.7 \pm 0.0$ & $5.4 \pm 0.1$ & $92.9 \pm 0.3$ \\
\hline \hline \multicolumn{5}{c}{$Z+X X$ control region } \\
Category & $e$ & $q$ & $\gamma$ & $f$ \\
\hline$E$ & $0.2 \pm 0.0$ & $34.8 \pm 1.1$ & $16.1 \pm 1.0$ & $48.9 \pm 1.9$ \\
$F$ & $0.0 \pm 0.0$ & $4.1 \pm 0.3$ & $5.3 \pm 0.4$ & $90.5 \pm 2.6$ \\
\hline \hline
\end{tabular}

TABLE 6.7: Composition of the $Z+X$ control sample used to determine the efficiencies, and of the $Z+X X$ control region using the $8 \mathrm{TeV}$ Monte Carlo. For each reconstruction category, $E$ and $F$, the percentage of $X$ belonging to each truth category is given. 
6. A data-driven estimation of the reducible electron-like background in the $H \rightarrow Z Z^{(*)} \rightarrow 4 \ell$ channel: the Transfer Factor method

However, Table 6.7 also shows some discrepancies in composition, particularly in the electron-like reconstruction category. In this category, the $Z+X X$ control region contains twice as many electrons from heavy quark decays as the $Z+X$ control sample, and roughly $20 \%$ fewer true fakes. Therefore, it was chosen to compensate for these differences by using the truth efficiencies, weighted by the composition of the $Z+X X$ control region and corrected for differences in the data and Monte Carlo efficiencies, to calculate the transfer factors. The general formula for the efficiencies is given in Equation 6.4.

$$
\epsilon_{E, F}=f_{E, F}^{e} \cdot \epsilon_{E, F}^{e}+f_{E, F}^{q} \cdot \epsilon_{E, F}^{q}+f_{E, F}^{\gamma} \cdot \epsilon_{E, F}^{\gamma} \cdot S F^{\gamma}+f_{E, F}^{f} \cdot \epsilon_{E, F}^{f} \cdot S F_{E, F}^{f} \cdot
$$

Here, the $f_{E, F}^{x}$ correspond to the fraction of objects in the $Z+X X$ control region assigned to the reconstruction category $\{E, F\}$ and belonging to the truth category $x=\{e, q, \gamma, f\}$, binned in $p_{\mathrm{T}}$ and $|\eta|$. The $\epsilon_{E, F}^{x}$ represent the efficiency for a given truth category $x$ assigned to the reconstruction category $\{E, F\}$, derived using the $Z+X$ Monte Carlo sample and binned in $p_{\mathrm{T}}$ and $|\eta| . S F^{\gamma}$ and $S F_{E, F}^{f}$ are scale factors derived to take into account differences in the data and Monte Carlo efficiencies; these are derived using enriched control regions, and will be described in more detail in Section 6.3.4.1. The efficiencies for $e$ and $q$ are not scaled; the electron efficiency scale factors described in Section 5.3.3.1 are applied to the former category during the event selection, while studies using $b$-jet enriched control samples have indicated that the efficiencies for electrons from heavy quark decays in data and Monte Carlo are compatible with one another [112]. 
6. A data-driven estimation of the reducible electron-like background in the $H \rightarrow Z Z^{(*)} \rightarrow 4 \ell$ channel: the Transfer Factor method

\subsubsection{Selection of enriched control regions and derivation of effi- ciency scale factors}

In order to correct for modelling differences in the Monte Carlo with respect to the data, special $Z+X$ control regions, enriched in photon conversions and fakes, were designed. The selection of these control regions is described below:

- $\gamma$-enriched control region This control region follows the standard $Z+X$ selection, but requires exactly zero B-layer hits; the B-layer hit requirement is also removed from the relaxed electron identification menu. In addition, a fraction of high-threshold TRT hits greater than $15 \%\left(R_{\mathrm{TRT}}>0.15\right)$ is required. This ensures that electrons originating from photon conversions are selected. The selection yields a sample of approximately 30,000 events, which is expected from Monte Carlo studies to be over $85 \%$ pure in electrons from photon conversions. Due to the low statistics, the $\gamma$-enriched control region is not separated into reconstruction categories or binned in $|\eta|$; the usual three $p_{\mathrm{T}}$ bins are used.

- Fake-enriched control region This control region follows the standard $Z+X$ selection, with the additional stipulation that at least one B-layer hit must occur. This requirement vetoes photon conversions and yields a sample containing approximately 250,000 events, $84 \%$ of which are true fakes. This control region is binned in $|\eta|$ and $p_{\mathrm{T}}$ and separated into reconstruction categories as usual. The $F$ sample is over $90 \%$ pure, while the $E$ sample is approximately $70 \%$ pure, according to Monte Carlo studies.

Once the enriched control regions have been constructed using both data and Monte Carlo, the efficiencies for photon conversions and fakes are determined 
6. A data-driven estimation of the reducible electron-like background in the $H \rightarrow Z Z^{(*)} \rightarrow 4 \ell$ channel: the Transfer Factor method

as follows. The procedure is straightforward for the Monte Carlo, due to the available truth information; the efficiency is simply the ratio of the number of true $\gamma$ or $f$ passing the identification, isolation, and impact parameter significance cuts $\left(N_{\mathrm{MC}}^{\gamma, f}\right.$ pass $)$ to the total number of those objects in the enriched control region $\left(N_{\mathrm{MC}}^{\gamma, f}\right)$ :

$$
\epsilon_{\mathrm{MC}}^{\gamma, f}=\frac{N_{\mathrm{MC}}^{\gamma, f \text { pass }}}{N_{\mathrm{MC}}^{\gamma, f}} .
$$

For the data, since the objects' true identities are unknown, the numerator and denominator of Equation 6.5 are determined by taking the number of events in the data control region enriched in a particular truth category and subtracting the contributions of the components belonging to the other truth categories as determined using the Monte Carlo control region:

$$
\epsilon_{\mathrm{Data}}^{\gamma, f}=\frac{N_{\text {Data }}^{\gamma, f \text { pass }}-N_{\mathrm{MC}}^{f, \gamma \text { pass }}-N_{\mathrm{MC}}^{e}{ }^{e \text { pass }}-N_{\mathrm{MC}}^{q \text { pass }}}{N_{\text {Data }}^{\gamma, f}-N_{\mathrm{MC}}^{f, \gamma}-N_{\mathrm{MC}}^{e}-N_{\mathrm{MC}}^{q}} .
$$

Then, the efficiency scale factors are determined by computing the ratio of the efficiency calculated using data to that found using Monte Carlo:

$$
S F^{\gamma, f}=\frac{\epsilon_{\mathrm{Data}}^{\gamma, f}}{\epsilon_{\mathrm{MC}}^{\gamma, f}}
$$

The efficiency scale factors are calculated simultaneously, using an iterative process: the scale factor found for one component is used to correct the truth composition of that component in the other control region, and vice versa, until the scale factors reach completely stable values. In practice, this requires only a few iterations; typically on the order of two or three. The resulting scale factors, which are used to correct the Monte Carlo truth efficiencies in Equation 6.4, are listed in Table 6.8. 
6. A data-driven estimation of the reducible electron-like background in the $H \rightarrow Z Z^{(*)} \rightarrow 4 \ell$ channel: the Transfer Factor method

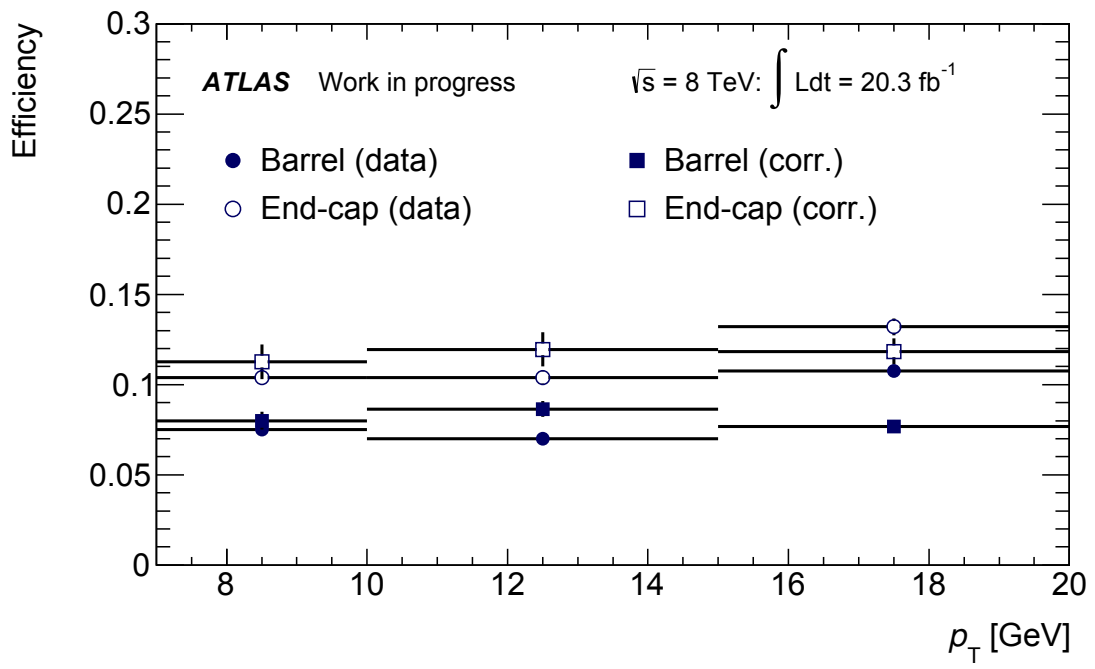

(a) Electron-like $(E)$

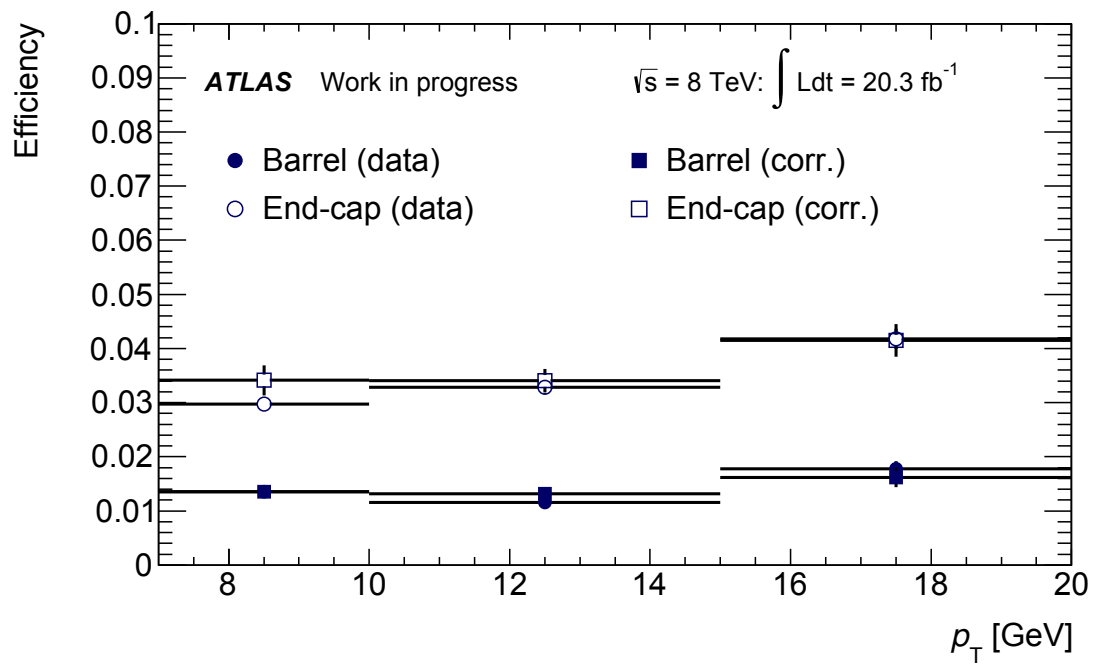

(b) Fake-like $(F)$

Figure 6.6: Truth efficiencies as a function of $p_{\mathrm{T}}$, taken from Monte Carlo and corrected to match the expected $Z+X X$ composition and to take into account differences in the data and Monte Carlo efficiencies. The original, uncorrected efficiencies taken from the data are shown for comparison. 
6. A data-driven estimation of the reducible electron-like background in the $H \rightarrow Z Z^{(*)} \rightarrow 4 \ell$ channel: the Transfer Factor method

\begin{tabular}{cccc}
\hline \hline \multicolumn{4}{c}{$p_{\mathrm{T}}[\mathrm{GeV}]$} \\
& $7-10$ & $10-15$ & $15+$ \\
\hline \multicolumn{4}{c}{$S F^{\gamma}$} \\
\hline Global & $1.09 \pm 0.03$ & $1.00 \pm 0.03$ & $1.19 \pm 0.04$ \\
\hline \multicolumn{4}{c}{$S F^{f}$} \\
$F$ & $1.30 \pm 0.04$ & $1.93 \pm 0.11$ & $3.13 \pm 0.23$ \\
$F$ & $1.22 \pm 0.03$ & $1.27 \pm 0.06$ & $1.16 \pm 0.08$ \\
\hline \hline
\end{tabular}

TABLE 6.8: Scale factors used to correct Monte Carlo truth efficiencies for the photon conversion $(\gamma)$ and fake $(f)$ components in the $8 \mathrm{TeV}$ reducible background analysis. The scale factors for the $f$ component are separated into electron-like $(E)$ and fake-like $(F)$ reconstruction categories. The statistical uncertainties are also given.

The final truth $Z+X$ efficiencies, corrected to match the expected $Z+X X$ composition and to take into account differences in the data and Monte Carlo efficiencies, are shown in Figure 6.6, along with the original uncorrected efficiencies from data for comparison. The effect of the correction is minimal for the fake-like reconstruction category, where the compositions of the $Z+X$ and $Z+X X$ control regions are in good agreement, but a more significant difference can be seen for the electron-like category, where the compositions of the two control regions are not as well-matched.

\subsection{Systematic uncertainties}

The systematic uncertainties on the reducible background yields are derived from three main sources.

- Statistical uncertainty of the efficiency scale factors A conservative estimate is made by taking this uncertainty from the $p_{\mathrm{T}}$ bin with the largest statistical uncertainty, i.e. the $p_{\mathrm{T}}>15 \mathrm{GeV}$ bin (refer to Table 6.8). 
6. A data-driven estimation of the reducible electron-like background in the $H \rightarrow Z Z^{(*)} \rightarrow 4 \ell$ channel: the Transfer Factor method

Weighted to take into account the proportion of electron-like and fake-like background objects in the $Z+X X$ control region, this amounts to a contribution of $5 \%$ of the yield due to the uncertainty of the $f$ efficiency scale factor and $2 \%$ due to the $\gamma$ efficiency scale factor.

- Statistical uncertainty of the $Z+X X$ composition To determine the effect of statistical fluctuations in the composition of the control region, the reducible background estimate was recalculated using the unbinned values of the efficiencies and composition. Compared to the nominal estimate, this was found to have an effect of 0.43 events $(18 \%)$ in the $4 e$ channel and 0.34 events $(13 \%)$ in the $2 \mu 2 e$ channel.

- Residual mismodelling differences in the efficiencies The efficiency scale factors were adjusted by an amount corresponding to their bin-by-bin variation (refer to Table 6.8): $10 \%$ for $S F_{F}^{f}, 35 \%$ for $S F_{E}^{f}$, and $20 \%$ for $S F^{\gamma}$. The efficiency for electrons from heavy quark decays, which was found to agree within an uncertainty of $10 \%$ for data and Monte Carlo, was varied by this amount. This led to a variation in the final reducible background estimate of 0.89 events $(36 \%)$ in the $4 e$ channel and 0.90 events $(36 \%)$ in the $2 \mu 2 e$ channel.

\subsection{Results of the Transfer Factor method using the $8 \mathrm{TeV}$ data}

For each event in the $Z+X X$ control region selected from the $8 \mathrm{TeV}$ data as described in Section 6.2, the reconstruction category-, $p_{\mathrm{T}^{-}}$, and $|\eta|$-dependent transfer factor is calculated using Equation 6.2 and the corrected efficiencies are determined 
6. A data-driven estimation of the reducible electron-like background in the $H \rightarrow Z Z^{(*)} \rightarrow 4 \ell$ channel: the Transfer Factor method

using Equation 6.4. The transfer factors are summed over all of the events in the control region to obtain a preliminary estimate of the reducible background yield in the signal region.

It was found that the method, as applied in this control region, tends to suppress the contribution of the $W Z$ process, and so the expected number of $W Z$ events surviving the full analysis selection is determined using Monte Carlo and added to the preliminary estimate. For completeness, the Transfer Factor method is also applied to the simulated $Z Z^{(*)}$ background sample, and the resulting negligible contribution is subtracted. The results are presented in Table 6.9. Compared to the results of the baseline $3 \ell+X$ method described in Section 5.4.2.2, the Transfer Method produces compatible results, within the uncertainties.

\begin{tabular}{ccc}
\hline \hline & $4 e$ & $2 \mu 2 e$ \\
\hline Data & $1.90 \pm 0.07$ (stat.) & $2.09 \pm 0.08$ (stat.) \\
$Z Z^{(*)}(\mathrm{MC})$ & $0.01 \pm 0.00$ (stat.) & $0.01 \pm 0.00$ (stat.) \\
$W Z(\mathrm{MC})$ & $0.56 \pm 0.06$ (stat.) & $0.44 \pm 0.07$ (stat.) \\
Final estimate & $2.45 \pm 0.10$ (stat.) \pm 0.89 (syst.) & $2.52 \pm 0.10$ (stat.) \pm 0.90 (syst.) \\
\hline Baseline estimate & $2.88 \pm 0.28$ (stat.) \pm 0.54 (syst.) & $2.91 \pm 0.33$ (stat.) \pm 0.60 (syst.) \\
\hline \hline
\end{tabular}

TABLE 6.9: The reducible background estimate for the $8 \mathrm{TeV}$ data, determined using the Transfer Factor method. The preliminary estimate is obtained from data, and the $Z Z^{(*)}$ contribution obtained using the method on Monte Carlo is subtracted. An additional contribution from the $W Z$ process is added using the full analysis selection on Monte Carlo to obtain the final reducible background yield in the signal region. For comparison purposes, the baseline estimate obtained using the $3 \ell+X$ method as described in Section 5.4.2.2 is also provided. 


\subsection{Modification of the Transfer Factor method for the $7 \mathrm{TeV}$ analysis}

The Transfer Factor method was originally developed for the analysis of the $8 \mathrm{TeV}$ data. Once it was validated against the existing baseline method, the method was then applied to the $7 \mathrm{TeV}$ data. The reduced statistics and different analysis procedure (particularly the different electron identification menu) used for this analysis, as described in Chapter 5, necessitated several modifications to the Transfer Factor method; these will be discussed below.

\subsection{1 $Z+X X$ control regions in the $7 \mathrm{TeV}$ data}

For the purpose of the reducible background estimation, the main difference in the selection analyses for the $7 \mathrm{TeV}$ and $8 \mathrm{TeV}$ data comes from the electron identification menus. For the $7 \mathrm{TeV}$ analysis, the Loose ++ menu defined in Section 4.2 was used; this menu accepts approximately 2.5 times more background than the Loose LH menu. In addition, it does not feature a B-layer hit requirement, which means that the fraction of events originating from photon conversions will be higher in the $7 \mathrm{TeV}$ data.

A major challenge for a data-driven background estimate using the $7 \mathrm{TeV}$ data comes from the lower integrated luminosity: $4.5 \mathrm{fb}^{-1}$ recorded at $7 \mathrm{TeV}$ in 2011, versus $20.3 \mathrm{fb}^{-1}$ at $8 \mathrm{TeV}$ in 2012 . Even with the looser background rejection from the electron identification menu, the control regions will be statistically limited. 
6. A data-driven estimation of the reducible electron-like background in the $H \rightarrow Z Z^{(*)} \rightarrow 4 \ell$ channel: the Transfer Factor method

Two control regions were investigated for the $7 \mathrm{TeV}$ analysis due to the lack of statistics; the first being the $Z+X X$ control region described in Section 6.2. For historical reasons, this control region is denoted by CR5a, and this name will be used to distinguish it from this point. The second control region has slightly looser requirements and thus allows for a larger sample from which to estimate the background yield; only one of the subleading $X$ is required to fail the identification, isolation, or impact parameter significance cuts. This control region is denoted by CR5. The transfer factor for this control region is given by:

$$
T F_{5}=\frac{\epsilon_{3} \cdot \epsilon_{4}}{1-\epsilon_{3} \cdot \epsilon_{4}}
$$

Note that CR5a is a subset of CR5. Table 6.10 and Table 6.11 give the number of events in the two control regions in the $7 \mathrm{TeV}$ data, as well as the expected number of events from Monte Carlo. It is apparent that CR5a is severely statistically limited, and so it has been decided that the primary estimate for this method should be taken from CR5. For the sake of completeness, the background yield will also be estimated using CR5a. Table 6.12 and Table 6.13 give the composition of the control regions in terms of the reconstruction categories defined in Section 6.1.2.

\begin{tabular}{ccc}
\hline \hline & $4 e$ & $2 \mu 2 e$ \\
\hline Data & $62.0 \pm 7.9$ & $67.0 \pm 8.2$ \\
\hline$Z+$ jets & $39.3 \pm 3.3$ & $47.0 \pm 3.1$ \\
$Z+b \bar{b}$ & $3.3 \pm 0.2$ & $5.6 \pm 0.3$ \\
$t \bar{t}$ & $1.4 \pm 0.2$ & $1.9 \pm 0.2$ \\
$W Z$ & $0.0 \pm 0.0$ & $0.0 \pm 0.0$ \\
Total & $44.0 \pm 3.3$ & $54.5 \pm 3.1$ \\
\hline \hline
\end{tabular}

TABLE 6.10: Observed and expected yields in the $Z+X X$ control region CR5 in the $7 \mathrm{TeV}$ data and Monte Carlo. 
6. A data-driven estimation of the reducible electron-like background in the $H \rightarrow Z Z^{(*)} \rightarrow 4 \ell$ channel: the Transfer Factor method

\begin{tabular}{ccc}
\hline \hline & $4 e$ & $2 \mu 2 e$ \\
\hline Data & $38.0 \pm 6.2$ & $40.0 \pm 6.3$ \\
\hline$Z+$ jets & $23.3 \pm 2.2$ & $28.9 \pm 2.2$ \\
$Z+b \bar{b}$ & $2.0 \pm 0.2$ & $3.4 \pm 0.3$ \\
$t \bar{t}$ & $1.0 \pm 0.2$ & $1.3 \pm 0.2$ \\
$W Z$ & $0.0 \pm 0.0$ & $0.0 \pm 0.0$ \\
Total & $26.3 \pm 2.2$ & $32.7 \pm 2.2$ \\
\hline \hline
\end{tabular}

TABLE 6.11: Observed and expected yields in the $Z+X X$ control region CR5a in the $7 \mathrm{TeV}$ data and Monte Carlo.

\begin{tabular}{ccc}
\hline \hline Category & $4 e$ & $2 \mu 2 e$ \\
\hline$E E$ & $3.0 \pm 1.7$ & $2.0 \pm 1.4$ \\
$E F$ & $10.0 \pm 3.1$ & $9.0 \pm 3.0$ \\
$F E$ & $13.0 \pm 3.6$ & $15.0 \pm 3.9$ \\
$F F$ & $36.0 \pm 6.0$ & $41.0 \pm 6.4$ \\
\hline \hline
\end{tabular}

TABle 6.12: Composition of the $Z+X X$ control region CR5 in terms of the number of events observed in each of the reconstruction categories, as observed in the $7 \mathrm{TeV}$ data.

\begin{tabular}{ccc}
\hline \hline Category & $4 e$ & $2 \mu 2 e$ \\
\hline$E E$ & $1.0 \pm 1.0$ & $1.0 \pm 1.0$ \\
$E F$ & $6.0 \pm 2.4$ & $5.0 \pm 2.2$ \\
$F E$ & $5.0 \pm 2.2$ & $8.0 \pm 2.8$ \\
$F F$ & $26.0 \pm 5.1$ & $26.0 \pm 5.1$ \\
\hline \hline
\end{tabular}

TABLE 6.13: Composition of the $Z+X X$ control region CR5a in terms of the number of events observed in each of the reconstruction categories, as observed in the $7 \mathrm{TeV}$ data. 


\subsection{2 $Z+X$ efficiencies in the $7 \mathrm{TeV}$ data}

\begin{tabular}{ccc}
\hline \hline & \multicolumn{2}{c}{ Efficiency $(\%)$} \\
Category & Data & MC \\
\hline Global & $18.1 \pm 0.2$ & $17.5 \pm 0.2$ \\
$E$ & $25.1 \pm 0.6$ & $22.2 \pm 0.6$ \\
$F$ & $16.6 \pm 0.2$ & $16.6 \pm 0.2$ \\
\hline \hline
\end{tabular}

TABLE 6.14: Reconstruction-categorized efficiencies for uncategorized, electron-like $(E)$ and fake-like $(F)$ background objects passing the electron identification, isolation, and impact parameter significance cuts in the $7 \mathrm{TeV}$ data and Monte Carlo.

\begin{tabular}{ccc}
\hline \hline & \multicolumn{2}{c}{ Efficiency (\%) } \\
Category & $E$ & $F$ \\
\hline$e$ & $94.0 \pm 1.9$ & $91.3 \pm 4.7$ \\
$q$ & $32.5 \pm 1.6$ & $36.4 \pm 3.2$ \\
$\gamma$ & $25.3 \pm 1.3$ & $34.3 \pm 0.7$ \\
$f$ & $13.0 \pm 0.7$ & $11.7 \pm 0.2$ \\
\hline \hline
\end{tabular}

TABLE 6.15: Truth efficiencies for background objects passing the electron identification, isolation, and impact parameter significance cuts in the $7 \mathrm{TeV}$ Monte Carlo. Monte Carlo truth information is used to categorize objects as true isolated electrons $(e)$, electrons from heavy quark decays $(q)$, photon conversions and FSR $(\gamma)$, and fakes $(f)$. The efficiencies are calculated separately for the electron-like $(E)$ and fake-like $(F)$ reconstruction categories.

The efficiencies for $Z+X$ events to pass the electron identification, isolation, and impact parameter significance cuts are determined using the method described in Section 6.3.1, with the Loose++ identification used instead of the Loose LH operating point. The average efficiencies are shown in Table 6.14 for the reconstruction categories, and in Table 6.15 for the truth categories. As expected, the selection efficiencies for electron-like background objects are much higher due to the Loose++ electron identification menu (cf. Table 6.5 and Table 6.6). The 
6. A data-driven estimation of the reducible electron-like background in the $H \rightarrow Z Z^{(*)} \rightarrow 4 \ell$ channel: the Transfer Factor method

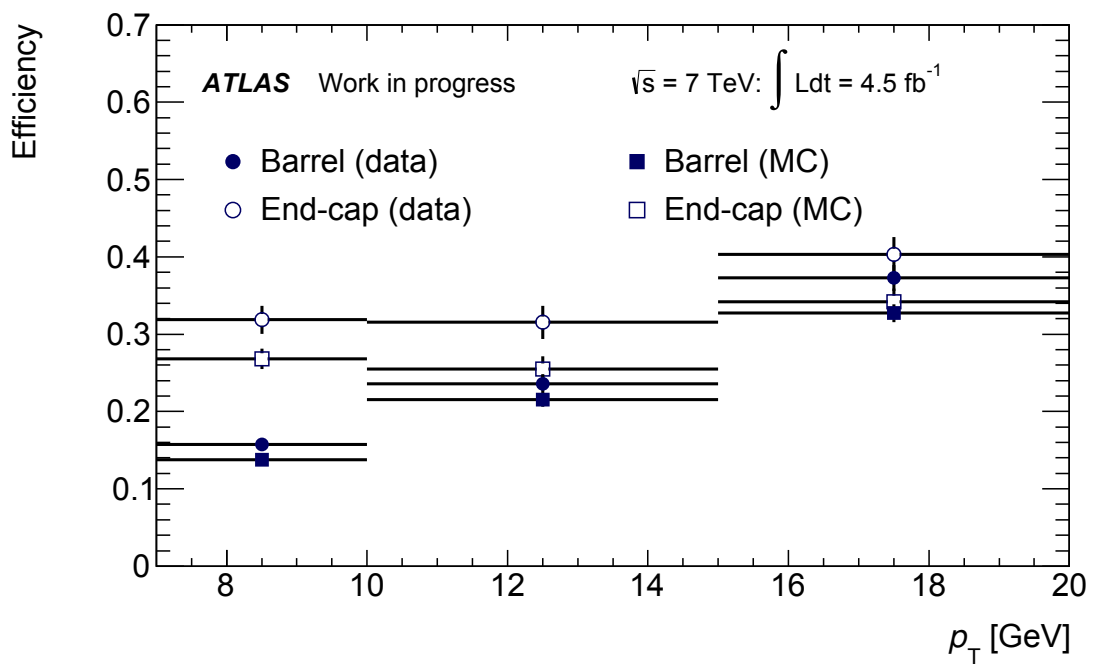

(a) Electron-like $(E)$

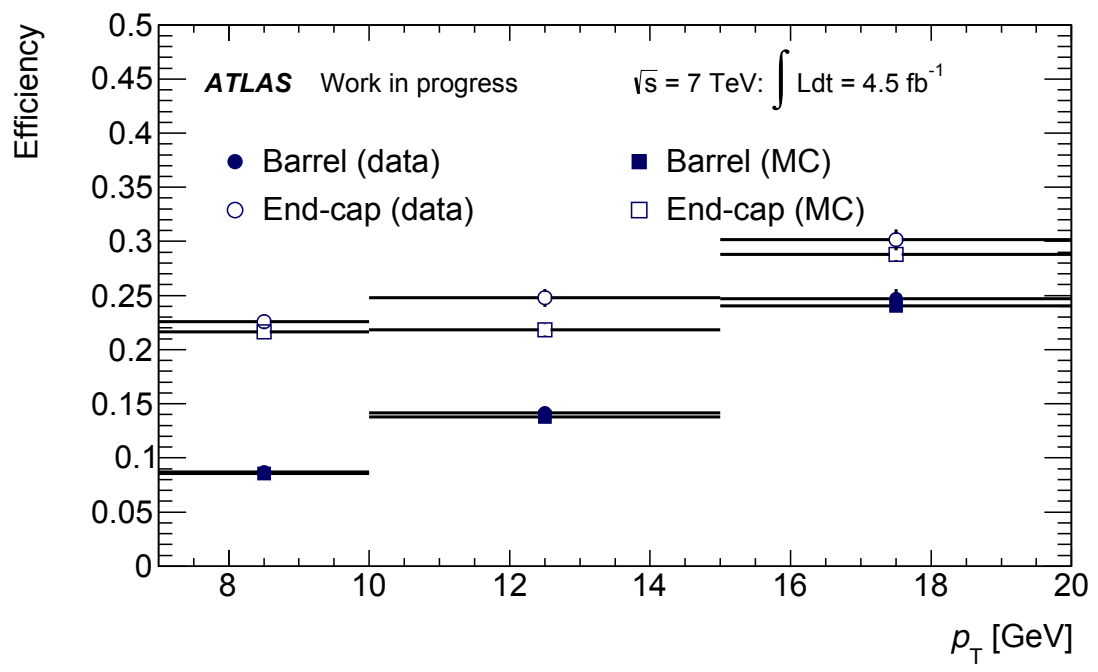

(b) Fake-like $(F)$

FiguRE 6.7: Reconstruction-categorized efficiencies for background objects passing the electron identification, isolation, and impact parameter significance cuts in the $7 \mathrm{TeV}$ data and Monte Carlo. Efficiencies are derived using the $Z+X$ control sample and calculated separately for electron-like and fake-like objects.

binned efficiencies for the reconstruction categories are shown in Figure 6.7, while the binned truth efficiencies are shown in Figure 6.8 - Figure 6.11. 


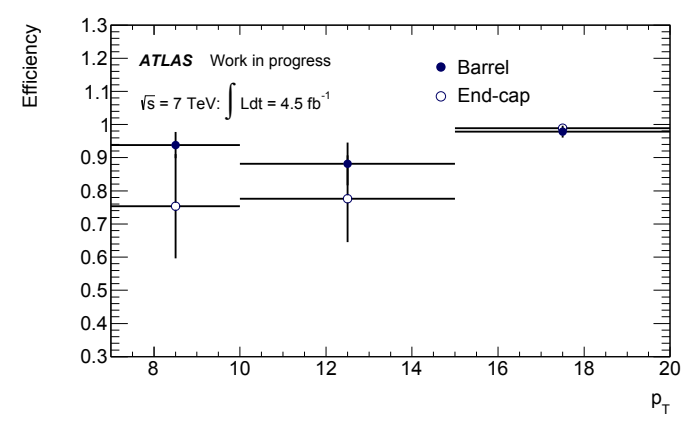

(a) Electron-like $(E)$

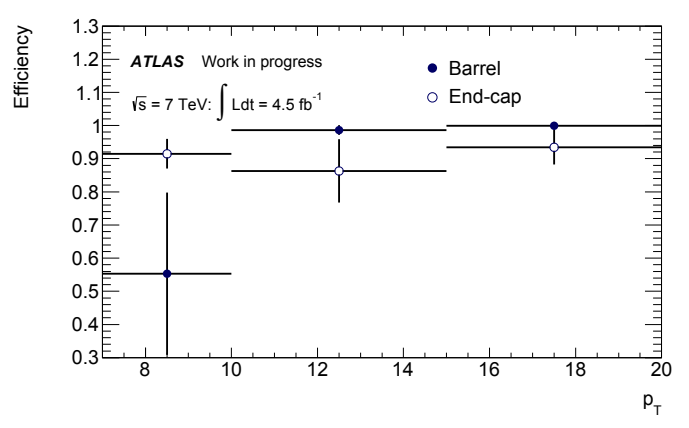

(b) Fake-like $(F)$

FiguRE 6.8: Truth efficiencies as a function of $p_{\mathrm{T}}$ for true isolated electrons $(e)$ passing the electron identification, isolation, and impact parameter significance cuts in the $7 \mathrm{TeV}$ Monte Carlo. The efficiencies are subdivided into electron-like and fake-like reconstruction categories and are shown separately for the barrel and end-cap regions.

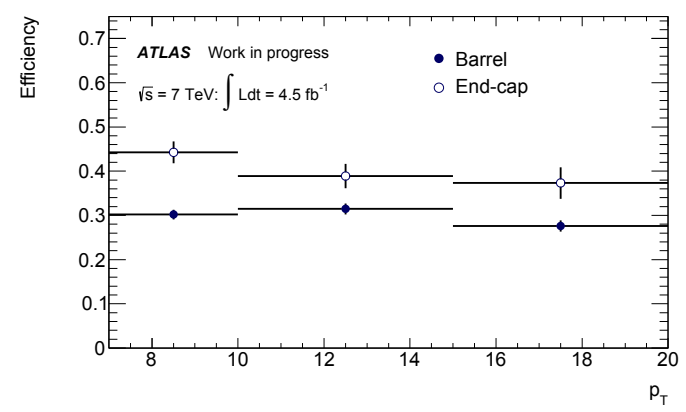

(a) Electron-like $(E)$

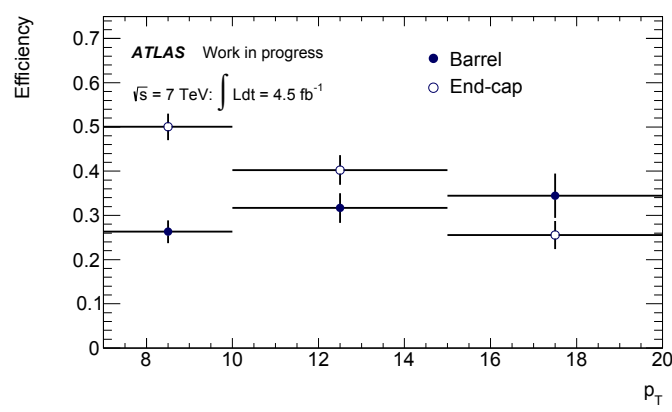

(b) Fake-like $(F)$

FiguRE 6.9: Truth efficiencies as a function of $p_{\mathrm{T}}$ for electrons from heavy quark decays $(q)$ passing the electron identification, isolation, and impact parameter significance cuts in the $7 \mathrm{TeV}$ Monte Carlo. The efficiencies are subdivided into electron-like and fake-like reconstruction categories and are shown separately for the barrel and end-cap regions. 


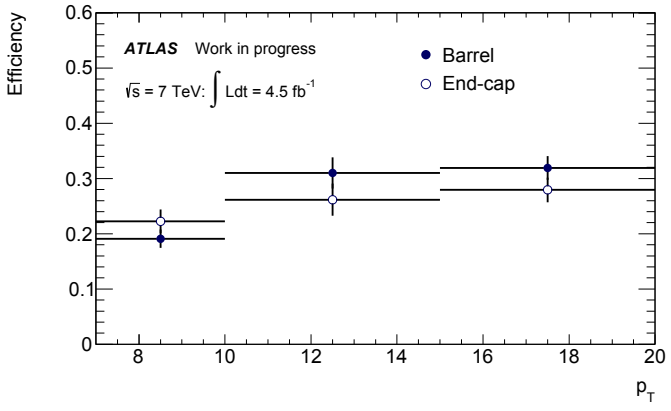

(a) Electron-like $(E)$

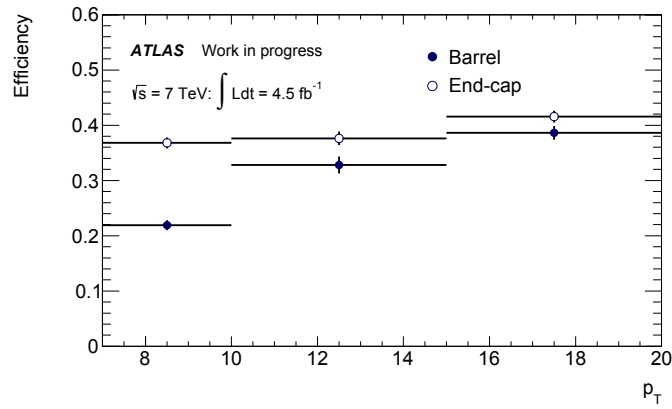

(b) Fake-like $(F)$

FiguRE 6.10: Truth efficiencies as a function of $p_{\mathrm{T}}$ for photon conversions and FSR $(\gamma)$ passing the electron identification, isolation, and impact parameter significance cuts in the $7 \mathrm{TeV}$ Monte Carlo. The efficiencies are subdivided into electron-like and fake-like reconstruction categories and are shown separately for the barrel and end-cap regions.

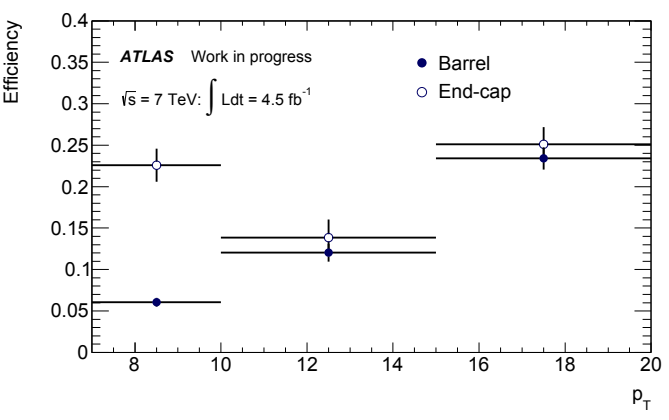

(a) Electron-like $(E)$

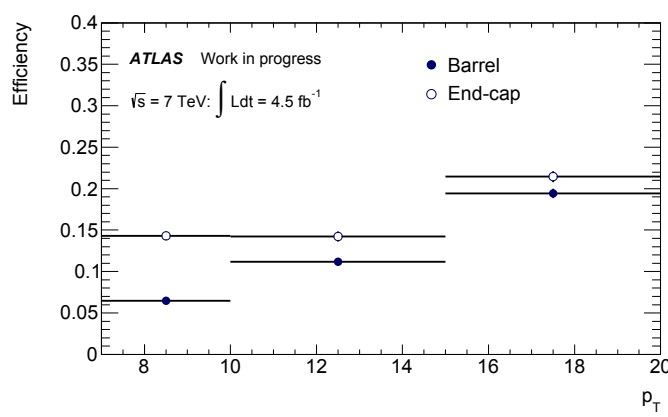

(b) Fake-like $(F)$

Figure 6.11: Truth efficiencies as a function of $p_{\mathrm{T}}$ for fakes $(f)$ passing the electron identification, isolation, and impact parameter significance cuts in the $7 \mathrm{TeV}$ Monte Carlo. The efficiencies are subdivided into electron-like and fakelike reconstruction categories and are shown separately for the barrel and endcap regions.

The compositions of the $Z+X$ and $Z+X X$ control regions are given in Table 6.16. Notable features include the higher fraction of true $\gamma$ objects due to the absence of the B-layer hit requirement in the Loose ++ menu, and the higher fraction of true $e$ objects in CR5. This latter observation is due to the fact that 
6. A data-driven estimation of the reducible electron-like background in the $H \rightarrow Z Z^{(*)} \rightarrow 4 \ell$ channel: the Transfer Factor method

CR5 requires that only one $X$ fail a selection cut; this allows a significant contribution from $Z Z^{(*)}$, which will be subtracted when the final reducible background estimate is determined.

\begin{tabular}{ccccc}
\hline \hline \multicolumn{5}{c}{$Z+X$ control sample } \\
Category & $e^{5}$ & $q$ & $\gamma$ & $f$ \\
\hline$E$ & $3.4 \pm 0.4$ & $18.3 \pm 0.3$ & $23.6 \pm 0.5$ & $54.7 \pm 0.9$ \\
$F$ & $0.1 \pm 0.0$ & $0.9 \pm 0.0$ & $20.1 \pm 0.2$ & $78.8 \pm 0.5$ \\
\hline \hline \multicolumn{5}{c}{$Z+X X$ control region (CR5) } \\
Category & $e$ & $q$ & $\gamma$ & $f$ \\
\hline$E$ & $10.9 \pm 3.6$ & $28.5 \pm 2.4$ & $24.7 \pm 3.7$ & $35.9 \pm 3.9$ \\
$F$ & $0.6 \pm 0.3$ & $1.3 \pm 0.1$ & $20.1 \pm 2.0$ & $77.9 \pm 4.4$ \\
\hline \hline & $Z+X X$ & control region (CR5a) \\
Category & $e$ & $q$ & $\gamma$ & $f$ \\
\hline$E$ & $0.1 \pm 0.1$ & $31.1 \pm 3.2$ & $21.4 \pm 3.6$ & $47.4 \pm 5.9$ \\
$F$ & $0.1 \pm 0.1$ & $1.3 \pm 0.2$ & $14.9 \pm 1.9$ & $83.7 \pm 5.3$ \\
\hline \hline
\end{tabular}

TABLE 6.16: Composition (in \%) of the $Z+X$ control sample used to determine the efficiencies, and of the $Z+X X$ control region using the $7 \mathrm{TeV}$ Monte Carlo. For each reconstruction category, $E$ and $F$, the percentage of $X$ belonging to each truth category is given.

The procedure for correcting the Monte Carlo truth efficiencies to match the $Z+X X$ composition varies slightly for the $7 \mathrm{TeV}$ data. Since, as Table 6.14 shows, the agreement in the efficiencies between data and Monte Carlo is quite fair, the efficiencies are not scaled. This is equivalent to setting the scale factors $S F^{\gamma}=1$ and $S F_{E, F}^{f}=1$ in Equation 6.4 when correcting the efficiencies. The corrected efficiencies are shown in Figure 6.12, with the uncorrected efficiencies from data for comparison. 
6. A data-driven estimation of the reducible electron-like background in the $H \rightarrow Z Z^{(*)} \rightarrow 4 \ell$ channel: the Transfer Factor method

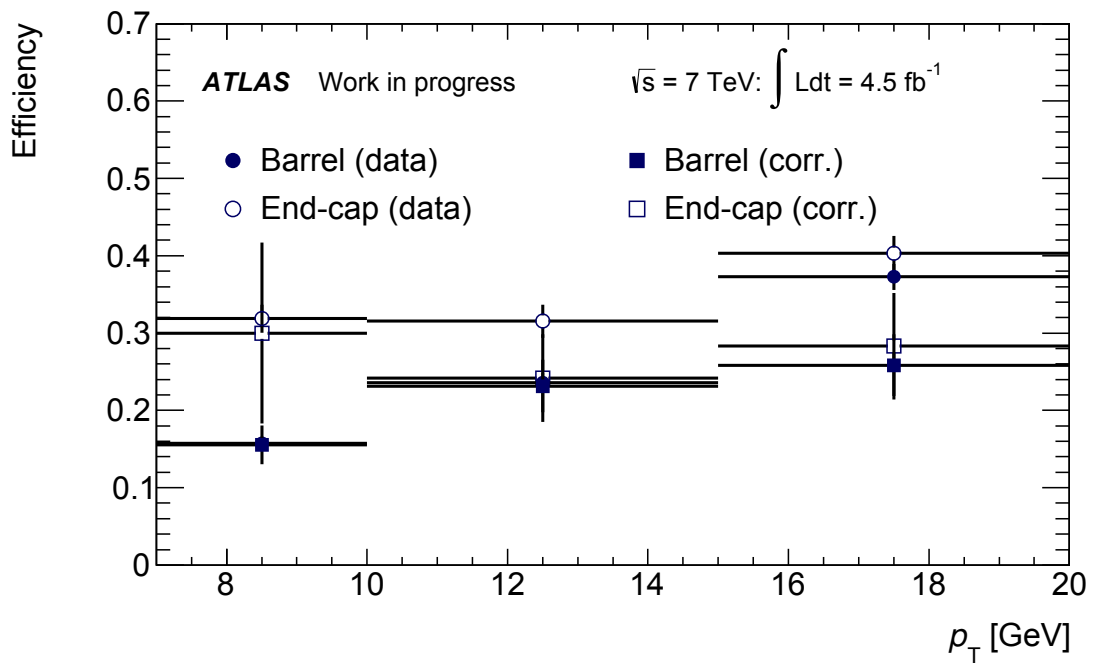

(a) Electron-like $(E)$

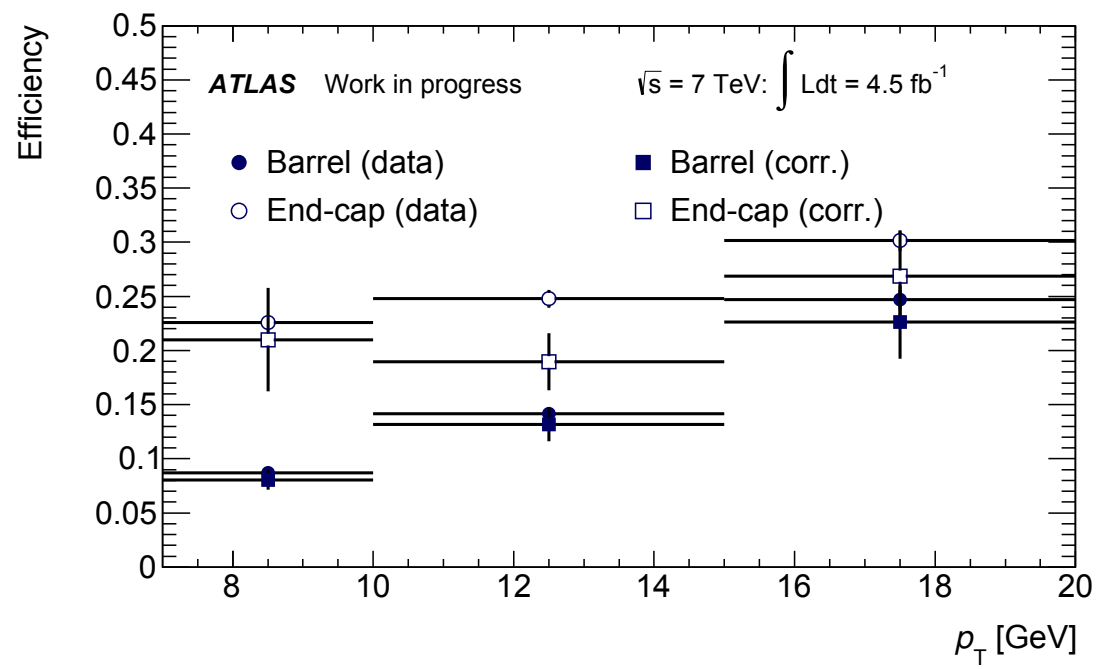

(b) Fake-like $(F)$

Figure 6.12: Truth efficiencies for the $7 \mathrm{TeV}$ analysis taken from Monte Carlo, corrected to match the expected $Z+X X$ composition and to take into account differences in the data and Monte Carlo efficiencies. The original, uncorrected efficiencies taken from the data are shown for comparison. 
6. A data-driven estimation of the reducible electron-like background in the $H \rightarrow Z Z^{(*)} \rightarrow 4 \ell$ channel: the Transfer Factor method

\subsubsection{Systematic uncertainties}

The systematic uncertainties are evaluated in a manner similar to that used for the $8 \mathrm{TeV}$ analysis, taking the following sources into consideration:

- Residual mismodelling differences in the efficiencies Since the efficiencies derived from data and Monte Carlo were found to be in good agreement, no scale factors were applied. To check the effect of variations in the efficiencies, the truth efficiencies were varied by $20 \%$. In CR5, this led to a variation in the final reducible background estimate of 0.91 events (35\%) in the $4 e$ channel and 1.03 events $(37 \%)$ in the $2 \mu 2 e$ channel. In CR5a, this led to a variation of 0.90 events $(44 \%)$ in the $4 e$ channel and 1.00 events (45\%) in the $2 \mu 2 e$ channel.

- Statistical uncertainty of the $Z+X X$ composition To determine the effect of statistical fluctuations in the composition of the control region, the reducible background estimate was recalculated using the unbinned values of the efficiencies and composition. In CR5, this was found to cause a variation of 0.05 events $(2 \%)$ in the $4 e$ channel and 0.16 events $(6 \%)$ in the $2 \mu 2 e$ channel. In CR5a, this was found to cause a variation of 0.23 events (11\%) in the $4 e$ channel and 0.38 events $(17 \%)$ in the $2 \mu 2 e$ channel.

\subsubsection{Reducible background yield in the $7 \mathrm{TeV}$ data}

The transfer factors are calculated using Equation 6.8 for CR5 and Equation 6.2 for CR5a, with the appropriate efficiencies and compositions used to calculate the efficiencies. The final results for the reducible background yields for the two control 
6. A data-driven estimation of the reducible electron-like background in the $H \rightarrow Z Z^{(*)} \rightarrow 4 \ell$ channel: the Transfer Factor method

regions are shown in Table 6.17 and Table 6.18. Comparison with simulations indicates that CR5 estimates the yield due to $W Z$ well and so, unlike in CR5a, an additional contribution is not determined and added from Monte Carlo. Due to the statistical limitations of CR5a, the background yield determined using CR5 should be considered as the primary estimate, with the yield determined using CR5a serving as a secondary estimate. Nonetheless, the two estimates do agree with one another and with the yield obtained using the baseline $3 \ell+X$ method (refer to Table 5.7), within the uncertainty.

\begin{tabular}{ccc}
\hline \hline & $4 e$ & $2 \mu 2 e$ \\
\hline Data & $2.77 \pm 0.35$ (stat.) & $2.98 \pm 0.36$ (stat.) \\
$Z Z^{(*)}(\mathrm{MC})$ & $0.16 \pm 0.00$ (stat.) & $0.19 \pm 0.00$ (stat.) \\
Final estimate & $2.61 \pm 0.35$ (stat.) \pm 0.91 (syst.) & $2.79 \pm 0.36$ (stat.) \pm 1.05 (syst.) \\
\hline Baseline estimate & $3.3 \pm 0.5$ (stat.) \pm 0.5 (syst.) & $2.9 \pm 0.5$ (stat.) \pm 0.5 (syst.) \\
\hline \hline
\end{tabular}

TABLE 6.17: The reducible background estimate for the $7 \mathrm{TeV}$ data, determined using the Transfer Factor method with CR5. The preliminary estimate is obtained from data, and the $Z Z^{(*)}$ contribution obtained by applying the method to Monte Carlo is subtracted.

\begin{tabular}{ccc}
\hline \hline & $4 e$ & $2 \mu 2 e$ \\
\hline Data & $1.75 \pm 0.28$ (stat.) & $2.00 \pm 0.32$ (stat.) \\
$Z Z^{(*)}(\mathrm{MC})$ & $0.01 \pm 0.00$ (stat.) & $0.01 \pm 0.00$ (stat.) \\
$W Z(\mathrm{MC})$ & $0.31 \pm 0.14$ (stat.) & $0.25 \pm 0.10$ (stat.) \\
Final estimate & $2.05 \pm 0.31$ (stat.) \pm 0.93 (syst.) & $2.24 \pm 0.34$ (stat.) \pm 1.06 (syst.) \\
\hline Baseline estimate & $3.3 \pm 0.5$ (stat.) \pm 0.5 (syst.) & $2.9 \pm 0.5$ (stat.) \pm 0.5 (syst.) \\
\hline \hline
\end{tabular}

TABLE 6.18: The reducible background estimate for the $7 \mathrm{TeV}$ data, determined using the Transfer Factor method with CR5a. The preliminary estimate is obtained from data, and the $Z Z^{(*)}$ contribution obtained by applying the method to Monte Carlo is subtracted. An additional contribution from the $W Z$ process is added by applying the full analysis selection on Monte Carlo to obtain the final reducible background yield in the signal region. 
6. A data-driven estimation of the reducible electron-like background in the $H \rightarrow Z Z^{(*)} \rightarrow 4 \ell$ channel: the Transfer Factor method

\subsection{Conclusion}

A data-driven method for estimating the reducible background yield due to $Z+$ jets, $Z+b \bar{b}, t \bar{t}$, and $W Z$ in the subleading electron channels of the $H \rightarrow Z Z^{(*)} \rightarrow 4 \ell$ process has been presented. This method, known as the Transfer Factor method, uses a signal-free sample of electron-like background objects to determine the probabilities for background objects to pass the selection cuts. These efficiencies are then used to extrapolate from a signal-free control region constructed using events from data to estimate the reducible background yield in the signal region.

The results of the Transfer Factor method have been validated and found to be in good agreement with the main method used for the reducible background estimation in the $H \rightarrow Z Z^{(*)} \rightarrow 4 \ell$ analysis. The final results for the background yield in the $7 \mathrm{TeV}$ and $8 \mathrm{TeV}$ data estimated using the Transfer Factor method are summarized in Table 6.19.

\begin{tabular}{ccc}
\hline \hline & $4 e$ & $2 \mu 2 e$ \\
\hline $8 \mathrm{TeV}$ & $2.45 \pm 0.10 \pm 0.89$ & $2.52 \pm 0.10 \pm 0.90$ \\
$7 \mathrm{TeV}$ (CR5) & $2.61 \pm 0.35 \pm 0.91$ & $2.79 \pm 0.36 \pm 1.05$ \\
$7 \mathrm{TeV}$ (CR5a) & $2.05 \pm 0.31 \pm 0.93$ & $2.24 \pm 0.34 \pm 1.06$ \\
\hline \hline
\end{tabular}

TABLE 6.19: Summary of the estimated reducible background yields in the $7 \mathrm{TeV}$ and $8 \mathrm{TeV}$ data determined using the Transfer Factor method. The errors correspond to the statistical and systematic uncertainties, respectively.

With a good understanding of the expected background yields, it is possible to determine the Higgs boson signal yield in the four-lepton decay channel, and to perform precision measurements of its properties using a clean signal sample. These objectives will be discussed in the next chapters. 


\title{
7. Results of the search for the Higgs bo- son in the $H \rightarrow Z Z^{(*)} \rightarrow 4 \ell$ channel
}

Who in the world am I? Ah, that's the great puzzle.

— Lewis Carroll, Alice's Adventures in

Wonderland

\begin{abstract}
S the ATLAS experiment moves on from the discovery era to the pre(t) cision measurement era, the electron identification, event selection, and background estimation methods described in the preceding chapters have all been carefully designed to maximize the signal efficiency and background rejection in order to perform the first measurements of the Higgs boson's properties.
\end{abstract}

The final results of the $7 \mathrm{TeV}$ and $8 \mathrm{TeV}$ event selection $[4,6]$ will be presented in Section 7.1. The author's major contribution to these results was the estimation of the reducible electron-like background described in Chapter 6. Distributions of several important observables will be shown in Section 7.2. These will be followed by brief summaries of the mass and signal strength measurements in Section 7.3, the categorized analysis probing the Higgs boson's production mechanisms in Section 7.4, and the spin-parity measurement in Section 7.5. 
7. Results of the search for the Higgs boson in the $H \rightarrow Z Z^{(*)} \rightarrow 4 \ell$ channel 150

\subsection{Results of the event selection}

Table 7.1 gives the observed yield in the $7 \mathrm{TeV}$ and $8 \mathrm{TeV}$ analyses, after the event selection described in Section 5.3. Table 7.2 gives the observed yield in a window of $110 \mathrm{GeV}<m_{4 \ell}<140 \mathrm{GeV}$ (the range used for the mass measurement), as well as the expected background yields in the same mass window obtained using the methods described in Section 5.4 and the expected signal yield for a Standard Model Higgs boson hypothesis with $m_{H}=125 \mathrm{GeV}$. It can be seen that the observed yield exceeds the Standard Model expectation in most channels.

\begin{tabular}{ccc}
\hline \hline & $\sqrt{s}=7 \mathrm{TeV}$ & $\sqrt{s}=8 \mathrm{TeV}$ \\
\hline $4 \mu$ & 34 & 137 \\
$2 \mu 2 e$ & 13 & 88 \\
$2 e 2 \mu$ & 18 & 124 \\
$4 e$ & 18 & 79 \\
\hline Total & 83 & 428 \\
\hline \hline
\end{tabular}

TABLE 7.1: Total observed number of events in data for the $7 \mathrm{TeV}(8 \mathrm{TeV})$ analyses, corresponding to an integrated luminosity of $4.5 \mathrm{fb}^{-1}\left(20.3 \mathrm{fb}^{-1}\right)$.

\begin{tabular}{cccccc}
\hline \hline & Signal & $Z Z^{(*)}$ & $Z+$ jets, $Z+b \bar{b}, t \bar{t}$ & Total expected & Observed \\
\hline & \multicolumn{5}{c}{$\sqrt{s}=7 \mathrm{TeV}$} \\
\hline $4 \mu$ & $0.98 \pm 0.09$ & $1.26 \pm 0.06$ & $0.26 \pm 0.12$ & $2.51 \pm 0.16$ & 4 \\
$2 \mu 2 e$ & $0.48 \pm 0.05$ & $0.58 \pm 0.04$ & $1.02 \pm 0.23$ & $2.08 \pm 0.23$ & 1 \\
$2 e 2 \mu$ & $0.64 \pm 0.06$ & $0.87 \pm 0.04$ & $0.24 \pm 0.09$ & $1.75 \pm 0.12$ & 3 \\
$4 e$ & $0.44 \pm 0.05$ & $0.51 \pm 0.03$ & $1.14 \pm 0.24$ & $2.10 \pm 0.25$ & 2 \\
Total & $2.55 \pm 0.25$ & $3.23 \pm 0.17$ & $2.66 \pm 0.50$ & $8.45 \pm 0.59$ & 10 \\
\hline \multicolumn{5}{c}{$\sqrt{s}=8 \mathrm{TeV}$} \\
\hline $4 \mu$ & $5.68 \pm 0.56$ & $6.49 \pm 0.32$ & $1.78 \pm 0.32$ & $13.95 \pm 0.73$ & 18 \\
$2 \mu 2 e$ & $2.99 \pm 0.31$ & $3.19 \pm 0.20$ & $1.01 \pm 0.24$ & $7.20 \pm 0.43$ & 10 \\
$2 e 2 \mu$ & $3.84 \pm 0.38$ & $4.50 \pm 0.22$ & $1.55 \pm 0.26$ & $9.89 \pm 0.51$ & 12 \\
$4 e$ & $2.72 \pm 0.28$ & $2.88 \pm 0.19$ & $1.00 \pm 0.21$ & $6.61 \pm 0.40$ & 10 \\
Total & $15.23 \pm 1.53$ & $17.07 \pm 0.93$ & $5.34 \pm 0.74$ & $37.64 \pm 1.94$ & 50 \\
\hline \hline
\end{tabular}

TABLE 7.2: Expected number of signal and background events, as well as the observed yield in data in the mass window $110-140 \mathrm{GeV}$ for the $7 \mathrm{TeV}$ and $8 \mathrm{TeV}$ analyses. The errors correspond to the total uncertainties. 


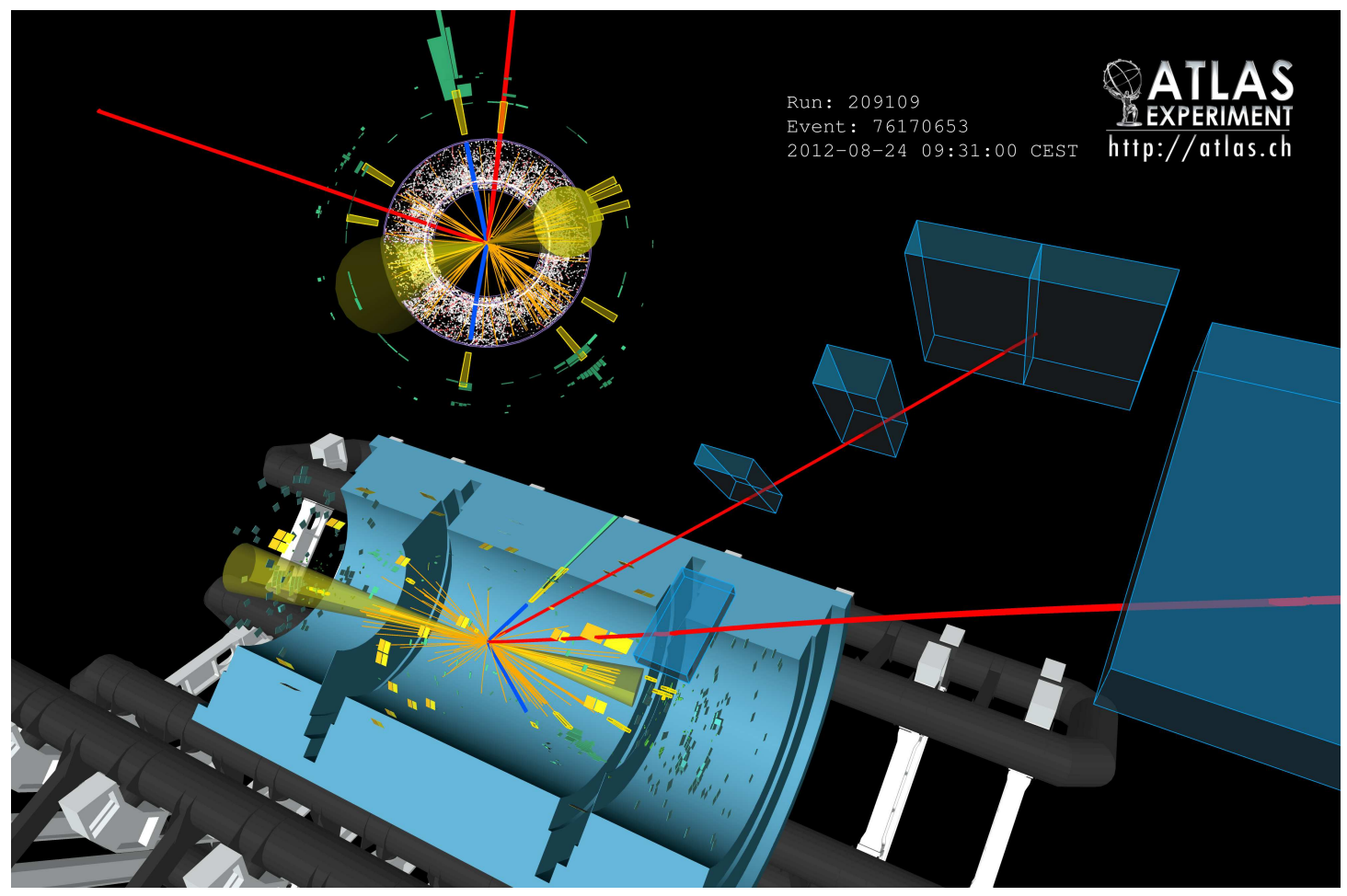

FiguRE 7.1: Event display for a $H \rightarrow Z Z^{*} \rightarrow 2 e 2 \mu$ candidate observed in the $8 \mathrm{TeV}$ data. Electron (muon) tracks are illustrated in blue (red). Electromagnetic (hadronic) calorimeter energy deposits are shown in green (yellow). This candidate has an invariant mass of $m_{4 \ell}=123.4 \mathrm{GeV}$ and satisfies the criteria for the VBF production mode category, as described in Section 7.4 [6].

Figure 7.1 is an event display for a $2 e 2 \mu$ event observed in the $8 \mathrm{TeV}$ data. Several key features of the reconstructed event, including charged particle tracks and energy deposits in the calorimeters, are illustrated. The event satisfies the criteria for the VBF production mode category, which will be described in Section 7.4.

\subsection{Distributions of key observables}

Several important observables' distributions are shown for the $7 \mathrm{TeV}$ and $8 \mathrm{TeV}$ analyses. The distributions of the selected candidate events' invariant masses 
$\left(m_{4 \ell}\right)$ are shown for both the full mass range (without any restrictions on $m_{4 \ell}$ ) in Figure 7.2, and for the low mass range $\left(80 \mathrm{GeV}<m_{4 \ell}<170 \mathrm{GeV}\right)$ in Figure 7.3.

The distributions of several additional observables are shown in the range used for the mass measurement $\left(110 \mathrm{GeV}<m_{4 \ell}<140 \mathrm{GeV}\right)$; these distributions include the leading dilepton mass $\left(m_{12}\right)$ in Figure 7.4, the subleading dilepton mass $\left(m_{34}\right)$ in Figure 7.5 , the transverse momentum $\left(p_{\mathrm{T}, 4 \ell}\right)$ in Figure 7.6, the pseudorapidity $\left(\eta_{4 \ell}\right)$ in Figure 7.7, and in Figure 7.8, the BDT discriminant introduced in Section 5.4.1.

For each observable, the data distribution is accompanied by the distributions of the irreducible and reducible backgrounds, as well as the expected distribution for a Higgs boson with $m_{H}=125 \mathrm{GeV}$, normalized to the signal strength $\mu=1.66$, which is defined as the ratio of the observed signal to the Standard Model expectation (this parameter will be discussed in more detail in Section 7.3).

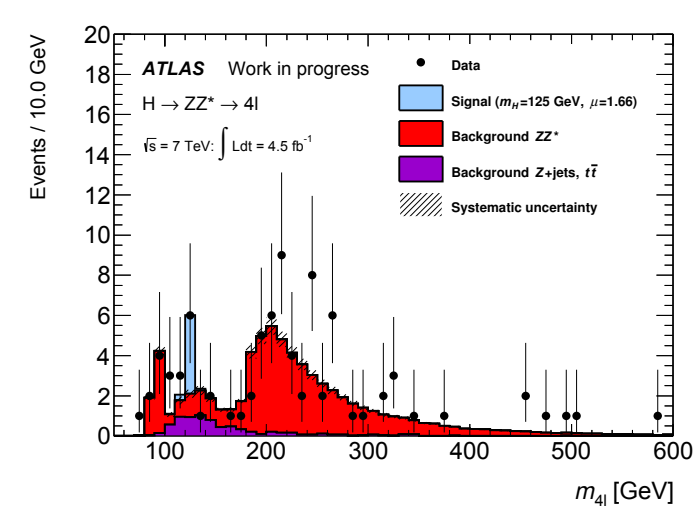

(a) $\sqrt{s}=7 \mathrm{TeV}$

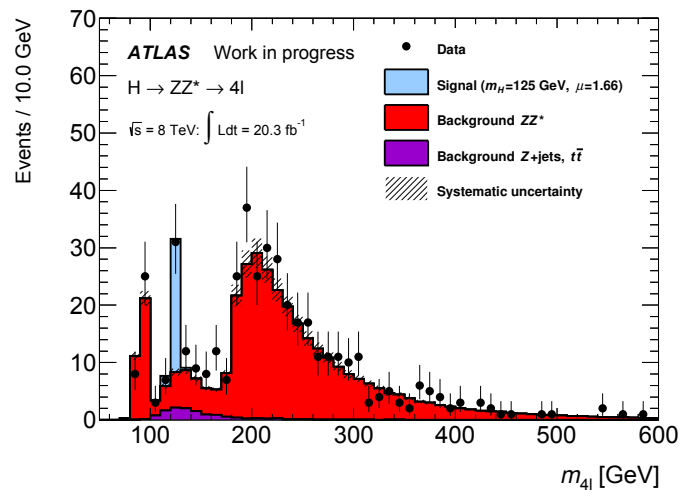

(b) $\sqrt{s}=8 \mathrm{TeV}$

Figure 7.2: Four-lepton invariant mass $\left(m_{4 \ell}\right)$ distributions in the $7 \mathrm{TeV}$ and $8 \mathrm{TeV}$ data for the full mass range. Also shown are the expected distributions for the backgrounds and a Standard Model Higgs boson with $m_{H}=125 \mathrm{GeV}$, normalized to the measured signal strength $\mu=1.66$. 


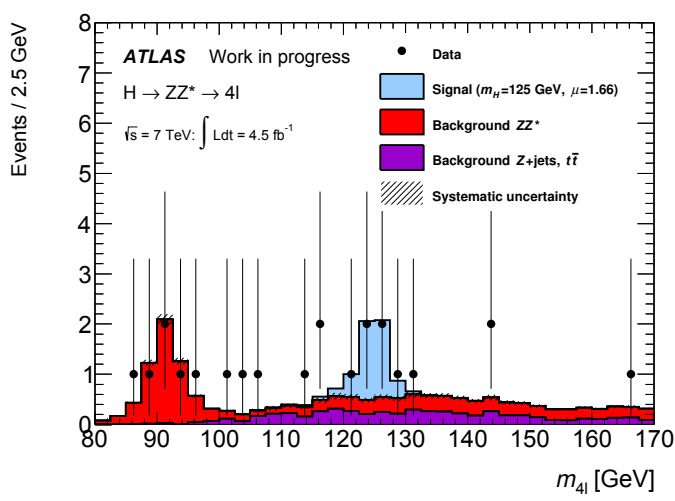

(a) $\sqrt{s}=7 \mathrm{TeV}$

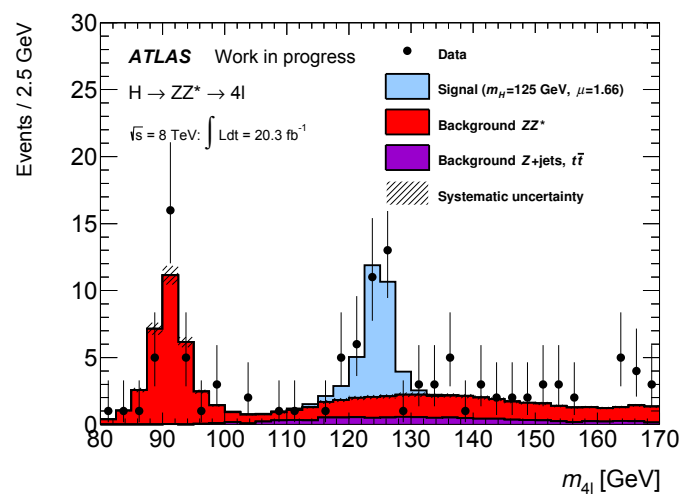

(b) $\sqrt{s}=8 \mathrm{TeV}$

FIgURE 7.3: Four-lepton invariant mass $\left(m_{4 \ell}\right)$ distributions in the $7 \mathrm{TeV}$ and $8 \mathrm{TeV}$ data for the low mass range. Also shown are the expected distributions for the backgrounds and a Standard Model Higgs boson with $m_{H}=125 \mathrm{GeV}$, normalized to the measured signal strength $\mu=1.66$.

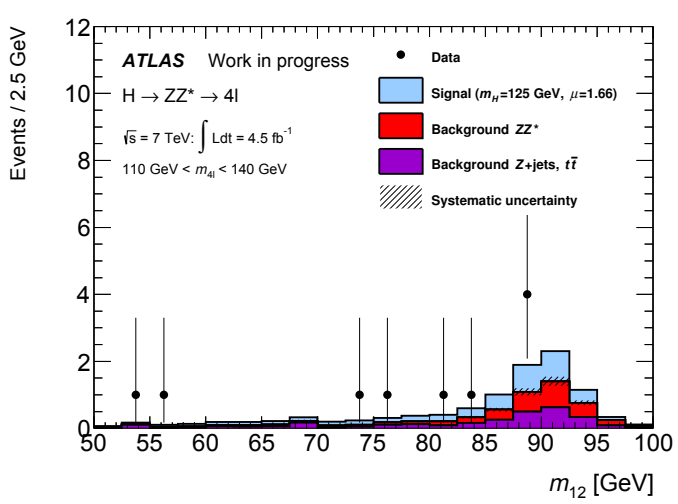

(a) $\sqrt{s}=7 \mathrm{TeV}$

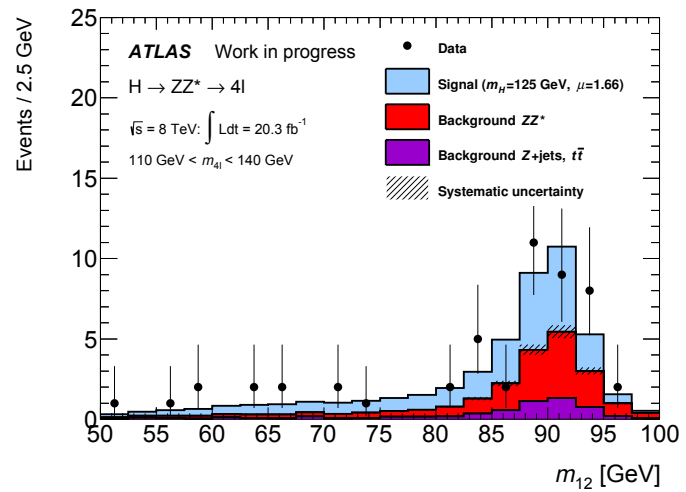

(b) $\sqrt{s}=8 \mathrm{TeV}$

Figure 7.4: Leading dilepton invariant mass $\left(m_{12}\right)$ distributions in the $7 \mathrm{TeV}$ and $8 \mathrm{TeV}$ data for the mass window $110 \mathrm{GeV}<m_{4 \ell}<140 \mathrm{GeV}$. Also shown are the expected distributions for the backgrounds and a Standard Model Higgs boson with $m_{H}=125 \mathrm{GeV}$, normalized to the measured signal strength $\mu=$ 1.66 . 


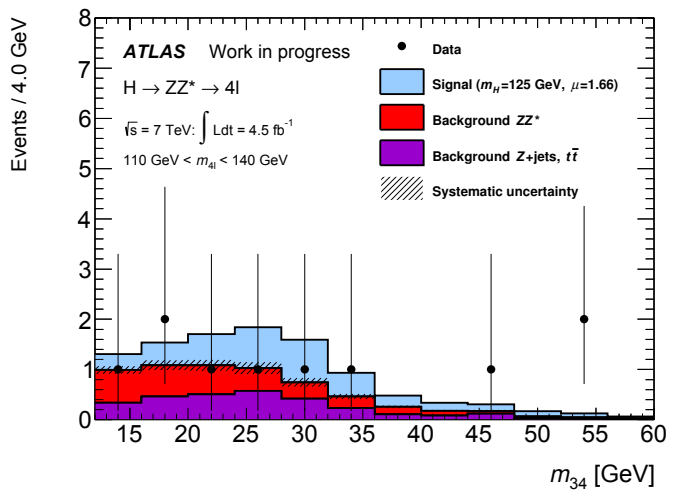

(a) $\sqrt{s}=7 \mathrm{TeV}$

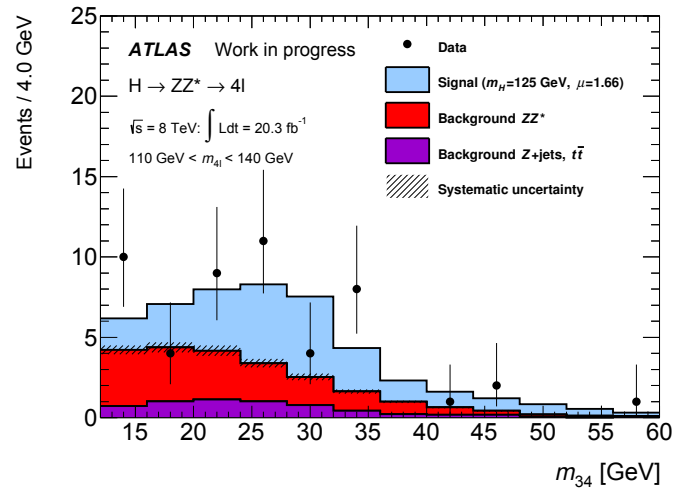

(b) $\sqrt{s}=8 \mathrm{TeV}$

FiguRE 7.5: Subleading dilepton invariant mass $\left(m_{34}\right)$ distributions in the $7 \mathrm{TeV}$ and $8 \mathrm{TeV}$ data for the mass window $110 \mathrm{GeV}<m_{4 \ell}<140 \mathrm{GeV}$. Also shown are the expected distributions for the backgrounds and a Standard Model Higgs boson with $m_{H}=125 \mathrm{GeV}$, normalized to the measured signal strength $\mu=1.66$.

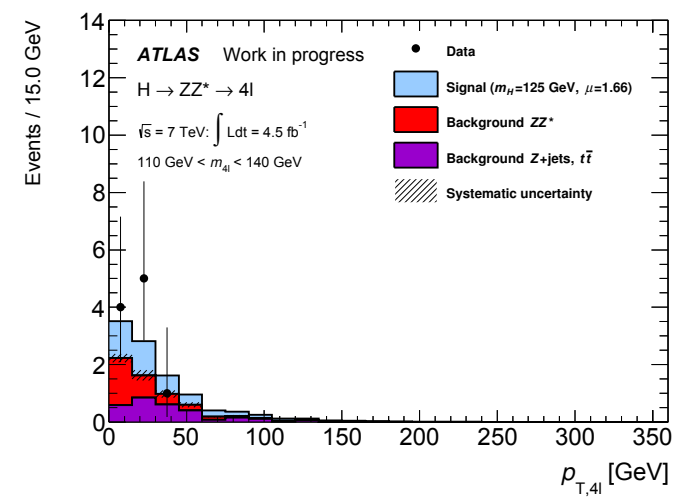

(a) $\sqrt{s}=7 \mathrm{TeV}$

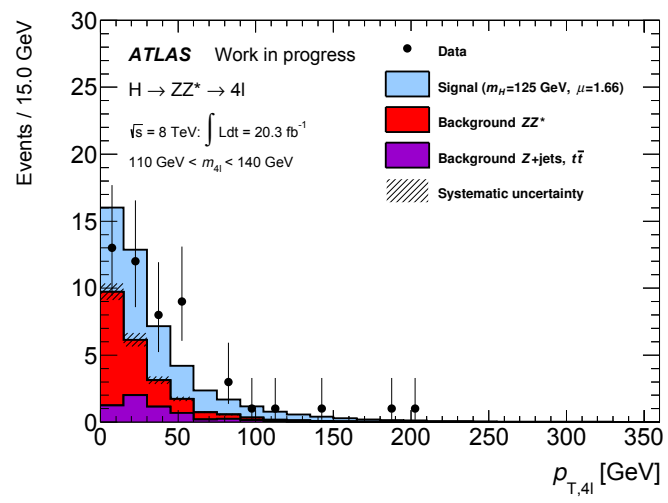

(b) $\sqrt{s}=8 \mathrm{TeV}$

FiguRE 7.6: Four-lepton transverse momentum $\left(p_{\mathrm{T}, 4 \ell}\right)$ distributions in the $7 \mathrm{TeV}$ and $8 \mathrm{TeV}$ data for the mass window $110 \mathrm{GeV}<m_{4 \ell}<140 \mathrm{GeV}$. Also shown are the expected distributions for the backgrounds and a Standard Model Higgs boson with $m_{H}=125 \mathrm{GeV}$, normalized to the measured signal strength $\mu=1.66$. 


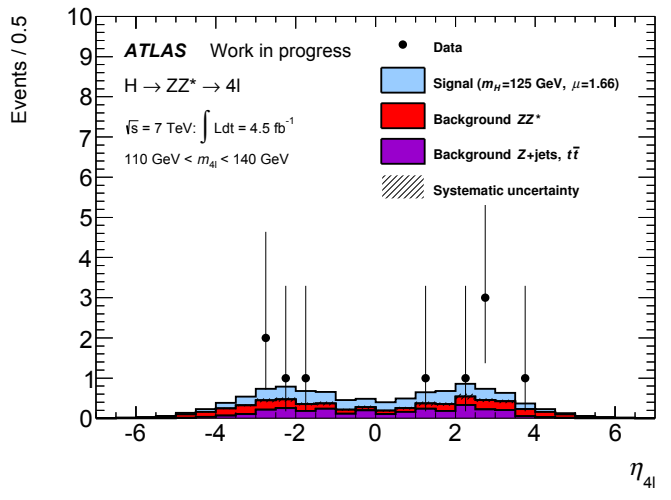

(a) $\sqrt{s}=7 \mathrm{TeV}$

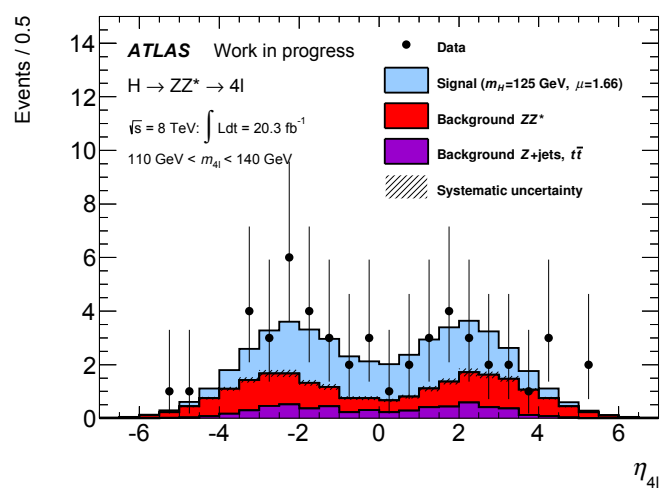

(b) $\sqrt{s}=8 \mathrm{TeV}$

FiguRE 7.7: Four-lepton pseudorapidity $\left(\eta_{4 \ell}\right)$ distributions in the $7 \mathrm{TeV}$ and $8 \mathrm{TeV}$ data for the mass window $110 \mathrm{GeV}<m_{4 \ell}<140 \mathrm{GeV}$. Also shown are the expected distributions for the backgrounds and a Standard Model Higgs boson with $m_{H}=125 \mathrm{GeV}$, normalized to the measured signal strength $\mu=1.66$.

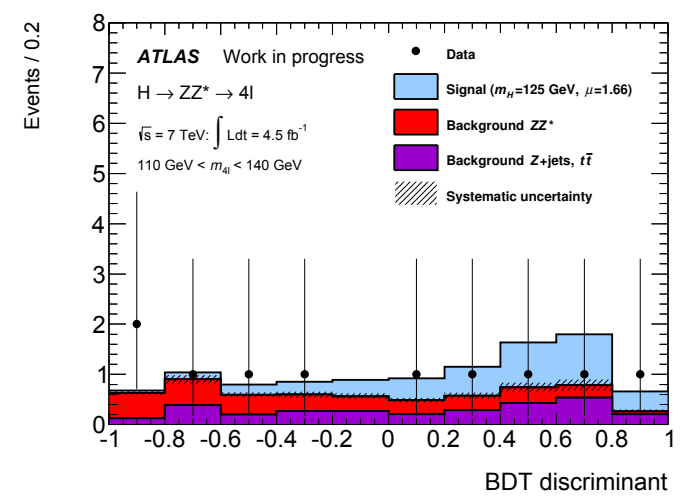

(a) $\sqrt{s}=7 \mathrm{TeV}$

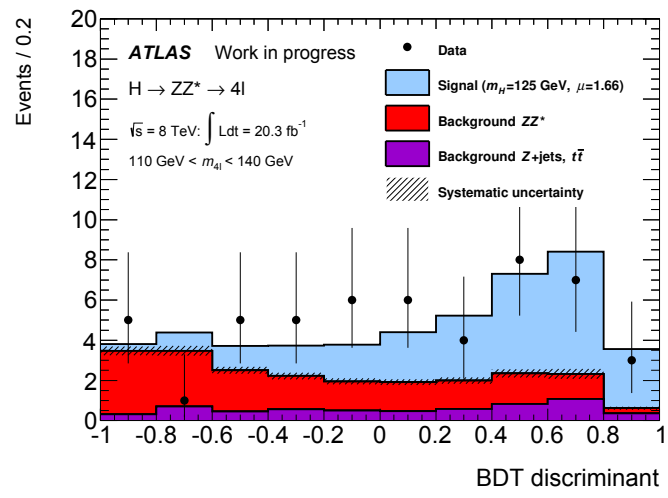

(b) $\sqrt{s}=8 \mathrm{TeV}$

Figure 7.8: BDT discriminant distributions in the $7 \mathrm{TeV}$ and $8 \mathrm{TeV}$ data for the mass window $110 \mathrm{GeV}<m_{4 \ell}<140 \mathrm{GeV}$. Also shown are the expected distributions for the backgrounds and a Standard Model Higgs boson with $m_{H}=$ $125 \mathrm{GeV}$, normalized to the measured signal strength $\mu=1.66$. 


\subsection{Measurement of the mass and signal strength}

The mass measurement [4] is performed using all candidates from the $7 \mathrm{TeV}$ and $8 \mathrm{TeV}$ analyses having invariant masses in the range $110 \mathrm{GeV}<m_{4 \ell}<$ $140 \mathrm{GeV}$. The baseline method for the mass measurement involves performing a two-dimensional fit to the $m_{4 \ell}$ distribution and the BDT output, which provides additional discrimination against the irreducible $Z Z^{(*)}$ background. This twodimensional distribution is shown in Figure 7.9. For the fit, the signal is modelled in the range $115 \mathrm{GeV}<m_{H}<130 \mathrm{GeV}$ using smoothed shapes from simulations, parameterized as a function of $m_{H}$. The $Z Z^{(*)}$ background and reducible $Z+\mu \mu$ background shapes are also modelled using simulations. The reducible $Z+e e$ background shape is taken from data since this background is dominated by the $Z+$ jets process, which is not well-modelled by simulations.

\subsubsection{Systematic uncertainties}

The main sources of systematic uncertainty on the mass measurement are the uncertainties on the electron energy scale and the muon momentum scale. Varying the energy and momentum scales by their respective uncertainties yields contributions to the systematic uncertainty on $m_{H}$ of $0.03 \%$ for each scale. The uncertainty on the luminosity measurement corresponds to $\pm 1.8 \%$ for the $7 \mathrm{TeV}$ data [35] and $\pm 2.8 \%$ for the $8 \mathrm{TeV}$ data; the effect of this uncertainty on $m_{H}$ is negligible.

The sources of systematic uncertainty on the signal strength measurement are summarized in Table 7.3. Experimental sources of systematic uncertainty include the trigger, lepton reconstruction, and identification efficiencies. The difference in agreement between data and simulation of the efficiency of the isolation and 


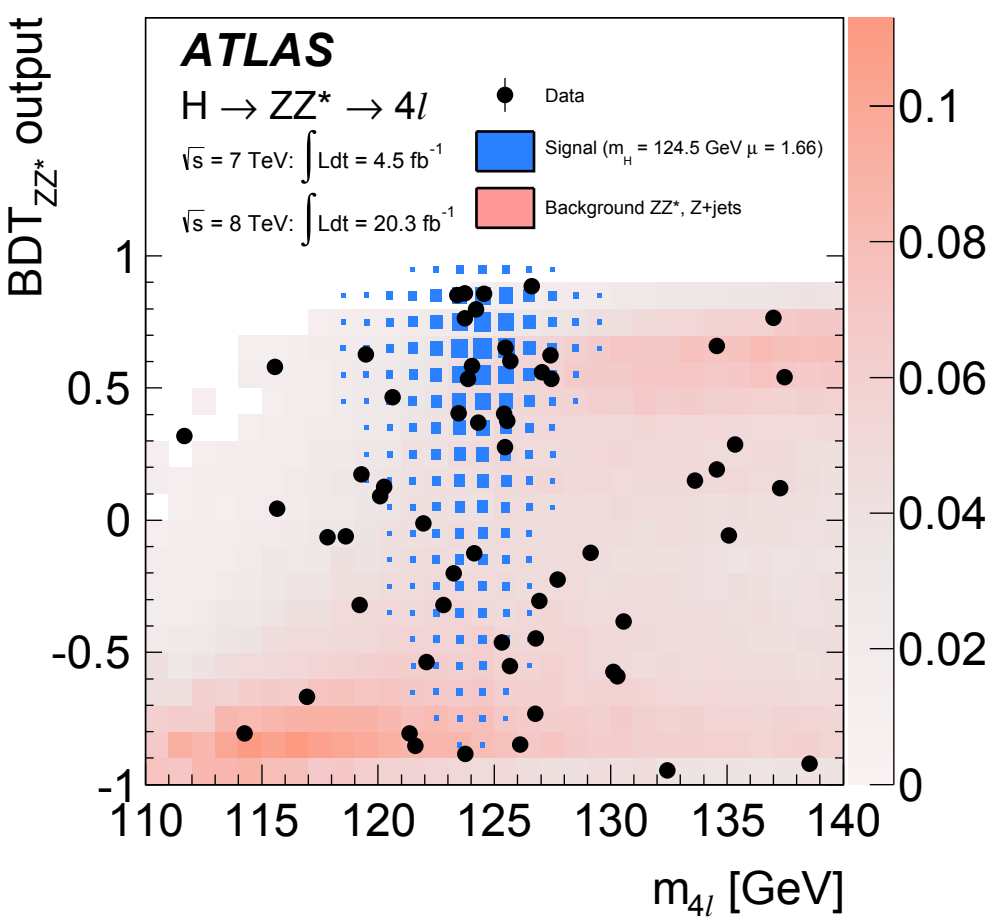

Figure 7.9: Distribution of the BDT discriminant versus $m_{4 \ell}$ in the $7 \mathrm{TeV}$ and $8 \mathrm{TeV}$ data for the mass window $110 \mathrm{GeV}<m_{4 \ell}<140 \mathrm{GeV}$. Also shown are the expected distributions for the backgrounds and for a Standard Model Higgs boson with $m_{H}=125 \mathrm{GeV}$, normalized to the measured signal strength $\mu=1.66$.

impact parameter significance cuts contributes a small uncertainty for channels involving electrons. The systematic uncertainties due to the data-driven background estimates were discussed in Chapter 5. Theoretical uncertainties on the signal and background models include the QCD scale, PDF, and $\alpha_{s}$ uncertainties, which are evaluated by considering the variation between several sets of PDFs calculated using different datasets and analysis frameworks as described in [113], as well as the uncertainty on the $H \rightarrow Z Z^{*}$ branching ratio.

The total systematic uncertainty on the mass and signal strength measurements is determined by subtracting in quadrature the uncertainty of the fits evaluated with and without the systematic uncertainties. 
7. Results of the search for the Higgs boson in the $H \rightarrow Z Z^{(*)} \rightarrow 4 \ell$ channel 158

\begin{tabular}{lccccc}
\hline \hline Source of uncertainty & $4 \mu$ & $2 e 2 \mu$ & $2 \mu 2 e$ & $4 e$ & Combined \\
\hline Electron reconstruction and identification efficiencies & - & $1.7 \%$ & $3.3 \%$ & $4.4 \%$ & $1.6 \%$ \\
Electron isolation and impact parameter significance & - & $0.07 \%$ & $1.1 \%$ & $1.2 \%$ & $0.5 \%$ \\
Electron trigger efficiency & - & $0.21 \%$ & $0.05 \%$ & $0.21 \%$ & $<0.2 \%$ \\
$Z+e e$ background & - & - & $3.4 \%$ & $3.4 \%$ & $1.3 \%$ \\
\hline Muon reconstruction and identification efficiencies & $1.9 \%$ & $1.1 \%$ & $0.8 \%$ & - & $1.5 \%$ \\
Muon trigger efficiency & $0.6 \%$ & $0.03 \%$ & $0.6 \%$ & - & $0.2 \%$ \\
$Z+\mu \mu$ background & $1.6 \%$ & $1.6 \%$ & - & - & $1.2 \%$ \\
\hline QCD scale & & & & & $6.5 \%$ \\
PDF,$\alpha_{s}$ & & & & & $6.0 \%$ \\
$H \rightarrow Z Z^{*}$ branching ratio & & & & $4.0 \%$ \\
\hline \hline
\end{tabular}

TABLE 7.3: The main sources of systematic uncertainty on the signal strength measurement.

\subsubsection{Results of the mass and signal strength measure- ments}

The result of the mass measurement using the combined $7 \mathrm{TeV}$ and $8 \mathrm{TeV}$ data corresponds to:

$$
m_{H}=124.51 \pm 0.52 \text { (stat.) } \pm 0.06 \text { (syst.) GeV }
$$

The local $p_{0}$, which represents the probability that the observed excess is due to fluctuations in the background, is shown for the $7 \mathrm{TeV}, 8 \mathrm{TeV}$, and combined datasets in Figure 7.10. The solid lines correspond to the oberved signal, while the dashed lines correspond to the expectation based on the background-only hypothesis. The observed excess corresponds to a significance of 8.2 standard deviations at the measured Higgs boson mass.

The signal strength, defined as the ratio of the observed production rate to the Standard Model expectation, is measured to be:

$$
\mu=1.66_{-0.34}^{+0.39} \text { (stat.) }{ }_{-0.14}^{+0.21} \text { (syst.), }
$$




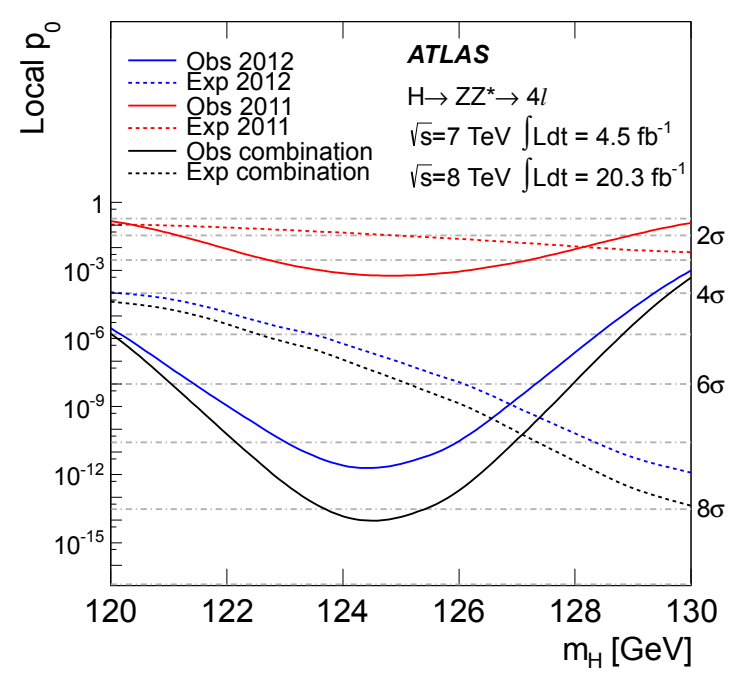

(a) Local $p_{0}$

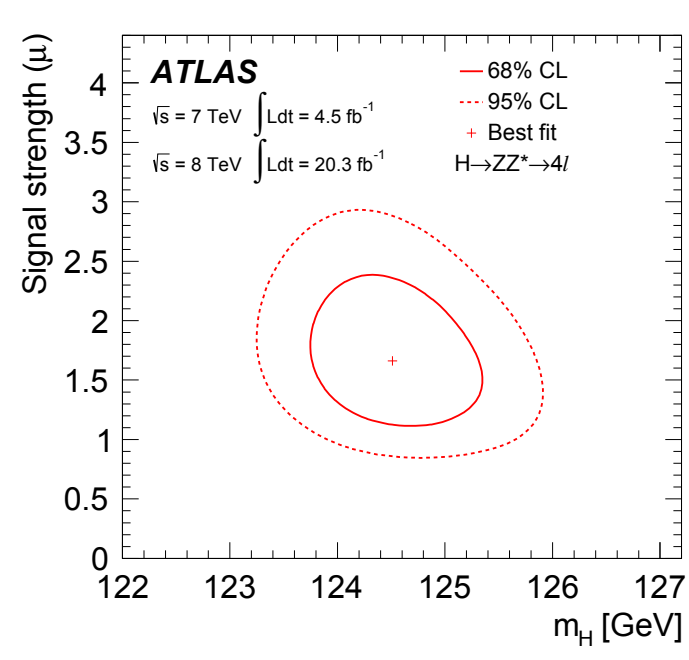

(b) Signal strength

FiguRe 7.10: The observed local $p_{0}$ and signal strength as a function of $m_{H}$ for the $7 \mathrm{TeV}$ and $8 \mathrm{TeV}$ datasets. At the combined dataset's best-fit mass of $m_{H}=$ $124.51 \pm 0.52$ (stat.) \pm 0.06 (syst.) $\mathrm{GeV}$, the local $p_{0}$ corresponds to an observed significance of 8.2 standard deviations above the background-only hypothesis. The signal strength at the best fit mass is $\mu=1.66_{-0.34}^{+0.39}$ (stat.) ${ }_{-0.14}^{+0.21}$ (syst.), which is consistent with the Standard Model expectation of $\mu=1$ [6].

which is slightly high, but still consistent with the Standard Model expectation of $\mu=1$. The signal strength as a function of $m_{H}$ is shown in Figure 7.10.

The result of the $H \rightarrow Z Z^{(*)} \rightarrow 4 \ell$ mass measurement has been combined with that obtained using the $H \rightarrow \gamma \gamma$ channel [114] in order to produce the final ATLAS Run I result [4]:

$$
m_{H}=125.36 \pm 0.37 \text { (stat.) } \pm 0.18 \text { (syst.) GeV }
$$

For comparison purposes, the CMS collaboration has measured the Higgs boson's mass in the $H \rightarrow Z Z^{(*)} \rightarrow 4 \ell$ channel as $m_{H}=125.6 \pm 0.4$ (stat.) \pm 0.2 (syst.) $\mathrm{GeV}$; the signal strength at the best-fit mass in this channel is $\mu=$ $0.93_{-0.23}^{+0.26}$ (stat.) ${ }_{-0.09}^{+0.13}$ (syst.) [115]. The combined CMS result, including the 
$H \rightarrow \gamma \gamma$ channel, is $m_{H}=125.02_{-0.27}^{+0.26}$ (stat.) ${ }_{-0.15}^{+0.14}$ (syst.) $\mathrm{GeV}[116]$; the ATLAS and CMS results are consistent with one another, within the experimental uncertainties.

\subsection{Event categorization}

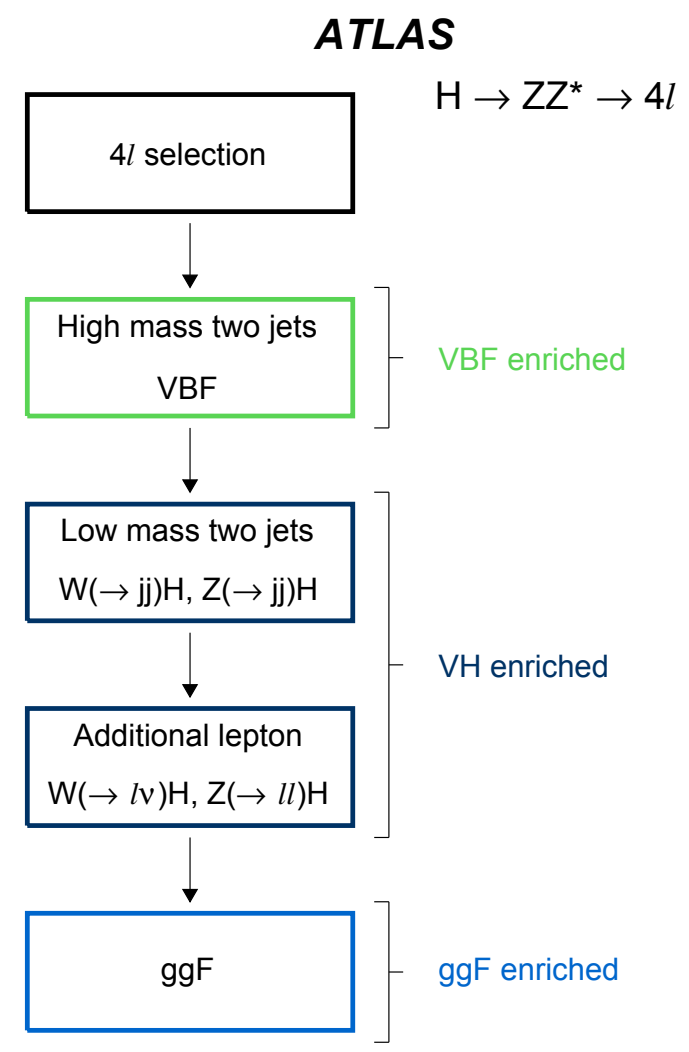

FiguRe 7.11: Schematic of the procedure for assigning candidate events to categories corresponding to the various Higgs boson production modes [6].

In order to investigate whether the observed event yields agree with theoretical predictions corresponding to the Higgs boson's production modes and couplings, the selected candidate events are divided into four categories corresponding to the production modes discussed in Chapter 2: vector boson fusion 
7. Results of the search for the Higgs boson in the $H \rightarrow Z Z^{(*)} \rightarrow 4 \ell$ channel 161

(VBF), associated production with a $W$ or $Z$ boson $(\mathrm{VH})$, where the vector boson can decay either hadronically or leptonically, and gluon fusion (ggF). The events are categorized sequentially; if an event fails the criteria for one category, the criteria for the next category are then checked. This process is depicted schematically in Figure 7.11. The categorization criteria are defined as follows:

- VBF-enriched Events must have two high- $p_{\mathrm{T}}$ jets satisfying $p_{\mathrm{T}}>25(30)$ $\mathrm{GeV}$ in the range $|\eta|<2.5(2.5<|\eta|<4.5)$. If more than two jets satisfy these criteria, the two jets having the highest $p_{\mathrm{T}}$ are chosen. The invariant mass of the dijet system must satisfy $m_{j j}>130 \mathrm{GeV}$ in order to reduce the multijet background contamination.

- VH hadronic-enriched This category also requires two high- $p_{\mathrm{T}}$ jets (as for the VBF-enriched category, the two highest- $p_{\mathrm{T}}$ jets are chosen if more than two jets are present), but they must satisfy the mass requirement $40 \mathrm{GeV}<$ $m_{j j}<130 \mathrm{GeV}$ since they are the decay products of a $W$ or $Z$ boson. A boosted decision tree (BDT) is used for further discriminating power against ggF events. Five variables are used to train the BDT: $m_{j j}$, the separation of the two jets in $|\eta|\left(\left|\Delta \eta_{j j}\right|\right)$, the $p_{\mathrm{T}}$ of each jet, and the position of the leading jet in $\eta$. The BDT discriminant must be greater than zero.

- VH leptonic-enriched At least one extra lepton, having $p_{\mathrm{T}}>8 \mathrm{GeV}$ and satisfying the lepton selection cuts, is required.

- ggF-enriched All events not falling into one of the previous three categories are assigned to this category.

The expected signal and background yields, as well as the observed yields for the combined $7 \mathrm{TeV}$ and $8 \mathrm{TeV}$ datasets are presented in Table 7.4 for the 
mass range $120 \mathrm{GeV}<m_{4 \ell}<130 \mathrm{GeV}$. Three VBF candidates are found in this range. When the candidates' BDT scores are considered $(B D T>0)$, only one likely VBF candidate, with $m_{4 \ell}=123.4 \mathrm{GeV}$, remains, in fair agreement with the expectation. No VH candidates are observed.

\begin{tabular}{|c|c|c|c|c|c|c|c|c|}
\hline \multirow[b]{2}{*}{ Category } & \multicolumn{4}{|c|}{ Signal } & \multicolumn{2}{|c|}{ Background } & \multirow{2}{*}{$\begin{array}{c}\text { Total } \\
\text { expected }\end{array}$} & \multirow[t]{2}{*}{ Observed } \\
\hline & $g g F+b \bar{b} H+t \bar{t} H$ & VBF & VH hadronic & VH leptonic & $Z Z^{(*)}$ & $Z+$ jets, $t \bar{t}$ & & \\
\hline $\mathrm{VBF}$ & $1.18 \pm 0.37$ & $0.75 \pm 0.04$ & $0.083 \pm 0.006$ & $0.013 \pm 0.001$ & $0.17 \pm 0.03$ & $0.25 \pm 0.14$ & $2.4 \pm 0.4$ & 3 \\
\hline $\operatorname{VBF}(\mathrm{BDT}>0)$ & $0.48 \pm 0.15$ & $0.62 \pm 0.04$ & $0.023 \pm 0.002$ & $0.004 \pm 0.001$ & $0.06 \pm 0.01$ & $0.10 \pm 0.05$ & $1.26 \pm 0.15$ & 1 \\
\hline VH hadronic & $0.40 \pm 0.12$ & $0.034 \pm 0.004$ & $0.20 \pm 0.01$ & $0.009 \pm 0.001$ & $0.09 \pm 0.01$ & $0.09 \pm 0.04$ & $0.80 \pm 0.12$ & 0 \\
\hline VH leptonic & $0.013 \pm 0.002$ & $<0.001$ & $<0.001$ & $0.069 \pm 0.004$ & $0.015 \pm 0.002$ & $0.016 \pm 0.019$ & $0.11 \pm 0.02$ & 0 \\
\hline ggF & $12.8 \pm 1.3$ & $0.57 \pm 0.02$ & $0.24 \pm 0.01$ & $0.11 \pm 0.01$ & $7.1 \pm 0.2$ & $2.7 \pm 0.4$ & $23.5 \pm 1.4$ & 34 \\
\hline
\end{tabular}

TABLE 7.4: Expected and observed signal and background yields for the categorized analysis of the $7 \mathrm{TeV}$ and $8 \mathrm{TeV}$ data in the mass window $120 \mathrm{GeV}<$ $m_{4 \ell}<130 \mathrm{GeV}$.

\subsubsection{Systematic uncertainties}

Uncertainties on the expected categorized event yields include the uncertainties on the theoretically predicted cross-sections for the various production modes. For categories involving jet signatures (i.e. the VBF and VH-hadronic categories), the modelling of the underlying event also contributes to the theoretical systematic uncertainty. The largest source of experimental uncertainty is the jet energy scale, which results in an uncertainty of about $10 \%$ on the yield in the VBF-enriched category, $8 \%$ in the VH-hadronic category, $1.5 \%$ in the VH-leptonic category, and $1.5 \%$ in the ggF-enriched category.

\subsubsection{Results of the categorized analysis}

In order to investigate the Higgs boson's couplings to vector bosons and fermions, the production mechanisms are grouped into bosonic $(\mathrm{VBF}+\mathrm{VH})$ and fermionic $(\mathrm{ggF}+b \bar{b} H+t \bar{t} H)$ categories, and the signal strengths, scaled by the ratio of the 
branching ratio to the Standard Model branching ratio, are determined for each category. The resulting values, obtained from the best fit to the data, are:

$$
\begin{gathered}
\mu_{\mathrm{ggF}+b \bar{b} H+t \bar{t} H} \times B / B_{\mathrm{SM}}=1.66_{-0.41}^{+0.45} \text { (stat.) }{ }_{-0.15}^{+0.25} \text { (syst.) } \\
\mu_{\mathrm{VBF}+\mathrm{VH}} \times B / B_{\mathrm{SM}}=0.26_{-0.91}^{+1.60}\left(\text { stat. }{ }_{-0.23}^{+0.36}\right. \text { (syst.). }
\end{gathered}
$$

The results of the categorized analysis are in good agreement with the Standard Model expectation for the various production modes.

\subsection{Spin-parity measurement}

According to the Standard Model, the Higgs boson should be a scalar (i.e. spin0), and should be CP-even (i.e. invariant under charge conjugation and parity inversion). The first measurements of the spin and parity of the Higgs boson have been performed [117].

A wide assortment of observables are sensitive to spin and parity; these include the masses of the two $Z$ bosons, $m_{12}$ and $m_{34}$. Five angles, pictured in Figure 7.12 and corresponding to the $Z$ boson production and decay are also considered; these angles are defined in the four-lepton rest frame. $\theta^{*}$ is the production angle of the leading $Z$ boson, $Z_{1}$. The four decay angles are: $\Phi$, the angle between the decay planes of the four leptons; $\Phi_{1}$, the angle between the decay plane of the leading $Z$ and the plane defined by the direction of $Z_{1}$ and the path of the incoming parton along the positive $z$-axis; and $\theta_{1}\left(\theta_{2}\right)$, the angle between the direction of $Z_{1}\left(Z_{2}\right)$ and its negatively-charged decay product. For the Standard 


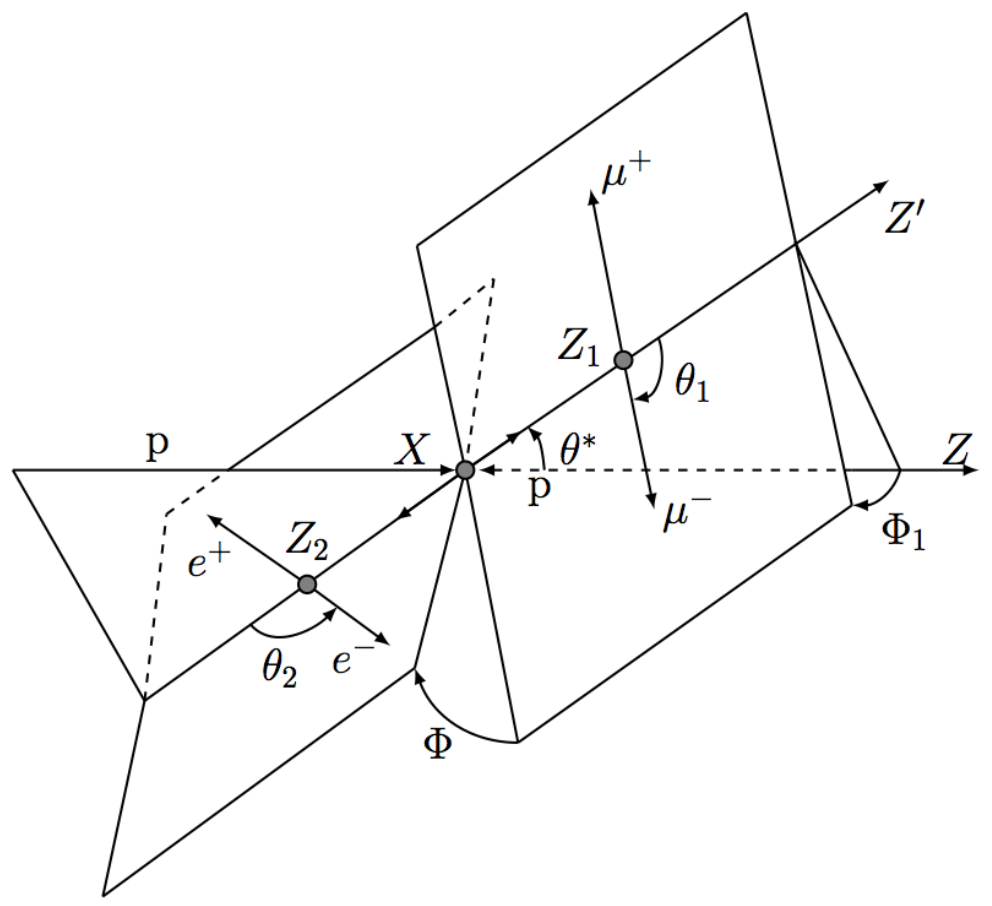

FiguRE 7.12: Schematic of the production and decay angles for a particle $X$ produced in $p-p$ collisions and decaying to two $Z$ bosons, which then decay to four leptons [5]. The leading (subleading) $Z$ boson is denoted by $Z_{1}\left(Z_{2}\right)$. The production and decay angles (described in detail in the text) are defined in the four-lepton rest frame. The beam axis is denoted by $Z$, while the positive $Z^{\prime}$ axis indicates the direction of $Z_{1}$ production.

Model hypothesis of a Higgs boson with spin-CP denoted by $J^{P}=0^{+}$, the production cross-section is independent of $\theta^{*}$ and $\Phi_{1}$, while the distributions of the other observables vary for other spin-parity hypotheses.

Two methods are used to test the spin-parity hypotheses $0^{ \pm}, 1^{ \pm}$, and $2^{ \pm}$. Since the $H \rightarrow \gamma \gamma$ decay has also been observed [2], and a particle with spin 1 cannot decay to two massless spin-1 particles, the spin-1 hypotheses are disfavoured, but they are tested for completeness. The first method uses a BDT trained using simulated events. The second method, a matrix element-based likelihood analysis (MELA) [118], uses the theoretical differential decay rate, corrected for detector and analysis acceptance, to calculate the probability of particular spin-parity 
states for each event. Both methods compare pairs of spin-parity hypotheses for candidate events in the range $115 \mathrm{GeV}<m_{4 \ell}<130 \mathrm{GeV}$. Examples of the BDT and MELA discriminants for several spin-parity hypotheses are shown in Figure 7.13 and Figure 7.14.

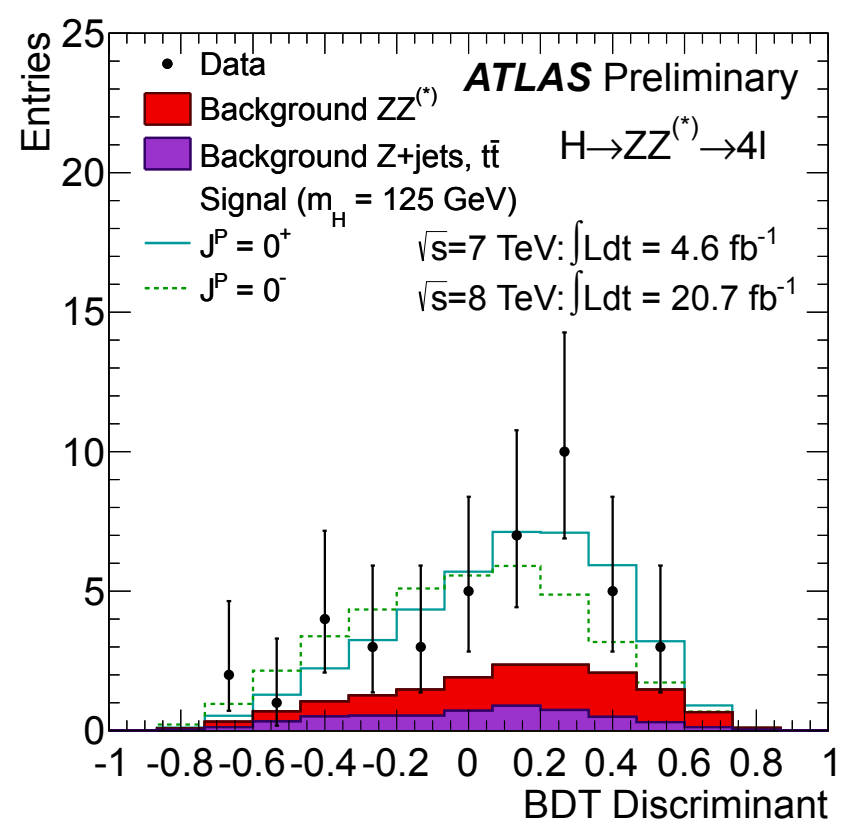

Figure 7.13: BDT discriminant for the $J^{P}=0^{+}$and $0^{-}$hypotheses [5].

As Figure 7.13 and Figure 7.14 suggest, the data favour the $J^{P}=0^{+}$ hypothesis. The log-likelihood ratios are computed for the BDT and MELA discriminants, and the non-Standard Model hypotheses are compared against the Standard Model $J^{P}=0^{+}$hypothesis. Depending on the discriminant used, the $0^{-}, 1^{+}, 1^{-}$, and $2^{+}$hypotheses are excluded at the $99.6 \%, 99.4 \%, 94.0 \%$, and $81.8 \%$ or higher confidence levels. The results of the comparison of the $0^{+}$and $2^{-}$ hypotheses are inconclusive. 


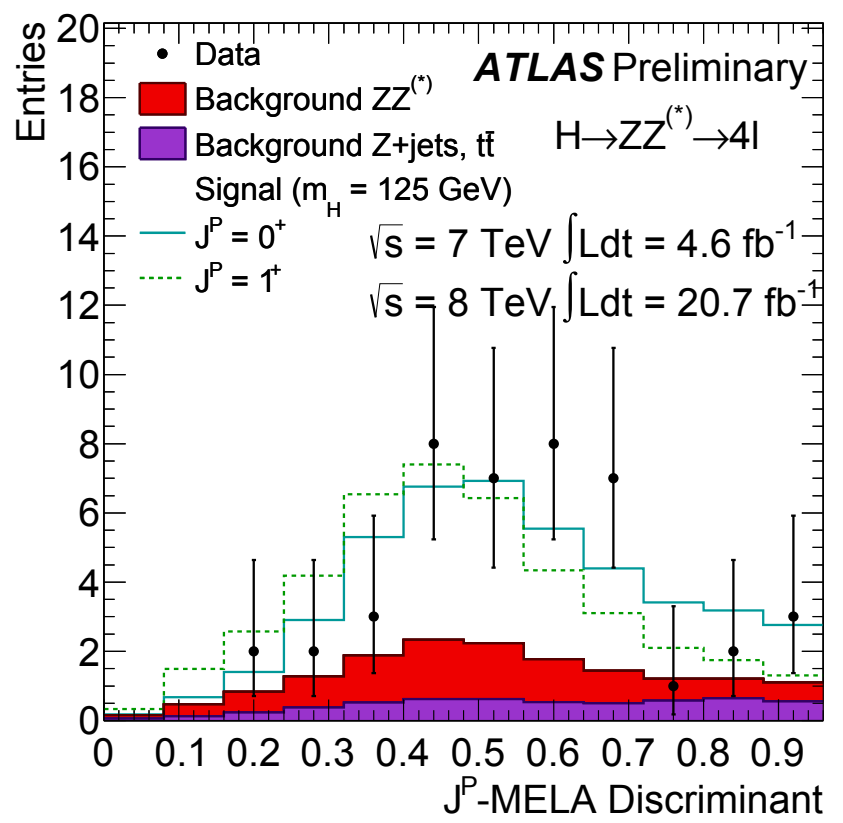

Figure 7.14: MELA discriminant for the $J^{P}=0^{+}$and $1^{+}$hypotheses [5].

\subsection{Conclusion}

Following the discovery of the Higgs boson in 2012, the first measurements of its properties in the $H \rightarrow Z Z^{(*)} \rightarrow 4 \ell$ decay channel have been performed with the full $7 \mathrm{TeV}$ and $8 \mathrm{TeV}$ datasets, amounting to approximately $20.5 \mathrm{fb}^{-1}$. The measured mass of the Higgs boson in this channel is $m_{H}=124.51 \pm 0.52$ (stat.) \pm 0.06 (syst.) $\mathrm{GeV}$ and the signal strength is $\mu=1.66_{-0.34}^{+0.39}$ (stat.) ${ }_{-0.14}^{+0.21}$ (syst.). The first candidate VBF event has been observed, providing the first tests of the Higgs boson's production modes and couplings, and the spin and parity are consistent with the $J^{P}=0^{+}$hypothesis. All of the results presented in this chapter are consistent with the hypothesis of a Standard Model Higgs boson being observed at the LHC. 


\section{Measurement of the inclusive and dif- ferential fiducial cross-sections in the $H \rightarrow Z Z^{(*)} \rightarrow 4 \ell$ channel}

If your result needs a statistician then you should design a better experiment.

— Ernest Rutherford

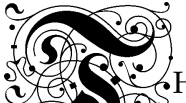
Smance of the LHC, has made it possible to graduate from the discovery era to the precision measurement era. Numerous first measurements of the Higgs boson's properties have been made; one such measurement is that of the inclusive and differential fiducial cross-sections in the $H \rightarrow Z Z^{(*)} \rightarrow 4 \ell$ channel [7], which can provide insight into the mechanisms whereby the Higgs boson is produced, and whether it conforms to the predictions of the Standard Model.

The differential cross-section is measured for six observables, which are sensitive to a wide range of properties; these will be introduced in Section 8.1. Section 8.2 defines the fiducial region in which the measurements are performed, and 
8. Measurement of the inclusive and differential fiducial cross-sections in the $H \rightarrow Z Z^{(*)} \rightarrow 4 \ell$ channel

Section 8.3 outlines the method used to extract the cross-sections from the measured signal yield and describes a study of the model-dependence of this method performed by the author of this thesis. The results are presented in Section 8.4. An alternative method, investigated by the author of this thesis, for determining the differential cross-section, which compensates for a drawback of the nominal method, is described in Section 8.5 and its future prospects are discussed in Section 8.6.

\subsection{Definition of the cross-section and observ- ables of interest}

The cross-section is, in essence, the probability that colliding particles will interact to produce a given result; in this case, protons collide to produce a Higgs boson, which subsequently decays to four leptons. For a process involving a particle (i.e. the Higgs boson) being produced in collisions and then decaying to a final state, the cross-section $\sigma$ (typically measured in units of $\mathrm{fb}$ ) is defined in terms of the number of signal events $N$ and the integrated luminosity $\mathcal{L}$ :

$$
\sigma=\frac{N}{\mathcal{L}}
$$

The cross-section may also be measured in terms of the binned distribution of a particular observable related to the process in question; this is known as the differential cross-section. For an observable $x$ distributed in $i$ bins of width $\Delta x_{i}$, the differential cross-section is:

$$
\frac{d \sigma_{i}}{d x_{i}}=\frac{N_{i}}{\mathcal{L} \Delta x_{i}}
$$


8. Measurement of the inclusive and differential fiducial cross-sections in the $H \rightarrow Z Z^{(*)} \rightarrow 4 \ell$ channel

Six observables of interest, which can provide valuable information for testing Standard Model predictions, have been chosen for this measurement. These observables are:

- Higgs boson transverse momentum $\left(\boldsymbol{p}_{\mathrm{T}, \boldsymbol{H}}\right)$ Measuring the reconstructed $p_{\mathrm{T}}$ distribution of the four-lepton system resulting from decays of Higgs bosons produced in proton-proton collisions can test the QCD predictions involved in the various production modes described in Section 2.5.2.

- Higgs boson rapidity $\left(\left|\boldsymbol{y}_{\boldsymbol{H}}\right|\right)$ The rapidity is defined as the boost along the beam axis (represented here by the $z$ axis):

$$
y=\frac{1}{2} \ln \frac{E+p_{z}}{E-p_{z}}
$$

This observable, estimated by reconstructing the four-lepton system produced via Higgs boson decays, can provide information about the parton distribution functions of the proton.

- Subleading $\boldsymbol{Z}$ boson mass $\left(\boldsymbol{m}_{\mathbf{3 4}}\right)$ The mass distribution of the subleading dilepton is influenced by the spin of the Higgs boson.

- Production angle of the leading $\boldsymbol{Z}$ boson $\left(\left|\cos \boldsymbol{\theta}^{*}\right|\right)$ Defined as the angle between the collision axis and the direction of the leading $Z$ boson (refer to Figure 7.12), this observable is sensitive to the Higgs boson's spin and parity.

- Jet multiplicity $\left(\boldsymbol{n}_{\text {jets }}\right)$ The number of jets associated with the Higgs boson is directly related to its production mechanisms. The dominant mechanism, gluon-gluon fusion (ggF), typically involves zero or one associated jet. The mechanisms of vector boson fusion and associated production with a vector 
8. Measurement of the inclusive and differential fiducial cross-sections in the $H \rightarrow Z Z^{(*)} \rightarrow 4 \ell$ channel

boson (VBF and VH, respectively), result in two jets. When the Higgs boson is produced in association with a $t \bar{t}$ pair $(t \bar{t} H$; the rarest mechanism at the LHC), three or more jets are typically observed as a result of the dominant hadronic $t \bar{t}$ decays.

- Leading jet transverse momentum $\left(\boldsymbol{p}_{\mathrm{T}}\right.$, jet $)$ Measuring this observable probes the various production modes and tests the modelling of the QCD processes involved.

The observables are separated into several bins for the differential crosssection measurements. Since the statistics are still limited for these first measurements, a small number of fairly wide bins (i.e. wider than the resolution and scale uncertainty) was chosen for each observable, in order to optimize the statistical significance and minimize the effect of migration between bins. The choice of bins was also synchronized with that of the $H \rightarrow \gamma \gamma$ analysis [119] in order to allow for future combinations of the respective measurements. Details of the binning are given in Table 8.1.

\begin{tabular}{lcc}
\hline \hline Observable & $N_{\text {bins }}$ & Bin limits \\
\hline$p_{\mathrm{T}, H}$ & 4 & $\{0,20,50,100,200\}[\mathrm{GeV}]$ \\
$\left|y_{H}\right|$ & 5 & $\{0,0.3,0.6,0.9,1.2,2.4\}$ \\
$m_{34}$ & 4 & $\{12,20,30,40,60\}[\mathrm{GeV}]$ \\
$\left|\cos \theta^{*}\right|$ & 5 & $\{0,0.2,0.4,0.6,0.8,1\}$ \\
$n_{\text {jets }}$ & 4 & $\{0,1,2, \geq 3\}$ \\
$p_{\mathrm{T}, \text { jet }}$ & 4 & $\{0,30,50,70,140\}[\mathrm{GeV}]$ \\
\hline \hline
\end{tabular}

TABLE 8.1: Binning for the six observables used for the differential cross-section measurement. 


\subsection{Definition of the fiducial region}

The cross-section measurement is performed within a fiducial volume in which the detector provides sufficient coverage to measure the signal. This eliminates modeldependent uncertainties related to extrapolating the measurement to regions not covered by the detector. The event selection corresponding to the fiducial region, summarized in Table 8.2, has been chosen to be as similar as possible to that of the main analysis described in Section 5.3. Differences between the fiducial and reconstruction-level selections are described below.

Jets must satisfy the kinematic requirements: $p_{\mathrm{T}}>30 \mathrm{GeV}$ and $|y|<4.4$. These requirements were chosen for the sake of compatibility with the $H \rightarrow \gamma \gamma$ analysis. Only the $8 \mathrm{TeV}$ data, corresponding to an integrated luminosity of $20.3 \mathrm{fb}^{-1}$, are considered in this particular analysis.

Since isolation cuts can be complicated (and model-dependent) to define at the truth level, the isolation and impact parameter significance cuts are not applied in the fiducial selection. Instead, leptons in the fiducial region are required to originate from vector boson decays. Events containing $\tau$-leptons in the final state are removed from the fiducial region.

An additional requirement is imposed on the invariant mass of candidate Higgs boson events; selected events must occur within the mass window $119 \mathrm{GeV}<$ $m_{4 \ell}<128 \mathrm{GeV}$. This window was chosen in order to maximize the statistical significance $S / \sqrt{S+B}$, where $S$ and $B$ are the measured signal and background yields, respectively.

The acceptance and efficiency of the fiducial selection have been studied using simulated $H \rightarrow Z Z^{(*)} \rightarrow 4 \ell$ samples (refer to Section 5.1) corresponding 
8. Measurement of the inclusive and differential fiducial cross-sections in the $H \rightarrow Z Z^{(*)} \rightarrow 4 \ell$ channel

\begin{tabular}{ll}
\hline \hline & \multicolumn{1}{c}{ Lepton selection } \\
\hline Muons & $p_{\mathrm{T}}>6 \mathrm{GeV},|\eta|<2.7$ \\
Electrons & $p_{\mathrm{T}}>7 \mathrm{GeV},|\eta|<2.47$ \\
\hline \multicolumn{1}{c}{ Lepton pairing } \\
\hline Leading pair & \multicolumn{1}{c}{ Same-flavour, opposite-sign pair with smallest $\left|m_{Z}-m_{\ell \ell}\right|$} \\
Subleading pair & Remaining same-flavour, opposite-sign pair with smallest $\left|m_{Z}-m_{\ell \ell}\right|$ \\
\hline & \multicolumn{1}{c}{ Event selection } \\
\hline Lepton kinematics & $p_{\mathrm{T}}>20,15,10 \mathrm{GeV}$ \\
Mass requirements & $50<m_{12}<106 \mathrm{GeV}$ \\
& $12<m_{34}<115 \mathrm{GeV}$ \\
Lepton separation & $\Delta R\left(\ell_{i}, \ell_{j}\right)>0.1(0.2)$ for same- (different-) flavour leptons \\
$J / \Psi$ veto & $m\left(\ell_{i}, \ell_{j}\right)>5 \mathrm{GeV}$ for all same-flavour, opposite-sign pairs \\
Mass window & $118<m_{4 \ell}<129 \mathrm{GeV}$ \\
\hline \hline
\end{tabular}

TABle 8.2: Definition of the fiducial region for the differential cross-section measurement.

to the production modes introduced in Section 2.5.2. The fiducial acceptance, defined as the ratio of the number of events in the fiducial region to the number of generated events, is $45.7 \%$. The fiducial efficiency is defined as the ratio of the number of reconstructed events, $N_{\text {reco }}$, to the true number of events in the fiducial region, $N_{\text {fid }}$. For a Standard Model Higgs boson with mass $m_{H}=125.4 \mathrm{GeV}$, $N_{\text {reco }} / N_{\text {fid }}=55.3 \%$. In total, 34 events satisfying the event selection are observed.

The theoretical predictions used for this analysis are calculated for a Standard Model Higgs boson of mass $m_{H}=125.4 \mathrm{GeV}$ (the result of the combined measurement using the $H \rightarrow Z Z^{(*)} \rightarrow 4 \ell$ and $H \rightarrow \gamma \gamma$ channels [4]), normalized to the most up-to-date predictions of the Standard Model cross-sections [120], and corrected for the fiducial acceptance.

\subsection{Bin-by-bin unfolding}

Since the detector cannot reconstruct $100 \%$ of the events in the fiducial region, and it cannot perfectly measure the kinematic properties of the events it does 
8. Measurement of the inclusive and differential fiducial cross-sections in the $H \rightarrow Z Z^{(*)} \rightarrow 4 \ell$ channel

reconstruct, the measured signal yield must be corrected to its true distribution in order to compensate for efficiency and resolution effects. This procedure is known as "unfolding." For the main analysis, this is done using bin-by-bin correction factors. An alternative method for unfolding is presented in Section 8.5.

\subsubsection{Correction factors}

In the case of a detector having a fiducial efficiency $\epsilon_{\text {fid }}$, the measured signal yield must be unfolded to its true value using a correction factor $C$, which is derived from simulation:

$$
C=\frac{1}{\epsilon_{\text {fid }}}=\frac{N_{\text {fid }}}{N_{\text {reco }}}
$$

Then, the inclusive fiducial cross-section defined in Equation 8.1 becomes:

$$
\sigma=\frac{N}{\epsilon_{\text {fid }} \cdot \mathcal{L}}=C \cdot \frac{N}{\mathcal{L}}
$$

For the differential cross-section measurement, the fiducial efficiencies are determined in the bins of the observable, and so binned correction factors $C_{i}$ are used:

$$
\begin{gathered}
C_{i}=\frac{1}{\epsilon_{\mathrm{fid}, i}}=\frac{N_{\mathrm{fid}, i}}{N_{\mathrm{reco}, i}} \\
\frac{d \sigma_{i}}{d x_{i}}=C_{i} \cdot \frac{N_{i}}{\mathcal{L} \Delta x_{i}} .
\end{gathered}
$$

The correction factors are derived using samples of simulated $H \rightarrow Z Z^{(*)} \rightarrow$ $4 \ell$ decays with $m_{H}=125 \mathrm{GeV}$, including QED corrections for final-state radiation (FSR) emission. Since photon emission alters the curvature of the lepton's trajectory, its final reconstructed $p_{\mathrm{T}}$ may be underestimated if only the contribution of 
8. Measurement of the inclusive and differential fiducial cross-sections in the $H \rightarrow Z Z^{(*)} \rightarrow 4 \ell$ channel

the "bare" final-state lepton is considered; this effect should be corrected in order to ensure accurate unfolding.

It is possible to "dress" the leptons by adding the four-momentum of nearby (in $\Delta R$-space) photons, as shown in Figure 8.1 in order to compensate for momentum losses; this technique works well for electrons since it closely replicates what is actually measured in the calorimeter. However, this technique tends to overestimate muons' $p_{\mathrm{T}}$, compared to the reconstructed observable, since the muon $p_{\mathrm{T}}$ measurement is based on tracking information.

Another technique exploits the information about the intermediate-state leptons provided by the simulations. It is possible to consider instead a lepton's "Born-level" momentum; i.e. its momentum before radiation is emitted. This provides a good representation of the lepton's true momentum, and works well for both electrons and muons. For this analysis, it was chosen to perform the fiducial selection to derive the true number of events, $N_{\text {fid, } i}$ at the Born level.

As shown in Table 8.3 for the example of the inclusive case, the correction factors are dependent on the production mode. The correction factors for the six observables of interest, as well as the inclusive correction factor, are given in Table 8.4. All of the Higgs boson production modes at the LHC (ggF, VBF, WH, $\mathrm{ZH}$, and $t \bar{t} \mathrm{H}$ ), weighted by their respective contributions to the total number of events, are used to derive these correction factors. Since the observed event yield is dominated by the ggF mode, the inclusive correction factor is nearly identical to that given in Table 8.3 for the ggF mode. 


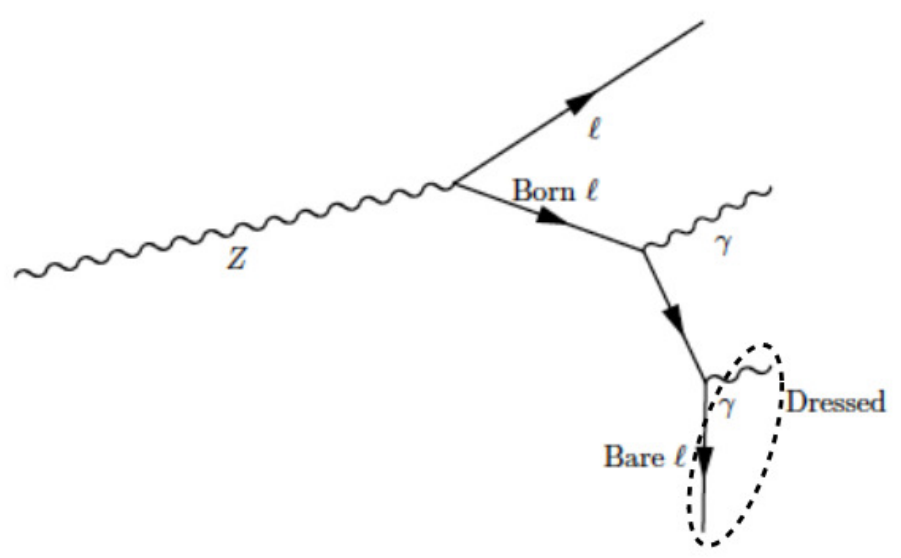

FiguRE 8.1: Schematic of truth-level lepton kinematics and photon emission. The final-state lepton on its own is considered to be "bare." This lepton may be "dressed" by adding the $p_{\mathrm{T}}$ contribution of nearby photons (enclosed by the dashed line) in order to better represent its true $p_{\mathrm{T}}$. At the Born level, the intermediate-state lepton (i.e. before radiation is emitted) is considered.

\begin{tabular}{lc}
\hline \hline Mode & Inclusive correction factor \\
\hline ggF & $1.796 \pm 0.009$ \\
$\mathrm{VBF}$ & $1.737 \pm 0.008$ \\
$\mathrm{WH}$ & $1.849 \pm 0.014$ \\
$\mathrm{ZH}$ & $1.800 \pm 0.015$ \\
$t \bar{t} \mathrm{H}$ & $2.371 \pm 0.022$ \\
\hline \hline
\end{tabular}

TABLE 8.3: Inclusive correction factors, calculated at the Born level, as defined in Equation 8.4 for each Higgs boson production mode. Uncertainties are statistical only.

\subsubsection{Study of the model-dependence of the correction fac- tors}

The correction factors are derived using a combination of simulated samples corresponding to each the Higgs boson production modes, weighted according to the predicted Standard Model cross-section for each mode listed in Table 5.1. Since 
8. Measurement of the inclusive and differential fiducial cross-sections in the $H \rightarrow Z Z^{(*)} \rightarrow 4 \ell$ channel

\begin{tabular}{|c|c|c|c|c|c|}
\hline \multirow[t]{2}{*}{ Observable } & \multicolumn{5}{|c|}{ Correction factor } \\
\hline & Bin 1 & Bin 2 & Bin 3 & $\operatorname{Bin} 4$ & $\operatorname{Bin} 5$ \\
\hline Inclusive & $1.796 \pm 0.008$ & & & & \\
\hline$p_{\mathrm{T}, H}$ & $1.781 \pm 0.015$ & $1.801 \pm 0.013$ & $1.821 \pm 0.015$ & $1.780 \pm 0.020$ & \\
\hline$\left|y_{H}\right|$ & $1.770 \pm 0.017$ & $1.733 \pm 0.016$ & $1.767 \pm 0.018$ & $1.807 \pm 0.020$ & $1.888 \pm 0.017$ \\
\hline$m_{34}$ & $1.743 \pm 0.018$ & $1.758 \pm 0.011$ & $1.912 \pm 0.018$ & $1.776 \pm 0.023$ & \\
\hline$\left|\cos \theta^{*}\right|$ & $1.786 \pm 0.017$ & $1.766 \pm 0.017$ & $1.806 \pm 0.018$ & $1.803 \pm 0.018$ & $1.822 \pm 0.019$ \\
\hline$n_{\text {jets }}$ & $1.895 \pm 0.012$ & $1.741 \pm 0.013$ & $1.596 \pm 0.016$ & $1.470 \pm 0.020$ & \\
\hline$p_{\mathrm{T}, \text { jet }}$ & $1.895 \pm 0.012$ & $1.661 \pm 0.015$ & $1.683 \pm 0.019$ & $1.675 \pm 0.016$ & \\
\hline
\end{tabular}

TABLE 8.4: Correction factors, calculated at the Born level, as defined in Equation 8.4 and Equation 8.6 for the inclusive and differential cross-section measurements, respectively. Uncertainties are statistical only.

the cross-sections have not been measured experimentally, it is possible that the use of the theoretical values could bias the results.

In order to test the model-dependence of the correction factors, several simulated ggF samples corresponding to the possible spin/parity configurations $\left(0^{+}, 0^{-}, 2^{+}\right)$were used to derive correction factors for the six observables. For the Standard Model $0^{+}$hypothesis, two generators were compared: PowHEG, the nominal, NNLO generator; and JHU [118, 121], a LO generator. JHU was used for the $0^{-}$and $2^{+}$hypotheses.

The correction factors for each sample were compared to those calculated using the $\mathrm{JHU} 0^{+}$sample; this was chosen as the reference sample for the purposes of this study in order to disentangle the effects due to the generator from those due to the spin/parity. The relative difference between the correction factors for each sample and the reference sample are shown in Figure 8.2. In general, the variation is small - on the order of $5 \%$ or less. This variation does not contribute to the systematic uncertainty of the method of correction factors, but is used to illustrate the model-independence of the method. 
8. Measurement of the inclusive and differential fiducial cross-sections in the $H \rightarrow Z Z^{(*)} \rightarrow 4 \ell$ channel

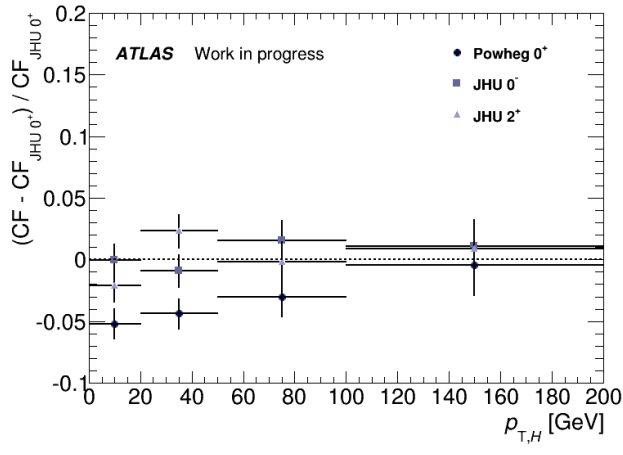

(a) $p_{\mathrm{T}, H}$

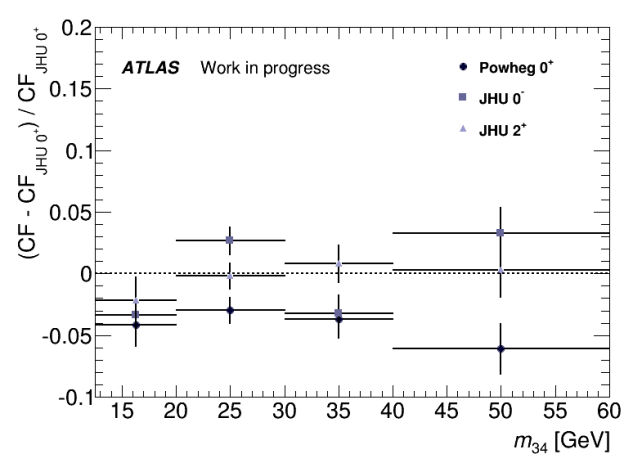

(c) $m_{34}$

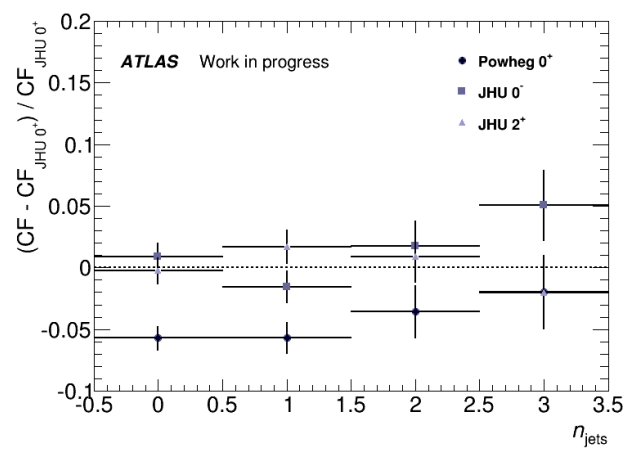

(e) $n_{\text {jets }}$

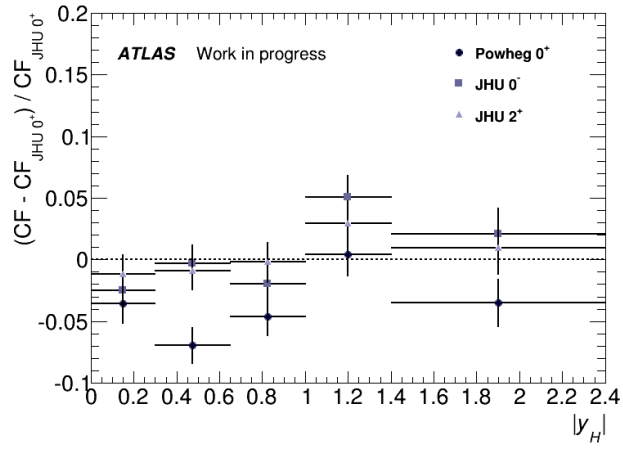

(b) $\left|y_{H}\right|$

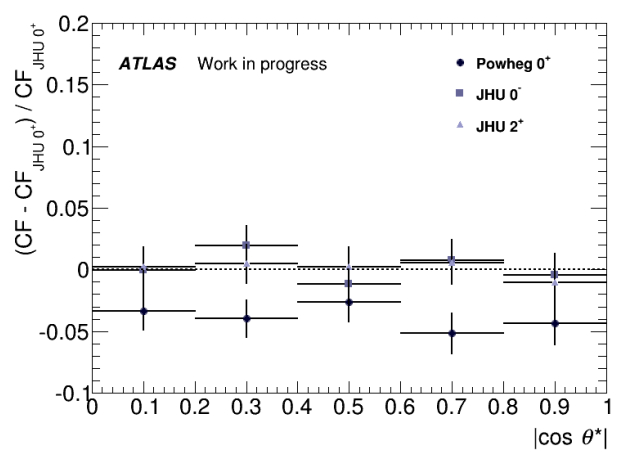

(d) $\left|\cos \theta^{*}\right|$

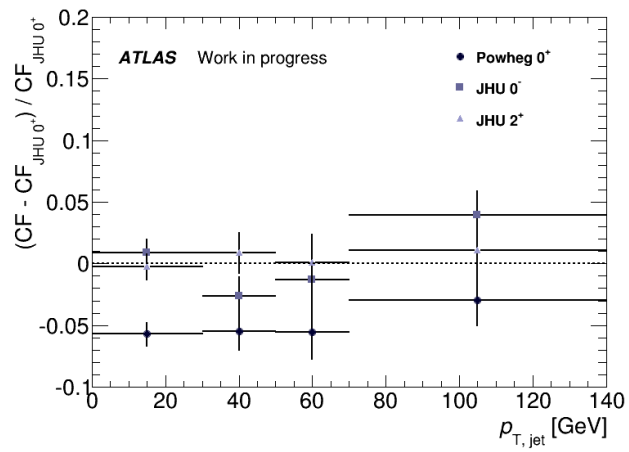

(f) $p_{\mathrm{T}}$, jet

Figure 8.2: Comparison of correction factors (CF), defined by Equation 8.6, for various spin/parity hypotheses, compared to the Standard Model $J^{P}=0^{+}$ hypothesis derived using the JHU generator. 


\subsubsection{Systematic uncertainties}

Theoretical and experimental systematic uncertainties are estimated for the backgrounds and for the correction factors. These uncertainties affect two aspects of the measurement; first, uncertainties on the background affect the measure number of signal events, while uncertainties on the correction factors affect the final extraction of the cross-section.

For the backgrounds, the main experimental sources of systematic uncertainty include the luminosity, which is used to estimate the $Z Z$ background from simulation, as well as the systematic uncertainties on the lepton trigger, reconstruction, and identification efficiencies described in Section 5.3 (these uncertainties are provided by the ATLAS combined performance working groups). The uncertainty on the data-driven reducible background estimates is estimated by varying the shapes and normalizations of the distributions. The variations were derived separately for the subleading dielectron and dimuon channels. For the subleading dielectron channels, data-driven methods were used to derive the estimates from two alternative control regions, one of which was enriched in heavy quark decays (known as the "upper" variation), while the other, known as the "lower" variation, featured relaxed electron identification, isolation, and impact parameter significance cuts. The subleading dimuon backgrounds were estimated from simulation, by tightening the isolation and impact parameter significance requirements for the upper variation and loosening them for the lower variation. These shape variations are shown in Figure 8.3

Theoretical sources of systematic uncertainty on the backgrounds include the uncertainties on the parton distribution functions (PDFs) and QCD scale, which are evaluated as described in Section 7.3.1. 


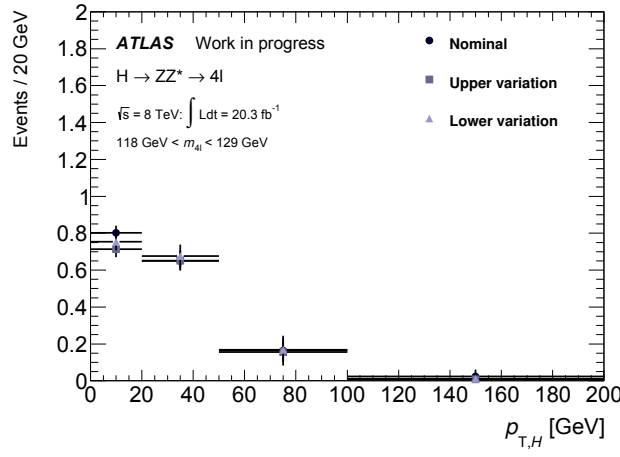

(a) $p_{\mathrm{T}, H}$

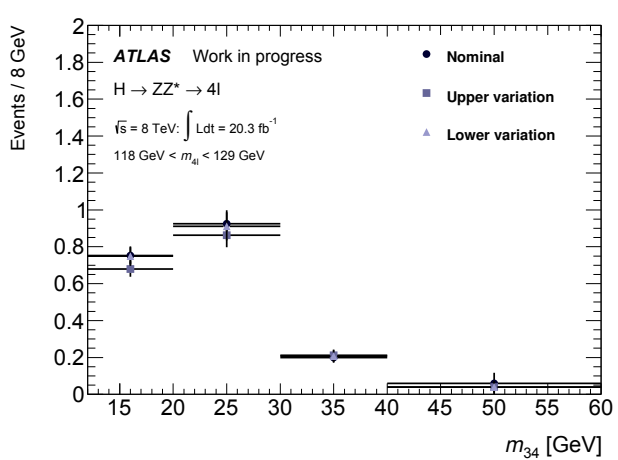

(c) $m_{34}$

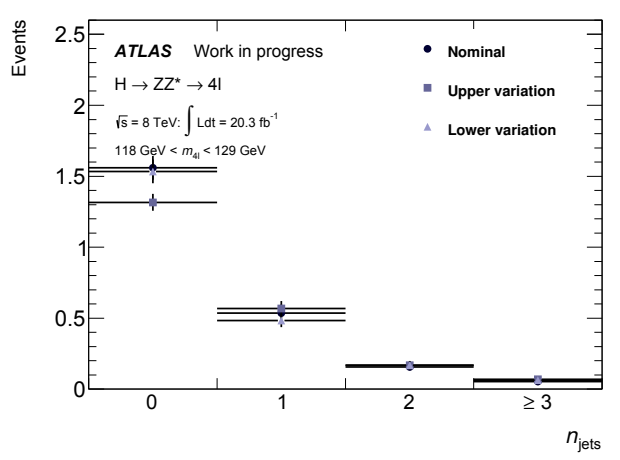

(e) $n_{\text {jets }}$

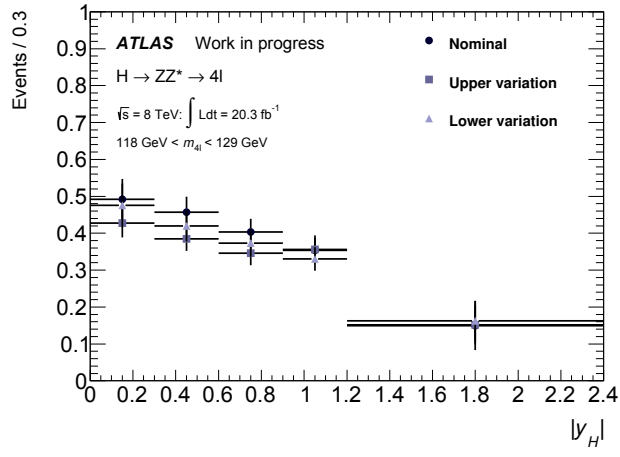

(b) $\left|y_{H}\right|$

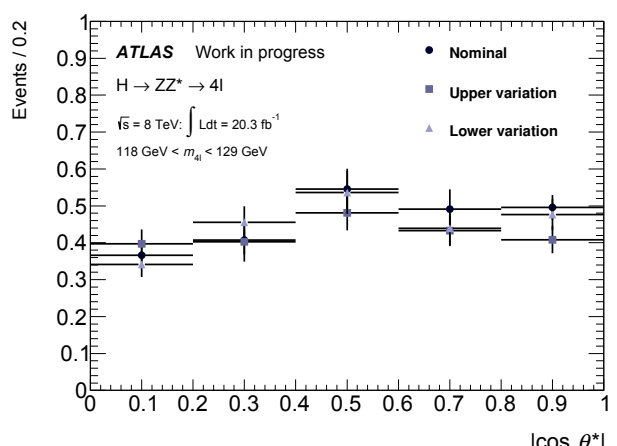

(d) $\left|\cos \theta^{*}\right|$

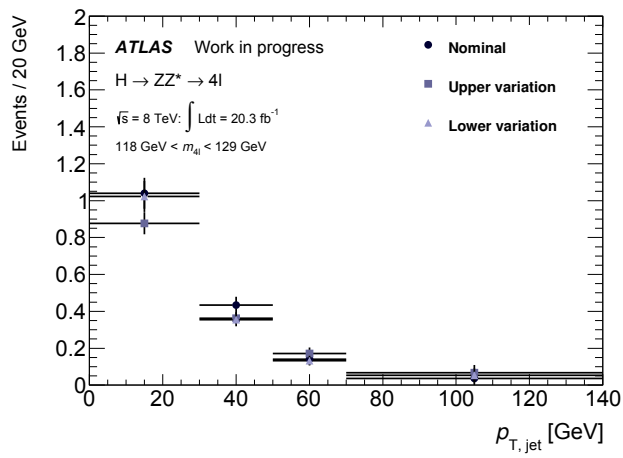

(f) $p_{\mathrm{T}, \text { jet }}$

FiguRE 8.3: Reducible background shape variations used to determine the systematic uncertainty due to the background estimation. The nominal reducible background shape is also shown for comparison. 
8. Measurement of the inclusive and differential fiducial cross-sections in the $H \rightarrow Z Z^{(*)} \rightarrow 4 \ell$ channel

Several of these factors, including the luminosity and the lepton efficiencies, also affect the calculation of the correction factors. Also considered are differences between simulation and data in the jet resolution, energy scale, and flavour composition $[110,122,123]$. The correction factors are dependent on the Higgs boson's mass, and so the effects of the experimental uncertainty on this quantity is evaluated by checking the mass-dependence of the correction factors.

The effects on the correction factors due to theoretical uncertainties on the predicted cross-sections for the Higgs boson's production modes are determined by varying the predictions within the current experimental limits [6].

A summary of the dominant sources of systematic uncertainty is given in Table 8.5.

\begin{tabular}{lc}
\hline \hline \multicolumn{2}{c}{ Systematic uncertainty (\%) } \\
\hline \multicolumn{2}{c}{ Background } \\
\hline Luminosity & $1.4-2.3$ \\
Reducible background & $1.6-34$ \\
Experimental, leptons & $1.3-2.3$ \\
PDF / scale & $3.0-24$ \\
\hline \multicolumn{2}{c}{ Correction factors } \\
\hline Luminosity & 2.8 \\
Experimental, leptons & $2.1-2.6$ \\
Experimental, jets & $2.7-13$ \\
Higgs boson mass & $0.4-2.7$ \\
Production mode & $0.1-15$ \\
\hline \hline
\end{tabular}

TABLE 8.5: Summary of the systematic uncertainties on the background estimation and correction factors contributing to the differential cross-section measurement. 


\subsection{Results}

\subsubsection{Inclusive fiducial cross-section}

The signal yield is extracted by performing a fit to the $m_{4 \ell}$ distribution using signal and background shape templates, with the mass of the Higgs boson fixed to $125.4 \mathrm{GeV}$, which is the result of the combined mass measurement from the $H \rightarrow Z Z^{(*)} \rightarrow 4 \ell$ and $H \rightarrow \gamma \gamma$ channels [4]. The number of signal events after subtracting the background is determined to be $23.7_{-5.3}^{+5.9}$ (stat.) ${ }_{-0.6}^{+0.6}$ (syst.), for $20.3 \mathrm{fb}^{-1}$ of data. The fiducial cross-section is determined by applying the inclusive correction factor given in Table 8.4 using Equation 8.5:

$$
\sigma_{\text {fid }}=2.11_{-0.47}^{+0.53} \text { (stat.) } \pm 0.8 \text { (syst.)fb. }
$$

The theoretical prediction, calculated as described in Section 8.2 for a Standard Model Higgs boson of mass $m_{H}=125.4 \mathrm{GeV}$ is $1.30 \pm 0.13 \mathrm{fb}[120]$.

\subsubsection{Differential fiducial cross-sections}

Since the number of events reconstructed in the fiducial region (34) is small, the signal yield in each observable bin is extracted by subtracting the predicted backgrounds derived using the methods described in Section 5.4 from the observed number of events in the data. This method assumes that the bin widths are sufficiently large that the migration of events between bins is minimal; this is a safe assumption in the case of a small number of wide bins as in this analysis. The resulting number of signal events is $25.1_{-5.4}^{+6.3}$ (stat. $)_{-0.4}^{+0.6}$ (syst.). This number differs from the yield used for the inclusive measurement in Section 8.4.1, since the 
8. Measurement of the inclusive and differential fiducial cross-sections in the $H \rightarrow Z Z^{(*)} \rightarrow 4 \ell$ channel

latter quantity was extracted by fixing the Higgs boson's mass to $125.4 \mathrm{GeV}$. The observed yields are shown in Figure 8.4 for the six observables of interest.

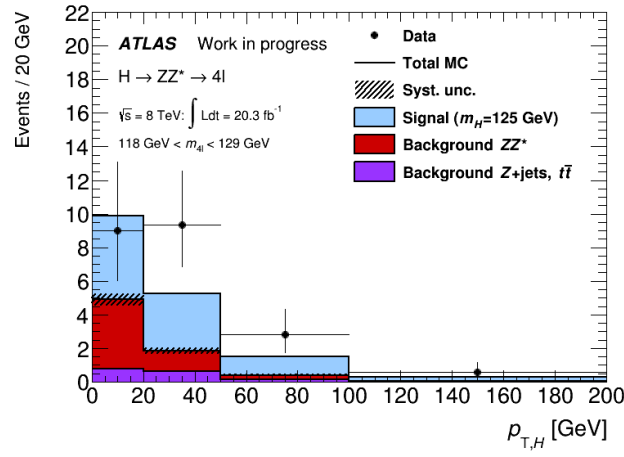

(a) $p_{\mathrm{T}, H}$

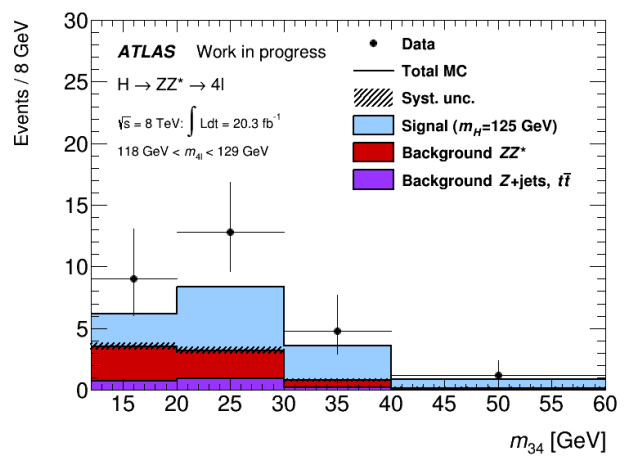

(c) $m_{34}$

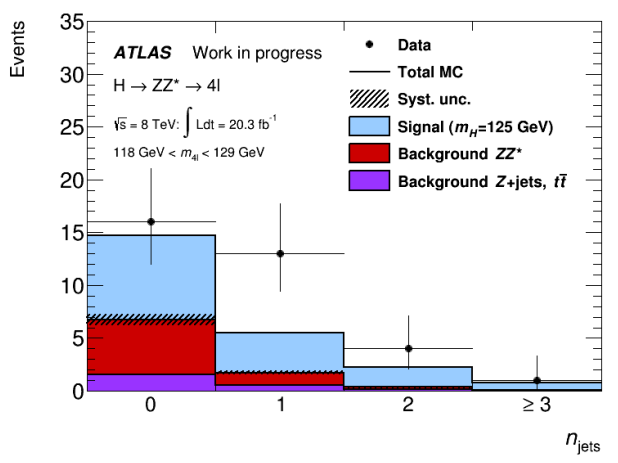

(e) $n_{\text {jets }}$

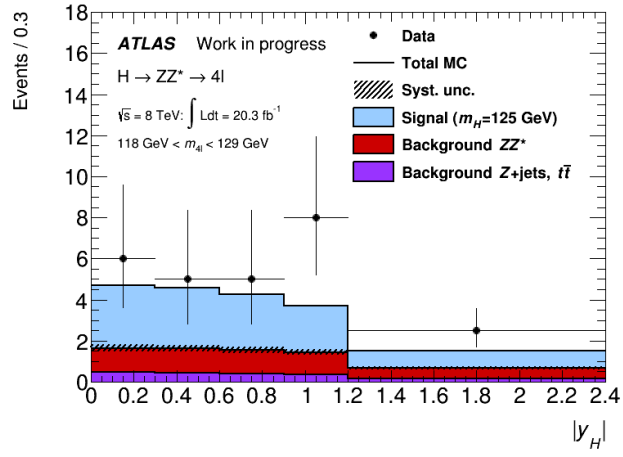

(b) $\left|y_{H}\right|$

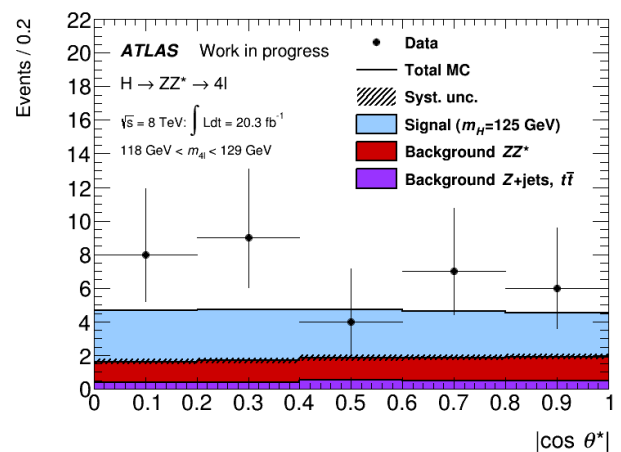

(d) $\left|\cos \theta^{*}\right|$

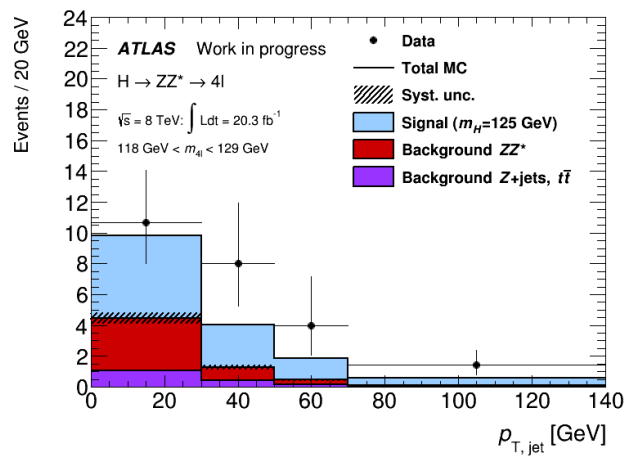

(f) $p_{\mathrm{T}, \text { jet }}$

Figure 8.4: Observed yields for the six observables used for the differential cross-section measurements with the $8 \mathrm{TeV}$ data, as well as the predicted reducible and irreducible background yields. Note that the distributions have variable bin widths, and so they are normalized to the bin width specified on the vertical axis. 
8. Measurement of the inclusive and differential fiducial cross-sections in the $H \rightarrow Z Z^{(*)} \rightarrow 4 \ell$ channel

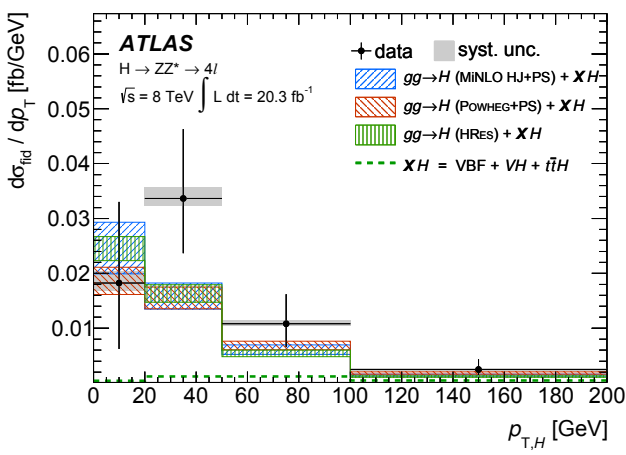

(a) $p_{\mathrm{T}, H}$

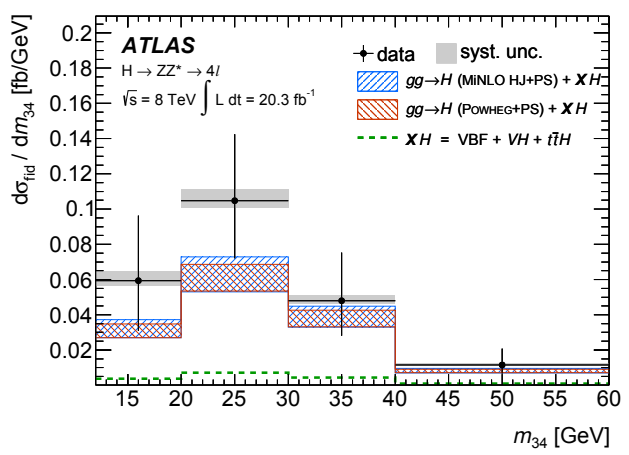

(c) $m_{34}$

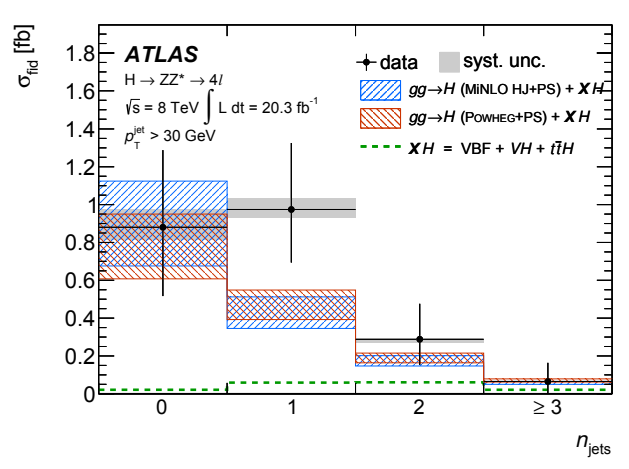

(e) $n_{\text {jets }}$

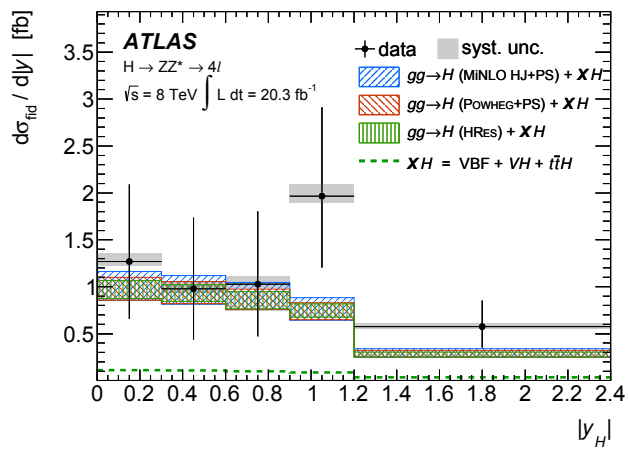

(b) $\left|y_{H}\right|$

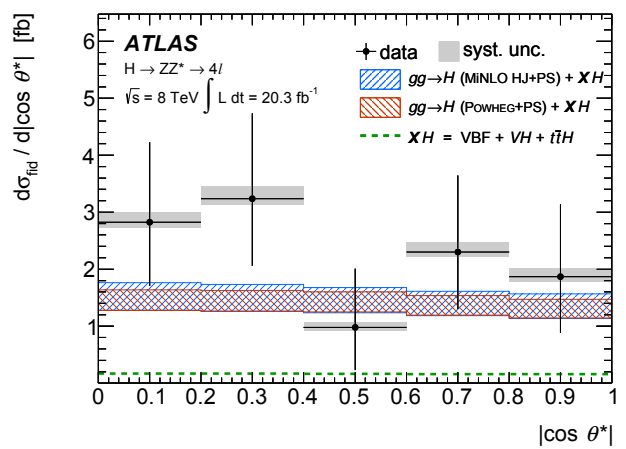

(d) $\left|\cos \theta^{*}\right|$

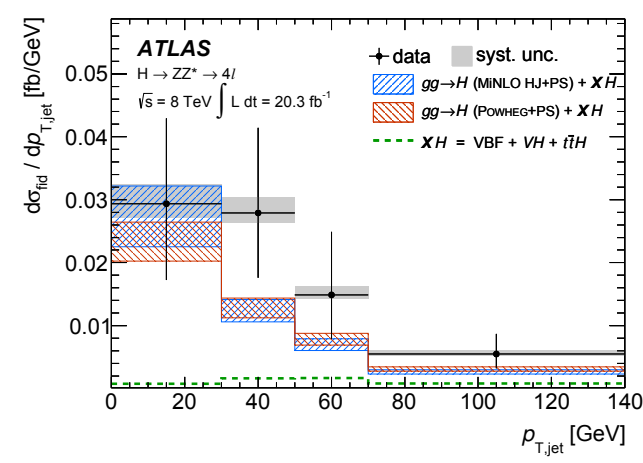

(f) $p_{\mathrm{T}, \text { jet }}$

Figure 8.5: Differential cross-sections for the Higgs boson in the $H \rightarrow Z Z^{(*)} \rightarrow$ $4 \ell$ channel. Several theoretical predictions are shown for comparison. [7] 
8. Measurement of the inclusive and differential fiducial cross-sections in the $H \rightarrow Z Z^{(*)} \rightarrow 4 \ell$ channel

To determine the differential cross-section for each of the observables, the correction factors given in Table 8.4 are used to unfold the observed signal to the true distribution using Equation 8.7. The resulting distributions are shown in Figure 8.5. The results are compared to three theoretical calculations: PowHEG, Minlo (NLO QCD) [124], and HRES2 (which models only the kinematic properties of the Higgs boson) [125, 126].

The results are compared to the predicted values using the profile likelihood ratio $\Lambda[127]$ :

$$
\Lambda=\frac{L\left(s_{i}, \hat{\hat{\theta}_{j}}\right)}{L\left(\hat{s}_{i}, \hat{\theta}_{j}\right)},
$$

where $s_{i}$ is the number of signal events in a given bin and the $\theta_{j}$ are nuisance parameters corresponding to the systematic uncertainties. In the numerator, $\hat{\theta}_{j}$ represents the value of $\theta$ that maximizes the likelihood $L$ for a given value of $s_{i}$. In the denominator, $\hat{s}_{i}$ and $\hat{\theta}_{j}$ are the unconditional maximum likelihood estimators. The likelihood function, $L$, is the product of Poisson pdfs corresponding to the signal for each bin and Gaussian-distributed nuisance parameters corresponding to the systematic uncertainties.

A test statistic can then be constructed by minimizing the negative logarithm of the profile likelihood ratio:

$$
t=-2 \ln \Lambda
$$

This quantity, which behaves as a $\chi^{2}$ distribution with the number of degrees of freedom equal to the number of bins for a given observable, is compared at the best-fit value for the number of signal events and at the value obtained with the cross-section fixed to the theoretically predicted value. The p-values corresponding 
8. Measurement of the inclusive and differential fiducial cross-sections in the $H \rightarrow Z Z^{(*)} \rightarrow 4 \ell$ channel

to these comparisons are shown in Table 8.6. No significant deviations from the Standard Model predictions are observed.

\begin{tabular}{lccc}
\hline \hline Observable & \multicolumn{3}{c}{ p-value } \\
& PowHEG & MinlO & HRES2 \\
\hline$p_{\mathrm{T}, H}$ & 0.30 & 0.23 & 0.16 \\
$\left|y_{H}\right|$ & 0.37 & 0.45 & 0.36 \\
$m_{34}$ & 0.48 & 0.60 & - \\
$\left|\cos \theta^{*}\right|$ & 0.35 & 0.45 & - \\
$n_{\text {jets }}$ & 0.37 & 0.28 & - \\
$p_{\mathrm{T}, \text { jet }}$ & 0.33 & 0.26 & - \\
\hline \hline
\end{tabular}

TABLE 8.6: The p-values for the comparison of the measured differential crosssections with three theoretical calculations. The p-values are computed from the difference of the negative logarithm of the profile likelihood ratio described by Equation 8.9 at the best fit value and with the cross-sections fixed to the theoretical predictions.

\subsection{The Bayesian iterative unfolding method}

The bin-by-bin unfolding method described in Section 8.4 .2 can be biased due to the effects of bin migration; i.e. the possibility that an event generated in a particular observable bin could be reconstructed in another bin due to energy and momentum resolution or scale uncertainties. In order to avoid this, it is necessary to ensure that the bin width is larger than the detector resolution in order to mitigate bin migration. In the low-statistics regime, as in the case of this analysis, where the data are binned in a small number of wide bins, it is generally safe to assume that bin migration is minimal. A study has been performed to investigate the use of an alternative unfolding method that takes into account the effects of bin migration using an iterative technique. 


\subsubsection{Application of Bayes' theorem to the unfolding prob- lem}

The Bayesian iterative unfolding method [128] was designed to evaluate the probability that a particular cause $C_{i}$ produces a particular effect $E_{j}$, e.g. that an event with the true value of a given observable occurring in bin $i$ might be measured in bin $j$. This can be described using Bayes' theorem, which states that the posterior probability $P\left(C_{i} \mid E_{j}\right)$ (i.e. the probability that cause $i$ occurred, given that effect $j$ was measured) is given by the expression:

$$
P\left(C_{i} \mid E_{j}\right)=\frac{P\left(E_{j} \mid C_{i}\right) P_{0}\left(C_{i}\right)}{\sum_{k=1}^{n_{C}} P\left(E_{j} \mid C_{k}\right) P_{0}\left(C_{k}\right)},
$$

where $P_{0}\left(C_{i}\right)$ is the prior probability distribution of the cause and $P\left(E_{j} \mid C_{i}\right)$ represents the response matrix, which gives the probability of observing an event in bin $j$, given that it was generated in bin $i$. The sum over the set of $n_{C}$ causes in the denominator is the probability of observing an event in bin $j, P\left(E_{j}\right)$.

Using this result, the expected number of causes in bin $i$ (i.e. the unfolded number of events) can then be estimated:

$$
\hat{n}\left(C_{i}\right)=\frac{1}{\epsilon_{i}} \sum_{j=1}^{n_{E}} P\left(C_{i} \mid E_{j}\right) n\left(E_{j}\right),
$$

where $n_{E}$ is the total number of measured events, $n\left(E_{j}\right)$ is the measured number of events in bin $j$ and $\epsilon_{i}=\sum_{j=1}^{n_{E}} P\left(E_{j} \mid C_{i}\right)$ is the efficiency of observing cause $C_{i}$ in any of the effects. 
8. Measurement of the inclusive and differential fiducial cross-sections in the $H \rightarrow Z Z^{(*)} \rightarrow 4 \ell$ channel

Given a distribution of observed events, $\boldsymbol{n}(E)$, the final probability that an event was generated in bin $i$ is:

$$
\hat{P}\left(C_{i}\right)=P\left(C_{i} \mid \boldsymbol{n}(E)\right)=\frac{\hat{n}\left(C_{i}\right)}{\hat{N}_{\text {true }}},
$$

where the estimated true total number of events $\hat{N}_{\text {true }}=\sum_{i} \hat{n}\left(C_{i}\right)$.

Then, the unfolding strategy is as follows: given the initial true distribution of an observable, $\boldsymbol{P}_{0}(C)$, and an observed number of events, $N_{\text {obs }}$, the expected number of unfolded events in bin $i$ is $n_{0}\left(C_{i}\right)=P_{0}\left(C_{i}\right) N_{\text {obs }}$. This can then be inserted into Equation 8.11, along with the response matrix $P\left(E_{j} \mid C_{i}\right)$ for that observable (in practice, this is determined using simulations). The resulting posterior probability $P\left(C_{i} \mid E_{j}\right)$, along with the measured distribution of events $\boldsymbol{n}(E)$, can then be used in Equation 8.12 to determine the unfolded number of events $\hat{n}\left(C_{i}\right)$. Using Equation 8.13, $\hat{P}\left(C_{i}\right)$ can be calculated and the result inserted back into Equation 8.11. This iterative procedure is then continued until the unfolded number of events converges.

\subsubsection{Bayesian iterative unfolding in the RooUnfold frame- work}

The Bayesian iterative unfolding method is implemented in the RooUnFoLD framework [129, 130], which relies on the ROOT [131] histogram classes. The method takes as input a histogram of the measured signal yield, as well as a response matrix derived from simulation. The number of iterations is specified by the user. In order to evaluate the statistical uncertainty on the unfolding, the 
8. Measurement of the inclusive and differential fiducial cross-sections in the $H \rightarrow Z Z^{(*)} \rightarrow 4 \ell$ channel

covariance matrix is computed using a set of toy Monte Carlo tests, in which the parameters of the simulated true distribution are varied slightly.

The response matrices are derived using a sample of simulated $H \rightarrow Z Z^{(*)} \rightarrow$ $4 \ell$ decays with $m_{H}=125 \mathrm{GeV}$; the rows correspond to the true observable values, while the columns correspond to the reconstructed values. In practice, the response matrix can be represented as a two-dimensional histogram; for each simulated event, the two-dimensional bin corresponding to that event's reconstructed and true observable values is filled (events failing the reconstruction selection are omitted). Each row is then normalized to the number of events in the truth bin corresponding to that row. Thus, the response matrix elements $R_{i j}$ correspond to the probability of an observable having a value in a given truth bin $j$ to end up being measured in a particular bin $i[132]$ :

$$
R_{i j}=P(\text { Measured in bin } i \mid \text { True value in bin } j) .
$$

Figure 8.6 shows the response matrices for the six observables of interest. It can be seen that, for the variables corresponding to the kinematics of the Higgs boson, migration between bins is, indeed, minimal since the matrices are essentially diagonal. For the jet variables, however, the bin migration is more significant.

Unfolded differential cross-sections have been determined using the Bayesian iterative unfolding method with $n=1, \ldots, 10$ iterations. The relative difference between the results of this method and the results of the bin-by-bin method are shown in Figure 8.7. Firstly, it is important to note that for all of the observables, the difference between the results of the Bayesian method and the bin-by-bin method is much smaller than the statistical uncertainty on the latter results (illustrated in Figure 8.7 by a shaded band). 


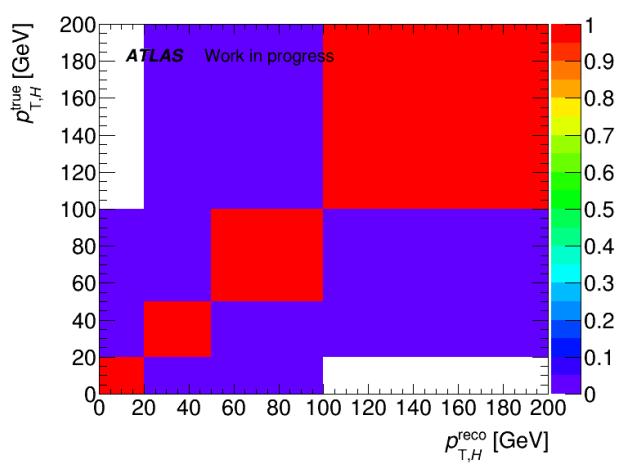

(a) $p_{\mathrm{T}, H}$

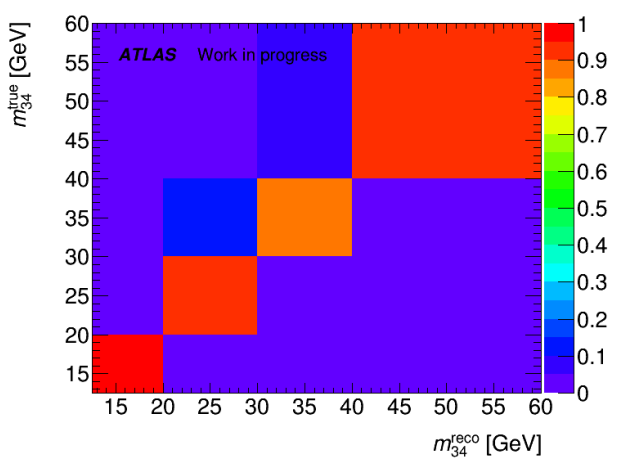

(c) $m_{34}$

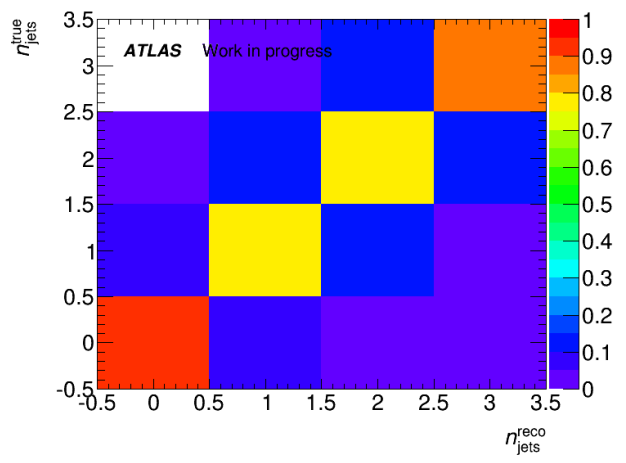

(e) $n_{\text {jets }}$

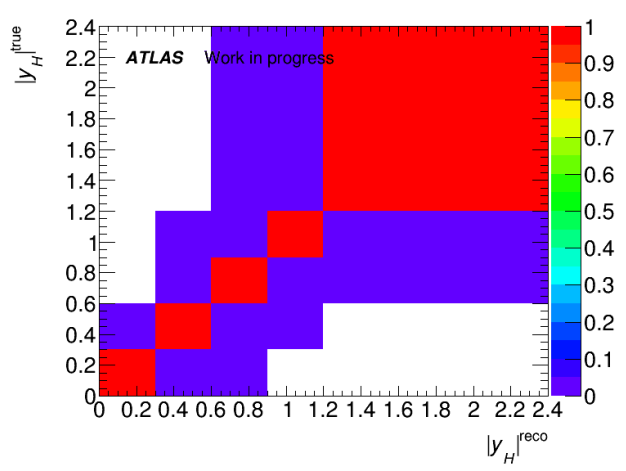

(b) $\left|y_{H}\right|$

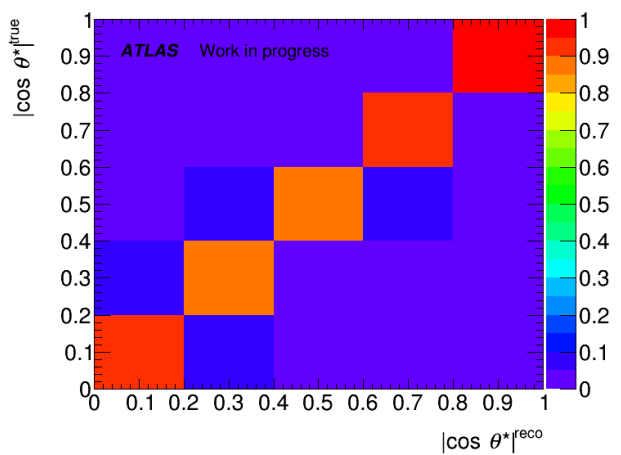

(d) $\left|\cos \theta^{*}\right|$

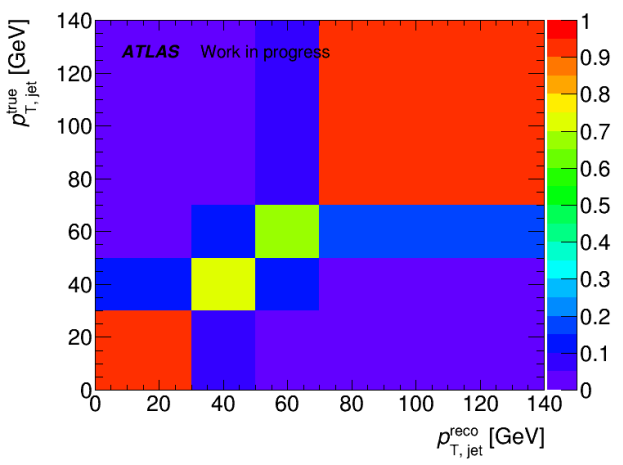

(f) $p_{\mathrm{T}, \text { jet }}$

FiguRE 8.6: Response matrices for the six observables of interest. The matrices are derived using simulated $H \rightarrow Z Z^{(*)} \rightarrow 4 \ell$ decays with $m_{H}=125 \mathrm{GeV}$; the rows correspond to the true observable values, while the columns correspond to the reconstructed values. The response matrix element $R_{i j}$ corresponds to the probability of an observable generated in a given bin $j$ to end up being measured in a particular bin $i$. This probability (in \%) is represented by the colour scheme. 


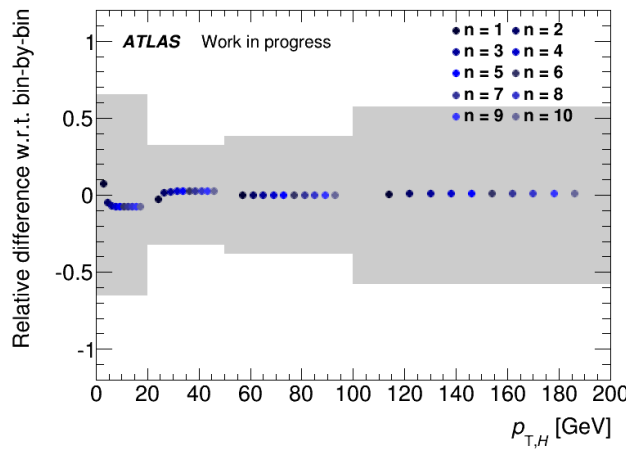

(a) $p_{\mathrm{T}, H}$

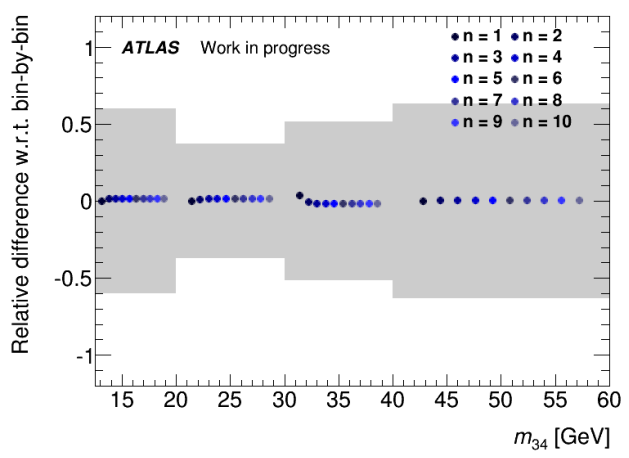

(c) $m_{34}$

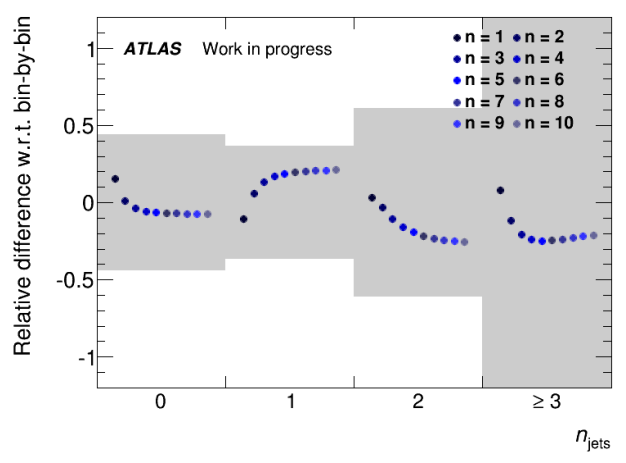

(e) $n_{\text {jets }}$

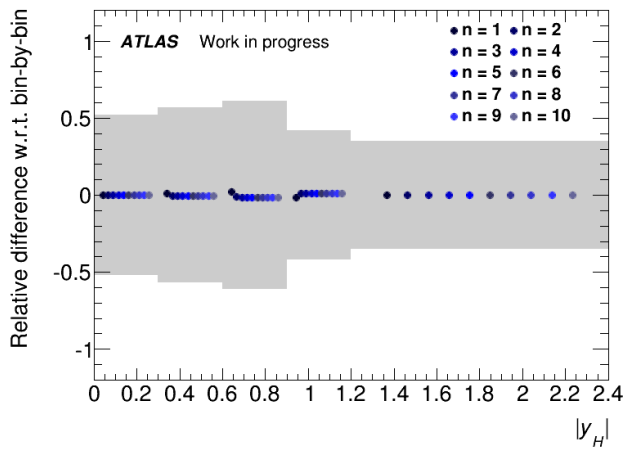

(b) $\left|y_{H}\right|$

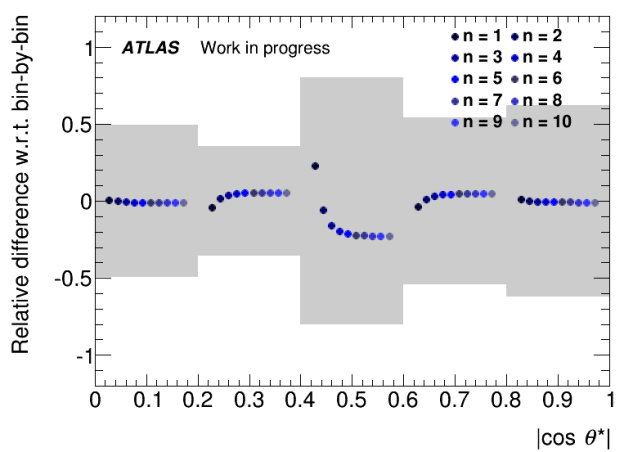

(d) $\left|\cos \theta^{*}\right|$

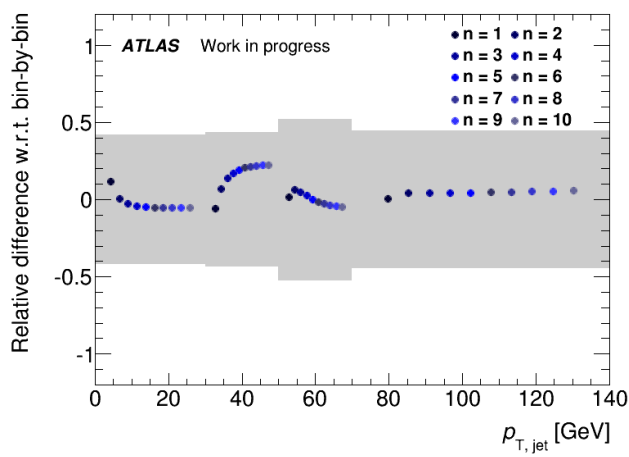

(f) $p_{\mathrm{T}, \text { jet }}$

FiguRE 8.7: Comparison of unfolded differential cross-sections determined using the Bayesian unfolding method with $n=1, \ldots, 10$ iterations. The points are slightly offset for clarity. The results are expressed in terms of the relative difference with respect to the results of the bin-by-bin unfolding method. Also shown for comparison are the statistical uncertainties on the bin-by-bin results (shaded bands). 
8. Measurement of the inclusive and differential fiducial cross-sections in the $H \rightarrow Z Z^{(*)} \rightarrow 4 \ell$ channel

It can be seen that, in general, the Bayesian method converges quickly, with fewer than 10 iterations required for all of the observables. Those observables (e.g. $\left.p_{\mathrm{T}, H},\left|y_{H}\right|\right)$ having the least bin migration (i.e. the most diagonal response matrices; cf. Figure 8.6) require the fewest iterations to converge. It is also interesting to note that for the variables with more bin migration $\left(\left|\cos \theta^{*}\right|, n_{\text {jets }}\right.$, and $p_{\mathrm{T}}$, jet $)$, the Bayesian method sometimes converges to a value somewhat far from the nominal method (although still within the statistical uncertainty). This suggests that the bin migration may have some effect on the calculation of the correction factors.

In order to determine a sufficient threshold for convergence, after each iteration, the algorithm compares the unfolded distribution $\boldsymbol{P}(C)$ with the prior distribution $\boldsymbol{P}_{0}(C)$ and calculates the $\chi^{2}$ value. This is typically large for the first few iterations, but quickly decreases to small values of order $10^{-4}$ or smaller as the unfolded result converges. For the final results, the number of iterations chosen was based on achieving a $\chi^{2}$ value of order $10^{-4}$ or smaller; the required number of iterations was found to be $n=4$ for $p_{\mathrm{T}, H},\left|y_{H}\right|$, and $m_{34} ; n=6$ for $\left|\cos \theta^{*}\right|$; and $n=9$ for $n_{\text {jets }}$ and $p_{\mathrm{T}, \text { jet }}$.

\subsubsection{Systematic uncertainties}

\subsubsection{Effect of the reducible background shapes}

Since the signal yield for the differential cross-section measurement is obtained by simply subtracting the background from the data (rather than performing a fit to the invariant mass distribution as was done for the inclusive measurement), it is important to understand the effect of the shape of the background on the result. 
8. Measurement of the inclusive and differential fiducial cross-sections in the $H \rightarrow Z Z^{(*)} \rightarrow 4 \ell$ channel

To quantify this effect, the measurements were repeated using four alternative reducible background shapes and normalizations:

- The nominal background shape was kept, but scaled up by a factor of (1+ the statistical uncertainty on the yield).

- The nominal background shape was kept, but scaled down by a factor of (1the statistical uncertainty on the yield).

- The upper background shape variation, described in Section 8.3.3 and normalized to the nominal yield, was used.

- The lower background shape variation, described in Section 8.3.3 and normalized to the nominal yield, was used.

For each bin of each observable, the deviation from the nominal value due to each systematic variation was determined; the results are shown in Figure 8.8. It can be seen that, in general, the effect of the shape variation is very small, on the order of about $2 \%$ or smaller for the majority of bins; the statistical uncertainty on the unfolded distribution (illustrated by the shaded bands) is the dominant source of uncertainty.

\subsubsection{Effect of the number of iterations}

As Figure 8.7 shows, the Bayesian unfolding algorithm tends to converge fairly quickly for most observables, with little variation in the unfolded result after the first few iterations. In order to quantify the effect of the choice of the particular number of iterations for each observable, the variation between the nominal result and those obtained with one more or one fewer iteration than the baseline number 


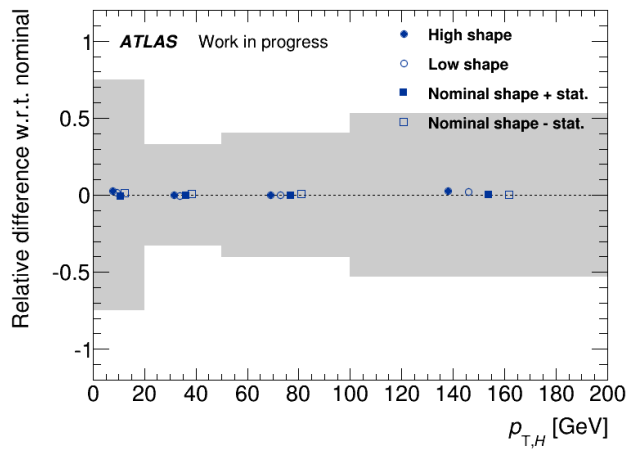

(a) $p_{\mathrm{T}, H}$

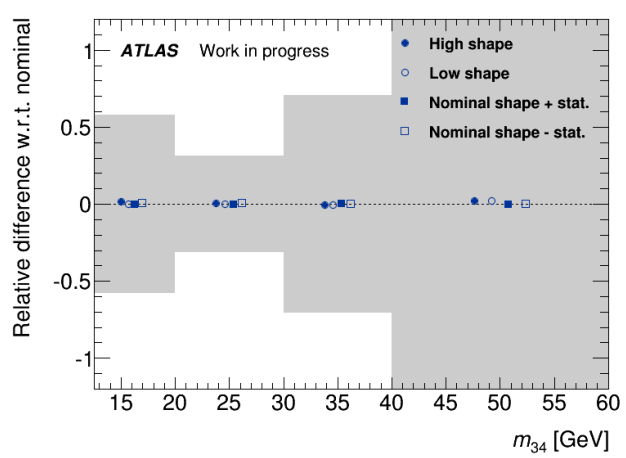

(c) $m_{34}$

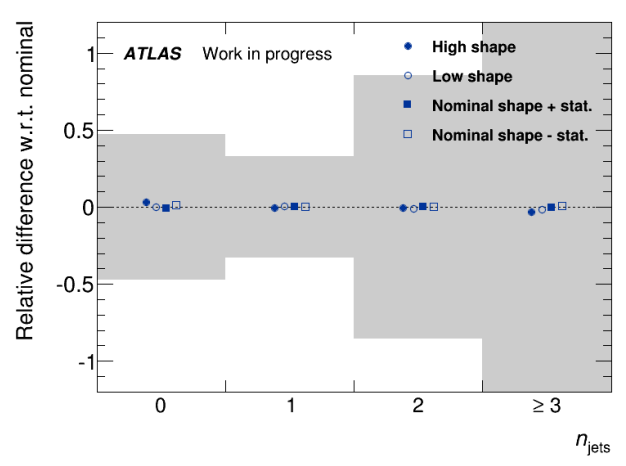

(e) $n_{\text {jets }}$

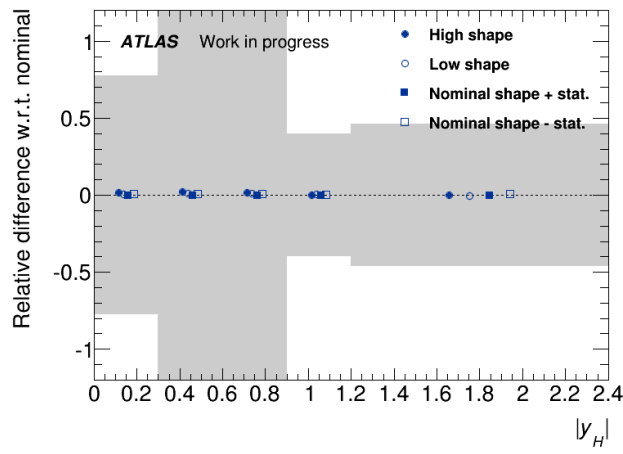

(b) $\left|y_{H}\right|$

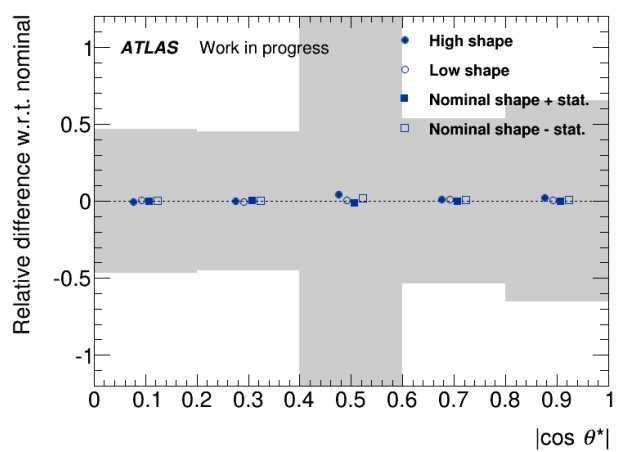

(d) $\left|\cos \theta^{*}\right|$

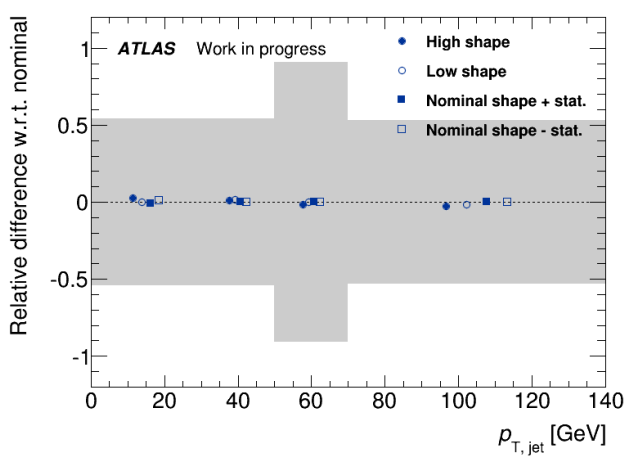

(f) $p_{\mathrm{T}, \text { jet }}$

Figure 8.8: Deviation from the nominal result due to varying the reducible background shapes. The points are slightly offset for clarity. The results are expressed in terms of the relative difference with respect to the results of the Bayesian unfolding method. Also shown for comparison are the statistical uncertainties on the nominal results (shaded bands). 
8. Measurement of the inclusive and differential fiducial cross-sections in the $H \rightarrow Z Z^{(*)} \rightarrow 4 \ell$ channel

of iterations chosen as described in Section 8.5.2 was determined. For $p_{\mathrm{T}, H}, m_{34}$, $\left|y_{H}\right|,\left|\cos \theta^{*}\right|$, and $n_{\text {jets }}$, the variation was on the order of $0.1 \%$ or less, and for $p_{\mathrm{T}}$, jet, the variation was approximately $0.1 \%-0.7 \%$. This variation was considered to be negligible.

\subsection{Conclusion}

The first measurements of the inclusive and differential fiducial cross-sections of the Higgs boson in the $H \rightarrow Z Z^{(*)} \rightarrow 4 \ell$ decay channel have been performed. For the differential measurements, six observables sensitive to kinematic properties of the Higgs boson and other Standard Model phenomena were chosen: $p_{\mathrm{T}, H},\left|y_{H}\right|$, $m_{34},\left|\cos \theta^{*}\right|, n_{\text {jets }}$, and $p_{\mathrm{T}, \text { jet }}$. The measured signal yield in the fiducial region is unfolded to the true, Born-level yield using bin-by-bin correction factors derived from simulation and corresponding to the inverse of the fiducial efficiency, i.e. the ratio of the number of events generated in the fiducial region to the number of events reconstructed in the fiducial region. The results have been compared to the Standard Model predictions, and no significant deviations from the expected values have been found.

An alternative unfolding method, known as the Bayesian iterative unfolding method and implemented in the RoOUnFOLD analysis framework, has been investigated. This method takes into account the effect of bin migration; i.e. that an event generated in a particular observable bin may be measured in another bin. This effect was assumed to be negligible in the publication [7] since the relatively low statistics of the $8 \mathrm{TeV}$ dataset necessitated the use of only a few large bins for each observable (this was, indeed, the case). The Bayesian method uses Bayes' theorem to compute the probability that a particular cause is responsible for a 
8. Measurement of the inclusive and differential fiducial cross-sections in the $H \rightarrow Z Z^{(*)} \rightarrow 4 \ell$ channel

given observed effect, taking into account the probability of bin migration. Given a simulated prior distribution for the observable and a response matrix describing the bin migration, the posterior probability can be calculated and an unfolded true distribution estimated; this estimate is then used to replace the prior distribution and the procedure is repeated for several iterations until a result is converged upon.

The differential cross-sections were determined for the six observables of interest using the Bayesian method, and compared to the results of the method of correction factors. It was found that the iterative procedure quickly converged, requiring between four and nine iterations, depending on the observable. The difference between the results of the Bayesian method and those obtained using the method of correction factors was found to be much smaller than the statistical uncertainty on the latter method; the statistical uncertainty was by far the dominant source of uncertainty for this measurement.

Given the speed with which the Bayesian unfolding algorithm converges on a result, despite the low signal yield in the $8 \mathrm{TeV}$ data, and given that the results agree well with the results of the nominal method, the outlook for the Bayesian method seems promising. Although the effect of bin migration in the $8 \mathrm{TeV}$ analysis was insignificant due to the low statistics and wide binning, this effect could become more significant for future analyses involving larger datasets in Run II, when a finer binning would be desired to perform measurements with a higher degree of precision. 


\section{Summary and outlook}

The path is long and difficult; there will be many challenges and dark moments, which need to be addressed with courage and determination. But the satisfaction of contributing to advance the limits of knowledge is extremely rewarding.

— Fabiola Gianotti

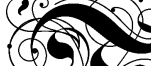

S ROM the initial start-up in 2008 of CERN's Large Hadron Collider, the Plargest and most energetic particle accelerator ever built, to the discovery of the Higgs boson in 2012, which provided the last missing piece of the Standard Model of particle physics and earned a Nobel Prize for the theorists who first proposed its existence, to the first precision measurements of the Higgs boson's properties, our understanding of the Universe is growing at a thrilling pace.

In order to fully investigate the Higgs boson and accept or refute the hypothesis that it conforms to Standard Model predictions, it is important to study all of its production modes and decay channels. One of these decay channels is the decay of the Higgs boson to four leptons, $H \rightarrow Z Z^{(*)} \rightarrow 4 \ell$ (where $\ell=e, \mu$ ), which has been studied using approximately $25 \mathrm{fb}^{-1}$ of data collected between 2011 and 2012 at $\sqrt{s}=7 \mathrm{TeV}$ and $8 \mathrm{TeV}$ with the ATLAS detector at the LHC. 
A wide variety of first measurements of its properties have been performed and published in peer-reviewed journals $[4,6,7,117]$. Using the data collected in the four-lepton decay channel, its mass has been measured to be:

$$
m_{H}=124.51 \pm 0.52 \text { (stat.) } \pm 0.06 \text { (syst.) GeV. }
$$

The signal strength in this channel, defined as the ratio of the observed signal to the Standard Model expectation, has been measured to be:

$$
\mu=1.66_{-0.34}^{+0.39} \text { (stat.) }{ }_{-0.14}^{+0.21} \text { (syst.). }
$$

The combination of the $H \rightarrow Z Z^{(*)} \rightarrow 4 \ell$ mass measurement with that of the $H \rightarrow \gamma \gamma$ channel yield the final combined ATLAS result:

$$
m_{H}=125.36 \pm 0.37 \text { (stat.) } \pm 0.18 \text { (syst.) GeV. }
$$

Its spin and parity are consistent with the Standard Model hypothesis of $J^{P}=0^{+}$, and the categorization of the candidate events, based on signatures associated with the dominant production modes at the LHC, yields no significant deviations from the Standard Model expectations. At this point, there is no experimental evidence to suggest that the Higgs boson is anything other than the Standard Model Higgs boson.

The four-lepton decay channel is often described as the "golden channel" due to its clean signal, which permits complete reconstruction of the Higgs boson's kinematic properties. Although the backgrounds in this channel are relatively small, the branching ratio is also small. Thus, every reconstructed event - and hence, every reconstructed lepton - counts in order to maximize the signal yield. 
In order to do so, highly efficient offline identification of electrons is necessary. In order to accomplish this goal, a cut-based algorithm, known as the MultiLePTON menu [3], was developed. The MultiLepton menu relied on inner detector track quality and calorimeter shower shape information in order to distinguish electrons from the mostly hadronic background. It performed very well in the role for which it was designed, with an identification efficiency of $\sim 95 \%$, while also rejecting twice as many background objects as the algorithm that was used previously. The MultiLepton menu was implemented in 2012 and contributed to the discovery of the Higgs boson.

Once the discovery of the Higgs boson had been accomplished, it became necessary to better understand the background in the four-lepton decay channel in order to ensure the selection of a clean sample of events for the purposes of precision measurements of the Higgs boson's properties. Since the total background in this channel is small, and since the dominant source of background is not well-modelled, it is important to be sure that it can be estimated reliably. For this reason, a datadriven method, known as the Transfer Factor method, was developed to estimate the contribution of the reducible electron-like background due to the $Z+$ jets, $Z+b \bar{b}$, and $t \bar{t}$ processes. This method was validated and found to be in good agreement with background estimates derived using alternate methods, and was implemented for the analyses of both the $7 \mathrm{TeV}$ and $8 \mathrm{TeV}$ data.

The first measurements of the inclusive and differential fiducial cross-sections have been performed with $20.3 \mathrm{fb}^{-1}$ of data collected at $\sqrt{s}=8 \mathrm{TeV}$. The inclusive fiducial cross-section was measured to be:

$$
\sigma_{\text {fid }}=2.11_{-0.47}^{+0.53} \text { (stat.) } \pm 0.8 \text { (syst.) fb, }
$$


in agreement with the Standard Model prediction of $1.30 \pm 0.13 \mathrm{fb}$. The differential cross-sections have been measured, using the method of bin-by-bin correction factors, for six observables sensitive to a wide range of Standard Model phenomena; no significant deviations from theoretical predictions are observed. An alternative technique for estimating the true observable distributions from the measured signal, known as the Bayesian iterative unfolding method, takes into account the possibility that an event generated in one particular observable bin may be measured in another due to resolution effects. This possibility is neglected in the published result [7], which is based on a small number of events distributed in bins wider than the resolution. The Bayesian iterative unfolding method has been investigated and has been found to yield results consistent with the nominal results obtained using the method of bin-by-bin correction factors.

The LHC is currently shut down for scheduled maintenance and upgrades, and is expected to resume operations in 2015, with the aim of collecting approximately $100 \mathrm{fb}^{-1}$ of data at centre-of-mass energies of $\sqrt{s}=13 \mathrm{TeV}$ and $14 \mathrm{TeV}$. With this larger dataset, future differential fiducial cross-section measurements will be performed using a finer binning, and so it is important to understand the role of bin migration and develop methods that take this effect into consideration. With this in mind, the Bayesian method shows particular promise and merits further study and possible implementation during Run II.

It would be outside the scope of this thesis to describe the entire analysis of the Higgs boson in the four-lepton decay channel. However, the work presented here has contributed in small, but important, ways and is a representation of the evolution of the analysis, from with the improved electron identification for the discovery, to the new method of estimating the reducible electron-like background in preparation for the first precision measurements, to the contribution to the first 
measurement of the differential fiducial cross-section. Finally, the investigation of the Bayesian unfolding method looks toward the future and the next round of precision measurements, as the LHC prepares to take data once again as we continue to study the Higgs boson in the context of the Standard Model and beyond. 


\section{A. Personal contributions to ATLAS}

The following is an approximate timeline of the author's personal contributions to the ATLAS experiment.

- Liquid argon calorimeter / forward detector shifter (2010 - 2011) Monitored the liquid argon calorimeters' and forward detectors' operational performance during data-taking in the ATLAS control room.

- Liquid argon calorimeter electronic calibration (2011) Monitored and validated daily and weekly calibration runs.

- Calo supershifter (2011 - 2012) Served as an on-call expert, responsible for training and supervising new shifters. Helped to facilitate the merging of the tile and liquid argon calorimeter shift crews.

- MultiLepton menu (2011 - 2012) Developed the MultiLepton menu for offline electron identification described in Chapter 4 for the $H \rightarrow Z Z^{(*)} \rightarrow$ $4 \ell$ search and discovery.

- Reducible electron-like background estimation (2012 - 2014) Developed the data-driven Transfer Factor method for estimating the reducible electron-like background in the $H \rightarrow Z Z^{(*)} \rightarrow 4 \ell$ channel, as decribed in Chapter 6 . 
- Differential fiducial cross-section measurement (2014) Studied the model-dependence of the method of correction factors, prepared background control plots, and investigated the use of the Bayesian iterative unfolding method; refer to Chapter 8. 


\section{B. Selected presentations}

The following presentations were given at major national or international workshops and conferences.

- GSF electron classification for $H \rightarrow Z Z^{(*)} \rightarrow 4 \ell$. ATLAS Canada Workshop, TRIUMF, Vancouver, BC, December 2011. Presented preliminary studies of bremsstrahlung-dependent variables to be used in the MultiLePTON offline electron identification menu described in Chapter 4.

- A study of reducible electron-like backgrounds in the search for the Higgs boson in the four-lepton decay channel at the ATLAS experiment. OttawaCarleton Institute for Physics seminar, Carleton University, Ottawa, ON, April 2013. Presented preliminary studies of the Transfer Factor method of background estimation described in Chapter 6 .

- Measurement of properties of the Higgs boson in bosonic decay channels using the ATLAS detector (on behalf of the ATLAS Collaboration). Phenomenology 2014 Symposium, University of Pittsburgh, Pittsburgh, PA, May 2014. Presented up-to-date results of precision measurements of the Higgs boson's properties from the $H \rightarrow Z Z^{(*)} \rightarrow 4 \ell, H \rightarrow \gamma \gamma$, and $H \rightarrow W^{+} W^{-}$channels. 


\section{Monte Carlo samples}

The following lists give the signal and background Monte Carlo samples used in this thesis, including the event generator used to produce the samples, the unique MCID code which serves to identify each particular sample, and the number of simulated events for each process.

\section{C.1 Signal samples}

\section{C.1.1 7 TeV samples}

\begin{tabular}{lllll}
\hline \hline Process & Generator & MCID & Events $\left(\times 10^{5}\right)$ & Comments \\
\hline \hline ggF & POWHEG/PYTHIA8.1 & 167892 & 800 & No final-state $\tau$-leptons \\
\hline VBF & POWHEG/PYTHIA8.1 & 167992 & 144 & No final-state $\tau$-leptons \\
\hline WH & POWHEG/PYTHIA8.1 & 160255 & 100 & \\
\hline ZH & POWHEG/PYTHIA8.1 & 160305 & 0.85 & \\
\hline$t \bar{t} \mathrm{H}$ & POWHEG/PYTHIA8.1 & 167562 & 100 & \\
\hline \hline
\end{tabular}

TABle C.1: List of signal Monte Carlo samples used in the $7 \mathrm{TeV} H \rightarrow Z Z^{(*)} \rightarrow$ $4 \ell$ analysis.

Table C.1 lists the $7 \mathrm{TeV}$ signal samples used in this thesis. All samples correspond to a Standard Model Higgs boson with $m_{H}=125 \mathrm{GeV}$ decaying via 
$H \rightarrow Z Z^{(*)} \rightarrow 2 \ell 2 \ell^{\prime}$, where $\ell, \ell^{\prime}=e, \mu, \tau$, unless otherwise specified. All samples were produced with the P1486 version tag and the MC11D configuration as described in Section 3.4.

\section{C.1.2 $8 \mathrm{TeV}$ samples}

\begin{tabular}{|c|c|c|c|c|}
\hline Process & Generator & MCID & Events $\left(\times 10^{5}\right)$ & Comments \\
\hline \multirow[t]{2}{*}{ ggF } & POWHEG/PYTHIA8.1 & 167892 & 800 & No final-state $\tau$-leptons; used for mass modelling (Chapter 7) \\
\hline & & 160155 & 200 & Used for cross-section measurement (Chapter 8) \\
\hline \multirow[t]{2}{*}{ VBF } & POWHEG/PYTHIA 8.1 & 167992 & 140 & No final-state $\tau$-leptons; used for mass modelling (Chapter 7) \\
\hline & & 160205 & 200 & Used for cross-section measurement (Chapter 8) \\
\hline WH & POWHEG/PYTHIA8.1 & 160255 & 100 & \\
\hline $\mathrm{ZH}$ & POWHEG/PYTHIA8.1 & 160305 & 0.75 & \\
\hline$t \bar{t} \mathrm{H}$ & POWHEG/PYTHIA8.1 & 167562 & 100 & \\
\hline \multirow[t]{3}{*}{ Spin-CP } & JHU/PYTHIA8.1 & 167120 & 100 & $J^{P}=0^{+}$; used for correction factor studies (Chapter 8) \\
\hline & & 167121 & 100 & $J^{P}=0^{-}$; used for correction factor studies (Chapter 8) \\
\hline & & 167122 & 100 & $J^{P}=2^{+} ;$used for correction factor studies (Chapter 8 ) \\
\hline
\end{tabular}

TABLE C.2: List of signal Monte Carlo samples used in the $8 \mathrm{TeV} H \rightarrow Z Z^{(*)} \rightarrow$ $4 \ell$ analysis.

Table C.2 lists the $8 \mathrm{TeV}$ signal samples used in this thesis. All samples correspond to a Standard Model Higgs boson with $m_{H}=125 \mathrm{GeV}$ decaying via $H \rightarrow Z Z^{(*)} \rightarrow 2 \ell 2 \ell^{\prime}$, where $\ell, \ell^{\prime}=e, \mu, \tau$, unless otherwise specified. All samples were produced with the $\mathrm{P} 1344$ version tag and the MC12C configuration as described in Section 3.4, with the exception of the spin-CP samples, which were produced with the MC12A configuration.

\section{C.2 Background samples}

\section{C.2.1 7 TeV samples}

Table C.3 lists the $7 \mathrm{TeV}$ reducible background samples used in this thesis. All samples were produced with the P1486 version tag and the MC11C configuration. 


\begin{tabular}{lllll}
\hline \hline Process & Generator & MCID & Events $\left(\times 10^{6}\right)$ & Comments \\
\hline \hline$Z+$ jets & AlPGEn/JimmY & $107650-107655$ & 10.7 & $Z(\rightarrow e e)+$ light jets \\
& & $116250-116255$ & 2.5 & $Z(\rightarrow e e)+$ light jets $\left(10<m_{e e}<40 \mathrm{GeV}\right)$ \\
& & $107660-107665$ & 10.7 & $Z(\rightarrow \mu \mu)+$ light jets \\
& & $116260-116265$ & 2.5 & $Z(\rightarrow \mu \mu)+$ light jets $\left(10<m_{\mu \mu}<40 \mathrm{GeV}\right)$ \\
& & $107670-107675$ & 15.6 & $Z(\rightarrow \tau \tau)+$ light jets \\
\hline$Z+b \bar{b}$ & AlPGEn/PyThiA8.1 & $181420-181422,181430-181432$ & 2.6 & $Z(\rightarrow e e)+b \bar{b}$ \\
& & $181425-181427,181435-181437$ & 2.6 & $Z(\rightarrow \mu \mu)+b \bar{b}$ \\
\hline$W Z$ & PythiA8.1 & 128971 & 0.05 & \\
\hline$t \bar{t}$ & Powheg/PythiA8.1 & 117050 & 1.0 & \\
\hline \hline
\end{tabular}

TABle C.3: List of $7 \mathrm{TeV}$ reducible background Monte Carlo samples used in this thesis.

Table C.4 lists the $7 \mathrm{TeV}$ irreducible background samples used in this thesis. All samples were produced with the P1486 version tag and the MC11D configuration.

\begin{tabular}{lllll}
\hline \hline Process & Generator & MCID & Events $\left(\times 10^{6}\right)$ & Comments \\
\hline \hline$Z Z^{(*)}$ & POWHEG/PYTHIA8.1 & $126937-126942$ & 4.5 & $q \bar{q} \rightarrow Z Z^{(*)} \rightarrow 2 \ell 2 \ell^{\prime}$, where $\ell, \ell^{\prime}=e, \mu, \tau$ \\
& & $167162-167167$ & 9.2 & $q \bar{q} \rightarrow Z Z^{(*)} \rightarrow 2 \ell 2 \ell^{\prime}$, where $\ell, \ell^{\prime}=e, \mu, \tau ;\left(100<m_{4 \ell}<150 \mathrm{GeV}\right)$ \\
& & $169690-169692$ & 0.2 & $q \bar{q} \rightarrow Z Z^{(*)} \rightarrow 2 \ell 2 \ell^{\prime}$, where $\ell, \ell^{\prime}=e, \mu ;\left(500<m_{4 \ell}<50000 \mathrm{GeV}\right)$ \\
& SHERPA & 161988 & 0.5 & $q \bar{q} \rightarrow Z Z^{(*)} \rightarrow 2 \ell 2 \ell^{\prime}$, where $\ell, \ell^{\prime}=e, \mu, \tau$ \\
& GG2ZZ & $116601-116603$ & 0.065 & $g g \rightarrow Z Z^{(*)} \rightarrow 2 \ell 2 \ell^{\prime}$, where $\ell, \ell^{\prime}=e, \mu$ \\
\hline \hline
\end{tabular}

TABle C.4: List of $7 \mathrm{TeV}$ irreducible background Monte Carlo samples used in this thesis.

\section{C.2.2 $8 \mathrm{TeV}$ samples}

\begin{tabular}{lllll}
\hline \hline Process & Generator & MCID & Events $\left(\times 10^{6}\right)$ & Comments \\
\hline \hline$Z+$ jets & AlPGEN/PythiA8.1 & $117650-117655,147105-147110$ & 27.7 & $Z(\rightarrow e e)+$ light jets \\
& & $178354-178358,178369-178373$ & 17.7 & $Z(\rightarrow e e)+$ light jets $\left(10<m_{e e}<60 \mathrm{GeV}\right)$ \\
& & $117660-117665,147113-147118$ & 27.7 & $Z(\rightarrow \mu \mu)+$ light jets \\
& & $178374-178378,178359-178363$ & 17.8 & $Z(\rightarrow \mu \mu)+$ light jets $\left(10<m_{\mu \mu}<60 \mathrm{GeV}\right)$ \\
& & $117670-117675,147121-147126$ & 44 & $Z(\rightarrow \tau \tau)+$ light jets \\
& & $178364-178368,178379-178383$ & 7.9 & $Z(\rightarrow \tau \tau)+$ light jets $\left(10<m_{\tau \tau}<60 \mathrm{GeV}\right)$ \\
\hline$Z+b \bar{b}$ & ALPGEN/PYTHIA8.1 & $181420-181422,181430-181432$ & 5.4 & $Z(\rightarrow e e)+b \bar{b}$ \\
& & $181425-181427,181435-181437$ & 5.4 & $Z(\rightarrow \mu \mu)+b \bar{b}$ \\
\hline$W Z$ & SHERPA & 147194,147197 & 6.2 & \\
\hline$t \bar{t}$ & Powheg/Pythia8.1 & 181078 & 40 & \\
\hline \hline
\end{tabular}

TABle C.5: List of $8 \mathrm{TeV}$ reducible background Monte Carlo samples used in this thesis. 
Table C.5 lists the $8 \mathrm{TeV}$ reducible background samples used in this thesis. All samples were produced with the P1344 version tag and the MC12B configuration.

\begin{tabular}{lllll}
\hline \hline Process & Generator & MCID & Events $\left(\times 10^{6}\right)$ & Comments \\
\hline \hline$Z Z^{(*)}$ & Powheg/PyTHIA8.1 & $126937-42$ & 5 & $q \bar{q} \rightarrow Z Z^{(*)} \rightarrow 2 \ell 2 \ell^{\prime}$, where $\ell, \ell^{\prime}=e, \mu, \tau$ \\
& & $167162-7$ & 8.7 & $q \bar{q} \rightarrow Z Z^{(*)} \rightarrow 2 \ell 2 \ell^{\prime}$, where $\ell, \ell^{\prime}=e, \mu, \tau ;\left(100<m_{4 \ell}<150 \mathrm{GeV}\right)$ \\
& $169690-2$ & 0.4 & $q \bar{q} \rightarrow Z Z^{(*)} \rightarrow 2 \ell 2 \ell^{\prime}$, where $\ell, \ell^{\prime}=e, \mu ;\left(500<m_{4 \ell}<50000 \mathrm{GeV}\right)$ \\
& SHERPA & 161988 & 0.5 & $q \bar{q} \rightarrow Z Z^{(*)} \rightarrow 2 \ell 2 \ell^{\prime}$, where $\ell, \ell^{\prime}=e, \mu, \tau$ \\
& GG2ZZ & $116601-3$ & 0.3 & $g g \rightarrow Z Z^{(*)} \rightarrow 2 \ell 2 \ell^{\prime}$, where $\ell, \ell^{\prime}=e, \mu$ \\
\hline \hline
\end{tabular}

TABle C.6: List of $8 \mathrm{TeV}$ irreducible background Monte Carlo samples used in this thesis.

Table C.6 lists the irreducible background samples used in this thesis. All samples were produced with the P1344 version tag and the MC12C configuration. 


\section{References}

[1] R. Munroe. xkcd, 2014. http://xkcd.com/896/.

[2] The ATLAS Collaboration. Observation of a new particle in the search for the Standard Model Higgs boson with the ATLAS detector at the LHC. Phys. Lett. B, 716, 2012. http://cds.cern.ch/record/1471031, arXiv: 1207.7214 [hep-ex].

[3] The ATLAS Collaboration. Electron efficiency measurements with the ATLAS detector using the 2012 LHC proton-proton collision data. ATLASCONF-2014-032, 2014. http://cds.cern.ch/record/1706245.

[4] The ATLAS Collaboration. Measurement of the Higgs boson mass from the $H \rightarrow \gamma \gamma$ and $H \rightarrow Z Z^{(*)} \rightarrow 4 \ell$ channels with the ATLAS detector using $25 \mathrm{fb}^{-1}$ of $p p$ collision data. Phys. Rev. D, 90:052004, 2014. http: //cds.cern.ch/record/1709081, arXiv: 1406 . 3827 [hep-ex].

[5] The ATLAS Collaboration. Measurements of the properties of the Higgs-like boson in the four lepton decay channel with the ATLAS detector using 25 $\mathrm{fb}^{-1}$ of proton-proton collision data. 48th Rencontres de Moriond on Electroweak Interactions and Unified Theories, ATLAS-CONF-2013-013, 2013. http://cds.cern. ch/record/1523699. 
[6] The ATLAS Collaboration. Measurements of Higgs boson production and couplings in the four-lepton channel in $p p$ collisions at center-of-mass energies of 7 and $8 \mathrm{TeV}$ with the ATLAS detector. Submitted to Phys. Rev. D, 2014. http://cds.cern.ch/record/1751040, arXiv:1408.5191 [hep-ex] .

[7] The ATLAS Collaboration. Fiducial and differential cross sections of Higgs boson production measured in the four-lepton decay channel in $p p$ collisions at $\sqrt{s}=8 \mathrm{TeV}$ with the ATLAS detector. Phys. Lett. B, 738:234-253, 2014. http://cds.cern.ch/record/1749689, arXiv: 1408.3226 [hep-ex] .

[8] F. Abe et al. (CDF Collaboration). Observation of top quark production in $p \bar{p}$ collisions with the Collider Detector at Fermilab. Phys. Rev. Lett., 74: 2626-2631, 1995. arXiv:hep-ex/9503002.

[9] S. Abachi et al. (DØ Collaboration). Search for high mass top quark production in $p \bar{p}$ collisions at $\sqrt{s}=1.8 \mathrm{TeV}$. Phys. Rev. Lett., 74:2422-2426, 1995. arXiv:hep-ex/9411001.

[10] K.A. Olive et al. (Particle Data Group). Chin. Phys. C, 38(090001), 2014. http://pdg.1bl.gov/.

[11] E. Noether. Invariante Variationsprobleme. Nachr. D. König. Gesellsch. D. Wiss. Zu Göttingen, Math-phys. Klasse, pages 235-257, 1918.

[12] D. J. Griffiths. Introduction to Elementary Particles. John Wiley \& Sons, Inc., 1987.

[13] M. E. Peskin and D. V. Schroeder. An introduction to quantum field theory. Addison-Wesley, 1995.

[14] A. Djouadi. The anatomy of electro-weak symmetry breaking. I: the Higgs boson in the Standard Model. 2005. arXiv:hep-ph/0503172. 
[15] S. L. Glashow. Partial-symmetries of Weak Interactions. Nuclear Physics, 22:579-588, 1961.

[16] S. Weinberg. A Model of Leptons. Physical Review Letters, 19:1264-1266, 1967.

[17] A. Salam. Weak and electromagnetic interactions. In N. Svartholm, editor, Elementary Particle Theory, page 367. Almquist and Wiksells, 1969.

[18] P. W. Higgs. Broken symmetries, massless particles and gauge fields. Phys. Lett., 12(2):132-133, 1964.

[19] P. W. Higgs. Broken Symmetries and the Masses of Gauge Bosons. Phys. Rev. Lett., 13(16):508-509, 1964.

[20] F. Englert and R. Brout. Broken Symmetry and the Mass of Gauge Vector Mesons. Phys. Rev. Lett., 13(9):321-323, 1964.

[21] G. S. Guralnik, C. R. Hagen, and T. W. B. Kibble. Global Conservation Laws and Massless Particles. Physical Review Letters, 13(20):585-587, 1964.

[22] R. Barate et al. Search for the Standard Model Higgs boson at LEP. Phys. Lett. B, 565:61-75, 2003. arXiv:hep-ex/0306033.

[23] Tevatron New Phenomena \& Higgs Working Group. Updated combination of CDF and DØ's searches for Standard Model Higgs boson production with up to $10.0 \mathrm{fb}^{-1}$ of data. 2012. arXiv:1207.0449 [hep-ex].

[24] M. Baak et al. Updated Status of the Global Electroweak Fit and Constraints on New Physics. Eur. Phys. J. C, 72(2003), 2012. arXiv:1107. $0975[$ hep-ph] . 
[25] R. P. Feynman. The behavior of hadron collisions at extreme energies. High Energy Collisions: Third International Conference at Stony Brook, N.Y., pages 237-249, 1969.

[26] LHC Higgs Cross-Section Working Group, 2014. https://twiki.cern.ch/ twiki/bin/view/LHCPhysics/LHCHXSWG.

[27] L. Evans and P. Bryant, editors. LHC machine. JINST, 3(S08001), 2008. http: //cds. cern. ch/record/1129806.

[28] P. Lefèvre and T. Pettersson, editors. The Large Hadron Collider: conceptual design. CERN-AC-95-05 LHC, 1995. http://cds.cern.ch/record/ 291782.

[29] CERN, 2014. http://home.web.cern.ch/.

[30] The ATLAS Collaboration. The ATLAS Experiment at the CERN Large Hadron Collider. JINST, 3(S08003), 2008. https://cdsweb.cern.ch/ record/1129811/.

[31] The CMS Collaboration. The CMS Experiment at the CERN LHC. JINST, 3(S08004), 2008. http://cds.cern.ch/record/1129810.

[32] The LHCb Collaboration. The LHCb detector at the LHC. JINST, 3 (S08005), 2008. https://cds.cern.ch/record/1129809/.

[33] The ALICE Collaboration. The ALICE Experiment at the CERN LHC. JINST, 3(S08002), 2008. http://cds.cern.ch/record/1129812.

[34] M. Bajko et al. Report of the task force on the incident of 19th September 2008 at the LHC. CERN-LHC-PROJECT-Report-1168, 2009. http://cds . cern.ch/record/1168025. 
[35] The ATLAS Collaboration. Improved luminosity determination in $p p$ collisions at $\sqrt{s}=7 \mathrm{TeV}$ using the ATLAS detector at the LHC. Eur. Phys. J. C, 73(2518), 2013. arXiv:1302.4393[hep-ex].

[36] ATLAS luminosity public results, 2013. https://twiki.cern.ch/twiki/ bin/view/AtlasPublic/LuminosityPublicResults.

[37] The ATLAS Collaboration. ATLAS Experiment - public results, 2014. https://twiki.cern.ch/twiki/bin/view/AtlasPublic/ EventDisplayStandAlone.

[38] The ATLAS Collaboration. ATLAS inner detector: technical design report, 1. CERN-LHCC-9\%-016, 1997. http://cds.cern.ch/record/331063.

[39] The ATLAS Collaboration. ATLAS inner detector: technical design report, 2. CERN-LHCC-97-017, 1997. http://cds.cern.ch/record/331064.

[40] The ATLAS Collaboration. ATLAS pixel detector: technical design report. CERN-LHCC-98-013, 1998. http://cds . cern.ch/record/381263.

[41] The ATLAS Collaboration. ATLAS pixel detector electronics and sensors. JINST, 3(P07007), 2008. http://cds.cern.ch/record/1119279.

[42] The ATLAS Collaboration. The barrel modules of the ATLAS semiconductor tracker. Nucl. Inst. Meth. A, 568, 2006. http://cds.cern.ch/record/ 974073.

[43] The ATLAS Collaboration. The ATLAS semiconductor tracker end-cap module. Nucl. Inst. Meth. A, 575, 2007. http://cds.cern.ch/record/ 1063618. 
[44] The ATLAS Collaboration. The silicon microstrip sensors of the ATLAS semiconductor tracker. Nucl. Inst. Meth. A, 578, 2007. http://cds.cern. ch/record/1019885.

[45] The ATLAS TRT Collaboration. The ATLAS TRT barrel detector. JINST, 3(P02014), 2008. http://cds.cern.ch/record/1094548.

[46] The ATLAS TRT Collaboration. The ATLAS TRT end-cap detectors. JINST, 3(P10003), 2008. http://cds.cern.ch/record/1151338.

[47] The ATLAS Collaboration. Electron reconstruction and identification efficiency measurements with the ATLAS detector using the 2011 LHC protonproton collision data. Eur. Phys. J. C, 74:2941, 2014. https://cds.cern. ch/record/1694142, arXiv: 1404.2240 [hep-ex].

[48] The ATLAS Collaboration. Liquid argon calorimeter technical design report. CERN-LHCC-96-041, 1996. http://cds.cern.ch/record/331061.

[49] The ATLAS Collaboration. Tile calorimeter technical design report. $C E R N$ LHCC-96-042, 1996. http://cds.cern.ch/record/331062.

[50] D. M. Gingrich et al. Construction, assembly and testing of the ATLAS hadronic end-cap calorimeter. JINST, 2(P05005), 2007. http://cds.cern . ch/record/1026994.

[51] A. Artamonov et al. The ATLAS forward calorimeter. JINST, 3(P02010), 2008. http://cds.cern.ch/record/1094547.

[52] The ATLAS Collaboration. Muon spectrometer technical design report. CERN-LHCC-97-022, 1997. http://cds.cern.ch/record/331068.

[53] ATLAS Level-1 Trigger Group. ATLAS level-1 trigger: technical design report. CERN-LHCC-98-014, 1998. http://cds.cern.ch/record/381429. 
[54] ATLAS HLT / DAQ / DCS group. ATLAS high-level trigger, data acquisition and controls: technical design report. CERN-LHCC-2003-022, 2003. http://cds. cern. ch/record/616089.

[55] ATLAS Experiment, 2014. http://www.atlas.ch/.

[56] The ATLAS Collaboration. Electron performance measurements with the ATLAS detector using the 2010 LHC proton-proton collision data. Eur. Phys. J. C, 72(1909), 2012. http://cds.cern.ch/record/1390775, arXiv: $1110.3174[$ hep-ex]

[57] W. Lampl et al. Calorimeter clustering algorithms: description and performance. ATL-LARG-PUB-2008-002, 2008. http://cds.cern.ch/record/ 1099735.

[58] The ATLAS Collaboration. Improved electron reconstruction in ATLAS using the Gaussian Sum Filter-based model for bremsstrahlung. ATLASCONF-2012-047, 2012. http://cds.cern.ch/record/1449796.

[59] R. E. Kalman. A new approach to linear filtering and prediction problems. Transactions of the ASME - Journal of Basic Engineering, 82 (Series D), 1960.

[60] R. Frühwirth. Track fitting with non-Gaussian noise. Comp. Phys. Comm., 100(1-2), 1997.

[61] H. Bethe and W. Heitler. On the stopping of fast particles and on the creation of positive electrons. Proc. R. Soc. A, 146, 1934.

[62] The ATLAS Collaboration. Expected performance of the ATLAS experiment: detector, trigger and physics. CERN-OPEN-2008-020, 2008. http://cds.cern.ch/record/1125884, arXiv:0901.0512 [hep-ex] . 
[63] The ATLAS Collaboration. Expected photon performance in the ATLAS experiment. ATL-PHYS-PUB-2011-007, 2011. http://cds.cern.ch/ record/1345329.

[64] The ATLAS Collaboration. Preliminary results on the muon reconstruction efficiency, momentum resolution, and momentum scale in ATLAS 2012 pp collision data. ATLAS-CONF-2013-088, 2013. http://cds.cern.ch/ record/1580207.

[65] The ATLAS Collaboration. Commissioning of the ATLAS muon spectrometer with cosmic rays. Eur. Phys. J. C, 70(875), 2010. http://cds.cern. ch/record/1275998, arXiv:1006.4384[physics.ins-det].

[66] M. Cacciari, G. P. Salam, and G. Soyez. The anti- $k_{t}$ jet clustering algorithm. JHEP, 04(063), 2008. arXiv:0802.1189[hep-ph].

[67] The ATLAS Collaboration. Performance of missing transverse momentum reconstruction in ATLAS studied in proton-proton collisions recorded in 2012 at $8 \mathrm{TeV}$. ATLAS-CONF-2013-082, 2013. http://cds.cern.ch/record/ 1570993.

[68] The ATLAS Collaboration. The ATLAS simulation infrastructure. Eur. Phys. J. C, 70:823-874, 2010. http://cds.cern.ch/record/1267853, arXiv: 1005 .4568[physics.ins-det].

[69] S. Agostinelli et al. Geant4 - a simulation toolkit. Nucl. Inst. Meth. Phys. Res. A, 506:250-303, 2003.

[70] J. Allison et al. Geant4 developments and applications. IEEE Trans. Nucl. Sci., 53:270-278, 2006. 
[71] The ATLAS Collaboration. ATLAS computing technical design report. CERN-LHCC-2005-022, 2005. http://cds. cern. ch/record/837738.

[72] The ATLAS Collaboration. Expected electron performance in the ATLAS experiment. ATL-PHYS-PUB-2011-006, 2011. http://cds.cern.ch/ record/1345327.

[73] S. Alioli et al. NLO Higgs boson production via gluon fusion matched with shower in POWHEG. JHEP, 04(002), 2009. arXiv:0812.0578[hep-ph].

[74] P. Nason and C. Oleari. NLO Higgs boson production via vector-boson fusion matched with shower in POWHEG. JHEP, 02(037), 2010. arXiv: $0911.5299[\mathrm{hep}-\mathrm{ph}]$.

[75] T. Sjostrand, S. Mrenna, and P. Z. Skands. PYTHIA 6.4 physics and manual. JHEP, 05(026), 2006. arXiv:hep-ph/0603175.

[76] T. Sjostrand, S. Mrenna, and P. Z. Skands. A brief introduction to PYTHIA 8.1. Comput. Phys. Commun., 178:852-867, 2008. arXiv: $0710.3820[$ hep-ph]

[77] P. Golonka and Z. Was. PHOTOS Monte Carlo: a precision tool for QED corrections in $Z$ and $W$ decays. Eur. Phys. J. C, 45, 2006. arXiv:hep-ph/ 0506026.

[78] LHC Higgs Cross Section Working Group, S. Dittmaier, C. Mariotti, G. Passarino, and R. Tanaka (Eds.). Handbook of LHC Higgs cross sections: 1. inclusive observables. CERN-2011-002, 2011. http://cds.cern.ch/record/ 1318996, arXiv:1101.0593[hep-ph].

[79] LHC Higgs Cross Section Working Group, S. Dittmaier, C. Mariotti, G. Passarino, and R. Tanaka (Eds.). Handbook of LHC Higgs cross sections: 
2. differential distributions. CERN-2012-002, 2012. http://cds.cern.ch/ record/1416519, arXiv:1201.3084[hep-ph].

[80] A. Djouadi, M. Spira, and P. M. Zerwas. Production of Higgs bosons in proton colliders: QCD corrections. Phys. Lett. B, 264, 1991.

[81] S. Dawson. Radiative corrections to Higgs boson production. Nucl. Phys. $B, 359,1991$.

[82] M. Spira et al. Higgs boson production at the LHC. Nucl. Phys. B., 453, 1995. arXiv:hep-ph/9504378.

[83] R. V. Harlander and W. B. Kilgore. Next-to-next-to-leading order Higgs production at hadron colliders. Phys. Rev. Lett., 88, 2002. arXiv:hep-ph/ 0201206.

[84] C. Anastasiou and K. Melnikov. Higgs boson production at hadron colliders in NNLO QCD. Nucl. Phys. B, 646:220-256, 2002. arXiv:hep-ph/0207004.

[85] V. Ravindran, J. Smith, and W. L. van Neerven. NNLO corrections to the total cross section for Higgs boson production in hadron hadron collisions. Nucl. Phys. B., 665:325-366, 2003. arXiv:hep-ph/0302135.

[86] S. Catani et al. Soft-gluon resummation for Higgs boson production at hadron colliders. JHEP, 07, 2003. arXiv: hep-ph/0306211.

[87] U. Aglietti et al. Two-loop light fermion contribution to Higgs production and decays. Phys. Lett. B, 595:432-441, 2004. arXiv: hep-ph/0404071.

[88] S. Actis et al. NLO electroweak corrections to Higgs boson production at hadron colliders. Phys. Lett. B., 670:12-17, 2008. arXiv:0809. 1301 [hep-ph]. 
[89] M. Ciccolini, A. Denner, and S. Dittmaier. Strong and electroweak corrections to the production of Higgs +2 jets via weak interactions at the LHC. Phys. Rev. Lett., 99:161803, 2007. arXiv:0707.0381 [hep-ph].

[90] M. Ciccolini, A. Denner, and S. Dittmaier. Electroweak and QCD corrections to Higgs production via vector-boson fusion at the LHC. Phys. Rev. D, 77: 013002, 2008. arXiv:0710.4749 [hep-ph].

[91] K. Arnold et al. VBFNLO: A parton level Monte Carlo for processes with electroweak bosons. Comput. Phys. Commun., 180:1661-1670, 2009. arXiv: 0811.4559 [hep-ph].

[92] T. Han and S. Willenbrock. QCD correction to the $p p \rightarrow W H$ and $Z H$ total cross-sections. Phys. Lett. B., 273:167-172, 1991.

[93] O. Brein, A. Djouadi, and R. Harlander. NNLO QCD corrections to the Higgs-strahlung processes at hadron colliders. Phys. Lett. B, 579:149-156, 2004. arXiv:hep-ph/0307206.

[94] M. L. Ciccolini, S. Dittmaier, and M. Kramer. Electroweak radiative corrections to associated $W H$ and $Z H$ production at hadron colliders. Phys. Rev. D., 68:073003, 2003. arXiv:hep-ph/0306234.

[95] Z. Kunszt. Associated production of heavy Higgs boson with top quarks. Nucl. Phys. B, 247:339, 1984.

[96] W. Beenakker et al. Higgs radiation off top quarks at the Tevatron and the LHC. Phys. Rev. Lett., 87:201805, 2001. arXiv:hep-ph/0107081.

[97] W. Beenakker et al. NLO QCD corrections to $t \bar{t} H$ production in hadron collisions. Nucl. Phys. B, 653:151-203, 2003. arXiv:hep-ph/0211352. 
[98] S. Dawson et al. Next-to-leading order QCD corrections to $p p \rightarrow t \bar{t} H$ at the CERN Large Hadron Collider. Phys. Rev. D, 67:071503, 2003.

[99] S. Dawson et al. Associated Higgs production with top quarks at the Large Hadron Collider: NLO QCD corrections. Phys. Rev. D, 68, 2003. arXiv: hep-ph/0305087.

[100] A. Djouadi, J. Kalinowski, and M. Spira. HDECAY: A program for Higgs boson decays in the standard model and its supersymmetric extension. Comput. Phys. Commun., 108:56-74, 1998. arXiv:hep-ph/9704448.

[101] A. Bredenstein et al. Precise predictions for the Higgs-boson decay $H \rightarrow$ $W W / Z Z \rightarrow 4$ leptons. Phys. Rev. D, 74, 2006. arXiv:hep-ph/0604011.

[102] A. Bredenstein et al. Radiative corrections to the semileptonic and hadronic Higgs-boson decays $H \rightarrow W W / Z Z \rightarrow 4$ fermions. JHEP, 02, 2007. arXiv: hep-ph/0611234.

[103] T. Melia et al. $W^{+} W^{-}, W Z$ and $Z Z$ production in the POWHEG BOX. JHEP, 1111, 2011. arXiv:1107.5051[hep-ph].

[104] T. Binoth, N. Kauer, and P. Mertsch. Gluon-induced QCD corrections to $p p \rightarrow Z Z \rightarrow \bar{\ell} \ell^{\prime} \overline{\ell^{\prime}} .2008$. arXiv:0807.0024[hep-ph].

[105] M. L. Mangano et al. ALPGEN, a generator for hard multiparton processes in hadronic collisions. JHEP, 07(001), 2003. arXiv: hep-ph/0206293.

[106] T. Gleisberg et al. Event generation with SHERPA 1.1. JHEP, 02(007), 2009. arXiv:0811.4622[hep-ph].

[107] The ATLAS Collaboration. A measurement of the ATLAS muon reconstruction and trigger efficiency using $J / \psi$ decays in p-p collisions at $\sqrt{s}=7 \mathrm{TeV}$. ATLAS-CONF-2011-021, 2011. http://cds . cern.ch/record/1336750. 
[108] The ATLAS Collaboration. Electron and photon energy calibration with the ATLAS detector using LHC Run 1 data. Eur. Phys. J. C, 74:3071, 2014. https://cds.cern.ch/record/1744017, arXiv:1407 .5063 [hep-ex].

[109] The ATLAS Collaboration. Measurement of the muon reconstruction performance of the ATLAS detector using 2011 and 2012 LHC proton-proton collision data. Eur. Phys. J. C, 74:3130, 2014. http://cds.cern.ch/record/ 1743068, arXiv:1407.3935[hep-ex].

[110] The ATLAS Collaboration. Jet energy scale and its systematic uncertainty in proton-proton collisions at $\sqrt{s}=7 \mathrm{TeV}$ with ATLAS 2011 data. (submitted to Eur. Phys. J. C), 2014. https://cds.cern.ch/record/1705576, arXiv: $1406.0076[$ hep-ex]

[111] P. Speckmayer et al. The toolkit for multivariate data analysis, TMVA 4. J. Phys.: Conf. Ser., 219(032057), 2010.

[112] E. Benhar Noccioli, 2013. Private communication.

[113] M. Botje et al. The PDF4LHC working group interim recommendations. 2011. arXiv:1101.0538[hep-ph].

[114] The ATLAS Collaboration. Measurement of Higgs boson production in the diphoton decay channel in $p p$ collisions at center-of-mass energies of 7 and $8 \mathrm{TeV}$ with the ATLAS detector. Phys. Rev. D, 90(112015), 2014. http: //cds.cern.ch/record/1752602, arXiv:1408.7084 [hep-ex].

[115] The CMS Collaboration. Measurement of the properties of a Higgs boson in the four-lepton final state. Phys. Rev. D, 89(092007), 2014. http://cds . cern.ch/record/1637951, arXiv:1312.5353 [hep-ex]. 
[116] The CMS Collaboration. Precise determination of the mass of the Higgs boson and tests of compatibility of its couplings with the standard model predictions using proton collisions at 7 and 8 tev. Submitted to Eur. Phys. J. C, 2014. http://cds.cern.ch/record/1979247, arXiv:1412.8662 [hep-ex].

[117] The ATLAS Collaboration. Evidence for the spin-0 nature of the Higgs boson using ATLAS data. Phys. Lett. B, 726:120-144, 2013. http://cds . cern.ch/record/1559925, arXiv:1307.1432 [hep-ex].

[118] S. Bolognesi et al. On the spin and parity of a single-produced resonance at the LHC. Phys. Rev. D, 86(095031), 2012. arXiv:1208.4018[hep-ph].

[119] The ATLAS Collaboration. Measurement of fiducial and differential cross sections for Higgs boson production in the diphoton channel at $\sqrt{s}=8 \mathrm{TeV}$ with the ATLAS detector. JHEP, 09(112), 2014. http://cds.cern.ch/ record/1743175, arXiv: 1407.4222 [hep-ex] .

[120] LHC Higgs Cross Section Working Group, S. Heinemeyer, C. Mariotti, G. Passarino, R. Tanaka (Eds.). Handbook of LHC Higgs cross sections: 3. Higgs properties. CERN-2013-004, 2013. https://cds.cern.ch/record/ 1559921, arXiv: 1307.1347 [hep-ph] .

[121] Y. Gao et al. Spin determination of single-produced resonances at hadron colliders. Phys. Rev. D, 81(075022), 2010. arXiv:1001.3396 [hep-ph].

[122] The ATLAS Collaboration. Jet energy resolution in proton-proton collisions at $\sqrt{s}=7 \mathrm{TeV}$ recorded in 2010 with the ATLAS detector. Eur. Phys. J. C, 73:2306, 2013. https://cds.cern.ch/record/1489592, arXiv:1210. $6210[$ hep-ex] . 
[123] The ATLAS Collaboration. Jet energy measurement with the ATLAS detector in proton-proton collisions at $\sqrt{s}=7 \mathrm{TeV}$. Eur. Phys. J. C, 73:2304, 2013. http://cds.cern.ch/record/1409965, arXiv:1112.6426 [hep-ex].

[124] K. Hamilton, P. Nason, and G. Zanderighi. MINLO: Multiscale Improved NLO. JHEP, 2012:155, 2012. arXiv:1206 .3572 [hep-ph].

[125] D. de Florian et al. Higgs boson production at the LHC: transverse momentum resummation effects in the $H \rightarrow 2 \gamma, H \rightarrow W W \rightarrow \ell \nu \ell \nu$ and $H \rightarrow Z Z \rightarrow 4 \ell$ decay modes. 2012. arXiv:1203.6321.

[126] M. Grazzini and H. Sargsyan. Heavy-quark mass effects in Higgs boson production at the LHC. 2013. arXiv:1306.4581.

[127] G. Cowan et al. Asymptotic formulae for likelihood-based tests of new physics. Eur. Phys. J. C, 71:1554, 2011. arXiv:1007.1727[physics. data-an].

[128] G. D'Agostini. A multidimensional unfolding method based on Bayes' theorem. Nucl. Inst. Meth. A, 362:487, 1995.

[129] T. Adye. RooUnfold: ROOT unfolding framework, 2012. http://hepunx. rl.ac.uk/ adye/software/unfold/RooUnfold.html.

[130] T. Adye. Unfolding algorithms and tests using RooUnfold. 2011. arXiv: 1105.1160 [physics.data-an].

[131] R. Brun and F. Rademakers. ROOT - an object oriented data analysis framework. Nucl. Inst. Meth. A, 389:81, 1997. http://root.cern.ch/.

[132] G. Cowan. Statistical Data Analysis. Oxford University Press, 1998. 